\title{
Gene analysis for studying the process of weight regain after weight loss
}

Citation for published version (APA):

Roumans, N. J. T. (2017). Gene analysis for studying the process of weight regain after weight loss.

[Doctoral Thesis, Maastricht University]. Gildeprint Drukkerijen. https://doi.org/10.26481/dis.20170623nr

Document status and date:

Published: 01/01/2017

DOI:

10.26481/dis.20170623nr

Document Version:

Publisher's PDF, also known as Version of record

\section{Please check the document version of this publication:}

- A submitted manuscript is the version of the article upon submission and before peer-review. There can be important differences between the submitted version and the official published version of record.

People interested in the research are advised to contact the author for the final version of the publication, or visit the DOI to the publisher's website.

- The final author version and the galley proof are versions of the publication after peer review.

- The final published version features the final layout of the paper including the volume, issue and page numbers.

Link to publication

\footnotetext{
General rights rights.

- You may freely distribute the URL identifying the publication in the public portal. please follow below link for the End User Agreement:

www.umlib.nl/taverne-license

Take down policy

If you believe that this document breaches copyright please contact us at:

repository@maastrichtuniversity.nl

providing details and we will investigate your claim.
}

Copyright and moral rights for the publications made accessible in the public portal are retained by the authors and/or other copyright owners and it is a condition of accessing publications that users recognise and abide by the legal requirements associated with these

- Users may download and print one copy of any publication from the public portal for the purpose of private study or research.

- You may not further distribute the material or use it for any profit-making activity or commercial gain

If the publication is distributed under the terms of Article $25 \mathrm{fa}$ of the Dutch Copyright Act, indicated by the "Taverne" license above, 
Gene analysis for studying the process of weight regain after weight loss 
The studies presented in this thesis were performed within NUTRIM School for Nutrition and Translational Research in Metabolism, which participates in the Graduate School VLAG (Food Technology, Agrobiotechnology, Nutrition and Health Sciences), accredited by the Royal Netherlands Academy of Arts and Sciences.
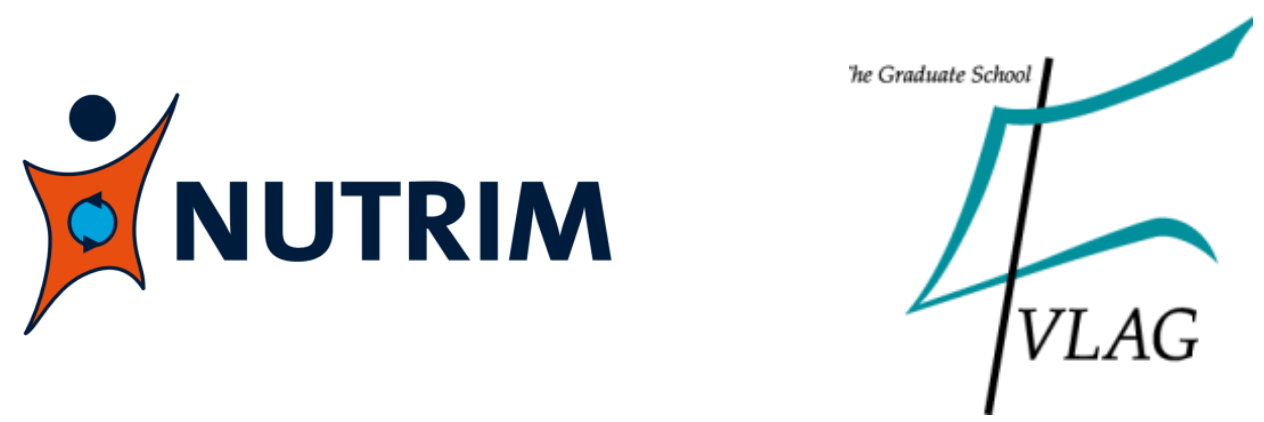

The studies described in this thesis were supported by a grant from Netherlands Organisation for Scientific Research (NWO) TOP, grant number: 200500001.

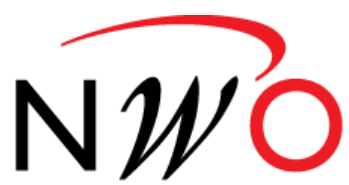

Netherlands Organisation for Scientific Research

Cover design: Nadia J.T. Roumans and Evelien Jagtman (http://evelienjagtman.com/) Layout: Nadia J.T. Roumans

Printed by: Gildeprint - www.gildeprint.nl

(C) Nadia J.T. Roumans, Maastricht 2016

For articles published or accepted for publication, the copyright has been transferred to the respective publisher. No parts of this thesis may be reproduced, stored in a retrieval system, or transmitted in any form or by any means without the permission of the author, or, when appropriate, from the publishers of the manuscript. 


\title{
Gene analysis for studying the process of weight regain after weight loss
}

\author{
PROEFSCHRIFT \\ ter verkrijging van de graad doctor aan de Universiteit Maastricht, \\ op gezag van de Rector Magnificus, Prof. dr. Rianne M. Letschert, \\ volgens het besluit van het College van Decanen, \\ in het openbaar te verdedigen \\ op vrijdag 23 Juni 2017 om 10.00 uur \\ door \\ Nadia Johanna Theodora Roumans
}

Geboren te Zeven in Duitsland op 3 Maart 1989 


\section{Promotoren}

Prof. dr. E.C.M. Mariman

Prof. dr. M.A. van Baak

\section{Beoordelingscommissie}

Prof. dr. E.E. Blaak, voorzitter

Prof. dr. I.C. Arts

Prof. dr. A.H. Kersten (Wageningen University \& Research)

Dr. E.M. van Schothorst (Wageningen University \& Research)

Prof. dr. L.P.A.J. Schrauwen

\section{Financiële ondersteuning}

Nederlandse Organisatie voor Wetenschappelijk Onderzoek (NWO): TOP grant 200500001 


\section{Content}

Chapter 1 General introduction

Chapter 2 Variation in extracellular matrix genes is associated with weight regain after weight loss in a sex-specific manner

Chapter 3 A role for leukocyte and ECM remodelling of adipose tissue in the risk for weight regain after weight loss

Chapter 4 Weight loss-induced stress in subcutaneous adipose tissue is related to weight regain

Chapter 5 Weight loss-induced cellular stress in subcutaneous adipose tissue and the risk for weight regain in overweight and obese adults

Chapter 6 Relation between stress- and ECM-related genes and their effect on weight regain

Chapter 7 General discussion

Addendum Summary 157

Samenvatting

Valorization 167

Dankwoord/Acknowledgements 173

Curriculum Vitae

List of Publications 



\section{Chapter 1}

General introduction 


\section{Obesity}

The prevalence of overweight and obesity has increased worldwide, presenting major health problems (1). In 2014, the World Health Organization estimated that of adults aged 18 and over, 39\% were overweight and $13 \%$ were obese (2). The prevalence of overweight and obesity is assessed by using body mass index (BMI), which is defined as weight in kilograms divided by the square of height in meters $\left(\mathrm{kg} / \mathrm{m}^{2}\right)$. Individuals with a BMI $\geq 25 \mathrm{~kg} / \mathrm{m}^{2}$ are classified as overweight and individuals with a $\mathrm{BMI} \geq 30 \mathrm{~kg} / \mathrm{m}^{2}$ are classified as obese. The increasing prevalence of obesity is a major health concern since it increases the risk for developing type 2 diabetes mellitus (3), cardiovascular diseases (4) and certain types of cancer (5). In fact, overweight- and obesity-related health problems were estimated to cause 3.4 million deaths in 2010 (1). Overweight and obesity occur when there is a disturbance in the energy balance, which is determined by energy intake and expenditure. When energy balance is maintained, energy intake and energy expenditure are equal (Figure 1). If energy intake exceeds energy expenditure, the excessive energy is stored as triglycerides (TG) in the white adipose tissue (WAT). This causes weight gain and eventually obesity. On the other hand, if energy expenditure exceeds energy intake, the stored TG are used as fuel for other energy demanding processes elsewhere in the body.

\section{Energy intake}

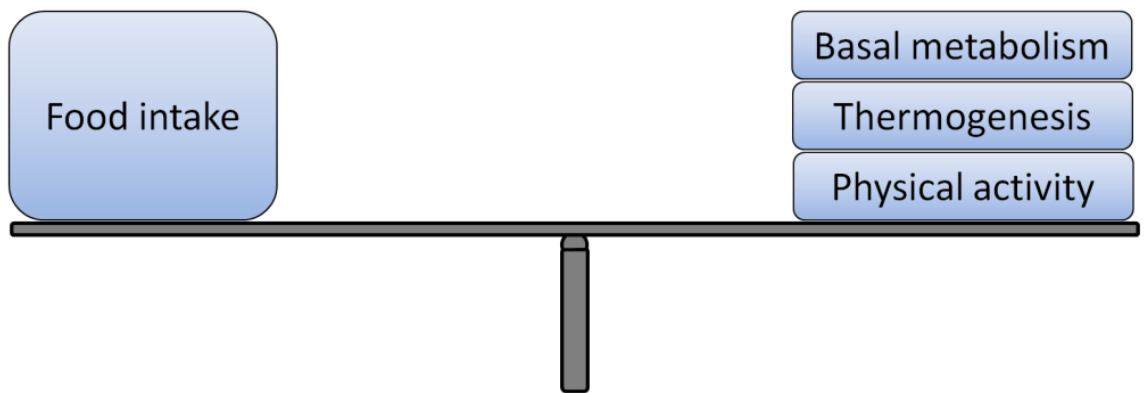

Figure 1: Balance between energy intake and energy expenditure.

Nowadays the often-observed energy imbalance is mainly caused by the combination of a high calorie intake and a low physical activity (6). In our modern society, food is abundantly available and is also energy dense whereas the demand of physical activity is strongly reduced. However, this 'obesogenic' environment cannot fully explain the development of obesity at an individual level, since many people seem to be protected against obesity. This raises the question which factors determine the individual susceptibility to become obese. There is evidence that an individual's susceptibility for gaining weight is determined in part by genetics $(7,8)$. Numerous genes have been identified that are associated with obesity (9). Genetic variants or single nucleotide polymorphisms (SNPs), like the polymorphism in the fat mass and obesity-associated (FTO) gene, have been linked to obesity (10-12). An individual with a genetic predisposition is unlikely to develop obesity without being exposed to an obesity-promoting environment, suggesting gene-environment interaction effects (13). For example, the body weight development of children is influenced by the BMI of their parents. A higher BMI for the parent is linked to a higher BMI in their children (14). This parental association is due to the combination of a shared genetic background and a shared lifestyle. Besides genetics, other factors also contribute to the individual's susceptibility to become obese, such as aging: when people become older they tend to gain weight (15). Also, social, cultural and economic status, and inadequate sleep are associated with the prevalence of obesity $(8,16,17)$. 


\section{Weight loss and weight regain}

There is a simple remedy to obesity, i.e. losing weight by limiting energy/food intake and increasing daily physical activity for a longer period of time. A body weight reduction of 5-10\% reduces disease risk and improves the metabolic profile resulting in positive health outcomes $(18,19)$. However, the greatest challenge is the seemingly inevitable weight regain after weight loss, the so-called "yoyoeffect". In general up to $80 \%$ of the people are unsuccessful in maintaining weight loss $(20,21)$ defined as "keeping off an intentional loss of at least $10 \%$ body weight for at least one year" (22). On average about $70 \%$ of the lost weight is regained within two years after the dietary intervention (23). Still, some individuals are able to keep off their lost weight, pointing to an individual susceptibility for weight maintenance or weight regain. Similarly to the predisposition to develop overweight or obesity, the individual susceptibility to successful weight loss and weight maintenance seems to be determined by multiple factors. Genetic, behavioural and physiological factors related to body weight might directly or indirectly affect weight loss and weight maintenance. Hormonal and genetic factors change in response to weight loss, thereby either changing to a pre-obese state or favouring weight regain. For instance, circulating mediators of appetite that enhance weight regain did not return to pre-weight loss levels one year after the start of the weight loss intervention (24). In addition, during weight loss circulating leptin concentrations decrease which could lead to a postweight loss starvation reaction resulting in reduced energy expenditure and increased food intake $(25,26)$. Restoring leptin to pre-weight loss circulating levels reverses the starvation reaction by decreasing energy intake (27) and by increasing resting- and activity-induced energy expenditure (28).

A more drastic method for losing weight is bariatric surgery, in which the size and/or the digestive capacity of the stomach are reduced. However, a relapse to increased calorie intake is not uncommon in the long term (29). There are some behaviour modifications that can help to prevent weight regain such as self-monitoring of body weight and food intake, having a physically active lifestyle and having a regular meal rhythm with reduced frequency of snacks (30).

\section{The role of the adipose tissue in obesity}

Adipose tissue is found in specific locations in the human body, which are referred to as adipose depots. The adipose depots are located beneath the skin (subcutaneous adipose tissue) and around the internal organs (visceral adipose tissue) (31). The main parenchymal cells of the adipose organ are called adipocytes. Adipocytes can be split into two types based on morphology and function: white adipocytes and brown adipocytes. White adipocytes are spherical cells containing one big lipid droplet that fills about $90 \%$ of their volume, whereas brown adipocytes are polygonal cells containing multiple lipid droplets and more mitochondria than white adipocytes (Figure 2) (31). White adipocytes store and release energy, while brown adipocytes burn energy for thermogenesis. The white adipocytes are activated by eating or starving, whereas activation of brown adipocytes can be regulated by cold exposure. In this thesis we focus on the subcutaneous WAT and white adipocytes. 


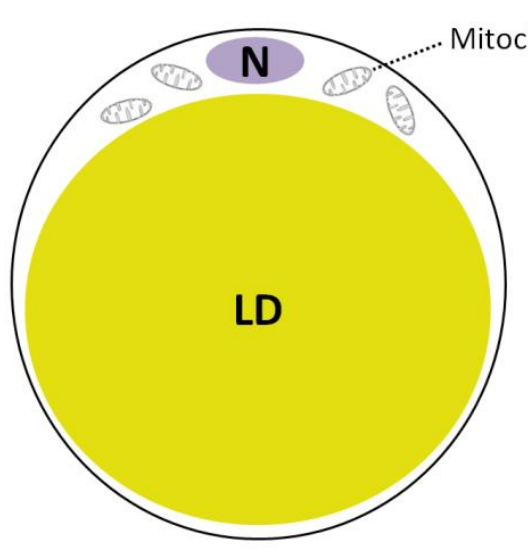

White adipocyte

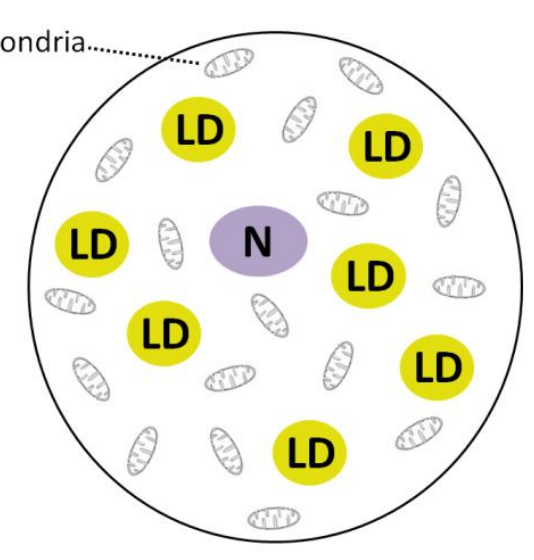

Brown adipocyte

Figure 2: Schematic depiction of white and brown adipocytes containing a nucleus (N), lipid droplets (LD) and mitochondria.

The WAT is a major energy-storing tissue. In a period of a negative energy balance adipocytes will break down TG and release fatty acids (FA) in the blood to fuel energy-demanding tissues. In a period of a positive energy balance adipocytes take up lipids that are converted into TG and stored in lipid droplets (32). Storage of TG leads to WAT expansion, which is determined by an increase in adipocyte size (hypertrophy) and/or number (hyperplasia) $(33,34)$. Metabolic disturbances associated with obesity, such as insulin resistance, are associated with adipocyte hypertrophy (35), underlining the important function of WAT in obesity. Besides its role in lipid metabolism, including TG storage and FA release, WAT also provides mechanical protection, insulation of the body and it can secrete adipokines which contribute to the regulation of biological functions such as appetite and satiety, insulin secretion and sensitivity, energy expenditure, and inflammation (36).

\section{The adipose tissue and weight regain}

As mentioned, long-term weight loss maintenance is difficult and $80 \%$ of the people are unsuccessful $(20,21)$. This provides a substantial challenge in obesity treatment. Long-term weight loss maintenance is dependent on energy intake. Therefore, hormones that regulate hunger and satiety are important with respect to the risk for weight regain. One of these hormones, leptin, decreases during weight loss, which leads to a higher risk for increased energy intake after weight loss (37). Injection of leptin during the weight maintenance period causes a change in activity of the brain regions responsible for energy intake regulation and as such decrease the risk for high energy intake (27). For the hormone ghrelin, produced by cells in the stomach, it has been reported that higher baseline levels seem to predict better weight maintenance after weight loss (38). However, changes in the hormone secretion profile do not exclusively contribute to the risk for weight regain. After weight loss, the gene expression profile is altered to enhance energy conservation and storage within the WAT (39-41). Markers of oxidative stress and inflammatory cytokines, which are also known to suppress appetite and increase energy expenditure, decline (4244). This improved metabolic regulation in the adipose tissue is likely due to the reduction in adipocyte size because smaller adipocytes are more insulin sensitive, have a lower rate of turnover of the stored lipid, and have higher expression of genes favouring the storage of energy (45-47). Thus, adaptations in adipocyte morphology may lead to the renewed storage of energy when nutrients once again become readily available after dieting and as such contribute to the drive to regain 
weight. Part of the research described in this thesis will focus on extending knowledge about the involvement of adipocyte morphology and composition on the risk for weight regain.

\section{Extracellular matrix in adipocyte cellular metabolism}

The structure and composition of the WAT are crucial for fulfilling its function in energy storage, energy metabolism, thermal insulation and mechanical protection. Each adipocyte is surrounded by a thick extracellular matrix (ECM) which is composed of numerous protein and carbohydrate components including collagens, fibronectin, laminins and proteoglycans (48). As mentioned, white adipocytes store TG in a single fat droplet almost entirely filling the cell. A lipid monolayer separates the stored fat from the cytosol and mechanical stress on this monolayer may easily lead to disruption of the fat droplet which is detrimental for the adipocyte. Thus, mechanical pressure on the outside of the cell should be avoided, which can be achieved by a strong ECM. The make-up of the ECM is important for its strength and the adipocyte maintains this by continuously renewing the components of the ECM. This constant renewal is mediated by enzymes promoting the construction of the ECM and by enzymes involved in its degradation. During energy excess and deficiency, the WAT must be able to quickly respond to the increase or decrease of the adipocyte volume by altering the structure, size, and shape of the adipose tissue, generally referred to as adipose tissue remodelling. In case of a positive energy balance, the size of individual adipocytes increases due to an increase in TG storage. This enlargement is limited since each adipocyte has a maximum volume that does not allow further expansion (49). Possibly, the maximum cell size is determined by the ability of the adipocyte to keep the extracellular matrix in such a condition that it can protect the cell against disruption (50). Adipocytes reaching the maximum cell size will trigger the formation of new adipocytes from a pre-adipocyte precursor population (51). During a negative energy balance, the release of fatty acids decreases the volume of adipocytes. When adipocytes have lost a large part of their fat content equilibrium may be reached, preventing further fatty acid release from adipocytes.

\section{Cellular stress in adipocyte cellular metabolism and weight regain}

The decrease in adipocyte volume because of the loss of TG during a negative energy balance requires adjustments of the ECM to fit the new adipocyte size. These adjustments are accomplished by the turnover of extracellular matrix proteins which is an energy-demanding process (50). It is hypothesized that during weight loss, such energy may not be available. As a consequence, the ECM remodelling cannot keep up with the decrease of adipocyte volume, thereby causing an improper fit between adipocyte and the surrounding ECM which leads to tension and cellular stress. As a reaction to this, the adipocyte wants to counteract the cellular stress. In this respect, the adipocyte could go into apoptosis. However, the average lifespan of an adipocyte is 10 years suggesting that apoptosis is not likely (52). Probably, a more efficient way to reduce the cellular stress is to re-store TG in the adipocyte at which the cell returns to the original volume. As a consequence the body weight of the host increases (Figure 3) (53). As soon as a person stops dieting and returns to energy balance, the capacity of the adipocytes to take up free fatty acid and glucose is increased (54). This suggests that cellular stress accumulated in adipocytes during a negative energy balance, due to the misfit between cell volume and ECM, is a driving force behind the risk for weight regain. The main objective of this thesis is to extend the knowledge about the involvement of adipocyte stress-and ECM-related factors in the weight regain response after weight loss. 


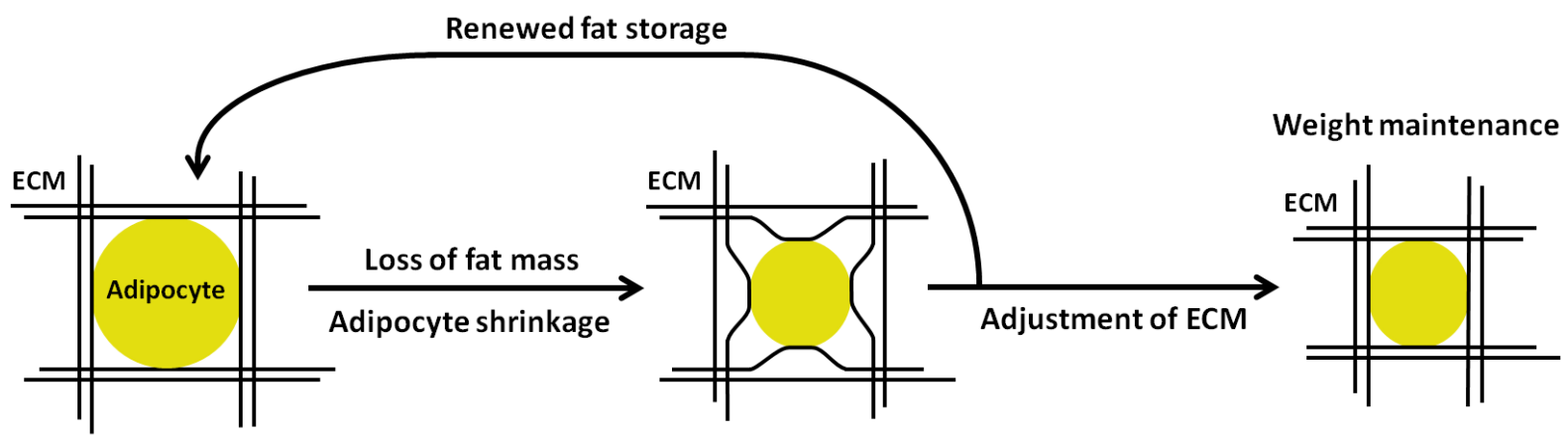

Figure 3: Cellular stress relief in the adipocyte by adjustment of the ECM or re-storage of fat.

\section{Outline of the thesis}

The first part of this thesis describes research on the relation between extracellular matrix and weight regain to find possible predictors of weight regain. Chapter $\mathbf{2}$ focuses on determining whether genetic variations in extracellular matrix related genes are associated with weight regain among participants of a Pan-European, randomized, controlled dietary intervention study called the DiOGenes study. In Chapter 3, the relation between weight regain and changes in expression of extracellular matrix genes was investigated. This was analysed in a randomized, controlled dietary intervention study (the so-called "yoyo-study") in which participants lost weight by either a 5-week very-low-calorie diet (VLCD) or a 12-week low-calorie diet (LCD), with a subsequent 4-week weight stable diet, and a 9-month follow-up period.

The second part of this thesis describes research on the relation between stress-related genes or proteins and weight regain in two dietary intervention studies. The first study consisted of an 8-week VLCD followed by a 10-months follow-up period. A comparison is made between participants regaining weight and participants maintaining weight with regard to levels of stress-related factors during weight loss and weight maintenance (Chapter 4 ). The second study used was the yoyo-study. In Chapter 5, the relation between weight regain and changes in the expression of genes for stress proteins during weight loss and weight stabilization was investigated to find possible predictors of weight regain. The relation between adipocyte stress- and ECM-related genes with regard to weight regain is reported in Chapter 6. Finally, the results from the described studies in this thesis are summarized and discussed in Chapter 7. 


\section{References}

1. Ng M, Fleming T, Robinson M, Thomson B, Graetz N, Margono C et al. Global, regional, and national prevalence of overweight and obesity in children and adults during 1980-2013: a systematic analysis for the Global Burden of Disease Study 2013. Lancet, 2014. 384(9945):766-81.

2. WHO. Obesity and overweight. Fact sheet №311, 2016.

3. Kahn SE, Hull RL, Utzschneider KM. Mechanisms linking obesity to insulin resistance and type 2 diabetes. Nature, 2006. 444(7121):840-6.

4. Van Gaal LF, Mertens IL, De Block CE. Mechanisms linking obesity with cardiovascular disease. Nature, 2006. 444(7121):875-80.

5. Calle EE, Rodriguez C, Walker-Thurmond K, Thun MJ. Overweight, obesity, and mortality from cancer in a prospectively studied cohort of U.S. adults. The New England journal of medicine, 2003. 348(17):1625-38.

6. Hill JO, Wyatt HR, Peters JC. Energy balance and obesity. Circulation, 2012. 126(1):126-32.

7. Maclean PS, Bergouignan A, Cornier MA, Jackman MR. Biology's response to dieting: the impetus for weight regain. Am J Physiol Regul Integr Comp Physiol, 2011. 301(3):R581-600.

8. Maes HH, Neale MC, Eaves $\amalg$. Genetic and environmental factors in relative body weight and human adiposity. Behavior genetics, 1997. 27(4):325-51.

9. Rankinen T, Zuberi A, Chagnon YC, Weisnagel SJ, Argyropoulos G, Walts B, Perusse L, Bouchard C. The human obesity gene map: the 2005 update. Obesity, 2006. 14(4):529-644.

10. Andreasen $\mathrm{CH}$, Stender-Petersen KL, Mogensen MS, Torekov SS, Wegner L, Andersen G et al. Low physical activity accentuates the effect of the FTO rs9939609 polymorphism on body fat accumulation. Diabetes, 2008. 57(1):95-101.

11. Cornes BK, Lind PA, Medland SE, Montgomery GW, Nyholt DR, Martin NG. Replication of the association of common rs9939609 variant of FTO with increased BMI in an Australian adult twin population but no evidence for gene by environment $(G \times E)$ interaction. International journal of obesity, 2009. 33(1):75-9.

12. Franks PW, Jablonski KA, Delahanty LM, McAteer JB, Kahn SE, Knowler WC, Florez JC, Diabetes Prevention Program Research G. Assessing gene-treatment interactions at the FTO and INSIG2 loci on obesity-related traits in the Diabetes Prevention Program. Diabetologia, 2008. 51(12):2214-23.

13. Qi L, Cho YA. Gene-environment interaction and obesity. Nutrition reviews, 2008. 66(12):684-94.

14. Vogels N, Posthumus DL, Mariman EC, Bouwman F, Kester AD, Rump P, Hornstra G, WesterterpPlantenga MS. Determinants of overweight in a cohort of Dutch children. The American journal of clinical nutrition, 2006. 84(4):717-24.

15. von Ruesten A, Steffen A, Floegel A, van der AD, Masala G, Tjonneland A et al. Trend in obesity prevalence in European adult cohort populations during follow-up since 1996 and their predictions to 2015. Plos One, 2011. 6(11):e27455.

16. Pi-Sunyer FX, Laferrere B, Aronne LJ, Bray GA. Therapeutic controversy: Obesity--a modern-day epidemic. The Journal of clinical endocrinology and metabolism, 1999. 84(1):3-12.

17. Marshall NS, Glozier N, Grunstein RR. Is sleep duration related to obesity? A critical review of the epidemiological evidence. Sleep medicine reviews, 2008. 12(4):289-98.

18. Horton ES. Effects of lifestyle changes to reduce risks of diabetes and associated cardiovascular risks: results from large scale efficacy trials. Obesity (Silver Spring), 2009. 17 Suppl 3:S43-8.

19. Van Gaal LF, Wauters MA, De Leeuw IH. The beneficial effects of modest weight loss on cardiovascular risk factors. Int J Obes Relat Metab Disord, 1997. 21 Suppl 1:S5-9.

20. Barte JC, ter Bogt NC, Bogers RP, Teixeira PJ, Blissmer B, Mori TA, Bemelmans WJ. Maintenance of weight loss after lifestyle interventions for overweight and obesity, a systematic review. Obes Rev, 2010. 11(12):899-906.

21. Wu T, Gao X, Chen M, van Dam RM. Long-term effectiveness of diet-plus-exercise interventions vs. diet-only interventions for weight loss: a meta-analysis. Obes Rev, 2009. 10(3):313-23.

22. Wing RR, Hill JO. Successful weight loss maintenance. Annu Rev Nutr, 2001. 21:323-41.

23. Purcell K, Sumithran P, Prendergast LA, Bouniu CJ, Delbridge E, Proietto J. The effect of rate of weight loss on long-term weight management: a randomised controlled trial. The lancet Diabetes \& endocrinology, 2014. 2(12):954-62.

24. Sumithran P, Prendergast LA, Delbridge E, Purcell K, Shulkes A, Kriketos A, Proietto J. Long-term persistence of hormonal adaptations to weight loss. The New England journal of medicine, 2011. 365(17):1597-604. 
25. Rosenbaum M, Nicolson M, Hirsch J, Murphy E, Chu F, Leibel RL. Effects of weight change on plasma leptin concentrations and energy expenditure. The Journal of clinical endocrinology and metabolism, 1997. 82(11):3647-54.

26. Ochner CN, Barrios DM, Lee CD, Pi-Sunyer FX. Biological mechanisms that promote weight regain following weight loss in obese humans. Physiology \& behavior, 2013. 120:106-13.

27. Rosenbaum M, Sy M, Pavlovich K, Leibel RL, Hirsch J. Leptin reverses weight loss-induced changes in regional neural activity responses to visual food stimuli. J Clin Invest, 2008. 118(7):2583-91.

28. Rosenbaum M, Murphy EM, Heymsfield SB, Matthews DE, Leibel RL. Low dose leptin administration reverses effects of sustained weight-reduction on energy expenditure and circulating concentrations of thyroid hormones. The Journal of clinical endocrinology and metabolism, 2002. 87(5):2391-4.

29. Karmali S, Brar B, Shi X, Sharma AM, de Gara C, Birch DW. Weight recidivism post-bariatric surgery: a systematic review. Obesity surgery, 2013. 23(11):1922-33.

30. Elfhag K, Rossner S. Who succeeds in maintaining weight loss? A conceptual review of factors associated with weight loss maintenance and weight regain. Obesity reviews : an official journal of the International Association for the Study of Obesity, 2005. 6(1):67-85.

31. Cinti S. The adipose organ at a glance. Disease models \& mechanisms, 2012. 5(5):588-94.

32. Rutkowski JM, Stern JH, Scherer PE. The cell biology of fat expansion. The Journal of cell biology, 2015. 208(5):501-12.

33. Arner P, Spalding KL. Fat cell turnover in humans. Biochemical and biophysical research communications, 2010. 396(1):101-4.

34. Spalding KL, Arner E, Westermark PO, Bernard S, Buchholz BA, Bergmann O et al. Dynamics of fat cell turnover in humans. Nature, 2008. 453(7196):783-7.

35. Farnier C, Krief S, Blache M, Diot-Dupuy F, Mory G, Ferre P, Bazin R. Adipocyte functions are modulated by cell size change: potential involvement of an integrin/ERK signalling pathway. International journal of obesity and related metabolic disorders : journal of the International Association for the Study of Obesity, 2003. 27(10):1178-86.

36. Bluher M, Mantzoros CS. From leptin to other adipokines in health and disease: facts and expectations at the beginning of the 21st century. Metabolism: clinical and experimental, 2015. 64(1):131-45.

37. Rosenbaum M, Goldsmith R, Bloomfield D, Magnano A, Weimer L, Heymsfield S, Gallagher D, Mayer L, Murphy $\mathrm{E}$, Leibel RL. Low-dose leptin reverses skeletal muscle, autonomic, and neuroendocrine adaptations to maintenance of reduced weight. The Journal of clinical investigation, 2005. 115(12):3579-86.

38. Crujeiras AB, Goyenechea E, Abete I, Lage M, Carreira MC, Martinez JA, Casanueva FF. Weight regain after a diet-induced loss is predicted by higher baseline leptin and lower ghrelin plasma levels. J Clin Endocrinol Metab, 2010. 95(11):5037-44.

39. Capel F, Klimcakova E, Viguerie N, Roussel B, Vitkova M, Kovacikova M et al. Macrophages and adipocytes in human obesity: adipose tissue gene expression and insulin sensitivity during calorie restriction and weight stabilization. Diabetes, 2009. 58(7):1558-67.

40. Gummesson A, Jernas M, Svensson PA, Larsson I, Glad CA, Schele E et al. Relations of adipose tissue CIDEA gene expression to basal metabolic rate, energy restriction, and obesity: population-based and dietary intervention studies. The Journal of clinical endocrinology and metabolism, 2007. 92(12):475965.

41. Viguerie N, Vidal H, Arner P, Holst C, Verdich C, Avizou S et al. Adipose tissue gene expression in obese subjects during low-fat and high-fat hypocaloric diets. Diabetologia, 2005. 48(1):123-31.

42. Huang P, Li S, Shao M, Qi Q, Zhao F, You J, Mao T, Li W, Yan Z, Liu Y. Calorie restriction and endurance exercise share potent anti-inflammatory function in adipose tissues in ameliorating diet-induced obesity and insulin resistance in mice. Nutrition \& metabolism, 2010. 7:59.

43. Kern PA, Saghizadeh M, Ong JM, Bosch RJ, Deem R, Simsolo RB. The expression of tumor necrosis factor in human adipose tissue. Regulation by obesity, weight loss, and relationship to lipoprotein lipase. The Journal of clinical investigation, 1995. 95(5):2111-9.

44. Palming J, Sjoholm K, Jernas M, Lystig TC, Gummesson A, Romeo S, Lonn L, Lonn M, Carlsson B, Carlsson LM. The expression of $\mathrm{NAD}(\mathrm{P}) \mathrm{H}$ :quinone oxidoreductase 1 is high in human adipose tissue, reduced by weight loss, and correlates with adiposity, insulin sensitivity, and markers of liver dysfunction. The Journal of clinical endocrinology and metabolism, 2007. 92(6):2346-52.

45. Bjorntorp P, Carlgren G, Isaksson B, Krotkiewski M, Larsson B, Sjostrom L. Effect of an energy-reduced dietary regimen in relation to adipose tissue cellularity in obese women. The American journal of clinical nutrition, 1975. 28(5):445-52. 
46. Lofgren P, Hoffstedt J, Naslund E, Wiren M, Arner P. Prospective and controlled studies of the actions of insulin and catecholamine in fat cells of obese women following weight reduction. Diabetologia, 2005. 48(11):2334-42.

47. Svensson PA, Gabrielsson BG, Jernas M, Gummesson A, Sjoholm K. Regulation of human aldoketoreductase 1C3 (AKR1C3) gene expression in the adipose tissue. Cellular \& molecular biology letters, 2008. 13(4):599-613.

48. Frantz C, Stewart KM, Weaver VM. The extracellular matrix at a glance. Journal of cell science, 2010. 123(Pt 24):4195-200.

49. DiGirolamo M, Fine JB, Tagra K, Rossmanith R. Qualitative regional differences in adipose tissue growth and cellularity in male Wistar rats fed ad libitum. The American journal of physiology, 1998. 274(5 Pt 2):R1460-7.

50. Mariman EC, Wang P. Adipocyte extracellular matrix composition, dynamics and role in obesity. Cell Mol Life Sci, 2010. 67(8):1277-92.

51. Faust IM, Johnson PR, Stern JS, Hirsch J. Diet-induced adipocyte number increase in adult rats: a new model of obesity. The American journal of physiology, 1978. 235(3):E279-86.

52. Arner P, Bernard S, Salehpour M, Possnert G, Liebl J, Steier P et al. Dynamics of human adipose lipid turnover in health and metabolic disease. Nature, 2011. 478(7367):110-3.

53. Eastman Q. Very low calorie diet makes adipocytes "scream". Journal of proteome research, 2009. 8(12):5408.

54. Bouwman FG, Wang P, van Baak M, Saris WH, Mariman EC. Increased beta-oxidation with improved glucose uptake capacity in adipose tissue from obese after weight loss and maintenance. Obesity, 2014. 22(3):819-27. 


\section{Chapter 2}

\section{Variation in extracellular matrix genes is associated with weight regain after weight loss in a sex-specific manner}

Nadia J.T. Roumans, Roel G. Vink, Marij Gielen, Maurice P. Zeegers, Claus Holst, Ping Wang, Arne Astrup, Wim H. Saris, Armand Valsesia, Jörg Hager, Marleen A. van Baak and Edwin C.M. Mariman

Genes \& Nutrition, 2015, 10 (6): 56 


\begin{abstract}
The extracellular matrix (ECM) of adipocytes is important for body weight regulation. Here, we investigated whether genetic variation in ECM related genes is associated with weight regain among participants of the European DiOGenes study. Overweight and obese subjects ( $n=469,310$ females, 159 males) were on an 8-week low-calorie diet with a 6-month follow-up. Body weight was measured before and after the diet, and after follow-up. Weight maintenance scores (WMS, regained weight as percentage of lost weight) were calculated based on the weight data. Genotype data was retrieved for 2903 SNPs corresponding to 124 ECM-related genes. Regression analyses provided us with six significant SNPs associated with the WMS in males: 3 SNPs in the POSTN gene and a SNP in the $\angle A M B 1, C O L 23 A 1$ and FBLN5 genes. For females, 1 SNP was found in the FN1 gene. The risk for weight regain was increased by: the $C / C$ genotype for POSTN in a co-dominant model (OR 8.25, 95\% $\mathrm{Cl}$ 2.85-23.88) and the T/C-C/C genotype in a dominant model (OR 4.88, 95\% Cl 2.35-10.16); the A/A genotype for $\angle A M B 1$ both in a co-dominant model (OR 18.43,95\% $\mathrm{Cl} 2.35-144.63$ ) and in a recessive model (OR 16.36, 95\% Cl 2.14-124.9); the G/A genotype for COL23A1 in a co-dominant model (OR 3.94, 95\% Cl 1.28-12.10), or the A-allele in a dominant model (OR 2.86, 95\% Cl 1.10-7.49); the A/A genotype for FBLN5 in a co-dominant model (OR 13.00, 95\% Cl 1.61-104.81); the A/A genotype for FN1 in a recessive model (OR 2.81, 95\% Cl 1.40-5.63). Concluding, variants of ECM-genes are associated with weight regain after weight loss in a sex-specific manner.
\end{abstract}




\section{Introduction}

Overweight and obesity have become a worldwide public health problem, associated with increased risk for many health complications such as diabetes and cardiovascular disease (1). Such risk can be reduced by losing weight (2). However, remaining at a lower body weight after weight loss provides a challenge for many people (3). The white adipose tissue plays an important role in the body weight change $(4,5)$. Weight loss reduces the amount of visceral adiposity and fat mass $(6)$, indicating that adipose tissue is one of the important determinants in this process. It has been proposed that the adipose tissue is also involved in the risk for weight regain after weight loss. White adipocytes are characterized by the presence of a single fat droplet, which almost fills the entire cell, and are surrounded by a thick extracellular matrix (ECM) (7). The ECM, is known for providing structural support, but also for fulfilling vital roles in cell differentiation, such as the determination, proliferation, polarity, survival, and migration of cells $(8,9)$. Prior studies have noted the importance of the ECM in relation to weight regulation. It was shown that ECM-regulated processes are disturbed in obese mice and humans leading to the accumulation of immune cells in the adipose tissue, impaired metabolic function, and reduced capacity for fat mass expansion $(10,11)$. After longterm weight reduction, a down regulation of ECM-regulating genes and changes in expression levels of ECM components can be observed in adipose tissue (11-14). In addition, Tam et al. reported that $10 \%$ body weight gain causes an upregulation of ECM-remodelling genes in the adipose tissue of male subjects (15). When people decrease their energy intake and enter a negative energy balance, mature adipocytes decrease their fat content and become smaller (16). The ECM is supposed to adjust to changes in cell volume. It has been proposed that this may lead to an improper fit between the cell and the surrounding ECM, thereby inducing tension and cellular stress (17). This cellular stress in adipocytes may be reduced by restoring fat and increasing cell volume, which would mean regain of weight for the host $(17,18)$. If the ECM is able to adjust properly to the volume changes, less cellular stress is generated to result in lower risk for weight regain. In line with this view, the subcutaneous adipose tissue ECM gene expression after a low caloric diet has been reported with differences in weight regainers compared to weight maintainers (19). We hypothesized that variation in genes coding for components of the adipocyte ECM are candidates for determining the risk of weight regain or the successfulness of weight maintenance after weight loss. In the present study, we examined whether genetic variation in ECM-related genes is associated with weight regain among the participants of the European DiOGenes study. We analyzed the present data separately for males and females because gender specificity has been shown for ECM remodeling in rodents $(20,21)$ and humans $(22,23)$, although it has not been specifically examined in adipose tissue.

\section{Materials and Methods}

\section{Participants and study design}

Participants took part in a pan-European, multicentre, randomised controlled dietary intervention programme called DiOGenes (http://www.diogenes-eu.org, ClinicalTrials.gov registration no.: NCT00390637). The whole study design has been described in detail previously (24-26). This study was conducted in eight European countries: the Netherlands, Denmark, the United Kingdom, Greece, Bulgaria, Germany, Spain and the Czech Republic. For 8 weeks, healthy overweight or obese participants followed a low-calorie diet (LCD) that provided about 3.3-4.2 MJ/day which is between 800 and $1000 \mathrm{kcal} /$ day. After the diet, participants were randomly assigned to 1 of 4 ad libitum consumed low-fat weight-maintenance diets. These diets differed in glycemic index and protein 
content (25). Body weight and other physical and biochemical parameters were measured after overnight fasting on a calibrated scale before weight loss on clinical investigation day 1 (CID1, $t=0)$, after LCD on CID2 ( $t=8 w)$ and after weight maintenance on CID3 $(t=8 w+6 m)$. For the current analysis, only participants who provided weight measurements at all 3 investigation days and who were successfully genotyped were used. In total, 469 participants met these criteria. Weight maintenance scores were calculated for all 469 participants as previously described by Wang et al. (26):

$W M S=($ weight at CID3 - weight at CID2 $) \div($ weight at CID $1-$ weight at CID2 $)$

A score equal or lower than zero indicated that the participant maintained or continued to lose weight (WM) during the follow-up period, while a score higher than zero indicated that the participant regained weight (WR) during the 6 month follow-up.

\section{DNA extraction and genotyping}

Buffy coats of EDTA-blood were used to extract DNA for genotyping. Genotyping was done using the Illumina Bead Station System (Illuminalnc) by IntegraGen using the Illumina 660quad chip, which analyses 660.000 SNPs and CNVs per sample. Genotype QC was carried out for all SNPs and SNPs were excluded from the analysis if they had a call rate $<98 \%, M A F<0.01$ or were not in HWE. Centre d'Etude du Polymorphisme Humain control samples were added on each plate: one was different on each plate and one was identical among the 15 genotyped plates. The reproducibility was $100 \%$ and the concordance rate was $99.6 \%$. For the purpose of the present study, genotypes for all individuals were extracted from the genotyping matrix for the candidate SNPs only.

Based on the proteins detected in the adipocyte ECM (7), a list of 124 candidate genes related to the ECM was created (Supplement Table S1). Genotype data from the DiOGenes cohort was retrieved for 2903 SNPs (Supplement Table S2) in and near the 124 genes.

\section{Data analysis}

Dependant T-test was applied to check for differences between time points within a group. A chisquare test was used to check whether the genotype frequencies of the SNPs were in HardyWeinberg equilibrium.

Univariate linear regression analyses were carried out with each SNP allele as a predictor and weight maintenance scores as outcome. The analyses were done for males and females separately because of gender specificity in ECM remodelling found in other studies. Regression analyses were done with the use of Stata 12.0 (StataCorp LP). P-values were corrected for false discovery rate (FDR) in multiple testing with the Benjamini-Hochberg method with the 'stats' package in $\mathrm{R}$ (version 3.1.1; http://www.r-project.org/) (27). Corrected P-values $<0.05$ were considered to be statistically significant. Next, SNPs with a minor allele frequency $<5 \%$ were excluded to distinguish common polymorphism from rare variants. Linkage disequilibrium $r^{2}$-values $>0.2$ was used to determine if periostin SNPs were in linkage disequilibrium. Linkage disequilibrium structure was evaluated by using SNPStats (28).

Backward elimination in multivariate linear regression was used to check if combinations of SNPs might enhance the outcome. For this, the four significant SNPs observed in the male population were used. 
Genotype analyses: logistic regression analysis was used to find the best genetic model of inheritance that describes the effect of the genotypes of the significant SNPs. A model is considered best fitting if it has the lowest Akaike information criterion (AIC) score and if this value is at least 2 lower than the other models. If multiple models have similar low AIC values than these models are fitting equally well. Logistic regression, odds ratios (OR), 95\% confidence intervals $(95 \% \mathrm{Cl})$ were calculated to determine the risk of a specific genotype on weight regain. Genotype analyses were all carried out using Stata 12.0 (StataCorp LP).

\section{Results}

\section{Participant characteristics}

Participant characteristics of weight regainers and maintainers, separated for males and females, can be seen in Table 1. Comparisons between baseline and after 8 weeks show that weight, BMI, WC and fat mass (FM) significantly decreased after the 8 week LCD for all groups. After the 6 -month weight maintenance period, all parameters were significantly decreased for weight maintainers (WM) while a significant increase is observed for weight regainers (WR) when comparing to measurements after the LCD diet. Weight maintenance scores (WMS) were significantly different between WM and WR for males $(P<0.001)$ and females $(P<0.001)$.

\section{Single nucleotide polymorphisms univariate linear regression analyses}

The SNPs with a corrected P-value $<0.05$ are depicted in Table 2, and the results of all SNPs are shown in Supplement Table S1. Further selection on minor allele frequency (MAF) resulted for the male group in only 6 SNPs with an allele frequency higher than $5 \%$ (indicated in bold in Table 2): rs7323378, rs9315503, rs9547947, rs2158836, rs12589592, and rs2672826. Three of the SNPs are in and around the periostin gene (POSTN, rs7323378, rs9315503, rs9547947), the other SNPs are in the laminin- $\beta 1$ (LAMB1, rs2158836), fibulin-5 (FBLN5, rs12589592) and collagen, Type XXIII, alpha1 (COL23A1, rs2672826) genes. The three POSTN SNPs were all in linkage disequilibrium: rs 7323378 rs9547947 ( $\left.r^{2}=0.760\right)$, rs7323378 $-r s 9315503\left(r^{2}=0.487\right)$ and rs9547947 $-r s 9315503\left(r^{2}=0.370\right)$. It indicates that these variants are closely linked and therefore only the SNP with the lowest P-value was used for further analysis, which is POSTN rs7323378. For female subjects, 1 SNP remained after selecting SNPs with a minor allele frequency higher than 5\%: rs17516906. This SNP is located in the fibronectin 1 (FN1) gene.

\section{SNP Multivariate linear regression analyses}

Backward elimination in multivariate linear regression with the significant SNPs observed in the male population resulted in significance for three SNPs: rs2672826 $(\beta=-17.52, P=0.020)$, rs2158836 $(\beta=-$ 11.50, $\mathrm{P}=0.039)$ and rs7323378 $(\beta=-13.67, \mathrm{P}=0.009)$. This indicates that COL23A1, POSTN and LAMB1 together have an additive effect on weight regulation. 
$\sim$ Table 1: Changes in subject characteristics at the end of 8 week low-calorie diet compared to baseline, and at the end of 6-month ad libitum diet compared to the end of 8-week LCD.

\begin{tabular}{|c|c|c|c|c|c|c|c|c|c|c|c|c|}
\hline & \multicolumn{4}{|l|}{ Baseline } & \multicolumn{4}{|c|}{ After 8-week LCD } & \multicolumn{4}{|c|}{ After 6-month ad libitum diet } \\
\hline & \multicolumn{2}{|l|}{ Male } & \multicolumn{2}{|l|}{ Female } & \multicolumn{2}{|l|}{ Male } & \multicolumn{2}{|l|}{ Female } & \multicolumn{2}{|l|}{ Male } & \multicolumn{2}{|l|}{ Female } \\
\hline & WM & WR & WM & WR & WM & WR & WM & WR & WM & WR & WM & WR \\
\hline $\mathrm{N}$ & 59 & 100 & 135 & 175 & 59 & 100 & 135 & 175 & 59 & 100 & 135 & 175 \\
\hline Age $(y)$ & $43 \pm 6$ & $43 \pm 6$ & $41 \pm 7$ & $42 \pm 6$ & & & & & & & & \\
\hline Weight (kg) & $111.3 \pm 18.5$ & $107.9 \pm 17.1$ & $97.3 \pm 18.3$ & $92.1 \pm 13.1$ & $97.7 \pm 17.3^{*}$ & $95.3 \pm 15.0^{*}$ & $86.3 \pm 16.6^{*}$ & $82.6 \pm 12^{*}$ & $93.5 \pm 17.5 \#$ & $99.8 \pm 15.7 \#$ & $81.6 \pm 15.1 \#$ & $86.2 \pm 12.6 \#$ \\
\hline $\mathrm{BMI}\left(\mathrm{kg} / \mathrm{m}^{2}\right)$ & $35.4 \pm 4.9$ & $33.4 \pm 4.6$ & $35.1 \pm 5.4$ & $33.5 \pm 4.4$ & $31.1 \pm 4.6^{*}$ & $29.6 \pm 4.1^{*}$ & $31.1 \pm 4.8^{*}$ & $30.1 \pm 4.0^{*}$ & $29.8 \pm 4.7 \#$ & $30.9 \pm 4.3 \#$ & $29.5 \pm 4.5 \#$ & $31.4 \pm 4.2 \#$ \\
\hline WC (cm) & $117.4 \pm 12.9$ & $111.9 \pm 11.8$ & $104.9 \pm 13.6$ & $102.3 \pm 10.4$ & $105.4 \pm 12.7^{*}$ & $100.5 \pm 11.5^{*}$ & $95.2 \pm 12.3^{*}$ & $93.6 \pm 10.1 *$ & $101 \pm 12.4 \#$ & $104.5 \pm 12.0 \#$ & $91.8 \pm 12.2 \#$ & $96.4 \pm 10.1 \#$ \\
\hline Fat mass (kg) & $39.6 \pm 13.4$ & $34.1 \pm 10.7$ & $44 \pm 12$ & $40.8 \pm 9.3$ & $30.4 \pm 10.6^{*}$ & $26.7 \pm 10.1^{*}$ & $35.3 \pm 10.9^{*}$ & $33.8 \pm 9.3^{*}$ & $\begin{array}{l}25.3 \pm 10.6 \# \\
-0.33+0.34\end{array}$ & $28.4 \pm 10.3 \#$ & $\begin{array}{l}30.5 \pm 9.4 \# \\
-0.42+0.39\end{array}$ & $35.6 \pm 9.2 \#$ \\
\hline
\end{tabular}

Values are means \pm SD. Subjects are divided into 4 groups: male weight maintainers (WM, $n=59)$, weight regainers (WR, $n=100)$ and female WM ( $n=135)$ and WR $(n=175)$. Weight maintenance score (WMS) is calculated at the end of the 6-month ad libitum diet: (weight after 6-month ad libitum diet - weight after LCD) / (weight at baseline - weight after LCD).

WC, waist circumference; LCD, low-calorie diet; WMS, weight maintenance score.

$* \mathrm{P}<0.001$ change from baseline vs. after 8 week LCD with dependant T-test per group.

$\#$ \# $<0.001$ change from after 8 week LCD vs. after 6 month ad libitum diet with dependent T-test per group. 
Table 2: Regression analyses carried out with each SNP allele as a predictor and weight maintenance scores as outcome separately for males and females.

\begin{tabular}{|c|c|c|c|c|c|c|}
\hline SNP & Gene & No. of subjects & P-value & $\mathbf{P}_{\text {corr }}$ & Minor allel & MAF \% \\
\hline \multicolumn{7}{|l|}{ Males } \\
\hline rs8031741 & ACAN & 159 & $9.57 E-26$ & $<0.001$ & G & 0.3 \\
\hline rs2271649 & ADAM12 & 158 & $5.12 \mathrm{E}-07$ & $<0.001$ & A & 0.9 \\
\hline rs16859850 & CCDC80 & 158 & $4.08 \mathrm{E}-17$ & $<0.001$ & G & 0.6 \\
\hline rs2300792 & COL12A1 & 157 & $1.43 \mathrm{E}-22$ & $<0.001$ & C & 0.3 \\
\hline rs16918099 & COL15A1 & 159 & $3.29 \mathrm{E}-05$ & 0.005 & A & 0.2 \\
\hline rs16918124 & COL15A1 & 159 & $6.19 \mathrm{E}-05$ & 0.008 & C & 0.5 \\
\hline rs7863250 & COL15A1 & 159 & $6.19 \mathrm{E}-05$ & 0.008 & C & 0.5 \\
\hline rs2672826 & COL23A1 & 157 & 4.74E-04 & 0.048 & A & 12.1 \\
\hline rs12477499 & COL3A1 & 159 & $2.49 \mathrm{E}-14$ & $<0.001$ & G & 0.3 \\
\hline rs12589592 & FBLN5 & 159 & 2.13E-04 & 0.023 & A & 33.1 \\
\hline rs12050562 & FBN1 & 159 & $8.75 \mathrm{E}-05$ & 0.010 & $\mathrm{~T}$ & 0.8 \\
\hline rs7606877 & GPC1 & 159 & $1.41 \mathrm{E}-14$ & $<0.001$ & A & 0.5 \\
\hline rs9492168 & LAMA2 & 158 & $7.50 \mathrm{E}-17$ & $<0.001$ & $\mathrm{~T}$ & 0.3 \\
\hline rs2158836 & LAMB1 & 159 & 1.75E-04 & 0.020 & A & 37.1 \\
\hline rs10911215 & LAMC1 & 159 & $1.11 \mathrm{E}-09$ & $<0.001$ & $\mathrm{~T}$ & 1.3 \\
\hline rs2513812 & MATN2 & 159 & 4.36E-09 & $<0.001$ & G & 1.3 \\
\hline rs1151578 & NID2 & 158 & $2.53 \mathrm{E}-08$ & $<0.001$ & $\mathrm{~T}$ & 0.2 \\
\hline rs6480654 & P4HA1 & 157 & $2.67 \mathrm{E}-12$ & $<0.001$ & A & 0.2 \\
\hline rs1382192 & PDIA4 & 159 & $4.90 \mathrm{E}-13$ & $<0.001$ & $A$ & 0.5 \\
\hline rs4727007 & PDIA4 & 159 & $4.90 E-13$ & $<0.001$ & G & 0.5 \\
\hline rs10197695 & PDIA6 & 159 & $1.26 \mathrm{E}-14$ & $<0.001$ & G & 0.3 \\
\hline rs7323378 & POSTN & 158 & $1.10 \mathrm{E}-05$ & 0.002 & C & 48.0 \\
\hline rs9547947 & POSTN & 149 & $2.62 \mathrm{E}-05$ & 0.004 & A & 38.3 \\
\hline rs9315503 & POSTN & 159 & $2.20 \mathrm{E}-04$ & 0.023 & $\mathbf{G}$ & 33.9 \\
\hline \multicolumn{7}{|l|}{ Females } \\
\hline rs7679471 & TLL1 & 159 & $3.36 \mathrm{E}-07$ & $<0.001$ & C & 0.2 \\
\hline rs8031741 & ACAN & 309 & $5.14 \mathrm{E}-16$ & 0.000 & G & 0.3 \\
\hline rs4871046 & COL14A1 & 307 & 5.49E-09 & 0.000 & C & 0.5 \\
\hline rs16918099 & COL15A1 & 310 & $5.75 E-44$ & 0.000 & A & 0.2 \\
\hline rs12477499 & COL3A1 & 310 & $2.19 E-43$ & 0.000 & G & 0.3 \\
\hline rs17516906 & FN1 & 303 & $1.28 \mathrm{E}-04$ & 0.040 & G & 7.4 \\
\hline rs1151578 & NID2 & 310 & 5.19E-06 & 0.002 & $\mathrm{~T}$ & 0.2 \\
\hline rs11925421 & PLOD2 & 310 & $7.20 \mathrm{E}-10$ & 0.000 & G & 0.6 \\
\hline rs7078493 & TLL2 & 310 & 5.19E-06 & 0.000 & $\mathrm{~T}$ & 0.2 \\
\hline rs310517 & VCAN & 310 & $1.79 \mathrm{E}-25$ & 0.000 & $\mathrm{~T}$ & 0.5 \\
\hline
\end{tabular}

$\mathrm{P}$-values are derived from univariate linear regression analyses and $\mathrm{P}_{\text {corr }}$ are $\mathrm{P}$-values corrected for false discovery rate.

$\mathrm{P}_{\text {corr }} \leq 0.05$ are considered significant, SNPs with a MAF $<5 \%$ are excluded. SNPs in bold have $\mathrm{P}_{\text {corr }} \leq 0.05$ and MAF $>5 \%$. MAF, minor allel frequency. 


\section{Genotype analyses}

Genotype analyses were done to get an idea of the effect of specific genotypes on weight change. Figure 1 shows the comparison between number of genotypes of weight maintainers (blue bars) and weight regainers (white bars) for the significant SNPs. Table 3 shows the best model of fit and the associations between genotypes and the risk for weight regain. For COL23A1 rs2672826, the best fitting models are the co-dominant and dominant model. In the co-dominant model, it is seen that a G/A genotype increases the risk for weight regain by 3.9 times compared to a $G / G$ genotype (OR 3.94, 95\% $\mathrm{Cl}$ 1.28-12.10). The dominant model indicates that the $G / A-A / A$ genotype increases the risk for weight regain by 2.9-fold compared to the $\mathrm{G} / \mathrm{G}$ genotype (OR $2.86,95 \% \mathrm{Cl} 1.10-7.49$ ). The best fitting model for FBLN5 rs 12589592 is the co-dominant model with a 13-fold increased risk for weight regain with an A/A genotype (OR $13.00,95 \% \mathrm{Cl} 1.61-104.81$ ) and a 2.3-fold increase with a G/A genotype (OR 2.26, 95\% Cl 1.15-4.46) compared to a $G / G$ genotype. The best fitting models for $\angle A M B 1$ rs2158836 are the co-dominant and the recessive model. In the co-dominant model an A/A genotype gives an 18.4 times higher chance for weight regain than a $\mathrm{G} / \mathrm{G}$ genotype (OR $18.43,95 \% \mathrm{Cl}$ 2.35-144.63). In the recessive model this risk is increased 16.4 times (OR 16.36, 95\% Cl 2.14-124.9). The best fitting models for POSTN rs7323378 are the co-dominant as well as the dominant model. The chance for weight regain is increased 8.3 times with the $\mathrm{C} / \mathrm{C}$ genotype in the co-dominant model (OR 8.25, 95\% $\mathrm{Cl}$ 2.85-23.88) when compared with a T/T genotype. In the dominant model, a T/C-C/C genotype increases the risk for weight regain by 4.9 times (OR $4.88,95 \% \mathrm{Cl} 2.35-10.16$ ). The codominant model and recessive model are best fitting for the FN1 rs17516906 SNP but a correct comparison in the co-dominant model cannot be made due to absence of $G / G$ genotypes for participants regaining weight during follow-up. The recessive model shows that an A/A genotype increase the risk for weight regain 2.8 times ( $O R 2.81,95 \% \mathrm{Cl} 1.40-5.63$ ) compared to a $\mathrm{G} / \mathrm{G}-\mathrm{A} / \mathrm{G}$ genotype. 
A

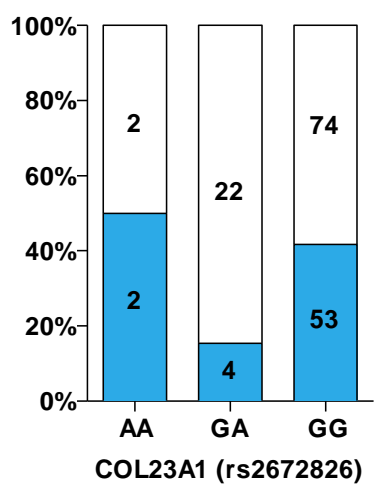

\section{C}

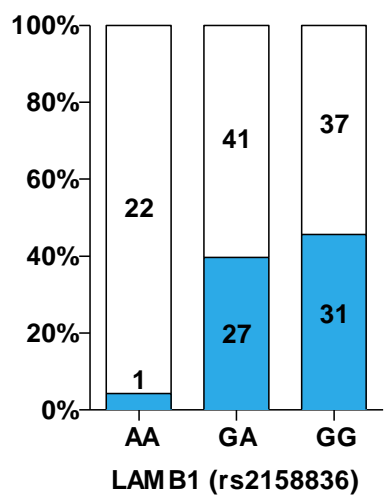

B

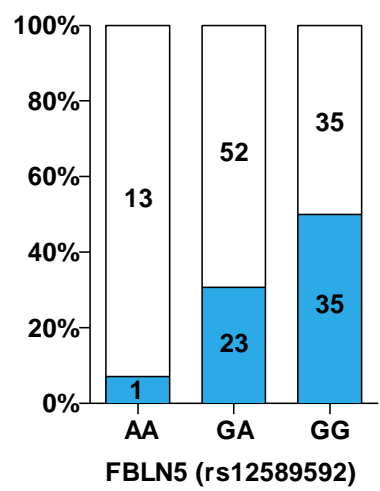

D

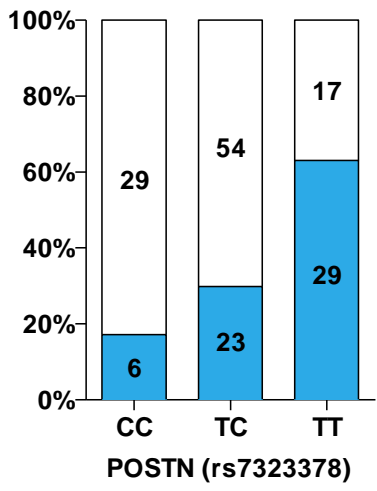

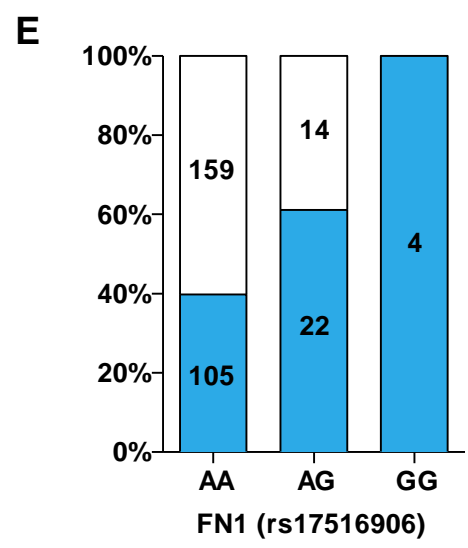

Figure 1: Percentage weight regain or maintenance phenotype of each genotype for the significant SNPs. Each bar represents the total amount of subjects having a specific genotype for a significant SNP: A COL23A1 (rs2672826), B FBLN5 (rs12589592), C LAMB1 (rs2158836), D POSTN (rs7323378) and E FN1 (rs17516906). A-D represent men and E represents women. The blue bars indicate the percentage of weight maintainers (WM) with the genotype and the white bars are for weight regainers (WR). The number within each bar is the count of participants having the specific genotype. 
Table 3: Logistic regression analyses used to find the best genetic model of inheritance that describes the effect of the genotypes.

\begin{tabular}{|c|c|c|c|c|}
\hline SNP & Model & Genotype & OR $(95 \% \mathrm{Cl})$ & AIC \\
\hline \multirow{7}{*}{$\begin{array}{l}\text { rs2672826 } \\
A>G \\
\text { COL23A1 }\end{array}$} & \multirow[t]{3}{*}{ Co-dominant } & $\mathrm{G} / \mathrm{G}$ & 1.00 & \multirow{3}{*}{206.4} \\
\hline & & $\mathrm{G} / \mathrm{A}$ & $3.94(1.28-12.10)^{*}$ & \\
\hline & & $\mathrm{A} / \mathrm{A}$ & $0.72(0.10-5.25)$ & \\
\hline & \multirow[t]{2}{*}{ Dominant } & $\mathrm{G} / \mathrm{G}$ & 1.00 & \multirow{2}{*}{206.6} \\
\hline & & $\mathrm{G} / \mathrm{A}-\mathrm{A} / \mathrm{A}$ & $2.86(1.10-7.49)^{*}$ & \\
\hline & \multirow[t]{2}{*}{ Recessive } & G/G-G/A & 1.00 & \multirow{2}{*}{211.6} \\
\hline & & $\mathrm{A} / \mathrm{A}$ & $0.59(0.08-4.33)$ & \\
\hline \multirow{7}{*}{$\begin{array}{l}\text { rs12589592 } \\
\text { G > A } \\
\text { FBLN5 }\end{array}$} & \multirow[t]{3}{*}{ Co-dominant } & $\mathrm{G} / \mathrm{G}$ & 1.00 & \multirow{3}{*}{202.7} \\
\hline & & $\mathrm{G} / \mathrm{A}$ & $2.26(1.15-4.46)^{*}$ & \\
\hline & & $\mathrm{A} / \mathrm{A}$ & $13.00(1.61-104.81)^{*}$ & \\
\hline & \multirow[t]{2}{*}{ Dominant } & $\mathrm{G} / \mathrm{G}$ & 1.00 & \multirow{2}{*}{204.8} \\
\hline & & $\mathrm{G} / \mathrm{A}-\mathrm{A} / \mathrm{A}$ & $2.71(1.40-5.25)^{*}$ & \\
\hline & \multirow[t]{2}{*}{ Recessive } & $\mathrm{G} / \mathrm{G}-\mathrm{G} / \mathrm{A}$ & 1.00 & \multirow{2}{*}{206.4} \\
\hline & & $\mathrm{A} / \mathrm{A}$ & $8.67(1.10-68.06)^{*}$ & \\
\hline \multirow{7}{*}{$\begin{array}{l}\text { rs } 2158836 \\
A>G \\
\text { LAMB1 }\end{array}$} & \multirow{3}{*}{ Co-dominant } & $\mathrm{G} / \mathrm{G}$ & 1.00 & \multirow{3}{*}{199.3} \\
\hline & & $\mathrm{G} / \mathrm{A}$ & $1.27(0.64-2.51)$ & \\
\hline & & $\mathrm{A} / \mathrm{A}$ & $18.43(2.35-144.63)^{*}$ & \\
\hline & \multirow[t]{2}{*}{ Dominant } & $\mathrm{G} / \mathrm{G}$ & 1.00 & \multirow{2}{*}{210.1} \\
\hline & & $\mathrm{G} / \mathrm{A}-\mathrm{A} / \mathrm{A}$ & $1.89(0.98-3.62)^{*}$ & \\
\hline & \multirow[t]{2}{*}{ Recessive } & $\mathrm{G} / \mathrm{G}-\mathrm{G} / \mathrm{A}$ & 1.00 & \multirow{2}{*}{197.8} \\
\hline & & $\mathrm{A} / \mathrm{A}$ & $16.36(2.14-124.9)^{*}$ & \\
\hline \multirow{7}{*}{$\begin{array}{l}\text { rs7323378 } \\
T>C \\
\text { POSTN }\end{array}$} & \multirow[t]{3}{*}{ Co-dominant } & $\mathrm{T} / \mathrm{T}$ & 1.00 & \multirow{3}{*}{192.6} \\
\hline & & $\mathrm{T} / \mathrm{C}$ & $4.01(1.85-8.67)$ & \\
\hline & & $\mathrm{C} / \mathrm{C}$ & $8.25(2.85-23.88)^{*}$ & \\
\hline & \multirow[t]{2}{*}{ Dominant } & $\mathrm{T} / \mathrm{T}$ & 1.00 & \multirow{2}{*}{192.7} \\
\hline & & $\mathrm{T} / \mathrm{C}-\mathrm{C} / \mathrm{C}$ & $4.88(2.35-10.16)^{*}$ & \\
\hline & \multirow[t]{2}{*}{ Recessive } & $\mathrm{T} / \mathrm{T}-\mathrm{T} / \mathrm{C}$ & 1.00 & \multirow{2}{*}{203.6} \\
\hline & & $\mathrm{C} / \mathrm{C}$ & $3.54(1.37-9.14)^{*}$ & \\
\hline rs17516906 & \multirow[t]{3}{*}{ Co-dominant } & $\mathrm{G} / \mathrm{G}$ & 1.00 & \\
\hline$A>G$ & & $\mathrm{~A} / \mathrm{G}$ & 0.00 (NA) & 409.0 \\
\hline FN1 & & $A / A$ & 0.00 (NA) & \\
\hline & Dominant & $\mathrm{G} / \mathrm{G}$ & 1.00 & 4128 \\
\hline & & $\mathrm{A} / \mathrm{G}-\mathrm{A} / \mathrm{A}$ & 0.00 (NA) & 412.0 \\
\hline & Recessive & G/G-A/G & 1.00 & 7 \\
\hline & & $A / A$ & $2.81(1.40-5.63)^{*}$ & 410.1 \\
\hline
\end{tabular}

Odds ratio, 95\% confidence interval, Akaike information criterion (AIC) and P-values were retrieved from logistic regression analyses with the co-dominant, dominant, and recessive model. The best fitting model has the lowest AIC and this value is at least 2 lower than the other models. $\mathrm{P}$-value $\leq 0.05$ are considered significant ( $\left.{ }^{*}\right)$. OR, odds ratio; $\mathrm{Cl}$, confidence interval; NA, Not Available. 


\section{Discussion}

The major finding of this study is that variants of the POSTN, LAMB1, COL23A1 and FBLN5 gene for males and the FN1 gene for females can influence the risk for weight regain. To the best of our knowledge this is the first study that relates genetic variation of ECM genes with the risk for weight regain after weight loss. As such, our findings are in keeping with the proposed prominent role of the ECM in the adipocyte cellular stress model for weight regain (17).

For most of the identified genes or members of the same gene family, links with human weight regulation have been reported. POSTN is highly expressed in collagen-rich connective tissue such as adipose tissue. It has been related to obesity by Bolton et al. who observed higher POSTN expression in the adipose tissue of obese diabetic sand rates (Psammomys obesus) compared to healthy lean animals suggesting that POSTN may influence fat storage in adipocytes (29). High expression of POSTN was found in visceral as well as subcutaneous adipose tissue depots and a role in repair or expansion of the adipose tissue was suggested (29). Proper biomechanical function of connective tissue seems to depend on the interaction of POSTN and specific collagens (30). In POSTN-knockout mice, a decreased collagen cross-linking was observed and the mechanical stabilization of ECM architecture was disrupted (30). Here we show that in humans the $\mathrm{C} / \mathrm{C}$ genotype of the POSTN SNP rs7323378 increases the risk for weight regain after weight loss, which supports a role for the ECM in human weight regulation.

Laminins constitute a family of 12 genes coding for ECM components, which are subdivided in five $\alpha$-, four $\beta$-, and three $\gamma$-genes. SNPs in the LAMA5 gene have been associated with adiposity parameters in European and African Americans (31). In addition, a rare variant with a moderate-to-high predicted biological effect was detected in LAMC1 or LAMC3 in 5 of 30 extremely obese subjects (32). In the present study, we observed that the minor A/A genotype for the LAMB1 SNP increases the chance of weight regain after weight loss. Laminins actually form trimers from an $\alpha, \beta$ and $\gamma$ protein. Of all possible combinations, only fifteen different trimers have been observed in vivo. LAMB1 is a component of six of those fifteen, where it occurs in combination with LAMC1 or LAMC3.

Collagen XXIII belongs to the non-fibrillar transmembranous subfamily of collagens and as such it can be involved in cell-matrix contact, but concrete information about its function is lacking. Collagen XXIII has structural features in common with collagen XIII and may therefore have a similar function $(33,34)$. Type XIII collagen is expressed in almost all connective tissue producing cells $(35)$, and is important for the regulation of adhesion of mesenchymal cells to the surrounding ECM and neighbouring cells, thereby facilitating transmembrane signalling (36). In addition, data suggests the involvement of collagen XIII in numerous differentiation and maturation processes associated with inflammation and vasculogenesis (37). Positive correlations between inflammatory markers and weight regain after energy restriction have been reported (38). Together with our findings, it suggests that people with a G/A genotype for COL23A1 are predisposed to regaining weight due to differences in ECM biosynthesis and inflammatory profile.

Studies of the skin have revealed that collagen XXIII can bind as a ligand to integrin $\alpha_{2} \beta_{1}$, which directly interacts with the LAMB1-containing trimer laminin111 (39). In fact, FBLN5 also binds to $\beta$ integrin and in the mouse, it was observed that FBLN5 competes with FN for binding to integrin- $\beta 1$ (40). Altogether, four of the five genes identified here seem to interact with integrin- $\beta$, which 
suggests that integrins may play a key role in the influence of the ECM on weight regain. Moreover, a sex-specific preference for interaction with integrin- $\beta 1$ could explain our result that in men variation in the FBLN5 gene and in women variation in the FN1 gene is associated with weight regain after weight loss.

Among females, we observed that an A/A genotype in the FN1 gene is associated with weight regain. Our finding is in line with that of Mutch et al. (19) who demonstrated that FN1 was upregulated in subjects regaining weight after weight loss while the opposite was observed in subjects maintaining their lost weight. Fibronectin, an important component of the ECM, functions both as regulator of various cellular processes and as scaffolding protein maintaining and directing tissue organization and ECM composition (41). In morbid obese subjects elevated plasma levels of fibronectin are found and when these subjects lose weight, fibronectin levels normalise (42). During adipocyte differentiation, fibronectin levels decrease (43) and culturing preadipocytes on fibronectin-coated dishes prevents adipocyte differentiation (44). In this respect, fibronectin seems to influence the differentiation potential of preadipocytes. Although weight regain after weight loss is supposed to be mainly due to renewed fat accumulation in mature adipocytes $(14,17,18)$, to some extent differentiation of preadipocytes can also occur (45). Therefore, the predisposition to weight regain after weight loss mediated by the detected genetic variation in ECM genes may in part result from interference with volume changes of adipocytes and in part from influencing differentiation of preadipocytes.

Although DiOGenes is one of the largest weight loss-maintenance studies, a limitation of the current study is the relatively small number of participants. Because of this limited number, we did not take the variation of the maintenance diet into account. Genotype analysis of the total cohort of 469 subjects did not lead to significant results. Also, no functional analysis of the detected genetic variation was performed. Yet, this study produces leads for understanding the role of the ECM and its genetic variation in weight regain after weight loss, which should be confirmed and can be extended in larger cohorts.

In conclusion, we have investigated the role of genetic variation in ECM genes in regard to weight regain. Our results illustrate an involvement of variants of the POSTN, LAMB1, COL23A1, and FBLN5 gene for males and of the $F N 1$ gene for females in the risk for weight regain after weight loss. Influence on weight regulation may come from the level of cell stress generated between the ECM and the adipocyte during weight loss, but also from modifying the differentiation of preadipocytes. Further research is required to confirm our findings in a larger cohort and to bring more clarity in the mechanism of weight regain after weight loss. 


\section{References}

1. Smyth S, Heron A. Diabetes and obesity: the twin epidemics. Nat Med, 2006. 12(1):75-80.

2. Bessesen DH. Update on obesity. J Clin Endocrinol Metab, 2008. 93(6):2027-34.

3. Wing RR, Phelan S. Long-term weight loss maintenance. Am J Clin Nutr, 2005. 82(1 Suppl):222S-5S.

4. Balistreri CR, Caruso C, Candore G. The role of adipose tissue and adipokines in obesity-related inflammatory diseases. Mediators Inflamm, 2010. 2010:802078.

5. Bluher M. Adipose tissue dysfunction contributes to obesity related metabolic diseases. Best Pract Res Clin Endocrinol Metab, 2013. 27(2):163-77.

6. Stallone DD, Stunkard AJ, Wadden TA, Foster GD, Boorstein J, Arger P. Weight loss and body fat distribution: a feasibility study using computed tomography. Int J Obes, 1991. 15(11):775-80.

7. Mariman EC, Wang P. Adipocyte extracellular matrix composition, dynamics and role in obesity. Cell Mol Life Sci, 2010. 67(8):1277-92.

8. Hynes RO. The extracellular matrix: not just pretty fibrils. Science, 2009. 326(5957):1216-9.

9. Rozario T, DeSimone DW. The extracellular matrix in development and morphogenesis: a dynamic view. Dev Biol, 2010. 341(1):126-40.

10. Strissel KJ, Stancheva Z, Miyoshi H, Perfield JW, 2nd, DeFuria J, Jick Z, Greenberg AS, Obin MS. Adipocyte death, adipose tissue remodeling, and obesity complications. Diabetes, 2007. 56(12):29108.

11. Divoux A, Tordjman J, Lacasa D, Veyrie N, Hugol D, Aissat A et al. Fibrosis in human adipose tissue: composition, distribution, and link with lipid metabolism and fat mass loss. Diabetes, 2010. 59(11):2817-25.

12. Henegar C, Tordjman J, Achard V, Lacasa D, Cremer I, Guerre-Millo M et al. Adipose tissue transcriptomic signature highlights the pathological relevance of extracellular matrix in human obesity. Genome Biol, 2008. 9(1):R14.

13. Kos K, Wong S, Tan B, Gummesson A, Jernas M, Franck N et al. Regulation of the fibrosis and angiogenesis promoter SPARC/osteonectin in human adipose tissue by weight change, leptin, insulin, and glucose. Diabetes, 2009. 58(8):1780-8.

14. Kolehmainen M, Salopuro T, Schwab US, Kekalainen J, Kallio P, Laaksonen DE et al. Weight reduction modulates expression of genes involved in extracellular matrix and cell death: the GENOBIN study. Int J Obes (Lond), 2008. 32(2):292-303.

15. Tam CS, Covington JD, Bajpeyi S, Tchoukalova Y, Burk D, Johannsen DL, Zingaretti CM, Cinti S, Ravussin E. Weight gain reveals dramatic increases in skeletal muscle extracellular matrix remodeling. J Clin Endocrinol Metab, 2014. 99(5):1749-57.

16. Andersson DP, Eriksson Hogling D, Thorell A, Toft E, Qvisth V, Naslund E et al. Changes in Subcutaneous Fat Cell Volume and Insulin Sensitivity After Weight Loss. Diabetes Care, 2014.

17. Mariman EC. An adipobiological model for weight regain after weight loss. Adipobiology, 2011. 3(1):915.

18. Mariman EC. Human biology of weight maintenance after weight loss. J Nutrigenet Nutrigenomics, 2012. 5(1):13-25.

19. Mutch DM, Pers TH, Temanni MR, Pelloux V, Marquez-Quinones A, Holst C et al. A distinct adipose tissue gene expression response to caloric restriction predicts 6-mo weight maintenance in obese subjects. Am J Clin Nutr, 2011. 94(6):1399-409.

20. Lu H, Melendez GC, Levick SP, Janicki JS. Prevention of adverse cardiac remodeling to volume overload in female rats is the result of an estrogen-altered mast cell phenotype. Am J Physiol Heart Circ Physiol, 2012. 302(3):H811-7.

21. Salimena MC, Lagrota-Candido J, Quirico-Santos T. Gender dimorphism influences extracellular matrix expression and regeneration of muscular tissue in mdx dystrophic mice. Histochem Cell Biol, 2004. 122(5):435-44.

22. Komosinska-Vassev K, Olczyk P, Winsz-Szczotka K, Kuznik-Trocha K, Klimek K, Olczyk K. Age- and gender-dependent changes in connective tissue remodeling: physiological differences in circulating MMP-3, MMP-10, TIMP-1 and TIMP-2 level. Gerontology, 2011. 57(1):44-52.

23. Bowers SL, Banerjee I, Baudino TA. The extracellular matrix: at the center of it all. J Mol Cell Cardiol, 2010. 48(3):474-82.

24. Larsen TM, Dalskov S, van Baak M, Jebb S, Kafatos A, Pfeiffer A et al. The Diet, Obesity and Genes (Diogenes) Dietary Study in eight European countries - a comprehensive design for long-term intervention. Obes Rev, 2010. 11(1):76-91. 
25. Larsen TM, Dalskov SM, van Baak M, Jebb SA, Papadaki A, Pfeiffer AF et al. Diets with high or low protein content and glycemic index for weight-loss maintenance. N Engl J Med, 2010. 363(22):210213.

26. Wang P, Holst C, Astrup A, Bouwman FG, van Otterdijk S, Wodzig WK et al. Blood profiling of proteins and steroids during weight maintenance with manipulation of dietary protein level and glycaemic index. Br J Nutr, 2012. 107(1):106-19.

27. Benjamini Y, Hochberg Y. Controlling the false discovery rate: a practical and powerful approach to multiple testing. Journal of the Royal Statistical Society Series B (Methodological), 1995. 57(1):289 300.

28. Sole X, Guino E, Valls J, Iniesta R, Moreno V. SNPStats: a web tool for the analysis of association studies. Bioinformatics, 2006. 22(15):1928-9.

29. Bolton K, Segal D, McMillan J, Sanigorski A, Collier G, Walder K. Identification of secreted proteins associated with obesity and type 2 diabetes in Psammomys obesus. Int J Obes (Lond), 2009. 33(10):1153-65.

30. Norris RA, Damon B, Mironov V, Kasyanov V, Ramamurthi A, Moreno-Rodriguez R et al. Periostin regulates collagen fibrillogenesis and the biomechanical properties of connective tissues. J Cell Biochem, 2007. 101(3):695-711.

31. De Luca M, Chambers MM, Casazza K, Lok KH, Hunter GR, Gower BA, Fernandez JR. Genetic variation in a member of the laminin gene family affects variation in body composition in Drosophila and humans. BMC Genet, 2008. 9:52.

32. Mariman EC, Bouwman FG, Aller EE, van Baak MA, Wang P. High frequency of rare variants with a moderate-to-high predicted biological effect in protocadherin genes of extremely obese. Genes Nutr, 2014. 9(3):399.

33. Hagg P, Vaisanen T, Tuomisto A, Rehn M, Tu H, Huhtala P, Eskelinen S, Pihlajaniemi T. Type XIII collagen: a novel cell adhesion component present in a range of cell-matrix adhesions and in the intercalated discs between cardiac muscle cells. Matrix Biol, 2001. 19(8):727-42.

34. Koch M, Veit G, Stricker S, Bhatt P, Kutsch S, Zhou P et al. Expression of type XXIII collagen mRNA and protein. J Biol Chem, 2006. 281(30):21546-57.

35. Snellman A, Tu H, Vaisanen T, Kvist AP, Huhtala P, Pihlajaniemi T. A short sequence in the N-terminal region is required for the trimerization of type XIII collagen and is conserved in other collagenous transmembrane proteins. Embo J, 2000. 19(19):5051-9.

36. Peltonen S, Hentula M, Hagg P, Yla-Outinen H, Tuukkanen J, Lakkakorpi J, Rehn M, Pihlajaniemi T, Peltonen J. A novel component of epidermal cell-matrix and cell-cell contacts: transmembrane protein type XIII collagen. J Invest Dermatol, 1999. 113(4):635-42.

37. Heikkinen A, Tu H, Pihlajaniemi T. Collagen XIII: a type II transmembrane protein with relevance to musculoskeletal tissues, microvessels and inflammation. Int J Biochem Cell Biol, 2012. 44(5):714-7.

38. Kong LC, Wuillemin PH, Bastard JP, Sokolovska N, Gougis S, Fellahi S et al. Insulin resistance and inflammation predict kinetic body weight changes in response to dietary weight loss and maintenance in overweight and obese subjects by using a Bayesian network approach. Am J Clin Nutr, 2013. 98(6):1385-94.

39. Veit G, Zwolanek D, Eckes B, Niland S, Kapyla J, Zweers MC et al. Collagen XXIII, novel ligand for integrin alpha2beta1 in the epidermis. J Biol Chem, 2011. 286(31):27804-13.

40. Schluterman MK, Chapman SL, Korpanty G, Ozumi K, Fukai T, Yanagisawa H, Brekken RA. Loss of fibulin-5 binding to beta1 integrins inhibits tumor growth by increasing the level of ROS. Dis Model Mech, 2010. 3(5-6):333-42.

41. To WS, Midwood KS. Plasma and cellular fibronectin: distinct and independent functions during tissue repair. Fibrogenesis Tissue Repair, 2011. 4:21.

42. Andersen T, Dejgaard A, Astrup A, Gluud C. Increased plasma fibronectin concentrations in obesity: normalization during weight loss. Acta Med Scand, 1987. 222(3):275-9.

43. Antras J, Hilliou F, Redziniak G, Pairault J. Decreased biosynthesis of actin and cellular fibronectin during adipose conversion of 3T3-F442A cells. Reorganization of the cytoarchitecture and extracellular matrix fibronectin. Biol Cell, 1989. 66(3):247-54.

44. Kamiya S, Kato R, Wakabayashi M, Tohyama T, Enami I, Ueki M et al. Fibronectin peptides derived from two distinct regions stimulate adipocyte differentiation by preventing fibronectin matrix assembly. Biochemistry, 2002. 41(9):3270-7. 
45. Rossmeislova L, Malisova L, Kracmerova J, Tencerova M, Kovacova Z, Koc M, Siklova-Vitkova M, Viquerie N, Langin D, Stich V. Weight loss improves the adipogenic capacity of human preadipocytes and modulates their secretory profile. Diabetes, 2013. 62(6):1990-5. 
Chapter 2

Supplementary Figures and Tables 
Supplement Table 1: List of 124 candidate genes related to extracellular matrix.

\begin{tabular}{|c|c|c|c|c|c|c|}
\hline Gene ID & Gene name & $\begin{array}{l}\text { Number } \\
\text { dbSNPs }\end{array}$ & $\begin{array}{l}\text { Number } \\
\text { genotyped SNPs }\end{array}$ & Chromosome & Start bp & End bp \\
\hline ACAN & Agrecan 1 & 1750 & 33 & 15 & 89346674 & 89418585 \\
\hline ADAM11 & ADAM metallopeptidase domain 11 & 572 & 6 & 17 & 42836399 & 42859214 \\
\hline ADAM12 & ADAM metallopeptidase domain 12 & 6981 & 167 & 10 & 127700950 & 128077024 \\
\hline ADAM15 & ADAM metallopeptidase domain 15 & 532 & 9 & 1 & 155023042 & 155035252 \\
\hline ADAM17 & ADAM metallopeptidase domain 17 & 1278 & 11 & 2 & 9628615 & 9695921 \\
\hline ADAM19 & ADAM metallopeptidase domain 19 & 3290 & 43 & 5 & 156822542 & 157002783 \\
\hline ADAM22 & ADAM metallopeptidase domain 22 & 3882 & 43 & 7 & 87563458 & 87832204 \\
\hline ADAMTS1 & $\begin{array}{l}\text { A desintegrin and metalloproteinase with } \\
\text { thrombospondin type } 1 \text { motif } 1\end{array}$ & 509 & 15 & 21 & 28208606 & 28217728 \\
\hline ADAMTS4 & $\begin{array}{l}\text { A desintegrin and metalloproteinase with } \\
\text { thrombospondin type } 1 \text { motif } 4\end{array}$ & 489 & 4 & 1 & 161159538 & 161168846 \\
\hline ADAMTS5 & $\begin{array}{l}\text { A desintegrin and metalloproteinase with } \\
\text { thrombospondin type } 1 \text { motif } 5\end{array}$ & 1147 & 19 & 21 & 28290231 & 28338832 \\
\hline AZGP1 & Alpha-2-glycoprotein 1, zinc & 489 & 3 & 7 & 99564343 & 99573780 \\
\hline BGN & Biglycan & 463 & 8 & $\mathrm{x}$ & 152760397 & 152775012 \\
\hline BMP1 & Bone morphogenic protein 1 & 1366 & 15 & 8 & 22022249 & 22069839 \\
\hline CALR & Calreticulin & 498 & 4 & 19 & 13049392 & 13055303 \\
\hline CCDC80 & Coiled coil domain containing protein 80 & 1058 & 17 & 3 & 112323407 & 112368377 \\
\hline CHI3L1 & Chitinase-3-like protein 1 & 565 & 13 & 1 & 203148059 & 203155877 \\
\hline CILP & Cartilage intermediate layer protein & 653 & 9 & 15 & 65488337 & 65503826 \\
\hline CNTNAP1 & Contactin associated protein 1 & 554 & 2 & 17 & 40834631 & 40851832 \\
\hline COL11A1 & Collagen a $1(\mathrm{XI})$ chain & 4295 & 53 & 1 & 103342023 & 103574052 \\
\hline COL12A1 & Collagen a 1 (XII) chain & 2055 & 22 & 6 & 75794042 & 75915767 \\
\hline COL14A1 & Collagen a $1(\mathrm{XIV})$ chain (undulin) & 4927 & 69 & 8 & 121072019 & 121384275 \\
\hline COL15A1 & Collagen a $1(X V)$ chain & 2501 & 39 & 9 & 101705461 & 101833069 \\
\hline COL18A1 & Collagen a 1 (XVIII) chain & 2951 & 37 & 21 & 46825052 & 46933634 \\
\hline COL1A1 & Collagen a $1(I)$ chain & 1111 & 6 & 17 & 48260650 & 48278993 \\
\hline COL1A2 & Collagen a $2(I)$ chain & 1257 & 25 & 7 & 94023873 & 94060544 \\
\hline COL23A1 & Collagen a 1 (XXIII) chain & 7017 & 135 & 5 & 177664619 & 178017556 \\
\hline COL2A1 & Collagen a 1 (II) chain & 1089 & 25 & 12 & 48366748 & 48398269 \\
\hline COL3A1 & Collagen a 1 (IIII) chain & 975 & 20 & 2 & 189839046 & 189877472 \\
\hline COL4A1 & Collagen a $1(\mathrm{IV})$ chain & 3541 & 89 & 13 & 110801318 & 110959496 \\
\hline COL4A2 & Collagen a 2(IV) chain & 4826 & 84 & 13 & 110958159 & 111165374 \\
\hline COL4A3 & Collagen a $3(\mathrm{IV})$ chain & 2897 & 67 & 2 & 228029281 & 228179508 \\
\hline COL4A5 & Collagen a $5(\mathrm{IV})$ chain & 2927 & 6 & $\mathrm{x}$ & 107683074 & 107940775 \\
\hline COL5A1 & Collagen a $1(\mathrm{~V})$ chain & 4749 & 79 & 9 & 137533620 & 137736686 \\
\hline COL5A2 & Collagen a $2(\mathrm{~V})$ chain & 2410 & 10 & 2 & 189896622 & 190044605 \\
\hline COL5A3 & Collagen a $3(\mathrm{~V})$ chain & 1512 & 17 & 19 & 10070237 & 10121147 \\
\hline COL6A1 & Collagen a $1(\mathrm{VI})$ chain & 1091 & 6 & 21 & 47401651 & 47424964 \\
\hline COL6A2 & Collagen a 2(VI) chain & 1596 & 17 & 21 & 47518011 & 47552763 \\
\hline COL6A3 & Collagen a $3(\mathrm{VI})$ chain & 2292 & 42 & 2 & 238232646 & 238323018 \\
\hline CSPG4 & Chondroitin sulfate proteoglycan 4 & 895 & 5 & 15 & 75966663 & 76005189 \\
\hline CTGF & Connective tissue growth factor & 321 & 7 & 6 & 132269316 & 132272513 \\
\hline DAG1 & Dystroglycan 1 & 1125 & 6 & 3 & 49506146 & 49573048 \\
\hline DCN & Decorin (bone proteoglycan II) & 837 & 10 & 12 & 91539025 & 91576900 \\
\hline DPT & Dermatopontin & 1056 & 23 & 1 & 168664697 & 168698502 \\
\hline ECM1 & Extracellular matrix protein 1 & 439 & 2 & 1 & 150480538 & 150486265 \\
\hline ECM2 & extracellular matrix protein 2 & 828 & 6 & 9 & 95256365 & 95298937 \\
\hline EFEMP1 & $\begin{array}{l}\text { Fibulin-3 (EGF-containing fibulin-like } \\
\text { extracellular matrix protein 1) }\end{array}$ & 1133 & 15 & 2 & 56093102 & 56151274 \\
\hline EFEMP2 & $\begin{array}{l}\text { Fibulin-4 (EGF-containing fibulin-like } \\
\text { extracellular matrix protein 2) }\end{array}$ & 543 & 2 & 11 & 65633912 & 65641063 \\
\hline ELN & Elastin & 885 & 10 & 7 & 73442119 & 73484237 \\
\hline EMILIN1 & $\begin{array}{l}\text { Elastin microfibril interface-located } \\
\text { protein } 1\end{array}$ & 452 & 2 & 2 & 27301435 & 27309271 \\
\hline EMILIN2 & $\begin{array}{l}\text { Elastin microfibril interface-located } \\
\text { protein } 2\end{array}$ & 1738 & 26 & 18 & 2847028 & 2915991 \\
\hline FBLN1 & Fibulin-1 & 2283 & 50 & 22 & 45898118 & 45997015 \\
\hline FBLN2 & Fibulin-2 & 2283 & 42 & 3 & 13573824 & 13679922 \\
\hline FBLN5 & Fibulin-5 & 1620 & 42 & 14 & 92335756 & 92414331 \\
\hline FBN1 & Fibrillin 1 & 3921 & 33 & 15 & 48700503 & 48938046 \\
\hline FKBP9 & $\begin{array}{l}\text { Peptidyl-prolyl cisĐtrans isomerase } \\
\text { (FK506-binding protein 9) }\end{array}$ & 1160 & 3 & 7 & 32997017 & 33046543 \\
\hline FMOD & Fibromodulin & 606 & 8 & 1 & 203309753 & 203320617 \\
\hline FN1 & Fibronectin & 1616 & 16 & 2 & 216225163 & 216300895 \\
\hline GPC1 & Glypican 1 & 960 & 14 & 2 & 241375088 & 241407493 \\
\hline
\end{tabular}




\begin{tabular}{|c|c|c|c|c|c|c|}
\hline ITGAM & $\begin{array}{l}\text { Integrin, alpha } \mathrm{M} \text { (complement } \\
\text { component } 3 \text { receptor } 3 \text { subunit) }\end{array}$ & 1271 & 10 & 16 & 31271311 & 31344213 \\
\hline ITGAV & $\begin{array}{l}\text { Integrin, alpha } \mathrm{V} \text { (vitronectin receptor, } \\
\text { alpha polypeptide antigen } \mathrm{CD} 51 \text { ) }\end{array}$ & 1975 & 14 & 2 & 187454792 & 187545628 \\
\hline ITGB1 & $\begin{array}{l}\text { Integrin, beta } 1 \text { (fibronectin receptor, beta } \\
\text { polypeptide antigen } C D 29 \text { ) }\end{array}$ & 1998 & 23 & 10 & 33189247 & 33294720 \\
\hline ITGB2 & $\begin{array}{l}\text { Integrin, beta } 2 \text { (complement component } \\
3 \text { receptor } 3 \text { and } 4 \text { subunit) }\end{array}$ & 1409 & 29 & 21 & 46305868 & 46351904 \\
\hline ITGB5 & Integrin, beta 5 & 2300 & 42 & 3 & 124480795 & 124606674 \\
\hline LAMA2 & Laminin a- 2 chain & 9937 & 125 & 6 & 129204342 & 129837714 \\
\hline LAMA4 & Laminin a-4 chain & 2667 & 53 & 6 & 112429963 & 112576141 \\
\hline LAMB1 & Laminin b-1 chain & 1809 & 25 & 7 & 107564244 & 107643700 \\
\hline LAMB2 & Laminin $b-2$ chain & 532 & 3 & 3 & 49158547 & 49170551 \\
\hline LAMC1 & Laminin c-1 chain & 2218 & 18 & 1 & 182992595 & 183114727 \\
\hline LGALS1 & Galectin-1 & 403 & 5 & 22 & 38071615 & 38075813 \\
\hline LGALS3BP & $\begin{array}{l}\text { Lectin galactoside-binding soluble } 3 \text { - } \\
\text { binding protein }\end{array}$ & 561 & 9 & 17 & 76967320 & 76976191 \\
\hline LOX & Protein-lysine 6-oxidase & 540 & 6 & 5 & 121398890 & 121413980 \\
\hline LOXL1 & Lysyl-oxidase homologue 1 & 723 & 16 & 15 & 74218330 & 74244478 \\
\hline LUM & Lumican & 500 & 5 & 12 & 91496406 & 91505608 \\
\hline MATN2 & Matrilin-2 & 2763 & 71 & 8 & 98881068 & 99048944 \\
\hline MATN3 & Matrilin-3 & 548 & 6 & 2 & 20191872 & 20212455 \\
\hline MATN4 & Matrilin-4 & 725 & 9 & 20 & 43922085 & 43937169 \\
\hline MFAP4 & Microfibril-associated glycoprotein 4 & 359 & 2 & 17 & 19286755 & 19290553 \\
\hline MMP1 & Matrix metalloproteinase-1 & 549 & 11 & 11 & 102660651 & 102668891 \\
\hline MMP10 & Matrix metalloproteinase-10 & 579 & 15 & 11 & 102641234 & 102651359 \\
\hline MMP14 & $\begin{array}{l}\text { Matrix metalloproteinase } 14 \text {; membrane- } \\
\text { type-1 matrix metalloproteinase }\end{array}$ & 614 & 10 & 14 & 23305766 & 23318236 \\
\hline MMP19 & $\begin{array}{l}\text { Matrix metalloproteinase } 14 \text {; membrane- } \\
\text { type-1 matrix metalloproteinase }\end{array}$ & 464 & 1 & 12 & 56229217 & 56236750 \\
\hline MMP2 & Matrix metalloproteinase-2 & 2624 & 55 & 16 & 55423612 & 55540603 \\
\hline MMP9 & Matrix metalloproteinase- 9 & 685 & 6 & 20 & 44637547 & 44645200 \\
\hline NID1 & Nidogen 1 (entactin) & 1900 & 28 & 1 & 236139130 & 236228462 \\
\hline NID2 & Nidogen 2 (osteonidogen) & 1766 & 43 & 14 & 52471521 & 52535712 \\
\hline NPNT & Nephronectin & 1984 & 21 & 4 & 106815932 & 106925184 \\
\hline OGN & Mimecan (osteoglycin) & 517 & 2 & 9 & 95146249 & 95166978 \\
\hline P4HA1 & Prolyl 4-hydroxylase, alpha subunit & 1713 & 5 & 10 & 74766975 & 74856732 \\
\hline $\mathrm{P} 4 \mathrm{HB}$ & Protein-disulfide isomerise & 824 & 1 & 17 & 79801035 & 79818570 \\
\hline PCOLCE & $\begin{array}{l}\text { Procollagen C-proteinase enhancer } \\
\text { protein }\end{array}$ & 424 & 2 & 7 & 100199800 & 100205798 \\
\hline PDIA3 & Protein-disulfide isomerase A3 & 690 & 2 & 15 & 44038590 & 44065477 \\
\hline PDIA4 & Protein-disulfide isomerase $A 4$ & 877 & 6 & 7 & 148700154 & 148725733 \\
\hline PDIA6 & Protein-disulfide isomerase $A 6$ & 1696 & 23 & 2 & 10923517 & 10978103 \\
\hline PLOD1 & $\begin{array}{l}\text { Procollagen-lysine,2-oxoglutarate } 5 \text { - } \\
\text { dioxygenase } 1\end{array}$ & 1131 & 8 & 1 & 11994262 & 12035595 \\
\hline PLOD2 & $\begin{array}{l}\text { Procollagen-lysine,2-oxoglutarate 5- } \\
\text { dioxygenase } 2\end{array}$ & 1653 & 13 & 3 & 145787227 & 145881440 \\
\hline PLOD3 & $\begin{array}{l}\text { Procollagen-lysine,2-oxoglutarate 5- } \\
\text { dioxygenase } 3\end{array}$ & 678 & 6 & 7 & 100849258 & 100861701 \\
\hline POSTN & Periostin & 909 & 11 & 13 & 38136720 & 38172981 \\
\hline PPIA & $\begin{array}{l}\text { Peptidyl-prolyl cisĐtrans isomerase A } \\
\text { (cyclophilin A) }\end{array}$ & 720 & 1 & 7 & 44836279 & 44864163 \\
\hline PPIB & $\begin{array}{l}\text { Peptidyl-prolyl cisĐtrans isomerase B } \\
\text { (cyclophilin B) }\end{array}$ & 414 & 2 & 15 & 64448011 & 64455404 \\
\hline PRELP & $\begin{array}{l}\text { Proline arginine rich end leucine-rich } \\
\text { repeat protein }\end{array}$ & 689 & 0 & 1 & 203444956 & 203460480 \\
\hline PRG4 & Proteoglycan 4 & 682 & 8 & 1 & 186265405 & 186283694 \\
\hline PRSS3P1 & Trypsinogen B & 545 & 0 & 7 & 142468264 & 142471794 \\
\hline SERPINA12 & $\begin{array}{l}\text { Visceral adipose tissue-derived serine } \\
\text { protease inhibitor (vaspin) }\end{array}$ & 993 & 27 & 14 & 94953611 & 94984181 \\
\hline SERPINE1 & Plasminogen activator inhibitor 1 & 593 & 7 & 7 & 100770370 & 100782547 \\
\hline SERPINH1 & Colligin (collagen-binding protein) & 528 & 10 & 11 & 75273101 & 75283828 \\
\hline SPARC & SPARC (osteonectin) & 730 & 15 & 5 & 151040657 & 151066726 \\
\hline SPARCL1 & SPARC-like protein 1 & 1206 & 22 & 4 & 88394487 & 88452213 \\
\hline SPON1 & Spondin-1 (F-spondin) & 4807 & 75 & 11 & 13983914 & 14289646 \\
\hline SPON2 & Spondin-2 (mindin) & 1012 & 10 & 4 & 1160720 & 1202750 \\
\hline TGFBI & $\begin{array}{l}\text { Transforming growth factor-b-induced } \\
\text { protein IG-H3 }\end{array}$ & 905 & 12 & 5 & 135364584 & 135399507 \\
\hline TGFBR3 & $\begin{array}{l}\text { Betaglycan (transforming growth factor } \\
\text { beta receptor III) }\end{array}$ & 3798 & 80 & 1 & 92145902 & 92371892 \\
\hline THBS1 & Thrombospondin-1 & 654 & 9 & 15 & 39873280 & 39891667 \\
\hline
\end{tabular}




\begin{tabular}{|c|c|c|c|c|c|c|}
\hline THBS2 & Thrombospondin-2 & 1244 & 18 & 6 & 169615875 & 169654139 \\
\hline THBS3 & Thrombospondin-3 & 492 & 2 & 1 & 155165379 & 155178842 \\
\hline TIMP1 & TIMP metallopeptidase inhibitor 1 & 269 & 3 & $x$ & 47441712 & 47446188 \\
\hline TIMP2 & TIMP metallopeptidase inhibitor 2 & 1731 & 22 & 17 & 76849059 & 76921469 \\
\hline TIMP3 & TIMP metallopeptidase inhibitor 3 & 1298 & 35 & 22 & 33197687 & 33259030 \\
\hline TIMP4 & TIMP metallopeptidase inhibitor 4 & 389 & 4 & 3 & 12194551 & 12200851 \\
\hline TLL1 & Tolloid-like 1 & 4402 & 45 & 4 & 166794410 & 167025047 \\
\hline TLL2 & Tolloid-like 2 & 2629 & 49 & 10 & 98124363 & 98273675 \\
\hline TNC & Tenascin-C & 2085 & 47 & 9 & 117782806 & 117880536 \\
\hline TNN & Tenascin- $\mathrm{N}$ & 1826 & 20 & 1 & 175036994 & 175117202 \\
\hline TNXB & Tenascin- $\mathrm{X}$ & 1307 & 17 & 6 & 32008931 & 32083111 \\
\hline VCAN & Versican core protein & 2136 & 43 & 5 & 82767284 & 82878122 \\
\hline
\end{tabular}

SNPs, single nucleotide polymorphism; dbSNPs, SNPs found in database; bp, base pair. 
Chapter 2

Supplement Table 2: List of 2903 single nucleotide polymorphisms for which genotype data was retrieved.

This table can be accessed via this link:

http://link.springer.com/article/10.1007\%2Fs12263-015-0506-y\#SupplementaryMaterial 


\section{Chapter 3}

\section{A role for leukocyte and ECM remodelling of adipose tissue in the risk of weight regain after weight loss}

Nadia J.T. Roumans, Roel G. Vink, Parastoo Fazeldadeh, Marleen A. van Baak and Edwin C.M. Mariman

American Journal of Clinical Nutrition, 2017, 105 (5): 1054-1062 


\section{Abstract}

Background: Weight loss is often followed by weight regain after an energy-restricted dietary intervention (DI). When people are on diet, the volume of an adipocyte decreases by loss of triglycerides creating stress between the cell contents and the surrounding extracellular matrix (ECM). Previously, we observed that genetic variation of ECM genes is associated with an increased risk of weight regain.

Objective: We investigated the relation between expression of ECM genes during weight loss and a period of weight stabilization, and the risk of weight regain.

Design: In this randomized controlled trial, sixty-one healthy overweight/obese participants followed either a 5-week very-low-calorie diet $(500 \mathrm{kcal} / \mathrm{d})$ or a 12-week low-calorie diet $(1250 \mathrm{kcal} / \mathrm{d})$ (WL period) with subsequent a 4-week weight stable diet (WS period), and a 9-month follow-up. The WL and WS period taken together was named the DI. Abdominal subcutaneous adipose tissue biopsies were collected for microarray analysis. Gene expression changes for a broad set of ECM-related genes were correlated to the weight regain percentage.

Results: 26 of the 277 genes correlated significantly with the weight regain percentage during WL, WS or DI. Most correlations were observed in the VLCD group during WS. Four genes code for leukocyte specific receptors. These and other genes belong to a group of 26 genes of which the expression changes correlated highly $(r \geq 0.7, \mathrm{P} \leq 0.001)$ among each other. This group could be divided into three subclusters linking to two biological processes: leukocyte integrin gene activity and ECM remodelling, whereas a link with insulin sensitivity is apparent.

Conclusions: Our present findings indicate the importance of adipose tissue leukocytes for the risk of weight regain. ECM modification seems also involved and we observed a link with insulin sensitivity. 


\section{Introduction}

Overweight and obesity are growing public health concerns worldwide due to the increased risk for the metabolic syndrome and the development of type 2 diabetes, cardiovascular diseases and cancer $(1,2)$. Weight loss by an energy-restricted dietary intervention, increased physical activity, pharmacological and/or surgical treatment reduces the disease risk and produces positive health outcomes in overweight and obese people $(3,4)$. However, long-term weight loss maintenance has been proven to be difficult $(5,6)$. Generally, up to $80 \%$ of the people are unsuccessful in maintaining weight loss $(7,8)$. Therefore, it is crucial to gain more knowledge about the mechanisms that influence the risk for weight regain. There is now substantial evidence that the adipose tissue is one of the important determinants in the process of weight regain (9). Mariman et al. suggested that cellular stress, accumulated in adipocytes during a negative energy balance, is a driving force behind the risk for weight regain (10). When people are on an energy-restricted diet, the volume of an adipocyte decreases by loss of triglycerides creating stress between the cell contents and the surrounding extracellular matrix (ECM) (11). Indeed, we have been able to show that on average people who regain weight, have higher levels of stress proteins in adipose tissue than those who succeed in maintaining the lost weight (12). Furthermore, we have demonstrated that certain genetic variations of ECM genes are associated with an increased risk for weight regain (13). In the present study we aimed to further investigate the influence of the ECM on weight regain.

Weight loss induces changes in the ECM not only by cellular stress, but also in other ways. It is generally observed that the development of obesity is accompanied by an onset of low grade inflammation. This involves a chemotactic attraction of cells of the innate immune system into the adipose tissue leading to a situation in which pro-inflammatory immune cells outbalance the antiinflammatory cells (14). Such a state of increased inflammation may have systemic consequences as it may promote the development of insulin resistance. Weight loss usually reduces the inflammatory activity of the adipose tissue, which may not be directly obvious during energy restriction. Studies have shown that expression of genes involved in inflammation and innate immunity was increased (15) or unchanged (16) in human adipose tissue directly after short-term weight loss, but was downregulated during a subsequent weight stabilization period (WS period) (15-17). Diapedesis of leukocytes during weight gain and possible emigration of leukocytes after weight loss, require remodeling of the ECM. Therefore, this change in content of immune cells may influence the risk for weight regain. Here, we have analysed gene expression of a large number of ECM genes during weight loss and weight stabilization in relation to weight regain.

\section{Materials and Methods}

\section{Participants and study design}

Sixty-one overweight and obese (BMI $27-36 \mathrm{~kg} / \mathrm{m}^{2}$ ) Caucasian participants underwent an energyrestricted dietary intervention (Figure 1). The whole study design has been described in detail previously (18). In short, individuals were recruited by advertisement via local media. Exclusion criteria were smoking, cardiovascular disease, type 2 diabetes mellitus, liver or kidney disease, use of medication that influences body weight regulation, pregnancy, marked alcohol consumption (>21 alcoholic units per week for men and $>14$ alcoholic units per week for women), elevated fasting glucose $(>6.1 \mathrm{mmol} / \mathrm{L})$, total cholesterol $(>7.0 \mathrm{mmol} / \mathrm{L})$ or triacylglycerol $(>3.0 \mathrm{mmol} / \mathrm{L})$ concentrations, or blood pressure $(>160 / 100 \mathrm{mmHg})$. Participants were randomly assigned to either 
a very-low-calorie diet (VLCD, rapid weight loss) or a low-calorie diet (LCD, slow weight loss) group. Participants in the VLCD group underwent a 5 -week diet with about $500 \mathrm{kcal} /$ day by consuming three meal replacements per day (Modifast; Nutrition et Santé Benelux, Breda, The Netherlands). Participants in the LCD group underwent a 12-week diet with about $1250 \mathrm{kcal} /$ day, designed by a dietician. Both groups were targeted to lose approximately $10 \%$ body weight during this weight loss period (WL, T1-T2). Following WL, all participants underwent a 4-week weight maintenance diet based on their individual energy requirements. This weight stable period (WS, T2-T3) was designed to investigate the effect of weight loss of approximately $10 \%$, without the interfering effect of a pronounced negative energy balance. The WL and WS period taken together was named the dietary intervention (DI, T1-T3). The study dietician provided dietary advice according to the Dutch national guidelines (19) to both groups, to assist in remaining weight stable throughout the WS period and to assist in weight loss during the WL period in the LCD group. After the WS period, participant's body weight was monitored for 9 months (follow-up, T3-T4) by monthly meetings with a dietician. During this follow-up, participants did not receive advice on monitoring and limiting food intake to mimic non-restricted free-living conditions.

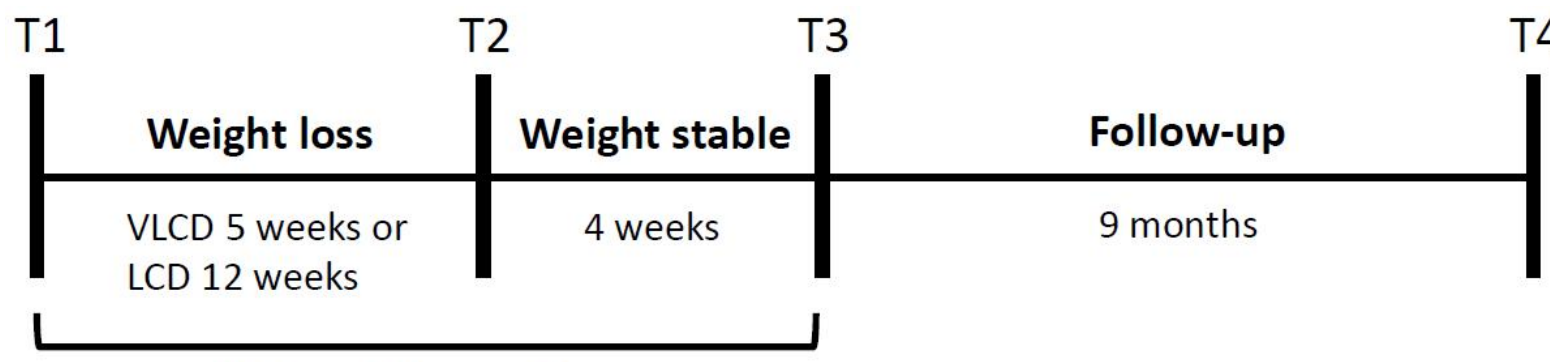

\section{Dietary intervention}

Figure 1: Schematic overview of the study design. Measurements were performed at the start of the study (T1), at the end of the weight loss period (T2), weight stable period (T3), and follow-up (T4). The dietary intervention period (DI) is the weight loss period and weight stable period taken together. LCD, low calorie-diet $(n=27)$; VLCD, very-low-calorie diet $(n=26)$.

At the start of the study (T1) and at the end of each period (T2, T3 and T4) abdominal subcutaneous adipose tissue biopsies were collected, body composition was determined and body weight, height, blood pressure, hip and waist circumference were measured after overnight fasting. Body volume was determined with air-displacement plethysmography using a Bod Pod device (Cosmed, Italy, Rome) according to the manufacturer's instructions and as described by Dempster et al. (20). Body composition was calculated from body density according to the two-compartment model by Siri (21). During the dietary intervention (T1-T3), four participants withdrew from the study, in three participants we could not collect enough biopsy material and in one participant the gene expression results deviated strongly from the others and were therefore excluded. Characteristics of the remaining fifty-three participants at $\mathrm{T} 1, \mathrm{~T} 2, \mathrm{~T} 3$ and $\mathrm{T} 4$ are displayed in Table 1.

This study was conducted according to the Declaration of Helsinki guidelines and registered on ClinicalTrials.gov (registration number: NCT01559415). All procedures involving human participants were approved by the Central Committee on Human Research and by the Medical Ethical Committee of Maastricht University, The Netherlands. Written informed consent was obtained from all participants. 


\section{Adipose tissue biopsy}

Abdominal subcutaneous adipose tissue biopsies were obtained by needle biopsy under local anaesthesia (2\% lidocaine, Fresenius Kabi, Zeist, the Netherlands) after an overnight fast at T1, T2 T3, and T4. Tissue was immediately rinsed in sterile saline, frozen in liquid nitrogen and stored at $-80^{\circ} \mathrm{C}$ until RNA isolation.

\section{Adipose tissue RNA isolation and microarray analysis}

For RNA isolation, adipose tissue samples from T1, T2 and T3 were used. Total RNA was extracted from approx. $150 \mathrm{mg}$ frozen adipose tissue using TRIzol reagent (Invitrogen, Breda, The Netherlands). Total RNA (100 ng per sample) was labeled by Whole-Transcript Sense Target Assay and hybridized to human whole-genome Affymetrix Gene 1.1 ST arrays targeting 19654 unique genes (Affymetrix, Santa Clara, CA, USA). Sample labeling, hybridization to chips and image scanning was performed according to manufacturer's instructions. Microarray signals were normalized using robust multichip average (RMA). Genes with normalized signals $>20$ on at least 15 arrays were defined as expressed (11532 genes). Gene expression changes within groups during a certain period (WL period, WS period or DI period) were defined as significantly different when the q-value was $<0.05$ in a paired t-test with Bayesian correction (Limma) (22). Array data have been submitted to the Gene Expression Omnibus (number GSE77962).

\section{Selection of ECM genes}

In this study, 277 genes related to the extracellular matrix were selected for the analysis based on previously measured ECM genes (13) and by selecting for the term "extracellular matrix" in the Gene Ontology Biological Process (GO-BP) description (Supplement Table 1).

\section{Calculations}

In this study, we wanted to analyse the relation between gene expression changes and weight regain. As a value for weight regain, we calculated the weight regain percentage (WR\%) during follow-up as follows: ((weight at T4 - weight at T3) $\div$ weight at T3) x 100\%. For gene expression change we calculated the fold change (FC) of gene expression during WL, WS or DI. Gene expression results from the microarray were expressed as $\log _{2}$ transformed values. Gene expression changes during certain periods were calculated as follows: WL $\left(\log _{2} T 2-\log _{2} T 1\right)$, WS $\left(\log _{2} T 3-\log _{2}\right.$ T2) and DI $\left(\log _{2}\right.$ T3 $-\log _{2}$ T1). These $\log _{2}$ ratios $(L R)$ during $W L, W S$ and $D I$ were transformed into fold changes $\left(F C=2^{L R}\right.$ if $L R \geq 0$ and $F C=(-1) 2^{-L R}$ if $\left.L R<0\right)$.

\section{Clustering}

First, we performed Pearson correlations between the WR\% and the changes in expression of the 277 ECM genes during the VLCD WL and WS phase. The ECM genes that correlated significantly $(\mathrm{P} \leq 0.05)$ with the $W R \%$ were clustered based on their correlation among each other $(r>0.6, \mathrm{P}<0.002)$. For a second analysis, we performed an extended clustering in which the changes of the 277 ECM gene expressions were correlated with each other during the weight stabilisation phase in the VLCD. Next, genes were selected that significantly correlated $(r \geq 0.7$ and $P \leq 0.001)$. With those genes a correlation matrix was constructed but to further minimize the chance of including false positives and focus on closely related genes, a confined correlation cluster was constructed by removing genes with less than 5 significant correlations to other genes in the cluster. The clusters were constructed to provide possible functional or regulatory information. 


\section{Statistical analysis}

Data are presented as mean \pm SEM. Comparisons of variables within a group were made with dependent T-test. Between-group comparisons (VLCD vs. LCD) were made with independent T-test. To determine the link between weight regain and ECM genes, Pearson R correlations were performed between the WR\% and the gene expression changes of the 277 ECM genes during WL, WS and DI. Statistical calculations were done using SPSS 20.0 for Windows (SPSS Inc, Chicago, IL). P $\leq 0.01$ was considered statistically significant unless otherwise stated. All variables were checked for normal distribution, and variables with a skewed distribution were In-transformed to satisfy conditions of normality. Extreme outliers (values higher than $3 x$ interquartile range calculated with SPSS) influencing the data were removed during statistical analyses.

\section{Results}

\section{Clinical characteristics}

Characteristics of the fifty-three participants at study start (T1), end of WL (T2), end of WS (T3) and end of follow-up (T4) are displayed in Table 1. The VLCD and LCD groups were comparable at study start for weight, BMI, body fat \%, fat free mass, and hip and waist circumference. During WL (T1-T2), participants decreased in body weight, BMI, hip and waist circumference, body fat percentage and fat free mass (Table 1). After WL, VLCD and LCD groups were still comparable for all parameters. In the subsequent 4-week weight stable period (T2-T3), hip circumference, body fat $\%$ and fat free mass changed (Table 1). Still no significant differences were observed between VLCD and LCD after the WS period. During the dietary intervention (T1-T3), all parameters significantly decreased except for fat free mass in the LCD group (Table 1). Body weight, BMI, waist circumference, body fat percentage, body fat and fat free mass increased during follow-up (T3-T4). No differences were found between VLCD and LCD for the average weight loss percentage $(-9.7 \pm 1.2 \%$ and $-8.8 \pm 2.9 \%, P=0.228)$ and WR\% $(5.4 \pm 4.5 \%$ and $5.3 \pm 3.8 \%, P=0.957)$.

\section{Changes of ECM gene RNA levels correlate with weight regain percentage}

Changes during WL, WS and DI in the expression of 277 ECM-related genes were checked for correlation $(r \geq 0.5, \mathrm{P} \leq 0.01)$ with $\mathrm{WR} \%$. Analysis was performed separately for the dietary groups and showed 17 significant correlations for the VLCD group and 9 for the LCD group (Table 2). For VLCD most correlations (11/17) were in the WS phase, whereas for LCD most were found in the DI phase (7/9). There was no overlap of correlated genes between the dietary groups. The strongest correlation was found in the LCD group with discoidin domain receptor tyrosine kinase 1 (DDR1) in the WL phase $(r=0.698, \mathrm{P}<0.001)$. DDR1 is a cell receptor for collagen and in that way is involved in cell attachment to the extracellular matrix. In endothelial cells and lung cancer cells, collagen type IV alpha 5 (COL4A5) directs the DDR1 activity (23). Here we observed a negative correlation between COL4A5 gene expression and WR\%, but in the VLCD DI phase. 
Table 1: Subject characteristics at study start, end of weight loss, end of weight stable and end of follow-up ${ }^{1}$.

\begin{tabular}{|c|c|c|c|c|c|c|c|c|}
\hline & \multicolumn{2}{|c|}{ Study start (T1) } & \multicolumn{2}{|c|}{ End of WL (T2) } & \multicolumn{2}{|c|}{ End of WS (T3) } & \multicolumn{2}{|c|}{ End of follow-up (T4) } \\
\hline & VLCD & LCD & VLCD & LCD & VLCD & LCD & VLCD & LCD \\
\hline Sex (male/female) & $12 / 14$ & $13 / 14$ & & & & & & \\
\hline Age (years) & $50.4 \pm 1.5$ & $51.7 \pm 2.1$ & & & & & & \\
\hline Weight (kg) & $92.1 \pm 1.9$ & $92.8 \pm 2.0$ & $83.1 \pm 1.6^{2}$ & $84.6 \pm 2.0^{2}$ & $82.9 \pm 1.7^{3}$ & $84.5 \pm 2.0^{3}$ & $87.7 \pm 2.0^{2}$ & $89.2 \pm 2.1^{2}$ \\
\hline $\mathrm{BMI}\left(\mathrm{kg} / \mathrm{m}^{2}\right)$ & $30.8 \pm 0.4$ & $31.5 \pm 0.5$ & $27.8 \pm 0.4^{2}$ & $28.7 \pm 0.5^{2}$ & $27.7 \pm 0.4^{3}$ & $28.7 \pm 0.5^{3}$ & $29.1 \pm 0.5^{2}$ & $30.3 \pm 0.5^{2}$ \\
\hline Hip circumference $(\mathrm{cm})$ & $111.0 \pm 1.1$ & $110.7 \pm 1.4$ & $105.0 \pm 1.0^{2}$ & $106.1 \pm 1.5^{2}$ & $104.8 \pm 1.0^{3}$ & $104.8 \pm 1.5^{2,3}$ & $105.4 \pm 1.4$ & $107.0 \pm 1.9$ \\
\hline Waist circumference $(\mathrm{cm})$ & $101.3 \pm 1.6$ & $102.5 \pm 2.1$ & $93.5 \pm 1.4^{2}$ & $95.2 \pm 1.9^{2}$ & $94.6 \pm 1.4^{3}$ & $94.4 \pm 2.1^{3}$ & $97.6 \pm 1.7^{2}$ & $98.6 \pm 2.1^{2}$ \\
\hline Body fat (\%) & $39.5 \pm 1.6$ & $40.6 \pm 1.9$ & $34.8 \pm 2.0^{2}$ & $34.7 \pm 2.2^{2}$ & $33.7 \pm 2.0^{2,3}$ & $34.1 \pm 2.3^{2,3}$ & $36.0 \pm 1.9^{2}$ & $36.9 \pm 2.2^{2}$ \\
\hline Fat free mass $(\mathrm{kg})$ & $55.7 \pm 2.4$ & $55.5 \pm 2.3$ & $54.2 \pm 2.3^{2}$ & $55.0 \pm 2.3^{2}$ & $54.9 \pm 2.4^{2,3}$ & $55.3 \pm 2.3$ & $55.9 \pm 2.4^{2}$ & $56.1 \pm 2.5$ \\
\hline
\end{tabular}

${ }^{1}$ All values are means \pm SEMs. VLCD $n=26$ and $L C D n=27$. No significant difference ( $P \leq 0.05$ ) between the VLCD and $L C D$ group at study start, end of $W L$, end of $W S$ and end of follow- 4 .

${ }^{2}$ Significant change $(\mathrm{P} \leq 0.05)$ between this time point and the previous time point with dependant $\mathrm{T}$ test.

${ }^{3}$ Significant change $(P \leq 0.05)$ between the end of WS (T3) and study start (T1) with dependant $T$ test.

$\mathrm{BMI}$, body mass index; LCD, low-calorie diet; VLCD, very-low-calorie diet. 
$\vec{\sigma}$ Table 2: Correlation coefficients between the weight regain percentage and the fold changes in ECM gene expression during the weight loss, weight stable and dietary intervention period separated based on $\operatorname{diet}^{1}$.

\begin{tabular}{|c|c|c|c|c|c|c|c|c|}
\hline \multirow[t]{2}{*}{ Gene ID } & \multirow{2}{*}{ Gene name } & \multicolumn{3}{|l|}{ VLCD } & \multicolumn{3}{|l|}{ LCD } & \multirow[t]{2}{*}{ P-value } \\
\hline & & WL & WS & DI & WL & WS & DI & \\
\hline$A B \mid 3 B P$ & ABI family, member 3 (NESH) binding protein & 0.593 & & & & & & 0.003 \\
\hline ACTN1 & Actinin, alpha 1 & & 0.661 & & & & & 0.001 \\
\hline ADAMTSL4 & ADAMTS-like 4 & & & & & -0.626 & & 0.001 \\
\hline COL2OA1 & Collagen, type XX, alpha 1 & & & & & & -0.552 & 0.008 \\
\hline COL4A5 & Collagen, type IV, alpha 5 & & & -0.568 & & & & 0.003 \\
\hline DDR1 & Discoidin domain receptor tyrosine kinase 1 & & & & 0.698 & & & $<0.001$ \\
\hline DMD & Dystrophin & 0.560 & -0.664 & & & & & $0.005 ; 0.001$ \\
\hline FBLN1 & Fibulin 1 & & & 0.577 & & & & 0.003 \\
\hline FBLN2 & Fibulin 2 & & & & & & 0.551 & 0.008 \\
\hline FZD4 & Frizzled class receptor 4 & & & & & & 0.571 & 0.006 \\
\hline ICAM3 & Intercellular adhesion molecule 3 & & 0.566 & & & & & 0.006 \\
\hline ITGA5 & Integrin, alpha 5 & & 0.591 & & & & & 0.003 \\
\hline ITGAL & Integrin, alpha L & & 0.560 & & & & & 0.005 \\
\hline ITGAM & Integrin, alpha M & & 0.620 & & & & & 0.002 \\
\hline ITGAX & Integrin, alpha $\mathrm{X}$ & & 0.614 & & & & & 0.002 \\
\hline ITGB2 & Integrin, beta 2 & & 0.571 & & & & & 0.005 \\
\hline LAMA2 & Laminin, alpha 2 & & & & & & 0.661 & 0.001 \\
\hline LAMC1 & Laminin, gamma 1 (formerly LAMB2) & & & & & & 0.568 & 0.006 \\
\hline LGALS1 & Lectin, galactoside-binding, soluble, 1 & & & 0.661 & & & & $<0.001$ \\
\hline MMP15 & Matrix metallopeptidase 15 (membrane-inserted) & & & 0.635 & & & & 0.001 \\
\hline PLOD2 & Procollagen-lysine, 2-oxoglutarate 5-dioxygenase 2 & & -0.601 & & & & & 0.002 \\
\hline PPIB & Peptidylprolyl isomerase B (cyclophilin B) & & 0.533 & & & & & 0.009 \\
\hline PXDN & Peroxidasin & & & & & & 0.563 & 0.006 \\
\hline SPARCL1 & Secreted Protein Acidic And Cysteine Rich-like 1 & & -0.561 & & & & & 0.007 \\
\hline TIMP4 & TIMP metallopeptidase inhibitor 4 & & & & & & 0.547 & 0.008 \\
\hline
\end{tabular}

${ }^{1}$ Values are significant ( $\left.\mathrm{P} \leq 0.01\right)$ Pearson R's correlation coefficients ( $r$ ) between weight regain and the fold change (FC) of gene expression during weight loss (T2-T1), weight stable (T3-T2) and dietary intervention (T3-T1). Weight regain percentage is calculated: ((weight after follow-up - weight after WS) $\div$ weight after WS) $\times 100 \%$. LCD, low-calorie diet ( $\mathrm{n}=27)$; VLCD, very-lowcalorie diet $(n=26)$. 


\section{Cluster analysis of VLCD WS correlating genes}

Eleven genes correlated with WR\% during the VLCD WS phase. We therefore tried to see if those genes could be clustered in an attempt to learn more about the underlying processes. Clustering was based on correlation $(r>0.6, \mathrm{P}<0.002)$ between expression changes of these 11 genes during the VLCD WS phase. This approach assumes that co-expressed genes may have closely related functions or take part in the same functional process. All eleven genes could be clustered (Figure 2). The genes that negatively correlate with WR\%, procollagen-lysine 2-oxoglutarate 5-dioxygenase 2 (PLOD2), dystrophin $(D M D)$ and secreted protein acidic and cysteine rich-like 1 (SPARCL1), were also negatively correlated to the other genes, whereas PLOD2 and DMD correlated positively to each other just like the other genes of the cluster.

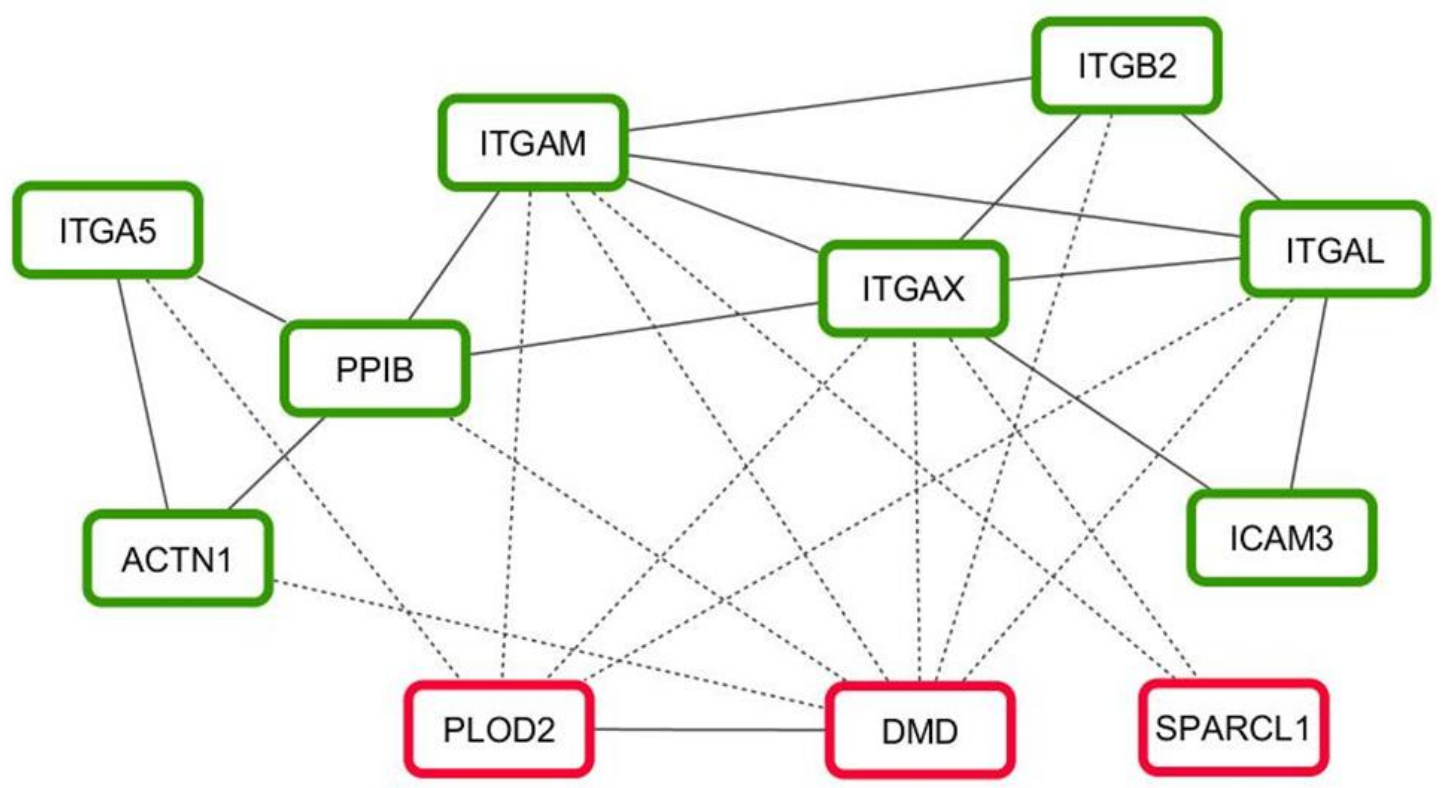

Figure 2: Correlation network between expression changes of genes during the weight stabilization phase in the VLCD $(n=26)$ group. Only Pearson R correlations with an $r>0.6, P<0.002$ are depicted in this figure. The cluster exists of genes that positively (green outline) or negatively (red outline) correlated with the weight regain percentage. Positive correlations between genes are depicted with a solid line, negative correlations with a dashed line. ACTN1, actinin, alpha 1 ; DMD, dystrophin; ICAM3, intercellular adhesion molecule 3; ITGA5, integrin, alpha 5; ITGAL, integrin, alpha L; ITGAM, integrin, alpha M; ITGAX, integrin, alpha X; ITGB2, integrin, beta 2; PLOD2, procollagen-lysine, 2-oxoglutarate 5-dioxygenase 2; PPIB, peptidylprolyl isomerase B; SPARCL1, SPARC-like 1; VLCD, very-low-calorie diet.

We then performed an extended clustering starting with the VLCD WS correlating genes to find strong correlations with other ECM-related genes $(r \geq 0.7, \mathrm{P}<0.001)$. Genes had to correlate with at least 5 other genes in the cluster in order to minimize false positives. This resulted in a cluster of 26 genes including 5 of the genes correlating with weight regain percentage: integrin alpha L (ITGAL), integrin alpha $\mathrm{M}$ (ITGAM), integrin alpha $\mathrm{X}$ (ITGAX), integrin beta 2 (ITGB2) and peptidylprolyl isomerase $B(P P I B)$. Rearranging the genes according to their strong correlation values resulted in three subclusters (Table 3), a small cluster of seven genes including the four integrin genes, and two larger overlapping clusters. All except one of the genes were positively correlated. Only the gene for fibroblast growth factor 2 (FGF2) correlated negatively to the genes of the third subcluster. The genes for matrix metallopeptidase 9 (MMP9), elastin microfibril interface 2 (EMILIN2) and calpain 1 (CAPN1) seem to connect the small cluster with the two larger clusters. The overlap becomes more evident when similar clustering is done for the VLCD WL phase (Supplement Table 2). 


\section{Gene expression changes during the WS phase in the VLCD group}

Four integrin genes are part of the small cluster, which are positively correlated with WR\%. Most of these integrins are categorized as having an important or even specific function in leukocytes (24). Table 4 shows the average change in expression of integrin genes during the VLCD WS phase. The gene for ITGB2, which forms complexes with ITGAL, ITGAM and ITGAX, was significantly downregulated. Also the genes for ITGAM and ITGAX were significantly down-regulated. In addition, the integrin alpha $E$ (ITGAE) gene, which complexes with integrin beta 7 (ITGB7) for integrin formation, was significantly down-regulated. It indicates that leukocyte-specific integrins in the adipose tissue are down-regulated during the WS phase. Since some of these genes are positively correlated to weight regain percentage, it suggests that lack of leukocyte-specific down-regulation during the weight stabilization period increases the risk of weight regain. We were unable to separately investigate the cellular composition of the adipocytes and stromal vascular fractions. In an attempt to obtain such information, we analysed for the WS phase the expression changes of genes coding for surface markers present on leukocytes using the microarray data. Our observations point to a lowering of gene expression in cells of the myeloid lineage but not in lymphoid cells (Supplement Table 3).

Table 4: Gene expression changes during the weight stabilization phase in the VLCD group ${ }^{1}$.

\begin{tabular}{|c|c|c|c|c|}
\hline Gene ID & Gene name & Remark & Fold change & Q-value \\
\hline ITGA1 & Integrin Subunit Alpha 1 & Receptor for collagen ${ }^{3}$ & 1.15 & 0.008 \\
\hline ITGA2 & Integrin Subunit Alpha 2 & Receptor for collagen ${ }^{3}$ & 1.17 & 0.003 \\
\hline ITGA10 & Integrin Subunit Alpha 10 & Receptor for collagen ${ }^{3}$ & $\mathrm{Nd}$ & nd \\
\hline ITGA11 & Integrin Subunit Alpha 11 & Receptor for collagen ${ }^{3}$ & 1.25 & $<0.001$ \\
\hline ITGA5 ${ }^{2}$ & Integrin Subunit Alpha 5 & Receptor for fibronectin ${ }^{3}$ & 1.01 & 0.458 \\
\hline ITGA3 & Integrin Subunit Alpha 3 & Receptor for laminin ${ }^{3}$ & -1.56 & 0.000 \\
\hline ITGA6 & Integrin Subunit Alpha 6 & Receptor for laminin ${ }^{3}$ & 1.19 & 0.000 \\
\hline ITGA7 & Integrin Subunit Alpha 7 & Receptor for laminin ${ }^{3}$ & 1.26 & 0.000 \\
\hline ITGA4 & Integrin Subunit Alpha 4 & Leukocyte adhesion $^{4}$ & -1.15 & 0.172 \\
\hline ITGA9 & Integrin Subunit Alpha 9 & Leukocyte adhesion $n^{5,6}$ & -1.01 & 0.493 \\
\hline ITGB2 $^{2}$ & Integrin Subunit Beta 2 & Leukocyte-specific receptor ${ }^{3}$ & -1.64 & 0.002 \\
\hline ITGAD & Integrin Subunit Alpha D & Leukocyte-specific receptor ${ }^{3}$ & -1.01 & 0.483 \\
\hline $\mathrm{ITGAL}^{2}$ & Integrin Subunit Alpha L & Leukocyte-specific receptor ${ }^{3}$ & -1.05 & 0.416 \\
\hline ITGAM $^{2}$ & Integrin Subunit Alpha M & Leukocyte-specific receptor $^{3}$ & -1.39 & 0.002 \\
\hline ITGAX ${ }^{2}$ & Integrin Subunit Alpha X & Leukocyte-specific receptor $^{3}$ & -1.54 & 0.014 \\
\hline ITGB7 & Integrin Subunit Beta 7 & Leukocyte-specific receptor $^{3}$ & 1.01 & 0.502 \\
\hline ITGAE & Integrin Subunit Alpha E & Leukocyte-specific receptor $^{3}$ & -1.33 & 0.001 \\
\hline
\end{tabular}


Table 3: Correlation matrix of changes in ECM gene expressions with each other during the weight stabilisation phase in the VLCD ${ }^{1}$.

\begin{tabular}{|c|c|c|c|c|c|c|c|c|c|c|c|c|c|c|c|c|c|c|c|c|c|c|c|c|c|c|}
\hline & \begin{tabular}{|l|} 
CTSS \\
\end{tabular} & ITGAX & ITGAM & ITGB2 & ITGAL & \begin{tabular}{|l|} 
TGFB1 \\
\end{tabular} & CHI3L1 & MMP9 & TIMP1 & TGFBI & \begin{tabular}{|l|} 
SPP1 \\
\end{tabular} & CD44 & MMP19 & EMILIN2 & CTSB & CTSD & \begin{tabular}{|l|} 
PDIA44 \\
\end{tabular} & \begin{tabular}{|l|} 
PPIB \\
\end{tabular} & ITGA3 & CAPN1 & NCSTN & \begin{tabular}{|l|l|} 
CALR \\
\end{tabular} & \begin{tabular}{|c|} 
COLGALT1 \\
\end{tabular} & PLOD1 & P4HB & FGF2 \\
\hline CTSS & 1 & \begin{tabular}{|l|l|}
0.894 \\
\end{tabular} & 0.933 & 0.945 & 0.755 & \begin{tabular}{|l|l|}
0.717 \\
\end{tabular} & 0.878 & 0.721 & 0.224 & 0.238 & \begin{tabular}{|l|}
0.340 \\
\end{tabular} & 0.285 & 0.566 & 0.753 & \begin{tabular}{|l|}
0.709 \\
\end{tabular} & \begin{tabular}{|l|}
0.691 \\
\end{tabular} & 0.472 & \begin{tabular}{|l|l|}
0.638 \\
\end{tabular} & \begin{tabular}{|l|}
0.651 \\
\end{tabular} & 0.675 & 0.587 & 0.575 & 0.512 & \begin{tabular}{|l|l|}
0.555 \\
\end{tabular} & \begin{tabular}{|l|l|}
0.466 \\
\end{tabular} & 0.510 \\
\hline ITGAX & 0.894 & 1 & 0.868 & 963 & 0.870 & \begin{tabular}{|l|l|}
0.697 \\
\end{tabular} & 596 & 700 & 420 & 381 & \begin{tabular}{|l|l|}
0.490 \\
\end{tabular} & \begin{tabular}{|l|}
0.409 \\
\end{tabular} & 657 & 226 & 649 & \begin{tabular}{|l|l|}
0.671 \\
\end{tabular} & 0.503 & \begin{tabular}{|l|l|}
0.634 \\
\end{tabular} & \begin{tabular}{|l|}
0.651 \\
\end{tabular} & 0.736 & 0.493 & \begin{tabular}{|l|}
0.601 \\
\end{tabular} & 523 & .538 & \begin{tabular}{|l|l|}
0.278 \\
\end{tabular} & 550 \\
\hline ITGAM & 33 & 868 & 1 & 903 & 775 & 72 & 302 & 532 & 290 & 325 & 375 & 273 & 39 & 67 & 679 & 516 & 0.558 & \begin{tabular}{|l|l|}
0.687 \\
\end{tabular} & \begin{tabular}{|l|}
0.710 \\
\end{tabular} & 303 & 553 & \begin{tabular}{|l|}
0.641 \\
\end{tabular} & 49 & \begin{tabular}{l|l}
592 \\
\end{tabular} & 498 & \\
\hline ITGB2 & 45 & 63 & 903 & 1 & 838 & 776 & 889 & 17 & 64 & 83 & 163 & 181 & 31 & & 558 & 565 & 405 & 562 & 534 & 69 & 16 & 001 & & 884 & 27 & \\
\hline ITGAL & 55 & 370 & 775 & .838 & 1 & 707 & 644 & 520 & 298 & 209 & 404 & 242 & 46 & & 463 & 480 & 403 & 534 & 543 & 745 & 998 & 523 & & \begin{tabular}{l|l|}
463 \\
\end{tabular} & .069 & 540 \\
\hline TGFB1 & 717 & 597 & 772 & 776 & 707 & 1 & 0.619 & 419 & 344 & 279 & 136 & \begin{tabular}{|l|}
0.207 \\
\end{tabular} & 505 & 671 & 579 & 587 & 478 & \begin{tabular}{|l|}
0.535 \\
\end{tabular} & 0.805 & 700 & .468 & 0.493 & 592 & .454 & 0.324 & 0.573 \\
\hline \begin{tabular}{|l|} 
CHI3L1 \\
\end{tabular} & 0.878 & 0.696 & 0.802 & \begin{tabular}{|l|l|}
0.889 \\
\end{tabular} & 0.544 & \begin{tabular}{|l|}
0.619 \\
\end{tabular} & 1 & 0.771 & 0.658 & 0.631 & 0.755 & 0.600 & 803 & 0.662 & 0.622 & \begin{tabular}{|l|l|}
0.677 \\
\end{tabular} & 0.523 & \begin{tabular}{|l|}
0.556 \\
\end{tabular} & 0.530 & 0.632 & \begin{tabular}{|l|}
0.480 \\
\end{tabular} & 0.582 & 463 & \begin{tabular}{|l|}
0.464 \\
\end{tabular} & \begin{tabular}{|l|}
0.536 \\
\end{tabular} & 0.490 \\
\hline \begin{tabular}{|l|} 
MMP9 \\
\end{tabular} & 0.721 & 0.700 & 0.632 & 0.617 & 0.520 & \begin{tabular}{|l|l|}
0.419 \\
\end{tabular} & 0.990 & 1 & 0.681 & 0.674 & 0.804 & 0.649 & 867 & 0.720 & 0.693 & 0.747 & 0.571 & \begin{tabular}{|l|l|}
0.595 \\
\end{tabular} & 0.569 & \begin{tabular}{|l|}
0.641 \\
\end{tabular} & 0.540 & \begin{tabular}{|l|l|}
0.628 \\
\end{tabular} & 528 & \begin{tabular}{|l|}
0.504 \\
\end{tabular} & 0.685 & 0.514 \\
\hline \begin{tabular}{|l|} 
TIMP1 \\
\end{tabular} & \begin{tabular}{|l|}
0.224 \\
\end{tabular} & \begin{tabular}{|l|l|} 
\\
\end{tabular} & 0.290 & \begin{tabular}{|l|l|}
0.164 \\
\end{tabular} & 0.298 & \begin{tabular}{|l|l|}
0.344 \\
\end{tabular} & \begin{tabular}{|l|}
0.658 \\
\end{tabular} & 681 & 1 & \begin{tabular}{|l|}
0.876 \\
\end{tabular} & \begin{tabular}{|l|l|}
0.724 \\
\end{tabular} & \begin{tabular}{|l|l|}
0.838 \\
\end{tabular} & 760 & 736 & \begin{tabular}{|l|l|}
0.706 \\
\end{tabular} & \begin{tabular}{|l|}
0.696 \\
\end{tabular} & \begin{tabular}{|l|l|}
0.723 \\
\end{tabular} & \begin{tabular}{|l|l|}
0.803 \\
\end{tabular} & \begin{tabular}{|l|l|}
0.603 \\
\end{tabular} & 0.615 & .536 & \begin{tabular}{|l|}
0.691 \\
\end{tabular} & 664 & \begin{tabular}{|l|l|}
.611 \\
\end{tabular} & \begin{tabular}{|l|l|}
0.334 \\
\end{tabular} & 0.692 \\
\hline TGFBI & 8 & 381 & 0.325 & 083 & 0.209 & 279 & 631 & 674 & 876 & 1 & .818 & \begin{tabular}{|l|}
0.778 \\
\end{tabular} & 0.804 & 68 & 0.763 & \begin{tabular}{|l|l|}
0.699 \\
\end{tabular} & 0.717 & 0.740 & 0.642 & 0.587 & 0.575 & 0.665 & 693 & .522 & 0.452 & 0.579 \\
\hline \begin{tabular}{|l|} 
SPP1 \\
\end{tabular} & 40 & 90 & 0.375 & 163 & \begin{tabular}{|l|l|}
0.404 \\
\end{tabular} & 36 & 755 & 0.804 & 24 & 318 & & \begin{tabular}{|l|l|}
0.674 \\
\end{tabular} & 332 & 15 & \begin{tabular}{|l|l|}
0.790 \\
\end{tabular} & \begin{tabular}{|l|l|}
0.754 \\
\end{tabular} & 681 & \begin{tabular}{|l|l|}
0.728 \\
\end{tabular} & \begin{tabular}{|l|}
0.587 \\
\end{tabular} & 647 & 0.616 & 0.710 & 37 & \begin{tabular}{ll|}
623 & \\
\end{tabular} & 0.589 & 0.521 \\
\hline CD44 & \begin{tabular}{|l|}
0.285 \\
\end{tabular} & 409 & 273 & 181 & 242 & 207 & 600 & 0.649 & 838 & 778 & 674 & 1 & 0.770 & 746 & 0.727 & 660 & 713 & \begin{tabular}{|l|}
0.696 \\
\end{tabular} & \begin{tabular}{|l|}
0.591 \\
\end{tabular} & \begin{tabular}{|l|l|}
0.634 \\
\end{tabular} & .618 & \begin{tabular}{|l|}
0.642 \\
\end{tabular} & 52 & .525 & 0.449 & 0.649 \\
\hline MMP19 & 566 & 557 & 39 & 431 & 446 & 05 & 0.803 & 367 & 60 & 44 & 332 & 0.770 & & 0.902 & 97 & 34 & 744 & \begin{tabular}{|l|l|}
0.754 \\
\end{tabular} & 0.761 & 07 & 37 & 799 & 4 & 660 & .636 & .673 \\
\hline EMILIN2 & 753 & 726 & 767 & 636 & 536 & 671 & \begin{tabular}{|l|l|}
0.662 \\
\end{tabular} & 0.720 & \begin{tabular}{l|l|}
736 \\
\end{tabular} & 768 & 715 & 746 & 902 & & \begin{tabular}{|l|}
0.931 \\
\end{tabular} & .906 & 820 & \begin{tabular}{|l|l|}
0.789 \\
\end{tabular} & 0.836 & 758 & 0.755 & 0.820 & 4 & 689 & 0.713 & ) .716 \\
\hline CTSB & \begin{tabular}{|l|}
0.709 \\
\end{tabular} & 649 & 0.679 & \begin{tabular}{|l|l|}
0.558 \\
\end{tabular} & \begin{tabular}{|l|l|}
0.463 \\
\end{tabular} & 579 & 0.622 & 0.693 & $\begin{array}{l}706 \\
\end{array}$ & 763 & \begin{tabular}{|l|}
0.790 \\
\end{tabular} & 0.727 & 897 & 931 & 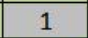 & \begin{tabular}{|l|}
0.942 \\
\end{tabular} & \begin{tabular}{|l|l|}
0.770 \\
\end{tabular} & \begin{tabular}{|l|}
0.847 \\
\end{tabular} & 0.842 & \begin{tabular}{|l|l|}
0.717 \\
\end{tabular} & 0.831 & \begin{tabular}{|l|l|}
0.814 \\
\end{tabular} & 303 & .773 & 0.717 & 0.641 \\
\hline CTSD & \begin{tabular}{|l|}
0.691 \\
\end{tabular} & 671 & 0.616 & \begin{tabular}{|l|l|}
0.565 \\
\end{tabular} & \begin{tabular}{|l|l|}
0.480 \\
\end{tabular} & \begin{tabular}{|l|l|}
0.587 \\
\end{tabular} & \begin{tabular}{|l|l|}
0.677 \\
\end{tabular} & 47 & 996 & 0. & \begin{tabular}{|l|l|}
0.754 \\
\end{tabular} & \begin{tabular}{|l|}
0.660 \\
\end{tabular} & 934 & 66 & \begin{tabular}{|l|}
0.942 \\
\end{tabular} & 1 & 0.704 & \begin{tabular}{|l|l|}
0.791 \\
\end{tabular} & 0.806 & c & 08 & \begin{tabular}{|l|}
0.812 \\
\end{tabular} & 50 & 61 & 33 & .651 \\
\hline 194 & & 3 & & 55 & 403 & & & & & & 0. & 3 & & & 70 & 04 & 1 & \begin{tabular}{|l|l|}
0.753 \\
\end{tabular} & 323 & & 0.607 & \begin{tabular}{|l|}
0.872 \\
\end{tabular} & & 740 & \begin{tabular}{|l|l|}
0.704 \\
\end{tabular} & \\
\hline PPIB & 38 & 34 & 87 & 562 & 0.534 & 35 & 556 & 595 & 803 & 740 & 0.728 & 0.696 & 754 & 789 & \begin{tabular}{|l|l|}
0.847 \\
\end{tabular} & \begin{tabular}{|l|}
0.791 \\
\end{tabular} & \begin{tabular}{|l|l|}
0.753 \\
\end{tabular} & & 0.759 & 0.784 & 0.727 & 0.832 & 741 & 0.878 & 0.567 & 7750 \\
\hline ITGA3 & 51 & 551 & 110 & 634 & 543 & 305 & 530 & 569 & 503 & 442 & 0.587 & 0.591 & 0.761 & 336 & 0.842 & 0.806 & 0.823 & 0.759 & & 0.790 & 0.731 & 0.783 & 871 & .739 & 0.669 & 0.702 \\
\hline CAPN1 & 575 & 0.736 & 0.803 & 669 & 0.745 & 700 & 0.632 & 541 & 515 & 587 & 647 & 0.634 & 707 & 758 & \begin{tabular}{|l|l|} 
\\
\end{tabular} & 667 & \begin{tabular}{|l|l|}
0.749 \\
\end{tabular} & \begin{tabular}{|l|l|}
0.784 \\
\end{tabular} & \begin{tabular}{|l|l|}
0.790 \\
\end{tabular} & & 0.645 & 0.808 & 49 & .728 & 0.489 & 0.834 \\
\hline NCSTN & \begin{tabular}{|l|l|}
0.587 \\
\end{tabular} & 493 & 0.553 & 416 & 398 & \begin{tabular}{|l|l|}
0.468 \\
\end{tabular} & 0.480 & 540 & 536 & 575 & 616 & 0.618 & 737 & 755 & \begin{tabular}{|l|}
0.831 \\
\end{tabular} & 808 & 607 & \begin{tabular}{|l|l|}
0.727 \\
\end{tabular} & \begin{tabular}{|l|l|}
0.731 \\
\end{tabular} & \begin{tabular}{|l|}
0.645 \\
\end{tabular} & 1 & 0.706 & 748 & 0.768 & 0.785 & 0.664 \\
\hline CALR & 575 & \begin{tabular}{|l|}
0.601 \\
\end{tabular} & 0.641 & \begin{tabular}{|l|}
0.501 \\
\end{tabular} & 0.523 & \begin{tabular}{|l|l|}
0.493 \\
\end{tabular} & \begin{tabular}{|l|}
0.582 \\
\end{tabular} & 0.628 & 691 & 665 & 0.710 & 0.642 & 799 & 820 & \begin{tabular}{|l|l|}
0.814 \\
\end{tabular} & 0.812 & 0.872 & \begin{tabular}{|l|}
0.832 \\
\end{tabular} & 0.783 & 0.808 & 0.706 & 1 & 0.745 & \begin{tabular}{|l|}
0.856 \\
\end{tabular} & 0.687 & 0.813 \\
\hline COLGALT1 & 0.512 & 0.523 & 0.549 & 0.455 & 0.346 & \begin{tabular}{|l|l|}
0.592 \\
\end{tabular} & \begin{tabular}{|l|}
0.463 \\
\end{tabular} & 0.528 & 0.664 & 0.693 & 0.587 & 0.752 & 777 & 0.814 & \begin{tabular}{|l|}
0.803 \\
\end{tabular} & 0.750 & 0.850 & 0.741 & \begin{tabular}{|l|l|}
0.871 \\
\end{tabular} & \begin{tabular}{|l|l|}
0.749 \\
\end{tabular} & \begin{tabular}{|l|l|}
0.748 \\
\end{tabular} & 0.745 & 1 & \begin{tabular}{|l|l|}
0.737 \\
\end{tabular} & 0.765 & -0.770 \\
\hline PLOD1 & 0.555 & \begin{tabular}{|l|l|}
0.538 \\
\end{tabular} & 0.592 & \begin{tabular}{|l|l|}
0.484 \\
\end{tabular} & 0.463 & \begin{tabular}{|l|l|}
0.454 \\
\end{tabular} & \begin{tabular}{|l|}
0.464 \\
\end{tabular} & 0.504 & 0.611 & 0.522 & \begin{tabular}{|l|}
0.623 \\
\end{tabular} & 0.525 & 0.660 & 0.689 & \begin{tabular}{|l|}
0.773 \\
\end{tabular} & \begin{tabular}{|l|}
0.761 \\
\end{tabular} & 0.740 & \begin{tabular}{|l|}
0.878 \\
\end{tabular} & 0.739 & \begin{tabular}{|l|}
0.728 \\
\end{tabular} & \begin{tabular}{|l|}
0.768 \\
\end{tabular} & 0.856 & .737 & 1 & \begin{tabular}{|l|l|}
0.718 \\
\end{tabular} & $\mid-0.726$ \\
\hline P4HB & 0.466 & \begin{tabular}{|l|l|} 
& 0.278 \\
\end{tabular} & 0.498 & 0.327 & 0.069 & \begin{tabular}{|l|l|}
0.324 \\
\end{tabular} & 0.536 & 0.685 & 0.334 & 0.452 & 0.589 & 0.449 & 0.636 & 0.713 & 0.717 & 0.633 & 0.704 & 0.567 & 0.669 & 0.489 & 0.785 & 0.687 & 0.765 & 0.718 & 1 & 0.547 \\
\hline FGF2 & $\mid-0.510$ & -0.550 & -0.599 & \begin{tabular}{|l|}
-0.473 \\
\end{tabular} & -0.540 & \begin{tabular}{|l|}
-0.573 \\
\end{tabular} & $\mid-0.490$ & -0.514 & -0.692 & $\mid-0.579$ & -0.521 & -0.649 & -0.673 & -0.716 & $\mid-0.641$ & \begin{tabular}{|c|}
-0.651 \\
\end{tabular} & -0.804 & -0.750 & -0.702 & $|-0.834|$ & \begin{tabular}{|l|}
-0.664 \\
\end{tabular} & $\mid-0.813$ & -0.770 & $|-0.726|$ & -0.547 & \\
\hline
\end{tabular}

${ }^{1}$ Values are Pearson R's correlation coefficients: green fill $r \geq 0.700$; red fill $r \leq-0.700$. VLCD $\mathrm{n}=26$. CALR, calreticulin; CAPN1, calpain 1; CD44, CD44 molecule; CHI3L1, chitinase 3-like 1; COLGALT1, collagen beta(1-O)galactosyltransferase 1; CTSB cathepsin B; CTSD, cathepsin D; CTSS, cathepsin S; ECM, extracellular matrix; EMILIN2, elastin microfibril interface 2; FGF2, fibroblast growth factor 2; ITGA3, integrin, alpha 3; ITGAL, integrin, alpha L; ITGAM, integrin, alpha M; ITGAX, integrin, al pha X; ITGB2, integrin, beta 2; MMP19, matrix metallopeptidase 19; MMP9, matrix metallopeptidase 9; NCSTN, nicastrin; P4HB, prolyl 4-hydroxylase, beta polypeptide; PDIA4, protein disulfide isomerase family A, member 4; PLOD1, procollagen-lysine, 2oxoglutarate 5-dioxygenase 1; PPIB, peptidylprolyl isomerase B; SPP1, secreted phosphoprotein 1; TGFB1, transforming growth factor, beta 1, TGFBI, transforming growth factor, betainduced; TIMP1, TIMP metallopeptidase inhibitor 1; VLCD, very-low-calorie diet. 


\section{Discussion}

The present study focussed on determining the relation between weight regain and changes in expression of ECM-related genes. Significant correlations ( $P \leq 0.01)$ with the WR\% were observed for 25 of the 277 genes. The highest number of correlations was observed in the VLCD WS phase. Five of the positively correlated genes of this phase appeared to belong to a group of 26 genes of which the expression changes correlated highly $(r \geq 0.7, \mathrm{P} \leq 0.001)$ among each other. This group could be divided into three subclusters, one of which is mainly composed of leukocyte-specific integrin genes.

The smallest subcluster contains 4 integrins, which are specific for leukocytes and form the Mac-1 and LFA-1 surface proteins (24). Besides leukocyte-specific integrin genes, the subcluster contains three additional genes: transforming growth factor beta 1 (TGFB1), chitinase 3 like 1 (CHI3L1) and cathepsin S (CTSS). TGFB1 influences the inflammatory status by regulating cytokine levels as was shown for adipose-derived mesenchymal stromal cells in vitro (25). Furthermore, TGFB1 may play a role in leukocyte migration since it has been shown to influence adhesion of leukocytes to orbital fibroblasts (26). The product of the CHI3L1 gene, also known as YKL-40, is a BMI-independent marker for type 2 diabetes (27) produced by macrophages and neutrophils $(28,29)$. YKL-40 inhibits degradation of type I collagen and was suggested to play a role in macrophage infiltration. CTSS is one of eleven cathepsin proteases, which is active in ECM remodelling (30). Adipose tissue CTSS mRNA levels are associated with BMI, suggesting that the CTSS gene is mainly active in adipocytes (31). Also, activated macrophages in interaction with adipocytes increase CTSS production pointing to a close relation between CTSS gene activity and inflammatory status of the adipose tissue (32). Altogether, the gene expression changes of the small subcluster comply with a lowering of leukocytespecific integrins. At the moment we cannot determine if down-regulation is caused by a lower RNAproduction of resident leukocytes or by emigration of leukocytes. Gene expression of monocyte chemoattractant protein $1(M C P 1)$ is down-regulated $(F C=-1.29, Q=0.04)$, whereas expression of macrophage migration inhibitory factor (MIF) does not change during the WS phase. Neutrophils respond to the chemotactic factor $\mathrm{C} 5 \mathrm{a}$ (33). In the present study the $\mathrm{C} 5$ gene in the adipose tissue is significantly down-regulated ( $F C=-1.15, Q=0.01)$ as is the gene for its receptor C5AR1 (FC=-1.44, $Q=0.008$ ). It indicates a lowering of diapedesis of neutrophils during WS. Since in the obese the adipose tissue is in a state of chronic inflammation, the changes in gene activity described here may be related to a reduction of the inflammatory status during the WS period. Analysis of the expression of surface marker genes indicates that lowering of gene expression refers in particular to cells of the myeloid lineage (Supplemental Table 3).

The genes in the second cluster also point to the importance of leukocytes and their involvement in inflammation. In human adipose-derived mesenchymal stromal cells expression of the secreted phosphoprotein 1 (SPP1) gene, of which the protein is also known as osteopontin (OPN), is induced by TGFB1 (25). OPN is a pro-inflammatory cytokine that promotes tissue infiltration of monocytes. Mice lacking OPN show a decreased macrophage infiltration in the adipose tissue and a decreased level of inflammation, but insulin sensitivity is higher compared to normal mice (34). OPN is the ligand for the receptor CD44, which is another member of the cluster. Similar to SPP1-KO mice, CD44-KO mice with high fat diet-induced obesity display lower levels of adipose tissue inflammation and are protected against insulin resistance (35). In humans, CD44 density on adipose tissue macrophages was associated with their pro-inflammatory status. Another member of the second cluster, but also part of the overlapping third cluster is PPIB, also known as cyclophilin B. Gene 
expression of PPIB during the WS phase was positively correlated with WR\% in the VLCD group. Cyclophilins A and B possess chemotactic activity towards various types of leukocytes (36). Cyclophilin B accumulates in the ECM, but can be cleaved off by MMPs. Its signal receptor is CD147, encoded by the gene $B S G$, and is known as the extracellular MMP inducer $(37,38)$. MMP activity is also influenced by OPN, because in the OPN-KO mice a reduced MMP2 and MMP9 activity was observed together with reduced ECM remodelling (39). In the present study, MMP9 is on the intersection between the small and the two larger clusters. A role in adipose tissue remodelling has been suggested for MMP19, because MMP19-KO mice develop diet-induced obesity with hypertrophic adipocytes (40). Besides PPIB, MMP9 and MMP19, the second cluster contains other genes that function as processing enzymes: cathepsin $B(C T S B), C T S D$ and protein disulfide isomerise family A member 4 (PDIA4). Apparently, the second cluster harbours genes for leukocyte activity, insulin sensitivity and ECM remodelling. Both the leukocyte infiltration-related genes SPP1, CD44, CD33 and BSG/CD147 as well as the ECM remodelling genes MMP9, MMP19, CTSB and CTSD are upregulated during $W L$ and downregulated during WS. It suggests that while increased leukocyte infiltration with ECM remodelling occurs during WL, the reverse may happen during WS with reduction of leukocytes and ECM remodelling. Our clustering analysis indicates that both processes are linked suggesting that people with a stronger downregulation of ECM-remodelling may retain more immune cells and have higher risk of weight regain.

Most of the genes coding for processing enzymes of the second cluster are in overlap with the third cluster which harbours three more protein processing genes: collagen beta (1O)galactosyltransferase 1 (COLGALT1), procollagen-lysine,2-oxoglutarate 5-dioxygenase 1 (PLOD1) and prolyl 4-hydroxylase subunit beta $(P 4 H B)$. These genes are involved in modification of proteins including collagens. PLOD1, located in the endoplasmic reticulum, hydroxylates lysine residues in procollagen, whereas COLGALT1 binds galactose residues to hydroxylysine residues of collagen. P4HB can hydroxylate proline residues, which is a prerequisite for collagen fibre formation. Although these observations indicate that the third cluster is involved in ECM modification, there is also a link with insulin sensitivity, for instance through the nicastrin (NCSTN) gene. Adipocyte-specific loss-offunction of NCSTN reduces adipose insulin sensitivity (41). Altogether, the genes in the three connected and overlapping subclusters identify two biological processes in adipose tissue related to weight regain: inflammation and ECM remodelling, with a link to insulin sensitivity.

To the best of our knowledge this is the first report indicating the importance of adipose tissue leukocytes for the risk of weight regain after WL. Goyenechea et al. reported about whole body proinflammatory status in relation to weight regain by showing that subjects regaining weight had higher serum concentrations of TNF- $\alpha$ and mRNA levels of TNF- $\alpha$ and NF-KB subunits in PBMC (42). However, these measurements were done after $W L$, thus under the influence of a negative energy balance. Capel et al. used a different study design with a VLCD followed by a LCD, then followed by three months weight maintenance (15). They distinguished between expression changes of adipocyte metabolic genes and macrophage inflammatory-related genes. Seven of our clustered genes were related by them to macrophage activity during DI: CTSB, CTSS, ITGAM, MMP9, MMP19, SPP1 and TGFBI.

The current findings point to the importance of leukocytes in the adipose tissue for weight regain. A lower reduction of the expression of certain leukocyte integrin genes shortly after WL leads to a 
higher risk, which seems linked to ECM remodelling. Possibly, stronger reduction of ECM remodelling capacity during the WS phase leads to more retention of immune cells. It suggests that resident inflammation after WL increases the risk for weight regain, and might contribute to the worsening of the physiological condition during weight cycling (43). However, the present study can provide only indicative results based on gene expression. Furthermore, adipose tissue biopsies were used containing adipocytes as well as stromal vascular cells, which can obscure the exact contribution of each cell type in the tissue to the biological processes involved. Definite conclusions require further experimental proof, preferably performed with purified adipose tissue cell fractions. 


\section{References}

1. Kahn SE, Hull RL, Utzschneider KM. Mechanisms linking obesity to insulin resistance and type 2 diabetes. Nature, 2006. 444(7121):840-6.

2. Narayan KM, Boyle JP, Thompson TJ, Gregg EW, Williamson DF. Effect of BMI on lifetime risk for diabetes in the U.S. Diabetes Care, 2007. 30(6):1562-6.

3. Horton ES. Effects of lifestyle changes to reduce risks of diabetes and associated cardiovascular risks: results from large scale efficacy trials. Obesity (Silver Spring), 2009. 17 Suppl 3:S43-8.

4. King RJ, Ajjan RA. Vascular risk in obesity: Facts, misconceptions and the unknown. Diab Vasc Dis Res, 2017. 14(1):2-13.

5. Weiss EC, Galuska DA, Kettel Khan L, Gillespie C, Serdula MK. Weight regain in U.S. adults who experienced substantial weight loss, 1999-2002. American journal of preventive medicine, 2007. 33(1):34-40.

6. Sarlio-Lahteenkorva S, Rissanen A, Kaprio J. A descriptive study of weight loss maintenance: 6 and 15 year follow-up of initially overweight adults. Int J Obes Relat Metab Disord, 2000. 24(1):116-25.

7. Barte JC, ter Bogt NC, Bogers RP, Teixeira PJ, Blissmer B, Mori TA, Bemelmans WJ. Maintenance of weight loss after lifestyle interventions for overweight and obesity, a systematic review. Obes Rev, 2010. 11(12):899-906.

8. $\quad \mathrm{Wu} T$, Gao X, Chen M, van Dam RM. Long-term effectiveness of diet-plus-exercise interventions vs. diet-only interventions for weight loss: a meta-analysis. Obes Rev, 2009. 10(3):313-23.

9. MacLean PS, Higgins JA, Giles ED, Sherk VD, Jackman MR. The role for adipose tissue in weight regain after weight loss. Obes Rev, 2015. 16 Suppl 1:45-54.

10. Mariman EC. An adipobiological model for weight regain after weight loss. Adipobiology, 2011. 3(1):915.

11. Wang P, Bouwman FG, Mariman EC. Generally detected proteins in comparative proteomics--a matter of cellular stress response? Proteomics, 2009. 9(11):2955-66.

12. Roumans NJ, Camps SG, Renes J, Bouwman FG, Westerterp KR, Mariman EC. Weight loss-induced stress in subcutaneous adipose tissue is related to weight regain. Br J Nutr, 2016. 115(5):913-20.

13. Roumans NJ, Vink RG, Gielen M, Zeegers MP, Holst C, Wang P et al. Variation in extracellular matrix genes is associated with weight regain after weight loss in a sex-specific manner. Genes Nutr, 2015. 10(6):56.

14. Choe SS, Huh JY, Hwang IJ, Kim JI, Kim JB. Adipose Tissue Remodeling: Its Role in Energy Metabolism and Metabolic Disorders. Frontiers in endocrinology, 2016. 7:30.

15. Capel F, Klimcakova E, Viguerie N, Roussel B, Vitkova M, Kovacikova M et al. Macrophages and adipocytes in human obesity: adipose tissue gene expression and insulin sensitivity during calorie restriction and weight stabilization. Diabetes, 2009. 58(7):1558-67.

16. Johansson LE, Danielsson AP, Parikh H, Klintenberg M, Norstrom F, Groop L, Ridderstrale $M$. Differential gene expression in adipose tissue from obese human subjects during weight loss and weight maintenance. The American journal of clinical nutrition, 2012. 96(1):196-207.

17. Sramkova V, Rossmeislova L, Krauzova E, Kracmerova J, Koc M, Langin D, Stich V, Siklova M. Comparison of early ( 2 days) and later (28 days) response of adipose tissue to very-low calorie diet in obese women. The Journal of clinical endocrinology and metabolism, 2016.jc20162161.

18. Vink RG, Roumans NJ, Arkenbosch LA, Mariman EC, van Baak MA. The effect of rate of weight loss on long-term weight regain in adults with overweight and obesity. Obesity (Silver Spring), 2016. 24(2):321-7.

19. Richtlijnen voedselkeuze. http://www.voedingscentrum.nl/professionals/schijf-vanvijf/Richtlijnen.aspx, 2011.

20. Dempster $\mathrm{P}$, Aitkens S. A new air displacement method for the determination of human body composition. Med Sci Sports Exerc, 1995. 27(12):1692-7.

21. Siri WE. The gross composition of the body. Advances in biological and medical physics, 1956. 4:23980.

22. Smyth GK. Limma: linear models for microarray data. Edtion ed. Bioinformatics and computational biology solutions using R and Bioconductor: Springer, 2005:397-420.

23. Xiao Q, Jiang Y, Liu Q, Yue J, Liu C, Zhao X, Qiao Y, Ji H, Chen J, Ge G. Minor Type IV Collagen alpha5 Chain Promotes Cancer Progression through Discoidin Domain Receptor-1. PLoS genetics, 2015. 11(5):e1005249.

24. Hynes RO. Integrins: bidirectional, allosteric signaling machines. Cell, 2002. 110(6):673-87. 
25. Rodriguez TM, Saldias A, Irigo M, Zamora JV, Perone MJ, Dewey RA. Effect of TGF-beta1 Stimulation on the Secretome of Human Adipose-Derived Mesenchymal Stromal Cells. Stem cells translational medicine, 2015. 4(8):894-8.

26. Guo N, Woeller CF, Feldon SE, Phipps RP. Peroxisome proliferator-activated receptor gamma ligands inhibit transforming growth factor-beta-induced, hyaluronan-dependent, T cell adhesion to orbital fibroblasts. The Journal of biological chemistry, 2011. 286(21):18856-67.

27. Nielsen AR, Erikstrup C, Johansen JS, Fischer CP, Plomgaard P, Krogh-Madsen R, Taudorf S, Lindegaard B, Pedersen BK. Plasma YKL-40: a BMI-independent marker of type 2 diabetes. Diabetes, 2008. 57(11):3078-82.

28. Volck B, Price PA, Johansen JS, Sorensen O, Benfield TL, Nielsen HJ, Calafat J, Borregaard N. YKL-40, a mammalian member of the chitinase family, is a matrix protein of specific granules in human neutrophils. Proceedings of the Association of American Physicians, 1998. 110(4):351-60.

29. Johansen JS. Studies on serum YKL-40 as a biomarker in diseases with inflammation, tissue remodelling, fibroses and cancer. Danish medical bulletin, 2006. 53(2):172-209.

30. Sage J, Leblanc-Noblesse E, Nizard C, Sasaki T, Schnebert S, Perrier E, Kurfurst R, Bromme D, Lalmanach $G$, Lecaille $F$. Cleavage of nidogen-1 by cathepsin $S$ impairs its binding to basement membrane partners. PloS one, 2012. 7(8):e43494.

31. Taleb S, Lacasa D, Bastard JP, Poitou C, Cancello R, Pelloux V et al. Cathepsin S, a novel biomarker of adiposity: relevance to atherogenesis. FASEB journal : official publication of the Federation of American Societies for Experimental Biology, 2005. 19(11):1540-2.

32. Taleb S, Clement K. Emerging role of cathepsin S in obesity and its associated diseases. Clinical chemistry and laboratory medicine, 2007. 45(3):328-32.

33. Tralau T, Meyer-Hoffert U, Schroder JM, Wiedow O. Human leukocyte elastase and cathepsin G are specific inhibitors of C5a-dependent neutrophil enzyme release and chemotaxis. Experimental dermatology, 2004. 13(5):316-25.

34. Nomiyama T, Perez-Tilve D, Ogawa D, Gizard F, Zhao Y, Heywood EB et al. Osteopontin mediates obesity-induced adipose tissue macrophage infiltration and insulin resistance in mice. The Journal of clinical investigation, 2007. 117(10):2877-88.

35. Liu LF, Kodama K, Wei K, Tolentino LL, Choi O, Engleman EG, Butte AJ, McLaughlin T. The receptor CD44 is associated with systemic insulin resistance and proinflammatory macrophages in human adipose tissue. Diabetologia, 2015. 58(7):1579-86.

36. Sherry B, Yarlett N, Strupp A, Cerami A. Identification of cyclophilin as a proinflammatory secretory product of lipopolysaccharide-activated macrophages. Proceedings of the National Academy of Sciences of the United States of America, 1992. 89(8):3511-5.

37. Yurchenko V, Constant S, Eisenmesser E, Bukrinsky M. Cyclophilin-CD147 interactions: a new target for anti-inflammatory therapeutics. Clinical and experimental immunology, 2010. 160(3):305-17.

38. Marcant A, Denys A, Melchior A, Martinez P, Deligny A, Carpentier M, Allain F. Cyclophilin B attenuates the expression of TNF-alpha in lipopolysaccharide-stimulated macrophages through the induction of $B$ cell lymphoma-3. Journal of immunology, 2012. 189(4):2023-32.

39. Lancha A, Rodriguez A, Catalan V, Becerril S, Sainz N, Ramirez B, Burrell MA, Salvador J, Fruhbeck G, Gomez-Ambrosi J. Osteopontin deletion prevents the development of obesity and hepatic steatosis via impaired adipose tissue matrix remodeling and reduced inflammation and fibrosis in adipose tissue and liver in mice. PloS one, 2014. 9(5):e98398.

40. Pendas AM, Folgueras AR, Llano E, Caterina J, Frerard F, Rodriguez F, Astudillo A, Noel A, BirkedalHansen $H$, Lopez-Otin C. Diet-induced obesity and reduced skin cancer susceptibility in matrix metalloproteinase 19-deficient mice. Molecular and cellular biology, 2004. 24(12):5304-13.

41. Sparling DP, Yu J, Kim K, Zhu C, Brachs S, Birkenfeld AL, Pajvani UB. Adipocyte-specific blockade of gamma-secretase, but not inhibition of Notch activity, reduces adipose insulin sensitivity. Molecular metabolism, 2016. 5(2):113-21.

42. Goyenechea E, Parra D, Crujeiras AB, Abete I, Martinez JA. A nutrigenomic inflammation-related PBMC-based approach to predict the weight-loss regain in obese subjects. Annals of nutrition \& metabolism, 2009. 54(1):43-51.

43. Montani JP, Viecelli AK, Prevot A, Dulloo AG. Weight cycling during growth and beyond as a risk factor for later cardiovascular diseases: the 'repeated overshoot' theory. International journal of obesity, 2006. 30 Suppl 4:S58-66. 
Supplementary Figures and Tables 
Supplement Table 1: List of the 277 candidate extracellular matrix related genes used for the analysis.

\begin{tabular}{|c|c|c|c|}
\hline Gene ID & Gene name & Gene \# & Chromosome \\
\hline $\mathrm{A} 2 \mathrm{M}$ & alpha-2-macroglobulin & 2 & 12 \\
\hline ABI3BP & ABI family, member 3 (NESH) binding protein & 25890 & 3 \\
\hline ACTN1 & actinin, alpha 1 & 87 & 14 \\
\hline ADAM10 & ADAM metallopeptidase domain 10 & 102 & 15 \\
\hline ADAM11 & ADAM metallopeptidase domain 11 & 4185 & 17 \\
\hline ADAM12 & ADAM metallopeptidase domain 12 & 8038 & 10 \\
\hline ADAM15 & ADAM metallopeptidase domain 15 & 8751 & 1 \\
\hline ADAM17 & ADAM metallopeptidase domain 17 & 6868 & 2 \\
\hline ADAM19 & ADAM metallopeptidase domain 19 & 8728 & 5 \\
\hline ADAM22 & ADAM metallopeptidase domain 22 & 53616 & 7 \\
\hline ADAM8 & ADAM metallopeptidase domain 8 & 101 & 10 \\
\hline ADAM9 & ADAM metallopeptidase domain 9 & 8754 & 8 \\
\hline ADAMTS1 & ADAM metallopeptidase with thrombospondin type 1 motif, 1 & 9510 & 21 \\
\hline ADAMTS14 & ADAM metallopeptidase with thrombospondin type 1 motif, 14 & 140766 & 10 \\
\hline ADAMTS2 & ADAM metallopeptidase with thrombospondin type 1 motif, 2 & 9509 & 5 \\
\hline ADAMTS4 & ADAM metallopeptidase with thrombospondin type 1 motif, 4 & 9507 & 1 \\
\hline ADAMTS5 & ADAM metallopeptidase with thrombospondin type 1 motif, 5 & 11096 & 21 \\
\hline ADAMTSL4 & ADAMTS-like 4 & 54507 & 1 \\
\hline AGRN & Agrin & 375790 & 1 \\
\hline AGT & angiotensinogen (serpin peptidase inhibitor, clade A, member 8) & 183 & 1 \\
\hline APBB2 & amyloid beta (A4) precursor protein-binding, family B, member 2 & 323 & 4 \\
\hline AZGP1 & alpha-2-glycoprotein 1, zinc-binding & 563 & 7 \\
\hline BCL3 & B-cell CLL/lymphoma 3 & 602 & 19 \\
\hline BGN & Biglycan & 633 & $\mathrm{x}$ \\
\hline BMP1 & bone morphogenetic protein 1 & 649 & 8 \\
\hline BMP2 & bone morphogenetic protein 2 & 650 & 20 \\
\hline BMP4 & bone morphogenetic protein 4 & 652 & 14 \\
\hline BSG & basigin (Ok blood group) & 682 & 19 \\
\hline CALR & Calreticulin & 811 & 19 \\
\hline CAPN1 & calpain $1,(\mathrm{mu} / \mathrm{l})$ large subunit & 823 & 11 \\
\hline CASP3 & caspase 3 , apoptosis-related cysteine peptidase & 836 & 4 \\
\hline CCDC80 & coiled-coil domain containing 80 & 151887 & 3 \\
\hline CD151 & CD151 molecule (Raph blood group) & 977 & 11 \\
\hline CD44 & CD44 molecule (Indian blood group) & 960 & 11 \\
\hline CD47 & CD47 molecule & 961 & 3 \\
\hline CER1 & cerberus 1, DAN family BMP antagonist & 9350 & 9 \\
\hline CHI3L1 & chitinase 3-like 1 (cartilage glycoprotein-39) & 1116 & 1 \\
\hline CIB1 & calcium and integrin binding 1 (calmyrin) & 10519 & 15 \\
\hline CILP & cartilage intermediate layer protein, nucleotide pyrophosphohydrolase & 8483 & 15 \\
\hline CMA1 & chymase 1 , mast cell & 1215 & 14 \\
\hline CNTNAP1 & contactin associated protein 1 & 8506 & 17 \\
\hline COL11A1 & collagen, type XI, alpha 1 & 1301 & 1 \\
\hline COL12A1 & collagen, type XII, alpha 1 & 1303 & 6 \\
\hline COL13A1 & collagen, type XIII, alpha 1 & 1305 & 10 \\
\hline COL14A1 & collagen, type XIV, alpha 1 & 7373 & 8 \\
\hline COL15A1 & collagen, type $X V$, alpha 1 & 1306 & 9 \\
\hline COL16A1 & collagen, type XVI, alpha 1 & 1307 & 1 \\
\hline COL17A1 & collagen, type XVII, alpha 1 & 1308 & 10 \\
\hline COL18A1 & collagen, type XVIII, alpha 1 & 80781 & 21 \\
\hline COL1A1 & collagen, type I, alpha 1 & 1277 & 17 \\
\hline COL1A2 & collagen, type I, alpha 2 & 1278 & 7 \\
\hline COL20A1 & collagen, type $X X$, alpha 1 & 57642 & 20 \\
\hline COL21A1 & collagen, type XXI, alpha 1 & 81578 & 6 \\
\hline COL23A1 & collagen, type XXIII, alpha 1 & 91522 & 5 \\
\hline COL25A1 & collagen, type XXV, alpha 1 & 84570 & 4 \\
\hline COL26A1 & collagen, type XXVI, alpha 1 & 136227 & 7 \\
\hline COL27A1 & collagen, type XXVII, alpha 1 & 85301 & 9 \\
\hline COL2A1 & collagen, type II, alpha 1 & 1280 & 12 \\
\hline COL3A1 & collagen, type III, alpha 1 & 1281 & 2 \\
\hline COL4A1 & collagen, type IV, alpha 1 & 1282 & 13 \\
\hline COL4A2 & collagen, type IV, alpha 2 & 1284 & 13 \\
\hline COL4A5 & collagen, type IV, alpha 5 & 1287 & $x$ \\
\hline COL5A1 & collagen, type $V$, alpha 1 & 1289 & 9 \\
\hline COL5A2 & collagen, type $\mathrm{V}$, alpha 2 & 1290 & 2 \\
\hline COL5A3 & collagen, type $V$, alpha 3 & 50509 & 19 \\
\hline
\end{tabular}




\begin{tabular}{|c|c|c|c|}
\hline COL6A1 & collagen, type $\mathrm{VI}$, alpha 1 & 1291 & 21 \\
\hline COL6A2 & collagen, type $\mathrm{VI}$, alpha 2 & 1292 & 21 \\
\hline COL6A3 & collagen, type VI, alpha 3 & 1293 & 2 \\
\hline COL6A6 & collagen, type $\mathrm{VI}$, alpha 6 & 131873 & 3 \\
\hline COL8A1 & collagen, type VIII, alpha 1 & 1295 & 3 \\
\hline COL8A2 & collagen, type VIII, alpha 2 & 1296 & 1 \\
\hline COL9A2 & collagen, type IX, alpha 2 & 1298 & 1 \\
\hline COL9A3 & collagen, type IX, alpha 3 & 1299 & 20 \\
\hline COLGALT1 & collagen beta(1-0)galactosyltransferase 1 & 79709 & 19 \\
\hline COMP & cartilage oligomeric matrix protein & 1311 & 19 \\
\hline CRISPLD2 & cysteine-rich secretory protein LCCL domain containing 2 & 83716 & 16 \\
\hline CRTAP & cartilage associated protein & 10491 & 3 \\
\hline CSGALNACT1 & chondroitin sulfate $\mathrm{N}$-acetylgalactosaminyltransferase 1 & 55790 & 8 \\
\hline CSPG4 & chondroitin sulfate proteoglycan 4 & 1464 & 15 \\
\hline CST3 & cystatin C & 1471 & 20 \\
\hline CTGF & connective tissue growth factor & 1490 & 6 \\
\hline CTRB1 & chymotrypsinogen B1 & 1504 & 16 \\
\hline CTRB2 & chymotrypsinogen B2 & 440387 & 16 \\
\hline CTSB & cathepsin B & 1508 & 8 \\
\hline CTSD & cathepsin D & 1509 & 11 \\
\hline CTSG & cathepsin G & 1511 & 14 \\
\hline CTSK & cathepsin $\mathrm{K}$ & 1513 & 1 \\
\hline CTSL & cathepsin L & 1514 & 9 \\
\hline CTSS & cathepsin S & 1520 & 1 \\
\hline CYP1B1 & cytochrome P450, family 1 , subfamily B, polypeptide 1 & 1545 & 2 \\
\hline CYR61 & cysteine-rich, angiogenic inducer, 61 & 3491 & 1 \\
\hline DAG1 & dystroglycan 1 (dystrophin-associated glycoprotein 1) & 1605 & 3 \\
\hline $\mathrm{DCN}$ & Decorin & 1634 & 12 \\
\hline DDR1 & discoidin domain receptor tyrosine kinase 1 & 780 & 6 \\
\hline DDR2 & discoidin domain receptor tyrosine kinase 2 & 4921 & 1 \\
\hline DMD & Dystrophin & 1756 & $\mathrm{x}$ \\
\hline DPP4 & dipeptidyl-peptidase 4 & 1803 & 2 \\
\hline DPT & Dermatopontin & 1805 & 1 \\
\hline ECM1 & extracellular matrix protein 1 & 1893 & 1 \\
\hline ECM2 & extracellular matrix protein 2 , female organ and adipocyte specific & 1842 & 9 \\
\hline EFEMP1 & EGF containing fibulin-like extracellular matrix protein 1 & 2202 & 2 \\
\hline EFEMP2 & EGF containing fibulin-like extracellular matrix protein 2 & 30008 & 11 \\
\hline EGFL6 & EGF-like-domain, multiple 6 & 25975 & $x$ \\
\hline EGFLAM & EGF-like, fibronectin type III and laminin $\mathrm{G}$ domains & 133584 & 5 \\
\hline ELANE & elastase, neutrophil expressed & 1991 & 19 \\
\hline ELN & Elastin & 2006 & 7 \\
\hline EMILIN1 & elastin microfibril interfacer 1 & 11117 & 2 \\
\hline EMILIN2 & elastin microfibril interfacer 2 & 84034 & 18 \\
\hline ENG & Endoglin & 2022 & 9 \\
\hline ERCC2 & excision repair cross-complementation group 2 & 2068 & 19 \\
\hline ERO1L & ERO1-like (S. cerevisiae) & 30001 & 14 \\
\hline ERO1LB & ERO1-like beta (S. cerevisiae) & 56605 & 1 \\
\hline ETS1 & v-ets avian erythroblastosis virus E26 oncogene homolog 1 & 2113 & 11 \\
\hline F11R & F11 receptor & 50848 & 1 \\
\hline FAP & fibroblast activation protein, alpha & 2191 & 2 \\
\hline FBLN1 & fibulin 1 & 2192 & 22 \\
\hline FBLN2 & fibulin 2 & 2199 & 3 \\
\hline FBLN5 & fibulin 5 & 10516 & 14 \\
\hline FBN1 & fibrillin 1 & 2200 & 15 \\
\hline FER & fer (fps/fes related) tyrosine kinase & 2241 & 5 \\
\hline FGF2 & fibroblast growth factor 2 (basic) & 2247 & 4 \\
\hline FKBP9 & FK506 binding protein $9,63 \mathrm{kDa}$ & 11328 & 7 \\
\hline FMOD & Fibromodulin & 2331 & 1 \\
\hline FN1 & fibronectin 1 & 2335 & 2 \\
\hline FURIN & furin (paired basic amino acid cleaving enzyme) & 5045 & 15 \\
\hline FZD4 & frizzled class receptor 4 & 8322 & 11 \\
\hline GAS6 & growth arrest-specific 6 & 2621 & 13 \\
\hline GFAP & glial fibrillary acidic protein & 2670 & 17 \\
\hline GFOD2 & glucose-fructose oxidoreductase domain containing 2 & 81577 & 16 \\
\hline GPC1 & glypican 1 & 2817 & 2 \\
\hline GPM6B & glycoprotein M6B & 2824 & $x$ \\
\hline HAS2 & hyaluronan synthase 2 & 3037 & 8 \\
\hline
\end{tabular}




\begin{tabular}{|c|c|c|}
\hline HAS3 & hyaluronan synthase 3 & 3038 \\
\hline HSD17B12 & hydroxysteroid (17-beta) dehydrogenase 12 & 51144 \\
\hline HSPG2 & heparan sulfate proteoglycan 2 & 3339 \\
\hline ICAM1 & intercellular adhesion molecule 1 & 3383 \\
\hline ICAM2 & intercellular adhesion molecule 2 & 3384 \\
\hline ICAM3 & intercellular adhesion molecule 3 & 3385 \\
\hline ITGA1 & integrin, alpha 1 & 3672 \\
\hline ITGA11 & integrin, alpha 11 & 22801 \\
\hline ITGA2 & integrin, alpha 2 (CD49B, alpha 2 subunit of VLA-2 receptor) & 3673 \\
\hline ITGA2B & $\begin{array}{l}\text { integrin, alpha } 2 \mathrm{~b} \text { (platelet glycoprotein Ilb of IIb/IIla complex, antigen } \\
\text { CD41) }\end{array}$ & 3674 \\
\hline ITGA3 & integrin, alpha 3 (antigen CD49C, alpha 3 subunit of VLA-3 receptor) & 3675 \\
\hline ITGA4 & integrin, alpha 4 (antigen CD49D, alpha 4 subunit of VLA-4 receptor) & 3676 \\
\hline ITGA5 & integrin, alpha 5 (fibronectin receptor, alpha polypeptide) & 3678 \\
\hline ITGA6 & integrin, alpha 6 & 3655 \\
\hline ITGA7 & integrin, alpha 7 & 3679 \\
\hline ITGA8 & integrin, alpha 8 & 8516 \\
\hline ITGA9 & integrin, alpha 9 & 3680 \\
\hline ITGAD & integrin, alpha D & 3681 \\
\hline ITGAE & $\begin{array}{l}\text { integrin, alpha E (antigen CD103, human mucosal lymphocyte antigen } 1 \text {; } \\
\text { alpha polypeptide) }\end{array}$ & 3682 \\
\hline ITGAL & $\begin{array}{l}\text { integrin, alpha } L \text { (antigen CD11A ( } 180 \text { ), lymphocyte function-associated } \\
\text { antigen } 1 \text {; alpha polypeptide) }\end{array}$ & 3683 \\
\hline ITGAM & integrin, alpha $\mathrm{M}$ (complement component 3 receptor 3 subunit) & 3684 \\
\hline ITGAV & integrin, alpha $\mathrm{V}$ & 3685 \\
\hline ITGAX & integrin, alpha $X$ (complement component 3 receptor 4 subunit) & 3687 \\
\hline ITGB2 & integrin, beta 2 (complement component 3 receptor 3 and 4 subunit) & 3689 \\
\hline ITGB3 & integrin, beta 3 (platelet glycoprotein IIla, antigen CD61) & 3690 \\
\hline ITGB4 & integrin, beta 4 & 3691 \\
\hline ITGB5 & integrin, beta 5 & 3693 \\
\hline ITGB7 & integrin, beta 7 & 3695 \\
\hline JAM2 & junctional adhesion molecule 2 & 58494 \\
\hline JAM3 & junctional adhesion molecule 3 & 83700 \\
\hline KAZALD1 & Kazal-type serine peptidase inhibitor domain 1 & 81621 \\
\hline KDR & kinase insert domain receptor & 3791 \\
\hline KIF9 & kinesin family member 9 & 64147 \\
\hline LAMA2 & laminin, alpha 2 & 3908 \\
\hline LAMA3 & laminin, alpha 3 & 3909 \\
\hline LAMA5 & laminin, alpha 5 & 3911 \\
\hline LAMB1 & laminin, beta 1 & 3912 \\
\hline LAMB2 & laminin, beta 2 (laminin S) & 3913 \\
\hline LAMB3 & laminin, beta 3 & 3914 \\
\hline LAMC1 & laminin, gamma 1 (formerly LAMB2) & 3915 \\
\hline LAMC3 & laminin, gamma 3 & 10319 \\
\hline LGALS1 & lectin, galactoside-binding, soluble, 1 & 3956 \\
\hline LGALS3BP & lectin, galactoside-binding, soluble, 3 binding protein & 3959 \\
\hline LOX & lysyl oxidase & 4015 \\
\hline LOXL1 & lysyl oxidase-like 1 & 4016 \\
\hline LRP4 & low density lipoprotein receptor-related protein 4 & 4038 \\
\hline LRP5 & low density lipoprotein receptor-related protein 5 & 4041 \\
\hline LTBP1 & latent transforming growth factor beta binding protein 1 & 4052 \\
\hline LTBP3 & latent transforming growth factor beta binding protein 3 & 4054 \\
\hline LTBP4 & latent transforming growth factor beta binding protein 4 & 8425 \\
\hline LUM & Lumican & 4060 \\
\hline MADCAM1 & mucosal vascular addressin cell adhesion molecule 1 & 8174 \\
\hline MATN2 & matrilin 2 & 4147 \\
\hline MATN4 & matrilin 4 & 8785 \\
\hline MFAP2 & microfibrillar-associated protein 2 & 4237 \\
\hline MFAP3 & microfibrillar-associated protein 3 & 4238 \\
\hline MFAP4 & microfibrillar-associated protein 4 & 4239 \\
\hline MFAP5 & microfibrillar associated protein 5 & 8076 \\
\hline MFI2 & antigen p97 (melanoma associated) & 4241 \\
\hline MMP14 & matrix metallopeptidase 14 (membrane-inserted) & 4323 \\
\hline MMP15 & matrix metallopeptidase 15 (membrane-inserted) & 4324 \\
\hline MMP19 & matrix metallopeptidase 19 & 4327 \\
\hline MMP2 & matrix metallopeptidase 2 & 4313 \\
\hline MMP7 & matrix metallopeptidase 7 & 4316 \\
\hline MMP9 & matrix metallopeptidase 9 & 4318 \\
\hline
\end{tabular}




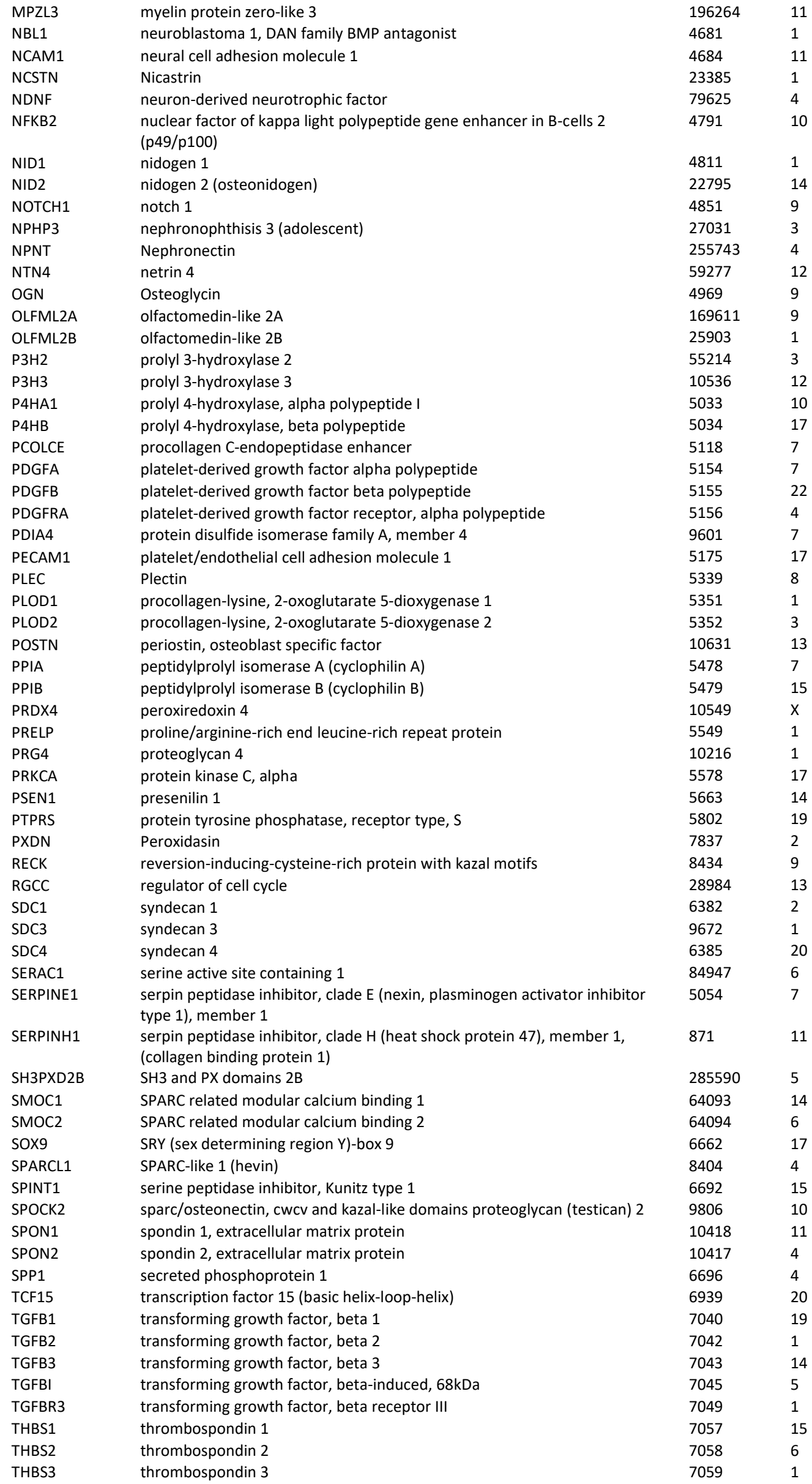


Chapter 3

\begin{tabular}{llll} 
TIMP1 & TIMP metallopeptidase inhibitor 1 & 7076 & X \\
TIMP2 & TIMP metallopeptidase inhibitor 2 & 7077 & 17 \\
TIMP4 & TIMP metallopeptidase inhibitor 4 & 7079 & 3 \\
TLL1 & tolloid-like 1 & 7092 & 4 \\
TNC & tenascin C & 3371 & 9 \\
TNF & tumor necrosis factor & 7124 & 6 \\
TNFRSF11B & tumor necrosis factor receptor superfamily, member 11b & 4982 & 8 \\
TNN & tenascin N & 63923 & 1 \\
TNXB & tenascin XB & 7148 & 6 \\
TRAPPC4 & trafficking protein particle complex 4 & 51399 \\
VCAM1 & vascular cell adhesion molecule 1 & 7412 & 11 \\
VCAN & Versican & 1462 & 1 \\
VIT & Vitrin & 5212 & 2 \\
VWA1 & von Willebrand factor A domain containing 1 & 64856 \\
VWF & von Willebrand factor & 7450 & 1 \\
\hline
\end{tabular}


Supplement Table 2: Correlation matrix of changes in ECM gene expressions with each other during the weight loss phase in the VLCD ${ }^{1}$.

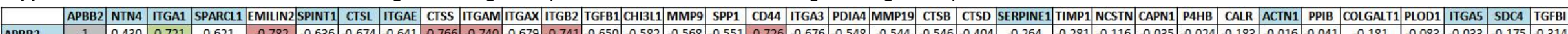

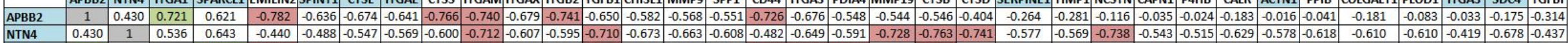

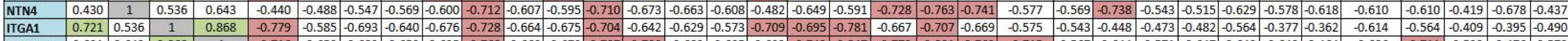

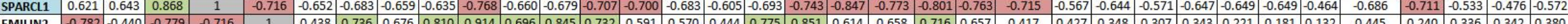

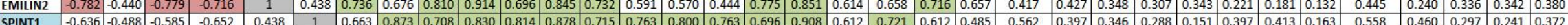

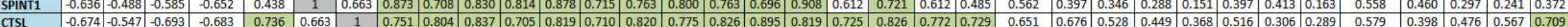

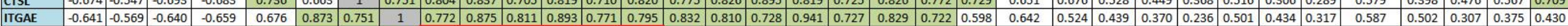

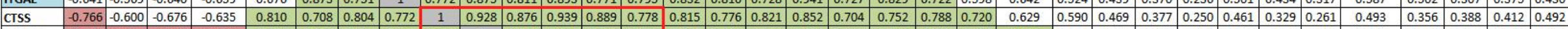

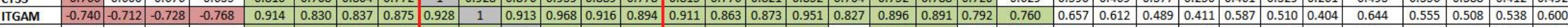

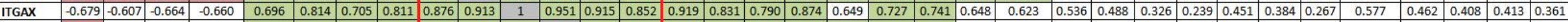

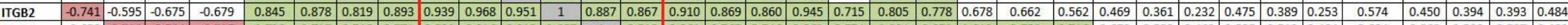

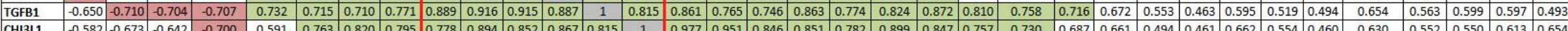

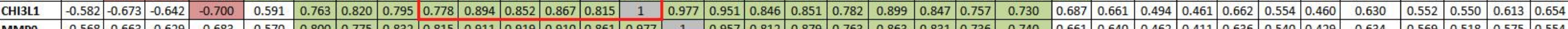

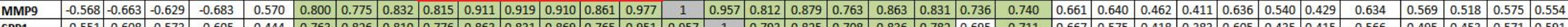

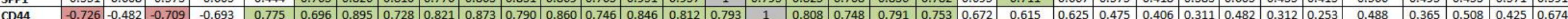

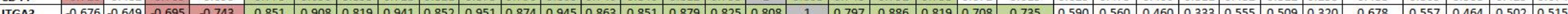

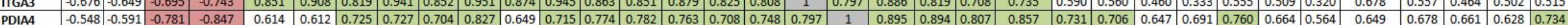

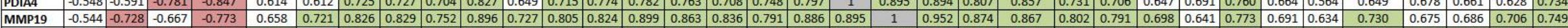

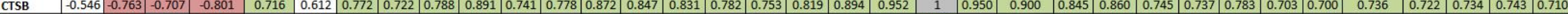

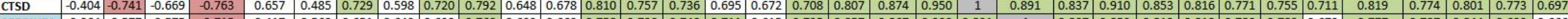

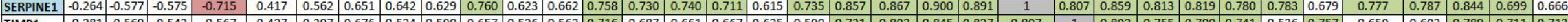

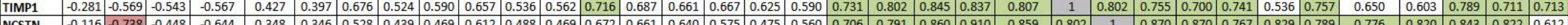

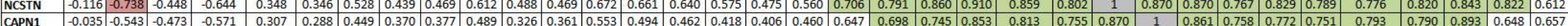

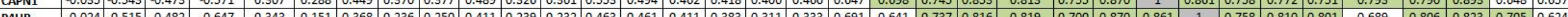

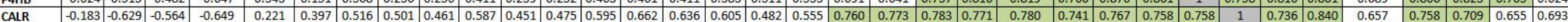

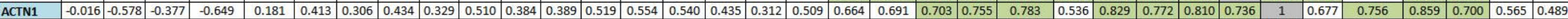

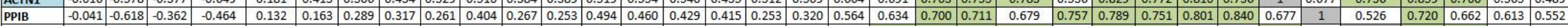

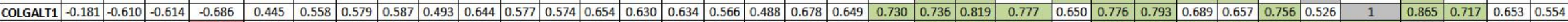

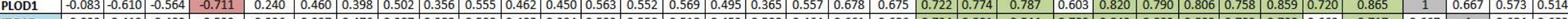

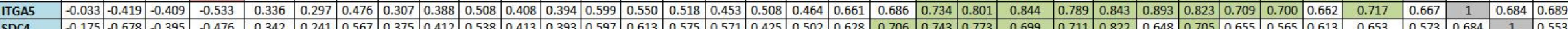

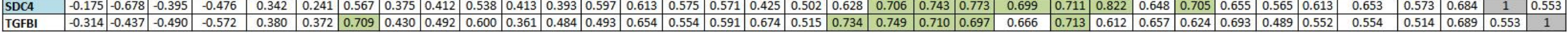
${ }^{1}$ Values are Pearson R's correlation coefficients: green fill $r \geq 0.700$; red fill $r \leq-0.700$. VLCD ( $\left.n=26\right)$. ACTN1, actinin alpha 1; APBB2, amyloid beta precursor protein-binding, family B, member 2; CALR, calreticulin; CAPN1, calpain 1; CD44, CD44 molecule; CHI3L1, chitinase 3 like 1; COLGALT1, collagen beta (1-O)galactosyltransferase 1; CTSB, cathepsin B; CTSD, cathepsin D; CTSL, cathepsin L; CTSS, cathepsin S; EMILIN2, elastin microfibril interface 2; ITGA1, integrin, alpha 1; ITGA3, integrin, alpha 3; ITGA5, integrin, alpha 5; ITGAE, integrin alpha E; ITGAM, integrin alpha M; ITGAX, integrin alpha X; ITGB2, integrin beta 2; MMP19, matrix metallopeptidase 19; MMP9, matrix metallopeptidase 9; NCSTN, nicastrin; OPN, osteopontin; P4HB, prolyl 4-hydroxylase subunit beta; PDIA4, protein disulfide isomerase family A member 4; PLOD1, procollagen-lysine,2-oxoglutarate 5-dioxygenase 1; PPIB, peptidylprolyl isomerase B; SDC4, syndecan 4; SERPINE1, serpin peptidase inhibitor, clade E, member 1; SPARCL1, secreted protein acidic and cysteine rich-like 1; SPINT1, serine peptidase inhibitor kunitz type 1; SPP1, secreted phosphoprotein 1; TGFB1, transforming growth factor beta 1; TGFBI, transforming growth factor, beta-induced; TIMP1, TIMP metallopeptidase inhibitor 1; VLCD, very-low-calorie diet. 
I Supplement Table 3: Expression changes of surface marker genes in the VLCD during the weight stabilisation phase ${ }^{1,2}$.

\begin{tabular}{|c|c|c|c|c|c|c|c|c|c|c|c|c|c|c|c|c|}
\hline \multirow{2}{*}{$\begin{array}{l}\text { Surface } \\
\text { antigen }\end{array}$} & \multirow[t]{2}{*}{ Gene ID } & \multicolumn{9}{|l|}{ Myeloid cells } & \multicolumn{4}{|c|}{ Lymphoid cells } & \multirow[t]{2}{*}{ FC } & \multirow[t]{2}{*}{ Q-value } \\
\hline & & Macrophages & Monocytes & Neutrophils & Basophils & Eosinophils & Granulocytes & Platelets & $\begin{array}{l}\text { Mast } \\
\text { cells }\end{array}$ & $\begin{array}{c}\text { Myeloid } \\
\text { cells }\end{array}$ & NK & $\begin{array}{c}\text { Cytotoxic } \\
\text { T-cell }\end{array}$ & T-cell & $\begin{array}{l}\text { (pre-) } \\
\text { B-cells }\end{array}$ & & \\
\hline CD204 & MSR1 & $x$ & & & & & & & & & & & & & -2.09 & 0.000 \\
\hline CD9 & CD9 & & & & $x$ & $x$ & & $x$ & & & & & & $x$ & -1.51 & 0.000 \\
\hline CD68 & CD68 & $x$ & $x$ & $x$ & $x$ & & & & & $x$ & & & & & -1.61 & 0.000 \\
\hline CD107b & LAMP2 & & & & & & & $x$ & & & & & $x$ & & -1.19 & 0.000 \\
\hline CD107a & LAMP1 & & & & & & $x$ & $x$ & & & & & $x$ & & -1.16 & 0.001 \\
\hline CD11b & ITGAM & & $x$ & $x$ & & & $x$ & & & & $x$ & & $x$ & $x$ & -1.39 & 0.002 \\
\hline CD300a & CD300a & & $x$ & & & & $x$ & & $x$ & & $x$ & & $x$ & $x$ & -1.38 & 0.004 \\
\hline CD163 & CD163 & $x$ & $x$ & & & & & & & & & & & & -1.29 & 0.013 \\
\hline CD11c & ITGAX & $x$ & $x$ & & & & $x$ & & & & & & $x$ & $x$ & -1.54 & 0.014 \\
\hline CD352 & SLAMF6 & & & & & & & & & $x$ & $x$ & & $x$ & $x$ & -1.37 & 0.021 \\
\hline CD14 & CD14 & $x$ & $x$ & & & & $\mathrm{x}$ & & & & & & & & -1.17 & 0.062 \\
\hline CD312 & ADGRE2 & $x$ & $x$ & & & & $x$ & & & & & & & & -1.29 & 0.073 \\
\hline CD281 & TLR1 & $x$ & $x$ & & & & & & & & & & & & -1.20 & 0.078 \\
\hline CD56 & NCAM1 & & & & & & & & & & $x$ & & $x$ & & -1.09 & 0.145 \\
\hline CD3d & CD3d & & & & & & & & & & & & $x$ & & 1.12 & 0.157 \\
\hline CD179b & IGLL1 & & & & & & & & & & & & & $x$ & 1.06 & 0.227 \\
\hline CD1c & $\mathrm{CD} 1 \mathrm{c}$ & & & & & & & & & & & & $x$ & $x$ & 1.10 & 0.231 \\
\hline CD8a & CD8a & & & & & & & & & & $x$ & $x$ & $x$ & & 1.09 & 0.235 \\
\hline CD11a & ITGAL & $x$ & $x$ & & & & & & & & & & & & -1.05 & 0.420 \\
\hline CD3e & CD3e & & & & & & & & & & & & $x$ & & 1.05 & 0.387 \\
\hline CD169 & SIGLEC1 & $x$ & & & & & & & & & & & & & -1.03 & 0.441 \\
\hline CD3g & CD3g & & & & & & & & & & & & $x$ & & 1.04 & 0.447 \\
\hline CD354 & TREM1 & & & & & & & & & $x$ & & & & & 1.04 & 0.470 \\
\hline CD159c & KLRC2 & & & & & & & & & & $x$ & $x$ & & & 1.01 & 0.484 \\
\hline CD161 & KLRB1 & & & & & & & & & & $x$ & & $x$ & & 1.01 & 0.502 \\
\hline CD226 & CD226 & & $x$ & & & & & $x$ & & & $x$ & & $x$ & & -1.01 & 0.504 \\
\hline CD94 & KLRD1 & & & & & & & & & & $x$ & $x$ & & & 1.01 & 0.505 \\
\hline CD69 & CD69 & $x$ & & & & & & $x$ & & & $x$ & & $x$ & & 1.00 & 0.525 \\
\hline CD2 & $\mathrm{CD} 2$ & & & & & & & & & & $x$ & & $x$ & $x$ & 1.15 & 0.074 \\
\hline CD203c & ENPP3 & & & & $x$ & & & & $x$ & & & & & & 1.15 & 0.005 \\
\hline
\end{tabular}

${ }^{1}$ Gene expression fold change (FC), significantly different when Q-value $<0.05$ in paired t-test with Bayesian correction (Limma) during the weight stabilization phase for VLCD ( $\mathrm{n}=26$ ).

${ }^{2}$ Each cross represents in which immune cell a specific surface marker gene is expressed based on http://docs.abcam.com/pdf/immunology/Guide-to-human-CD-antigens.pdf. ADGRE2, adhesion G protein-coupled receptor E2; CD, cluster of differentiation; ENPP3, ectonucleotide pyrophosphatase/phosphodiesterase 3; IGLL1, immunoglobulin lambda-like polypeptide 1; 
ITGAL, integrin, alpha L; ITGAM, integrin, alpha M; ITGAX, integrin, alpha X; KLRB1, killer cell lectin-like receptor subfamily B, member 1; KLRC2, killer cell lectin-like receptor subfamily C, member 2; KLRD1, killer cell lectin-like receptor subfamily D, member 1; LAMP1, lysosomal-associated membrane protein 1; LAMP2, lysosomal-associated membrane protein 2; MSR1, macrophage scavenger receptor 1; NCAM1, neural cell adhesion molecule 1; SIGLEC1, sialic acid binding Ig-like lectin 1; SLAMF6, SLAM family member 6; TLR1, toll-like receptor 1; TREM1, triggering receptor expressed on myeloid cells $1 ; \mathrm{VLCD}$, very-low-calorie diet. 


\section{Chapter 4}

\section{Weight loss-induced stress in subcutaneous adipose tissue is related to weight regain}

Nadia J.T. Roumans, Stefan G. Camps, Johan Renes, Freek G. Bouwman, Klaas R. Westerterp and Edwin C.M. Mariman

British Journal of Nutrition, 2015, 115 (5): 913-920 


\begin{abstract}
Initial successful weight loss is often followed by weight regain after the dietary intervention. Compared with lean people, cellular stress in adipose tissue is increased in obese subjects. However, the relation between cellular stress and the risk for weight regain after weight loss is unclear. Therefore, we determined the expression levels of stress proteins during weight loss and weight maintenance in relation to weight regain. In vivo findings were compared with results from in vitro cultured human Simpson-Golabi-Behmel syndrome (SGBS) adipocytes. In total, eighteen healthy subjects underwent an 8-week diet programme with a 10-month follow-up. Participants were categorised as weight maintainers or weight regainers (WR) depending on their weight changes during the intervention. Abdominal subcutaneous adipose tissue biopsies were obtained before and after the diet and after the follow-up. In vitro differentiated SGBS adipocytes were starved for $96 \mathrm{~h}$ with low $(0.55 \mathrm{mM})$ glucose. Levels of stress proteins were determined by Western blotting. WR showed increased expressions of $\beta$-actin, calnexin, heat shock protein (HSP) 27, HSP60 and HSP70. Changes of $\beta$-actin, HSP27 and HSP70 are linked to HSP60, a proposed key factor in weight regain after weight loss. SGBS adipocytes showed increased levels of $\beta$-actin and HSP60 after $96 \mathrm{~h}$ of glucose restriction. The increased level of cellular stress proteins in the adipose tissue of WR probably resides in the adipocytes as shown by in vitro experiments. Cellular stress accumulated in adipose tissue during weight loss may be a risk factor for weight regain.
\end{abstract}




\section{Introduction}

The prevalence of overweight and obesity has risen in recent years, causing a worldwide public health problem due to an increased risk for the metabolic syndrome and development of type 2 diabetes, CVD and cancer. This risk can be significantly lowered by losing weight (1). Usually, losing weight can be readily achieved, but sustaining the lower weight is problematic. Successfully maintaining weight loss, defined as 'keeping off an intentional loss of at least $10 \%$ body weight for at least one year' (2), is proven to be difficult. In general, up to $80 \%$ of the people are unsuccessful ( 3 , 4). It is therefore of great importance to understand the mechanisms that influence the risk for weight regain. Many studies have already shown the involvement of various psychosocial and lifestyle factors in weight maintenance such as motivation to lose weight, social support, physical activity and eating habits $(3,5)$. Other studies have shown effects of physiological and molecular parameters on weight maintenance - for example, increased insulin sensitivity after weight loss is associated with weight regain $(6,7)$. In addition, fasting insulin and homeostasis model assessment of insulin resistance (HOMA-IR) are associated with weight regain (8). Wang et al. (9) showed that men with the metabolic syndrome at baseline were more at risk for weight regain than men without this condition. Further, it has been shown that a higher protein intake after weight loss improves weight maintenance (10). Irisin levels decrease when body weight is reduced but returns to baseline levels in subjects regaining the lost weight (11). All these findings indicate the involvement of various factors in weight regain or maintenance after weight loss. Studies have pointed at special roles of sex hormones (9), metabolic factors $(9,12)$, hunger and satiety hormones (13) as well as epigenetic modifications such as methylation of the neuropeptide $\mathrm{Y}$ and pro-opiomelanocortin gene promoters in maintenance of weight loss (14). The present study focused particularly on the adipose tissue as a key player for weight regain or maintenance after weight loss.

Baseline BMI, fat mass and plasma leptin concentrations are associated with increased risk for weight regain, indicating an important role for adipocytes $(15,16)$. Mauriège et al. $(17)$ showed that metabolic parameters of the subcutaneous adipose tissue are related to weight regain. Change of lipoprotein lipase (LPL) activity during weight loss was negatively related to weight regain in women, whereas change of alpha 2 adrenergic receptor ( $\alpha 2-A R)$ was positively related to weight regain in men. The latter effect seems to occur despite the fact that adrenaline stimulated lipolysis returns to pre-diet levels during the weight-maintenance phase (18). In a weight loss-maintenance study, Verhoef et al. (19) observed that the change in weight during follow-up was related to a change in the levels of the lipolytic enzyme adipose triglyceride lipase in adipose tissue during weight loss. Compared with lean people, the subcutaneous adipose tissue of obese people shows higher endoplasmic reticulum (ER) stress at the level of proteins and gene expression (20). Sharma et al. (21) reported a positive correlation between BMI and activating transcription factor 67 (ATF67)-induced ER stress markers. Human individuals losing weight after a gastric bypass showed decreased levels of ER stress in the adipose tissue, linking weight changes directly to ER stress (22). Although those findings suggest that cellular stress is a consequence of weight gain, it might well be that cellular stress is also a factor that stimulates accumulation of fat. In fact, reactive oxygen species promote the initiation of adipogenesis as well as the terminal differentiation of adipocytes $(23,24)$. In this regard, we hypothesized that cellular stress of adipocytes could also play a role in the risk for weight regain after weight loss. In order to investigate this, we have compared levels of stress-related proteins in the adipose tissue from overweight men and women during weight loss and follow-up. In addition, we compared in vivo findings with observations from in vitro cultured adipocytes after 
glucose restriction. As stress markers, we selected eight different proteins, which are all involved in different aspects of cellular stress; these were $\beta$-actin, binding Ig protein (BiP), calnexin, heat shock protein (HSP) 27, HSP60, HSP70, superoxide dismutase (SOD) 1 and SOD2. $\beta$-Actin is a component of actin microfilaments that provide structural support and mediate cellular motion (25). BiP is involved in translocation, folding and assembly of secretory and transmembrane proteins within the ER (26).

Calnexin is a Ca-binding protein involved in proper folding of glycoproteins in the ER $(27,28)$. HSP27 activates the proteasome to degrade unnecessary or damaged proteins $(29,30)$, whereas HSP60 acts as a chaperonin for proteins to be transported to the mitochondrion (20). HSP60 is also released because of inflammatory stress to exert autocrine/paracrine effects on adipocytes (31). HSP70 binds to misfolded proteins after stress-induced protein damage (32). SOD 1 and 2 catalyse conversion of superoxide radicals into peroxide and $\mathrm{O} 2$ to defend the cell against oxidative stress (33). These specific proteins have been selected because several studies have already shown that they can be regarded as markers of cellular stress, such as ER stress and oxidative stress $(25-28,31,33)$. Furthermore, based on proteomics observations, Wang et al. (34) have suggested an association between these proteins and cellular stress. Our objective was to test the hypothesis that altered expressions of the above mentioned proteins during weight loss and follow-up are related to the risk for weight regain.

\section{Material and methods}

\section{Subjects and study design}

The eighteen subjects of the present study (nine females, nine males), aged 20-55 years with a BMI of $27-39 \mathrm{~kg} / \mathrm{m}^{2}$, were selected from a larger cohort, which underwent a weight loss-maintenance intervention (19). This study was conducted according to the Declaration of Helsinki guidelines and registered on ClinicalTrials.gov (registration number: NCT01015508). All procedures involving human participants were approved by the Central Committee on Human Research and by the Medical Ethical Committee of Maastricht University, The Netherlands. Written informed consent was obtained from all subjects.

Selection of the eighteen subjects was based on their weight change throughout the intervention. In short, subjects were healthy, non-smoking and not using medication (except for woman using oral contraception). All subjects maintained a stable weight for three months prior to the intervention. Participants followed an 8-week, very-low-energy diet with about $2.1 \mathrm{MJ} /$ day (Modifast, Nutrition et Santé Benelux). The diet provided 50g carbohydrates, $52 \mathrm{~g}$ protein, $7 \mathrm{~g}$ fat and a micronutrient content, which met the Dutch recommended daily intake. Following weight loss, subjects were instructed to maintain their new body weight for a period of 10 months without following a prescribed diet. However, subjects did receive advice on monitoring and limiting food intake. At three time points, before diet (t0), after diet (t2) and after 10 months follow-up (t12), adipose tissue biopsies and plasma samples were obtained. Body weight was measured in underwear after overnight fasting using a calibrated scale of the BodPod ${ }^{\circledR}$.

Test subjects were classified into two groups according to the percentage weight loss during the diet, and percentage weight regain during follow-up: weight maintainers (WM) and weight regainers (WR). Participants were categorized as WM if there was a weight reduction of at least $10 \%$ but then 
regained less than $6 \%$ weight during follow-up. Participants were categorized as WR if there was a weight reduction of at least $10 \%$ but then regained $6 \%$ or more weight.

\section{Adipose tissue biopsies and protein isolation}

Abdominal subcutaneous adipose tissue biopsies were obtained by needle biopsy under local anaesthesia (2\% lidocaine, Fresenius Kabi) after an overnight fast. Tissue was immediately rinsed in saline, frozen in liquid nitrogen and stored at $-80^{\circ} \mathrm{C}$ until protein isolation. About $350 \mathrm{mg}$ of frozen adipose tissue was ground in a mortar with liquid nitrogen. The powder was dissolved in $200 \mu \mathrm{l}$ of 8 $M$ urea, 2\% (w/v) 3-[(3-cholamidopropyl)dimethylammonio]-1-propanesulfonate (CHAPS) and $65 \mathrm{mM}$-dithiothreitol/100 $\mathrm{mg}$ biopsy and vortexed for 5 minutes. The homogenate was centrifuged for 30 minutes $14000 \mathrm{rpm}$ at $10^{\circ} \mathrm{C}$. The supernatant was carefully collected, aliquoted and stored at $80^{\circ} \mathrm{C}$ until Western blotting. Protein concentrations were determined with a Bradfort-based protein assay (Bio-Rad).

\section{In vitro cell culture experiments}

Human Simpson-Golabi-Behmel Syndrome (SGBS) cells were cultured and differentiated as described previously (35). In brief, SGBS preadipocytes were cultured in a T25 flask till $90 \%$ confluence in Dulbecco's modified Eagle's medium: Nutrient Mixture F-12 (DMEM/F12) medium supplemented with $1 \%$ penicillin/streptomycin (Life Technologies), $10 \%$ fetal bovine serum (Bodinco), $66 \mathrm{nM}$ biotin and $33 \mathrm{nM}$ D-pantothenic acid (Sigma-Aldrich). Confluent preadipocytes were split into two 150-mm petri dishes; a starvation dish and a control dish. In parallel, the two dishes were cultured until 80 to $90 \%$ confluence in the same medium as described above. Medium was changed every 2-3 days. To induce differentiation, confluent pre-adipocytes were washed with PBS buffer and medium was changed to serum free DMEM/F12 medium containing $0.5 \mathrm{mM} 3-$ isobutyl-1-methylxanthine (IBMX), $25 \mathrm{nM}$ dexamethasone, $2 \mu \mathrm{M}$ rosiglitazone, $0.01 \mathrm{mg} / \mathrm{mL}$ human transferrin, $20 \mathrm{nM}$ insulin, $100 \mathrm{nM}$ cortisol and $0.2 \mathrm{nM}$ triiodothyronine (Sigma-Aldrich). After 4 days, cells were further cultured in serum free DMEM/F12 medium containing $20 \mathrm{nM}$ insulin, $100 \mathrm{nM}$ cortisol, $0.01 \mathrm{mg} / \mathrm{mL}$ human transferrin, and $0.2 \mathrm{nM}$ triiodothyronine. Every 2 days the medium was refreshed. After 14 days, $65-80 \%$ of the pre-adipocytes were differentiated into mature adipocytes.

For starvation experiments, mature adipocytes were cultured in DMEM/F12 medium without glucose supplemented with $1 \%$ penicillin/streptomycin, $20 \mathrm{nM}$ insulin and $0.55 \mathrm{mM}$ glucose for a period of $96 \mathrm{~h}$ as glucose restriction to lose fat (36). As control, mature adipocytes, originating from the same pre-adipocyte as the starved adipocytes, were cultured in the same medium with $17.5 \mathrm{mM}$ glucose. After $96 \mathrm{~h}$, cells were lysed using RIPA buffer and protein concentrations were determined with the Pierce BCA Protein Assay Kit (Thermo Fischer). Samples were stored at $-80^{\circ} \mathrm{C}$ until Western blotting. This entire procedure was performed three times in parallel to create three separate experiments.

\section{Western blotting}

A total of $15 \mu \mathrm{g}$ of protein from in vitro or in vivo samples was separated on a $12 \%$ SDSpolyacrylamide Criterion gel (Bio-Rad) at $180 \mathrm{~V}$. After electrophoretic separation, proteins were transferred to $0.45 \mu \mathrm{m}$ nitrocellulose membranes in a Trans-Blot Turbo Transfer System ( $30 \mathrm{~min}$ at 25 $\mathrm{V}$, Bio-Rad). Afterwards, the membranes are stained with Ponceau $\mathrm{S}$ to check for protein bands. Following destaining, blots were blocked for 1 hour in Tris-buffered saline containing $0.1 \%$ Tween 20 (TBST) and 5\% non-fat dry milk powder. Thereafter, blots were incubated overnight at $4{ }^{\circ} \mathrm{C}$ with primary antibodies against $\beta$-actin (1:1000 dilution, Santa Cruz), BiP (1:300 dilution, R\&D systems), 
calnexin (1:1000 dilution, Cell signaling), HSP27 (1:1000 dilution, Cell signaling), HSP70 (1:1000 dilution, R\&D systems), SOD1 (1:800 dilution, R\&D systems) and SOD2 (1:1000 dilution, R\&D systems) in TBST containing $5 \%$ non-fat dry milk powder. After incubation with primary antibody, membranes were washed three times for $10 \mathrm{~min}$ with TBST and incubated for $1.5 \mathrm{~h}$ with a 1:10000 dilution of horseradish peroxidase-conjugated secondary antibody (DAKO) in TBST containing 5\% non-fat dry milk powder. After washing, bands were visualised using a chemiluminescent substrate (SuperSignal CL, Thermo Fischer Scientific) and a charge-coupled device (CCD) camera (XRS-system, Bio-Rad). Data were quantified using Image LabTM 4.0 Software (Bio-Rad).

A pooled sample was used to correct for differences between blots. Blots were normalized using all protein bands seen with Ponceau $S$ as a measure of total protein in the sample instead of a housekeeping protein. Generally used housekeeping proteins, such as $\beta$-actin, are influenced by dieting as previously shown in 2D-gel electrophoresis analysis by Bouwman et al. (37), while Ponceau $S$ has recently been indicated to be a suitable alternative for housekeeping proteins (38).

\section{HSP60 plasma concentrations}

Plasma samples were stored at $-80^{\circ} \mathrm{C}$ after withdrawing. HSP60 concentrations were determined by ELISA (Cusabio Biotech) following the manufacturer's instructions. Absorbance was read by a spectrophotometer at $450 \mathrm{~nm}$.

\section{Statistical analyses}

Independent T-test was carried out for baseline comparisons between WM and WR. ANOVA repeated measures were carried out to determine possible differences over time within a group for the human intervention study. For the in vitro measurements, a dependent T-test was carried out to determine differences between control cells and glucose restricted cells. The dependent T-test was used because control cells and glucose starved cells originate from the same cultured pre-adipocytes. For the human study, fold changes during weight loss and during the whole study were evaluated by ratio of the values in t2:t0 and t12:t0 respectively. Fold change comparisons between WM and WR were performed by using independent T-test.

Pearson R's and Spearman Rho's correlation coefficients were calculated for relationships between parameters during the dieting period. Only correlations found significant with both tests were reported, this was done to make the analysis more stringent and reliable. Spearman rank correlation might be the preferred method since we do not a priori know whether the protein changes are in a linear relationship. On the other hand, when proteins are closely functionally interacting, a linear relationship might be expected. Therefore, we decided to select only values that were significant $(\mathrm{P}<0.01)$ with both methods. Statistical analyses were done using SPSS 20.0 for Windows (SPSS Inc.). For all statistical tests $\mathrm{P}<0.05$ was considered to be statistically significant, except for correlations $(\mathrm{P}<0.01)$. Variation in the number of participants between analyses is due to the exclusion of subjects with missing data. All variables were checked for normal distribution, and variables with a skewed distribution were In-transformed to satisfy conditions of normality. Extreme outliers (values higher than $3 x$ Interquartile range calculated with SPSS) influencing the data were removed during statistical analysis. Data are presented as mean \pm SEM, unless otherwise indicated. 


\section{Results}

\section{Subject characteristics}

No significant difference was observed between the WM and WR group for baseline weight and BMI. Body weight was significantly reduced after diet in both groups, and during follow-up weight gain was significant in the WR group $(P<0.001)$, but not in the WM group (Table 1$)$. The WR group decreased weight by $14.3 \%$ and regained $8.2 \%$ of the weight at $t 2$. The WM group decreased weight by $14.4 \%$ and only regained $0.9 \%$. Figure 1 shows individual weight changes of the WM and WR groups.

Table 1: Subject characteristic of weight regainers and weight maintainers at time points t0, t2 and t12.

\begin{tabular}{cccc}
\hline & Study start (t0) & After weight loss (t2) & After follow-up (t12) \\
\hline Weight maintainers & & & \\
Sex (male/female) & $5 / 4$ & $5 / 4$ & $5 / 4$ \\
Weight $(\mathrm{kg}){ }^{2}$ & $95.4 \pm 5.7$ & $81.7 \pm 4.6$ & $82.8 \pm 5.5$ \\
$\mathrm{BMI}\left(\mathrm{kg} / \mathrm{m}^{2}\right)^{\dagger}$ & $32.0 \pm 0.7$ & $27.5 \pm 0.7$ & $27.7 \pm 0.8$ \\
& & \\
Weight regainers & & \\
Sex (male/female) & $4 / 5$ & $4 / 5$ & $4 / 5$ \\
Weight $(\mathrm{kg})+\ddagger$ & $95.4 \pm 5.7$ & $81.8 \pm 5.0$ & $89.1 \pm 5.3$ \\
BMI $\left(\mathrm{kg} / \mathrm{m}^{2}\right)+\ddagger$ & $31.5 \pm 1.1$ & $27.1 \pm 1.1$ & $29.4 \pm 1.1$ \\
\hline Mean values \pm standard errors. Repeated-measures ANOVA between time points: $+\mathrm{P}<0.001$ (t0-t2); $\ddagger \mathrm{P}<0.001$ (t2-t12).
\end{tabular}
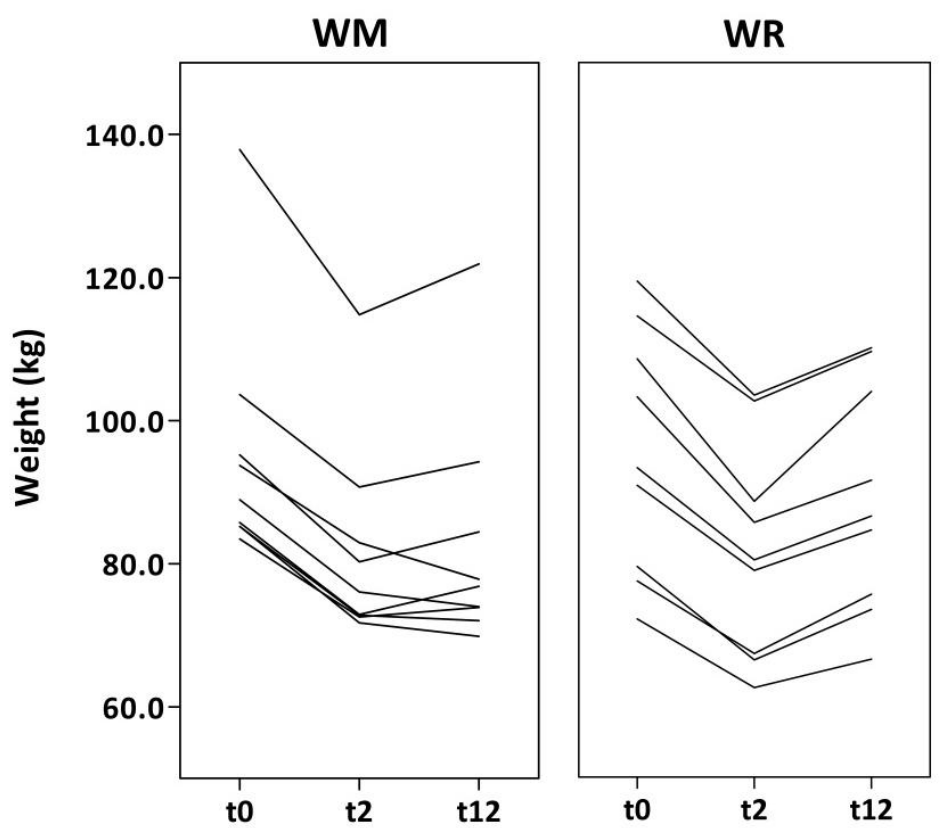

Figure 1: Body weight progression during the course of the study for weight maintainers (WM; $n=9)$ and weight regainers $(W R ; n=9)$. Each line represents the body weight $(\mathrm{kg})$ of an individual measured before 8 weeks of a very-low-energy diet (t0), after the diet (t2), and after 10 months of follow-up (t12).

\section{Stress related proteins in adipose tissue after calorie restriction}

After weight loss, $\beta$-actin, BiP, calnexin, HSP27, HPS60 and HSP70 levels decrease for WM while an increase was observed for WR as shown in Table 2. Fold changes between two time points were calculated and compared between the WR and the WM group (Figure 2). During the weight loss period (t0-t2), the WR group differed from the WM group with respect to $\beta$-actin (trend), calnexin 
(trend), HSP27 (trend), HSP60 and HSP70 (Figure 2A). Levels of stress proteins only changed during weight loss, as during the follow-up period ( $\mathrm{t} 2 \mathrm{t}$ - 12 ) no change for any protein was observed (data not shown). After the complete intervention (t0 - t12), the WR group still differed from the WM group with respect to HSP27 (trend) and HSP70 (Figure 2B). BiP, SOD1 and SOD2 did not significantly change during weight loss. After dieting, no significant difference in plasma HSP60 levels was observed between WR and WM. In addition plasma HSP60 levels did not reflect HSP60 concentrations in adipose tissue (data not shown).

Table 2: Protein abundance levels measured by Western blot at three time points.

\begin{tabular}{|c|c|c|c|}
\hline & Study start (t0) & After weight loss (t2) & After follow-up (t12) \\
\hline \multicolumn{4}{|l|}{ B-actin } \\
\hline WM & $3.93 \pm 0.58$ & $3.51 \pm 0.48$ & $3.72 \pm 0.56$ \\
\hline WR & $4.03 \pm 0.51$ & $4.93 \pm 0.45$ & $4.27 \pm 0.46$ \\
\hline \multicolumn{4}{|l|}{ BiP } \\
\hline$W M+$ & $3.82 \pm 1.09$ & $1.27 \pm 0.60$ & $1.92 \pm 0.47$ \\
\hline$W R \mp \S$ & $1.54 \pm 0.54$ & $2.12 \pm 0.69$ & $3.59 \pm 0.53$ \\
\hline \multicolumn{4}{|l|}{ Calnexin } \\
\hline WM & $2.88 \pm 1.26$ & $0.76 \pm 0.28$ & $1.20 \pm 0.51$ \\
\hline WR & $1.36 \pm 0.58$ & $3.62 \pm 1.38$ & $1.73 \pm 0.96$ \\
\hline \multicolumn{4}{|l|}{$H S P 27$} \\
\hline$W M+$ & $3.46 \pm 1.25$ & $2.60 \pm 1.88$ & $1.58 \pm 0.81$ \\
\hline WR & $2.56 \pm 1.50$ & $5.80 \pm 2.44$ & $2.98 \pm 1.22$ \\
\hline \multicolumn{4}{|l|}{ HSP6O } \\
\hline WM II & $4.40 \pm 1.34$ & $1.46 \pm 0.60$ & $2.87 \pm 0.78$ \\
\hline WR & $2.80 \pm 0.54$ & $3.66 \pm 0.67$ & $3.92 \pm 0.79$ \\
\hline \multicolumn{4}{|l|}{ HSP7O } \\
\hline WM & $2.77 \pm 0.68$ & $2.30 \pm 1.00$ & $2.48 \pm 0.38$ \\
\hline WR II § & $2.17 \pm 0.55$ & $3.85 \pm 0.90$ & $4.50 \pm 0.72$ \\
\hline \multicolumn{4}{|l|}{ SOD1 } \\
\hline WM & $1.04 \pm 0.29$ & $1.57 \pm 0.49$ & $1.17 \pm 0.29$ \\
\hline WR & $0.96 \pm 0.43$ & $1.40 \pm 0.41$ & $1.12 \pm 0.40$ \\
\hline \multicolumn{4}{|l|}{ SOD2 } \\
\hline WM & $1.17 \pm 0.42$ & $1.64 \pm 0.94$ & $1.22 \pm 0.51$ \\
\hline WR & $1.71 \pm 0.60$ & $2.51 \pm 0.63$ & $1.66 \pm 0.61$ \\
\hline
\end{tabular}

Mean values \pm standard errors. Repeated-measures ANOVA between time points: $+\mathrm{P}>0.05-\mathrm{P}<0.1$ (t0-t2); $¥ \mathrm{P} \leq 0.05$ (t2-t12); $\S \mathrm{P} \leq 0.05$ (t0-t12); II $\mathrm{P} \leq 0.05$ (t0-t2). WM, weight maintainers; WR, weight regainers; BiP, binding Ig protein; HSP, heat shock protein; SOD, superoxide dismutase. 

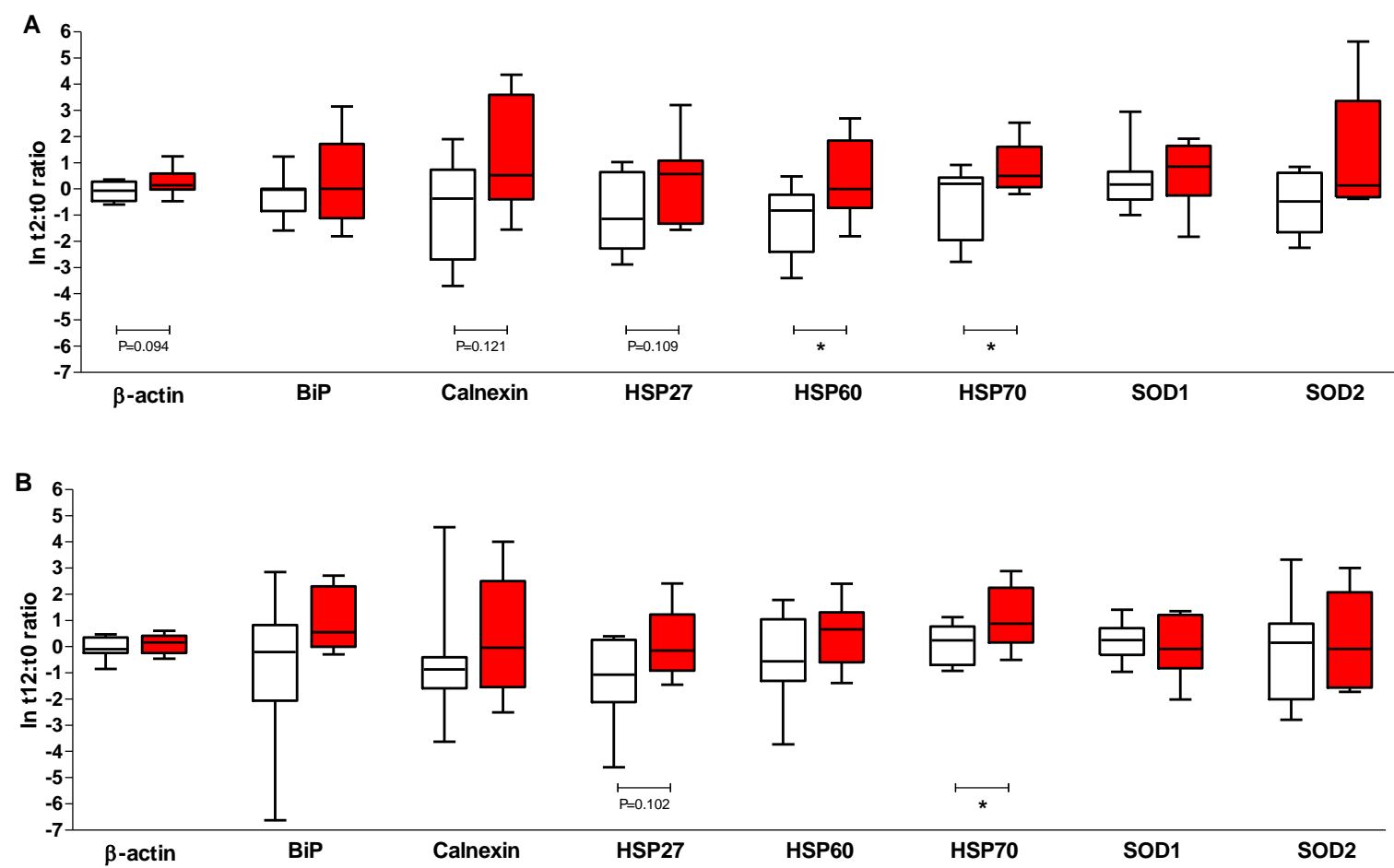

Figure 2: Fold changes in stress-related proteins during (A) the weight loss-phase (after 8 weeks of weight loss (t2) : baseline (t0)) and (B) the whole study (after 10 months of follow-up (t12) : t0). Each box plot shows the median and interquartile range without outliers of the fold change in each protein. Weight maintainers $(\square ; n=9)$ and weight regainers $(\square ; n=9)$. The difference between the two groups was analysed by independent $T$ test on In-transformed values: ${ }^{*} P \leq 0.05$. $\mathrm{BiP}$, binding Ig protein; $\mathrm{HSP}$, heat shock protein; SOD, superoxide dismutase.

During the weight loss phase, changes in stress proteins were correlated in both groups to be able to gain more insight into the mechanistic regulation of weight loss-induced cellular stress. In Supplement Figure 1 the correlation plots can be seen. Based on the significant correlations, we have drawn interaction maps for the WM and the WR group (Figure 3). In both groups the similar regulation of HSP60 with HSP70 is obvious, but whereas in the WM group a link between $\beta$-actin and HSP27 exists, in the WR group these proteins seem to follow the regulation of HSP60. 


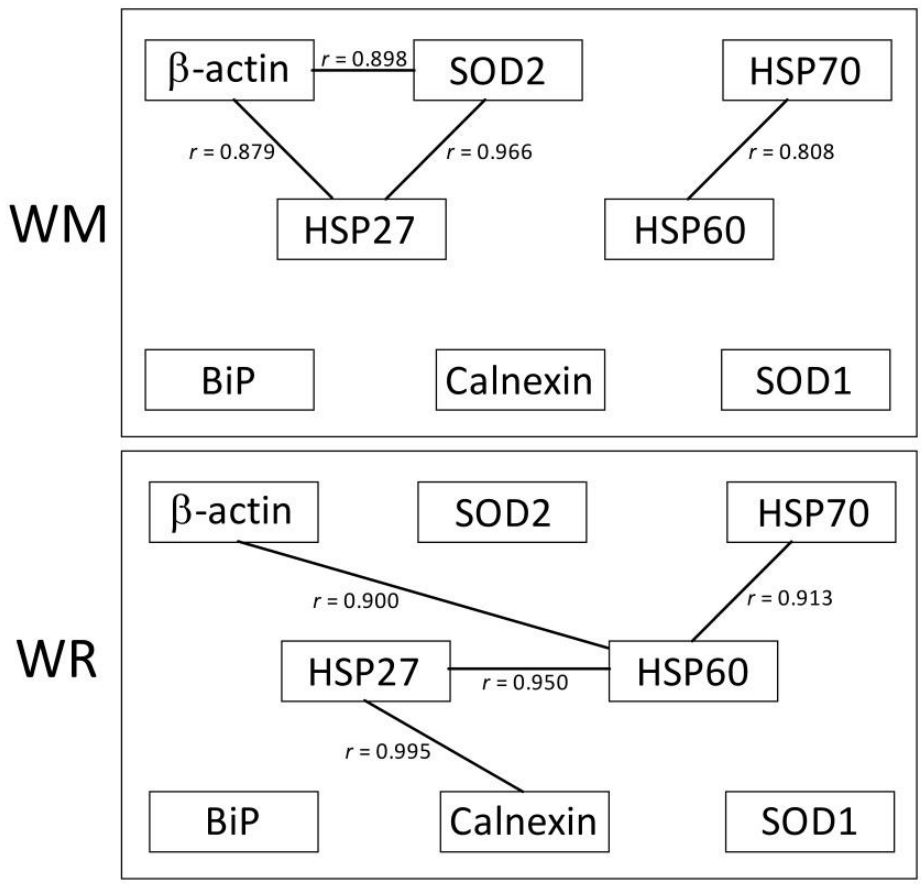

Figure 3: Schematic overview of the correlated proteins within the weight maintainers (WM) and weight regainers (WR) groups during the diet phase ( $\mathrm{t} 0$ - $\mathrm{t} 2)$. Significant correlations $(\mathrm{P} \leq 0.01)$ found with both Pearson $\mathrm{R}$ and Spearman $\mathrm{P}$ are represented by the connecting lines with the correlation coefficients $(r)$. BiP, binding Ig protein; HSP, heat shock protein; SOD, superoxide dismutase.

\section{Stress related proteins in adipocytes after low-glucose starvation}

The in vivo results were based on adipose tissue biopsies, which in addition to adipocytes also contain stromal vascular cells. To investigate whether there is an adipocyte specific stress response to calorie restriction, we performed an in vitro experiment. Mature SGBS adipocytes received glucose restricted medium for 4 days causing around 17\% loss of triglyceride content from the cells as previously reported by Renes et al. (36). In parallel, mature SGBS adipocytes cultured with control medium did not show loss of triglycerides. Cells were then harvested and proteins were isolated. The relative amount of the eight proteins was measured but SOD1 expression appeared to be too low to measure. Similar to the WR group, expression of all measured stress proteins was increased during caloric restriction with statistical significance for $\beta$-actin and HSP60 (Figure 4).

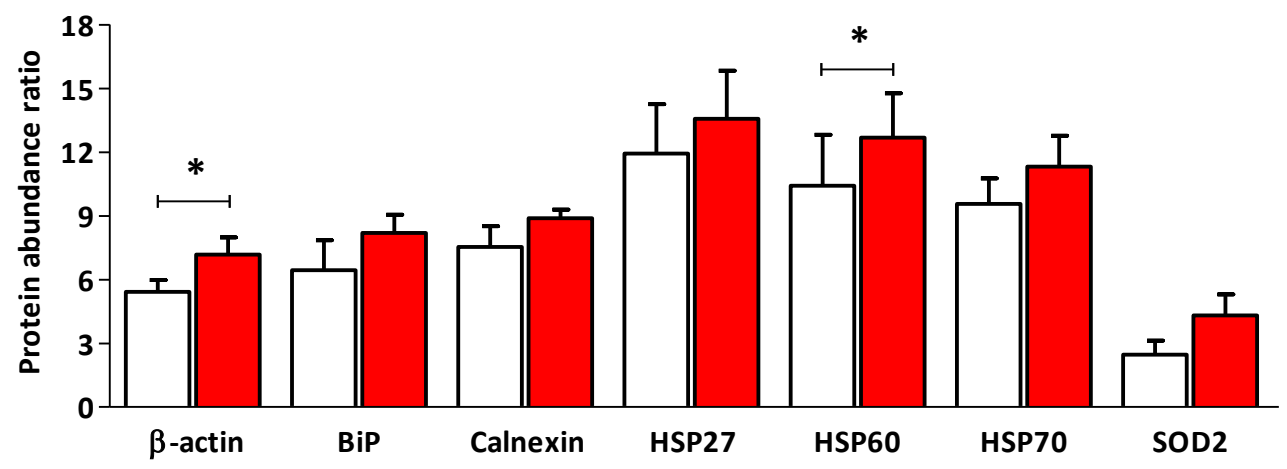

Figure 4: Stress protein levels of SGBS adipocytes after glucose restriction measured with Western blotting. Glucose restriction medium containing $20 \mathrm{nM}$ insulin and $0.55 \mathrm{mM}$ glucose ( $\square$ ). The control group received medium containing 20 $\mathrm{nM}$ insulin and $17.5 \mathrm{mM}$ glucose $(\square)$. All groups consist of $\mathrm{n}=3$ measured in duplicate. Values are means with standard errors. The difference over time was analysed by dependent $\mathrm{T}$ test: ${ }^{*} \mathrm{P} \leq 0.05$. BiP, binding Ig protein; HSP, heat shock protein; SOD, superoxide dismutase. 


\section{Discussion}

The current study results suggest that adipocyte stress is a biological risk factor for weight regain after weight loss. In the WR group the levels of $\beta$-actin, calnexin, HSP27, HSP60 and HSP70 were increased after weight loss compared to the WM group. Correlation analysis indicated that changes of $\beta$-actin, HSP70 and HSP27 are linked to changes in HSP60 as a possible key-factor contributing to weight regain. Increased levels of $\beta$-actin and HSP60 were also observed after 4 days of glucose restriction of SGBS adipocytes indicating that the in vivo observations reside in the mature adipocytes of the adipose tissue.

Our findings show that during weight loss stress proteins increase in the adipose tissue of individuals who are at risk for weight regain. One of those is $\beta$-actin, a component of actin filaments (25). During weight loss adipocytes change shape due to shrinking, which requires re-allocation of cellular components. When parts of the cell need to be moved, 10-30 actin filaments assemble into so-called stress-fibers, which can perform mechanical traction. Accordingly, in our in vitro experiment with cultured SGBS adipocytes, we observed a significant up-regulation of $\beta$-actin after glucose restriction in parallel to the loss of triglycerides and shrinking of the cells. In the adipose tissue of the WR subjects after weight loss, we observed an up-regulation of $\beta$-actin indicating that more stress-fibers are formed and more mechanical stress exists in the adipocytes of the WR group than in those of the WM group. Unfortunately, we were unable to quantify stress-fibers directly. Nevertheless, our $\beta$ actin results are in line with those of Mutch et al. who detected an up-regulation of $\beta$-actin gene expression in WR and a downregulation in WM (39).

HSP27 showed a trend for change in adipose tissue after weight loss and there was no change after glucose restriction in the in vitro cultured adipocytes. On the other hand, in the WM group we found a strong correlation between HSP27 and $\beta$-actin suggesting a functional link between these proteins. It has been shown that HSP27 binds to actin filaments (F-actin) and that under cell stress HSP27 becomes phosphorylated, which enhances binding affinity for F-actin (40). In this regard, HSP27 appears to be involved in the regulation of actin filament dynamics. In renal cells, HSP27 provides protection against the consequences of ATP-depletion and this function was dependent on HSP70 (41), which is known to form complexes with HSP60 (42). In the present study, our correlation map of the WR group showed a link between changes over the diet period for $\beta$-actin, HSP27 and HSP70 via HSP60 (Figure 3). Altogether, these data in combination with our in vitro response to energy depletion, strongly suggest that weight regain relates to cell stress and involves the regulation of actin filament dynamics.

Calnexin increased in the adipose tissue in the WR group after weight loss, whereas in the other group the level seemed to drop. Calnexin retains newly synthesized $\mathrm{N}$-glycosylated proteins inside the ER to ensure proper folding with the help of folding factor ERp57 $(27,28)$. Wrongly folded proteins may enter into a cycle of unfolding and refolding or may be broken down by the ERassociated degradation pathway (43), whereas correctly folded glycoproteins are transported out of the cell. Interestingly, $\mathrm{N}$-glycoproteins are important for the formation of the extracellular matrix (ECM) of adipocytes (44). Our results show relatively high levels of calnexin after dieting in the WR group which seems to be in line with the up-regulation of genes with a focal adhesion function in the WR group of the study of Mutch et al. (39), and their down-regulation in the WM group. 
Besides mechanical stress, other forms of cellular stress might be involved in the risk for weight regain after weight loss. HSP70 is a marker for ER stress, although it may also have a protective function against ER stress-induced apoptosis (45), possibly by inhibiting key stress kinases (46). Our results show increased concentrations of HSP70 after dieting in the WR group. This implies that ER stress is present in the adipose tissue of those subjects and as such might be related to the risk for weight regain after weight loss.

In this study, we found that the WR group had elevated levels of HSP60 in the adipose tissue after dieting compared to WM subjects. HSP60 is present in the circulation of people with type 2 diabetes (47) and increased levels are observed in the adipose tissue of obese subjects compared to lean subjects (20). In starved 3T3-L1 adipocytes an up-regulation of HSP60 is shown compared to nonstarved cells (48), which complies with our present in vitro observations in human SGBS adipocytes. HSP60 stimulates the release of pro-inflammatory adipokines from the adipose tissue promoting inflammation and, as such, may support the development of insulin resistance. This suggests that WR are at higher risk for obesity-related complications. Interestingly, as HSP60 is a chaperone for mitochondrial proteins, an increase in HSP60 after weight loss indicates a dysregulation of the mitochondrial metabolic processes in WR-subjects. However, a possible role for such impairment in the risk of weight regain requires further investigation.

A limitation of the present study is the relatively small number of participants. In this group, the age varied between $20-55$ years and the BMI between $27-39 \mathrm{~kg} / \mathrm{m}^{2}$. Thus, it might be that we included pre- and post-menopausal woman as well as overweight and obese individuals. Nevertheless, this study produced new insights which can form the basis for further studies in larger cohorts. For the moment, it is unclear why some people show increased adipose cellular stress during weight loss while others do not. One explanation might be genetic predisposition. Alternatively, adipocyte size at baseline might play a role. A general model for accumulation of cellular stress during weight loss states that when adipocytes lose fat and shrink, the surrounding ECM is unable to follow the size reduction (49-51). Consequently, mechanical-type cellular stress rises, which can be neutralized most easily by renewed lipid uptake and storage. Indeed, measuring the adipocyte molecular metabolism by using proteomics technologies indicates that after returning to energy balance, adipocytes rapidly prepare for re-storing triglycerides $(37,52)$. Moreover, during weight loss, the plasma leptin level decreases dramatically, out of proportion with the loss of fat mass, which implies that, after weight loss, an extra impulse is given to energy intake to warrant the re-storing of fat and release of cellular stress (53).

In conclusion, analyses of subcutaneous adipose tissue show increased cellular stress after an 8-week diet in a group of subjects who regained most of their weight during follow-up. In vitro cultured mature adipocytes subjected to energy restriction showed similar protein changes. These findings support the idea that adipocyte stress plays a role as a biological risk factor for weight regain after weight loss and suggest involvement of mechanical stress with dynamics of stress fibres. 


\section{References}

1. Bessesen DH. Update on obesity. J Clin Endocrinol Metab, 2008. 93(6):2027-34.

2. Wing RR, Hill JO. Successful weight loss maintenance. Annu Rev Nutr, 2001. 21:323-41.

3. Wu T, Gao X, Chen M, van Dam RM. Long-term effectiveness of diet-plus-exercise interventions vs. diet-only interventions for weight loss: a meta-analysis. Obes Rev, 2009. 10(3):313-23.

4. Barte JC, ter Bogt NC, Bogers RP, Teixeira PJ, Blissmer B, Mori TA, Bemelmans WJ. Maintenance of weight loss after lifestyle interventions for overweight and obesity, a systematic review. Obes Rev, 2010. 11(12):899-906.

5. Elfhag $\mathrm{K}$, Rossner $\mathrm{S}$. Who succeeds in maintaining weight loss? A conceptual review of factors associated with weight loss maintenance and weight regain. Obes Rev, 2005. 6(1):67-85.

6. Yost TJ, Jensen DR, Eckel RH. Weight regain following sustained weight reduction is predicted by relative insulin sensitivity. Obes Res, 1995. 3(6):583-7.

7. Wing RR. Insulin sensitivity as a predictor of weight regain. Obes Res, 1997. 5(1):24-9.

8. Wong $\mathrm{MH}$, Holst $\mathrm{C}$, Astrup A, Handjieva-Darlenska T, Jebb SA, Kafatos A et al. Caloric restriction induces changes in insulin and body weight measurements that are inversely associated with subsequent weight regain. PLoS One, 2012. 7(8):e42858.

9. Wang P, Menheere PP, Astrup A, Andersen MR, van Baak MA, Larsen TM et al. Metabolic syndrome, circulating RBP4, testosterone, and SHBG predict weight regain at 6 months after weight loss in men. Obesity (Silver Spring), 2013. 21(10):1997-2006.

10. Aller EE, Larsen TM, Claus H, Lindroos AK, Kafatos A, Pfeiffer A et al. Weight loss maintenance in overweight subjects on ad libitum diets with high or low protein content and glycemic index: the DIOGENES trial 12-month results. Int J Obes (Lond), 2014. 38(12):1511-7.

11. Crujeiras AB, Pardo M, Arturo RR, Navas-Carretero S, Zulet MA, Martinez JA, Casanueva FF. Longitudinal variation of circulating irisin after an energy restriction-induced weight loss and following weight regain in obese men and women. Am J Hum Biol, 2014. 26(2):198-207.

12. Wang $\mathrm{P}$, Holst C, Wodzig WK, Andersen MR, Astrup A, van Baak MA et al. Circulating ACE is a predictor of weight loss maintenance not only in overweight and obese women, but also in men. Int J Obes (Lond), 2012. 36(12):1545-51.

13. Crujeiras AB, Goyenechea E, Abete I, Lage M, Carreira MC, Martinez JA, Casanueva FF. Weight regain after a diet-induced loss is predicted by higher baseline leptin and lower ghrelin plasma levels. $J$ Clin Endocrinol Metab, 2010. 95(11):5037-44.

14. Crujeiras AB, Campion J, Diaz-Lagares A, Milagro FI, Goyenechea E, Abete I, Casanueva FF, Martinez JA. Association of weight regain with specific methylation levels in the NPY and POMC promoters in leukocytes of obese men: a translational study. Regul Pept, 2013. 186:1-6.

15. Vogels N, Diepvens K, Westerterp-Plantenga MS. Predictors of long-term weight maintenance. Obes Res, 2005. 13(12):2162-8.

16. Vogels N, Westerterp-Plantenga MS. Successful long-term weight maintenance: a 2-year follow-up. Obesity (Silver Spring), 2007. 15(5):1258-66.

17. Mauriege $P$, Imbeault $P$, Doucet E, Lacaille M, Langin D, Almeras N, Despres JP, Tremblay A. Weight loss and regain in obese individuals: a link with adipose tissue metabolism indices? Journal of physiology and biochemistry, 2013. 69(3):497-505.

18. Koppo K, Siklova-Vitkova M, Klimcakova E, Polak J, Marques MA, Berlan M et al. Catecholamine and insulin control of lipolysis in subcutaneous adipose tissue during long-term diet-induced weight loss in obese women. Am J Physiol Endocrinol Metab, 2012. 302(2):E226-32.

19. Verhoef SP, Camps SG, Bouwman FG, Mariman EC, Westerterp KR. Physiological response of adipocytes to weight loss and maintenance. PLoS One, 2013. 8(3):e58011.

20. Boden G, Duan X, Homko C, Molina EJ, Song W, Perez O, Cheung P, Merali S. Increase in endoplasmic reticulum stress-related proteins and genes in adipose tissue of obese, insulin-resistant individuals. Diabetes, 2008. 57(9):2438-44.

21. Sharma NK, Das SK, Mondal AK, Hackney OG, Chu WS, Kern PA, Rasouli N, Spencer HJ, YaoBorengasser A, Elbein SC. Endoplasmic reticulum stress markers are associated with obesity in nondiabetic subjects. J Clin Endocrinol Metab, 2008. 93(11):4532-41.

22. Gregor MF, Yang L, Fabbrini E, Mohammed BS, Eagon JC, Hotamisligil GS, Klein S. Endoplasmic reticulum stress is reduced in tissues of obese subjects after weight loss. Diabetes, 2009. 58(3):693700. 
23. Krautbauer S, Eisinger K, Hader Y, Neumeier M, Buechler C. Manganese superoxide dismutase knockdown in 3T3-L1 preadipocytes impairs subsequent adipogenesis. Mol Cell Biochem, 2014. 393(1-2):6976.

24. Liu GS, Chan EC, Higuchi M, Dusting GJ, Jiang F. Redox mechanisms in regulation of adipocyte differentiation: beyond a general stress response. Cells, 2012. 1(4):976-93.

25. Shawlot W, Deng JM, Fohn LE, Behringer RR. Restricted beta-galactosidase expression of a hygromycin-lacZ gene targeted to the beta-actin locus and embryonic lethality of beta-actin mutant mice. Transgenic Res, 1998. 7(2):95-103.

26. Kleizen B, Braakman I. Protein folding and quality control in the endoplasmic reticulum. Curr Opin Cell Biol, 2004. 16(4):343-9.

27. Guerin R, Arseneault G, Dumont S, Rokeach LA. Calnexin is involved in apoptosis induced by endoplasmic reticulum stress in the fission yeast. Mol Biol Cell, 2008. 19(10):4404-20.

28. Bousette $\mathrm{N}$, Abbasi $\mathrm{C}$, Chis R, Gramolini AO. Calnexin silencing in mouse neonatal cardiomyocytes induces Ca2+ cycling defects, ER stress, and apoptosis. J Cell Physiol, 2014. 229(3):374-83.

29. Garrido C. Size matters: of the small HSP27 and its large oligomers. Cell Death Differ, 2002. 9(5):483-5.

30. Horman S, Galand P, Mosselmans R, Legros N, Leclercq G, Mairesse N. Changes in the phosphorylation status of the $27 \mathrm{kDa}$ heat shock protein (HSP27) associated with the modulation of growth and/or differentiation in MCF-7 cells. Cell Prolif, 1997. 30(1):21-35.

31. Marker T, Sell H, Zillessen P, Glode A, Kriebel J, Ouwens DM et al. Heat shock protein 60 as a mediator of adipose tissue inflammation and insulin resistance. Diabetes, 2012. 61(3):615-25.

32. Mayer RJ, Ciechanover AJ, Rechsteiner M. Protein degradation. Weinheim: Wiley-VCH, 2005.

33. Toichi K, Yamanaka K, Furukawa Y. Disulfide scrambling describes the oligomer formation of superoxide dismutase (SOD1) proteins in the familial form of amyotrophic lateral sclerosis. J Biol Chem, 2013. 288(7):4970-80.

34. Wang P, Bouwman FG, Mariman EC. Generally detected proteins in comparative proteomics--a matter of cellular stress response? Proteomics, 2009. 9(11):2955-66.

35. Wabitsch M, Brenner RE, Melzner I, Braun M, Moller P, Heinze E, Debatin KM, Hauner H. Characterization of a human preadipocyte cell strain with high capacity for adipose differentiation. Int J Obes Relat Metab Disord, 2001. 25(1):8-15.

36. Renes J, Rosenow A, Roumans N, Noben JP, Mariman EC. Calorie restriction-induced changes in the secretome of human adipocytes, comparison with resveratrol-induced secretome effects. Biochim Biophys Acta, 2014.

37. Bouwman FG, Claessens M, van Baak MA, Noben JP, Wang P, Saris WH, Mariman EC. The physiologic effects of caloric restriction are reflected in the in vivo adipocyte-enriched proteome of overweight/obese subjects. J Proteome Res, 2009. 8(12):5532-40.

38. Gilda JE, Gomes AV. Stain-Free total protein staining is a superior loading control to beta-actin for Western blots. Anal Biochem, 2013. 440(2):186-8.

39. Mutch DM, Pers TH, Temanni MR, Pelloux V, Marquez-Quinones A, Holst C et al. A distinct adipose tissue gene expression response to caloric restriction predicts 6-mo weight maintenance in obese subjects. Am J Clin Nutr, 2011. 94(6):1399-409.

40. Clarke JP, Mearow KM. Cell stress promotes the association of phosphorylated HspB1 with F-actin. PLoS One, 2013. 8(7):e68978.

41. Sreedharan R, Riordan M, Thullin G, Van Why S, Siegel NJ, Kashgarian M. The maximal cytoprotective function of the heat shock protein 27 is dependent on heat shock protein 70. Biochim Biophys Acta, 2011. 1813(1):129-35.

42. Sarto C, Binz PA, Mocarelli P. Heat shock proteins in human cancer. Electrophoresis, 2000. 21(6):121826.

43. Helenius A, Aebi M. Intracellular functions of N-linked glycans. Science, 2001. 291(5512):2364-9.

44. Jones FS, Jones PL. The tenascin family of ECM glycoproteins: structure, function, and regulation during embryonic development and tissue remodeling. Dev Dyn, 2000. 218(2):235-59.

45. Mosser DD, Caron AW, Bourget L, Meriin AB, Sherman MY, Morimoto RI, Massie B. The chaperone function of hsp70 is required for protection against stress-induced apoptosis. Mol Cell Biol, 2000. 20(19):7146-59.

46. Simar D, Jacques A, Caillaud C. Heat shock proteins induction reduces stress kinases activation, potentially improving insulin signalling in monocytes from obese subjects. Cell Stress Chaperones, 2012. 17(5):615-21. 
47. Dasu MR, Devaraj S, Park S, Jialal I. Increased toll-like receptor (TLR) activation and TLR ligands in recently diagnosed type 2 diabetic subjects. Diabetes Care, 2010. 33(4):861-8.

48. Renes J, Bouwman F, Noben JP, Evelo C, Robben J, Mariman E. Protein profiling of 3T3-L1 adipocyte differentiation and (tumor necrosis factor alpha-mediated) starvation. Cell Mol Life Sci, 2005. 62(4):492-503.

49. Kolehmainen M, Salopuro T, Schwab US, Kekalainen J, Kallio P, Laaksonen DE et al. Weight reduction modulates expression of genes involved in extracellular matrix and cell death: the GENOBIN study. Int J Obes (Lond), 2008. 32(2):292-303.

50. Mariman EC. Human biology of weight maintenance after weight loss. J Nutrigenet Nutrigenomics, 2012. 5(1):13-25.

51. Mariman EC. An adipobiological model for weight regain after weight loss. Adipobiology, 2011. 3(1):915.

52. Bouwman FG, Wang $P$, van Baak M, Saris WH, Mariman EC. Increased beta-oxidation with improved glucose uptake capacity in adipose tissue from obese after weight loss and maintenance. Obesity (Silver Spring), 2014. 22(3):819-27.

53. Maffei M, Halaas J, Ravussin E, Pratley RE, Lee GH, Zhang Y et al. Leptin levels in human and rodent: measurement of plasma leptin and ob RNA in obese and weight-reduced subjects. Nat Med, 1995. 1(11):1155-61. 
Chapter 4

Supplementary Figures and Tables 
Supplement Figure 1: Correlation plots of fold changes of stress proteins in the weight maintainers (A-D) and weight regainers $(E-H)$ group during the diet phase (t0-t2). Correlation of HSP70 with HSP60 for WM $n=9(A), \beta$-actin with HSP27 for WM $n=8$ (B), $\beta$-actin with SOD2 for WM $n=8$ (C), HSP27 with SOD2 for WM n=7 (D), HSP70 with HSP60 for WR $n=9$ (E), $\beta$ actin with HSP60 for WR n=9 (F), HSP27 with HSP60 for WR $n=7$ (G), HSP27 with Calnexin for WR $n=4(H)$. HSP, heat shock protein; SOD, superoxide dismutase; WR, weight regainers; WM, weight maintainers.
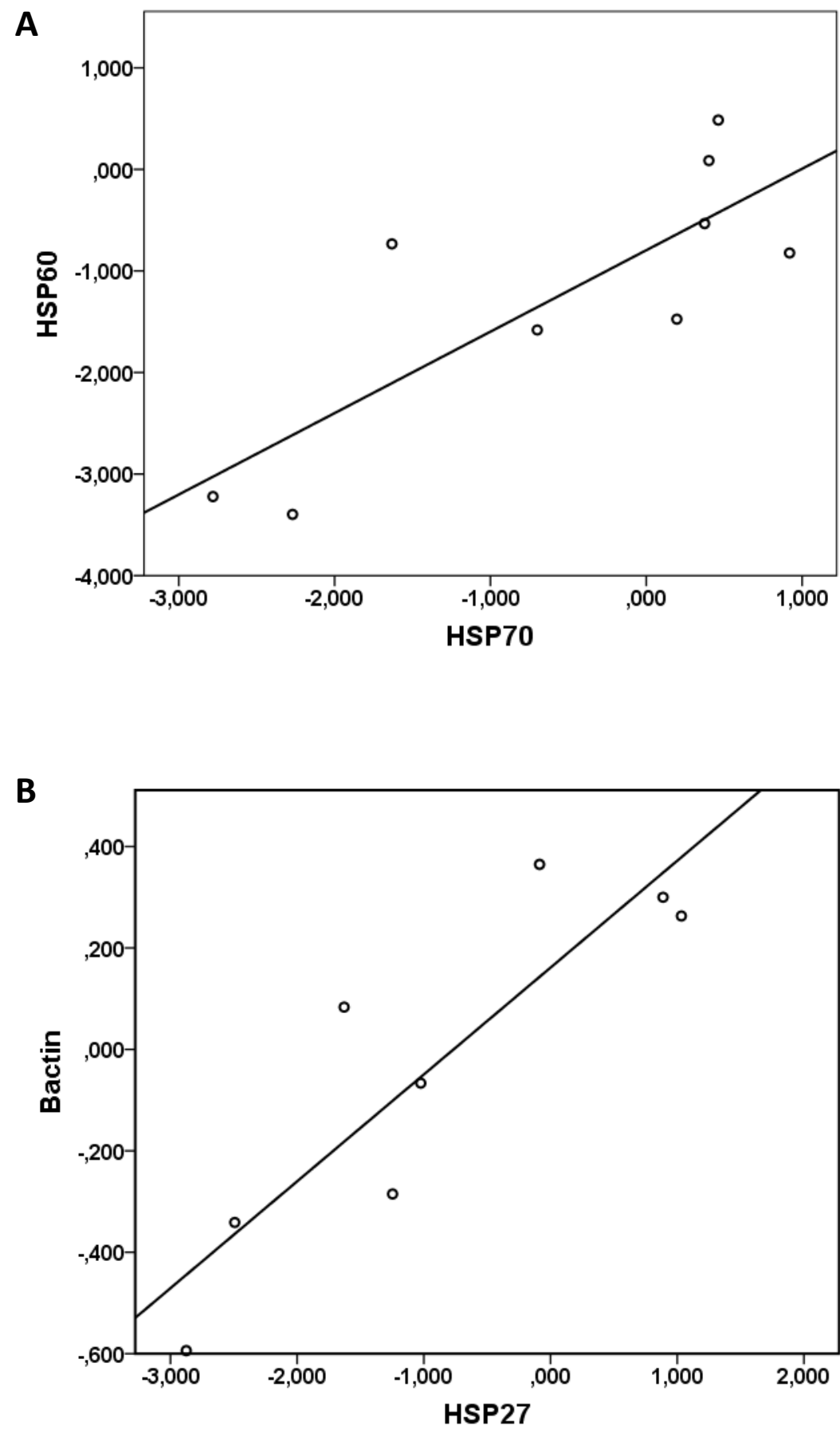
Chapter 4
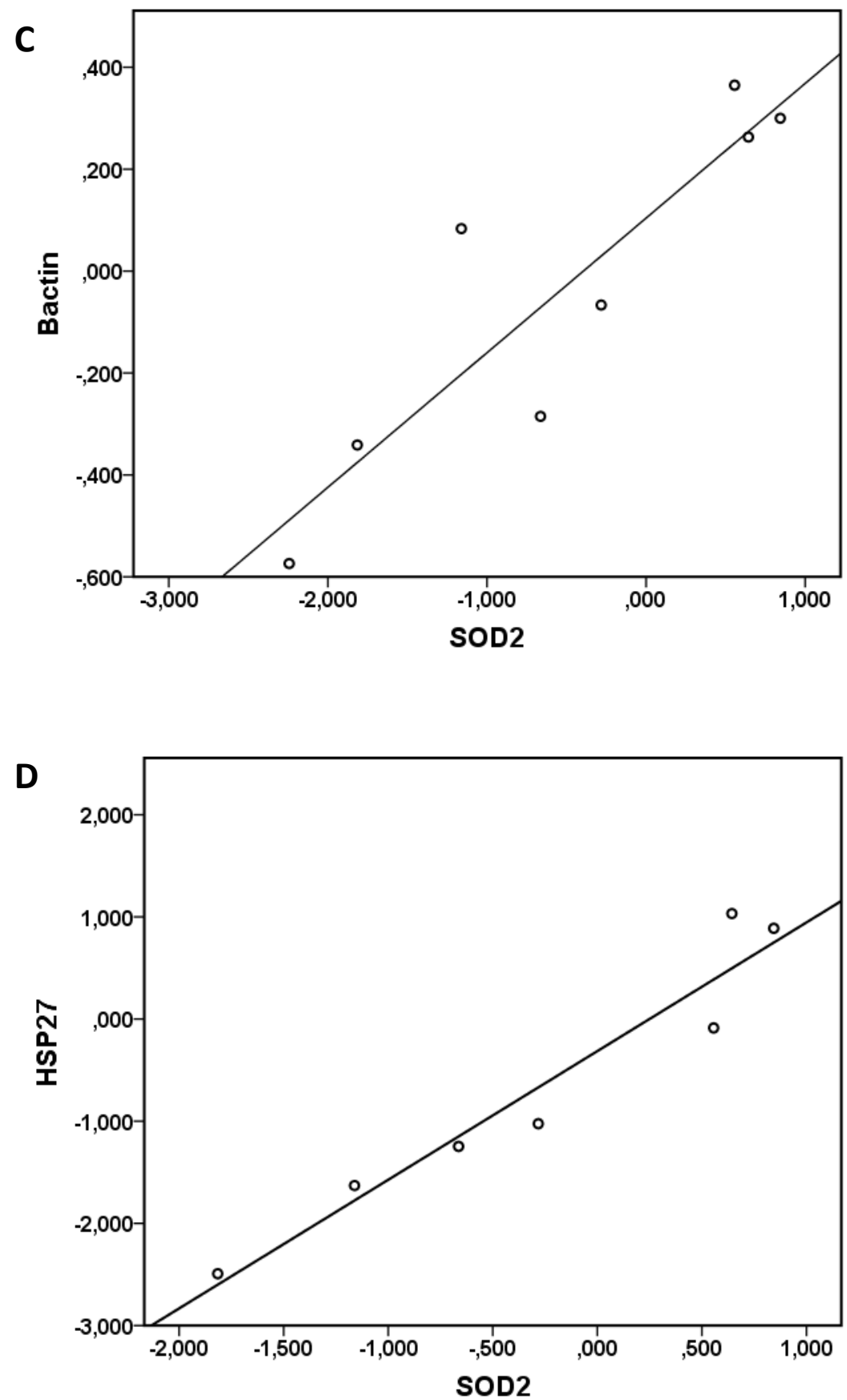

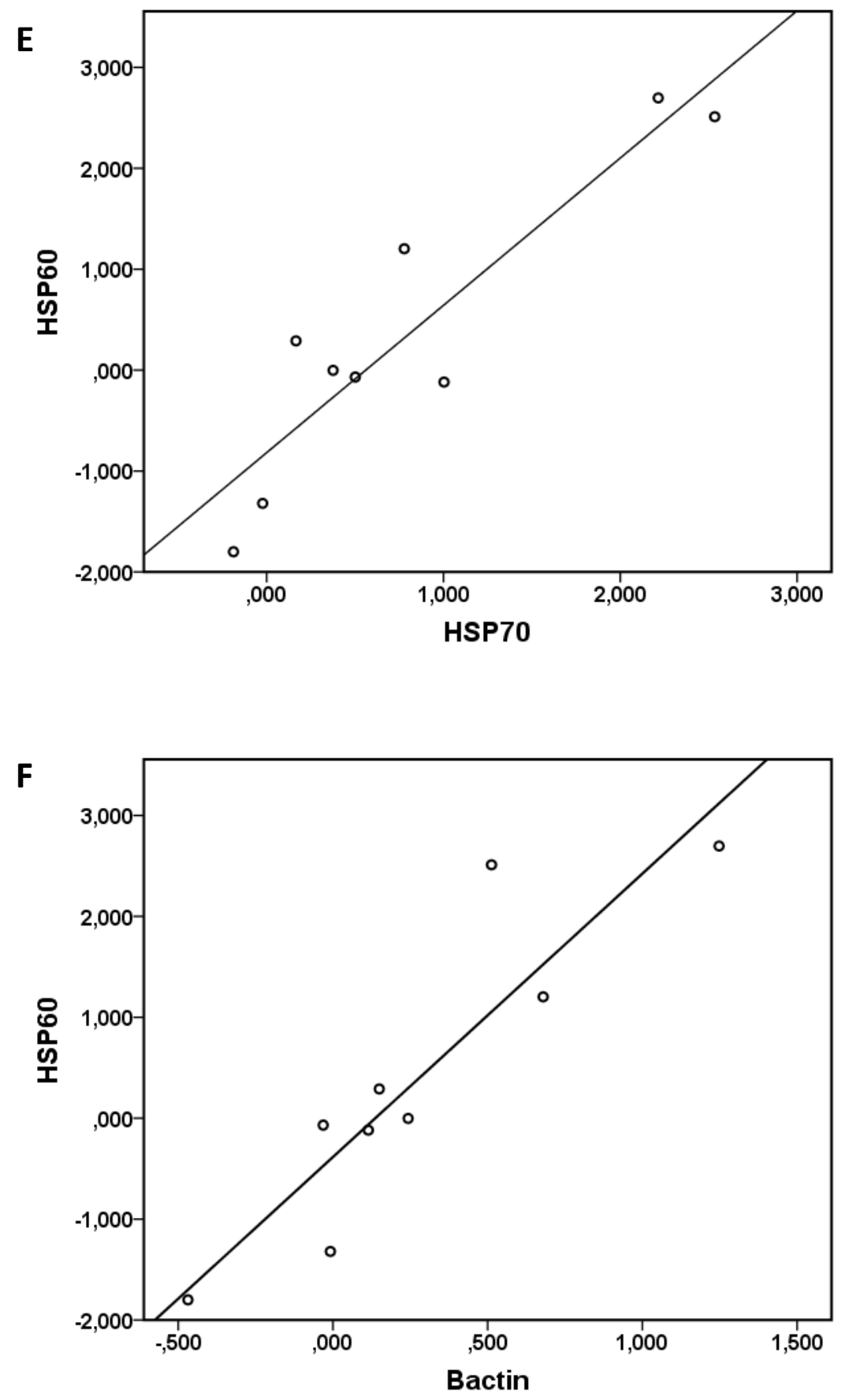
Chapter 4
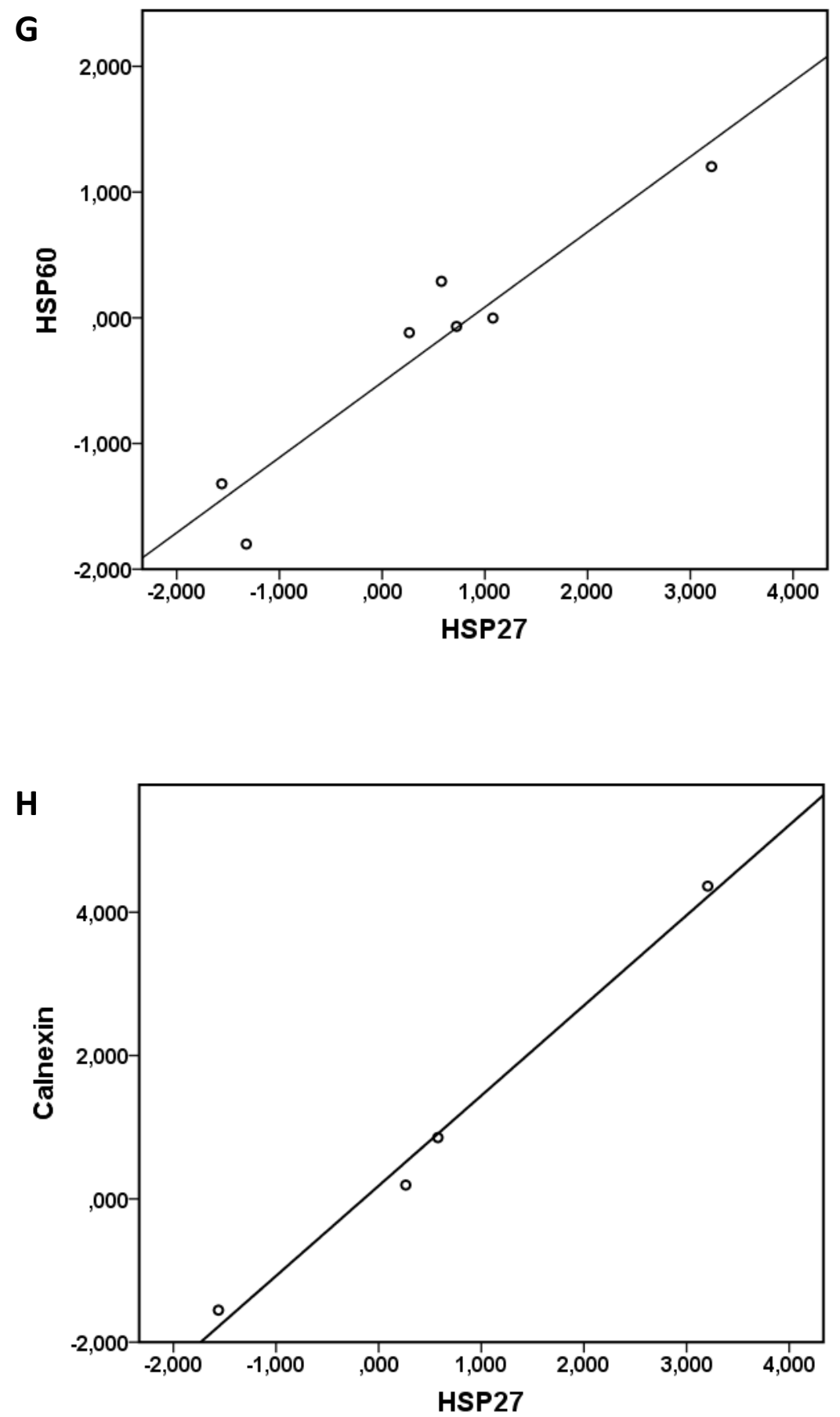


\section{Chapter 5}

\section{Weight loss-induced cellular stress in subcutaneous adipose tissue and the risk for weight regain in overweight and obese adults}




\begin{abstract}
Background/Objective: Weight loss is often followed by weight regain after the dietary intervention (DI). Cellular stress is increased in adipose tissue of obese individuals. However, the relation between cellular stress and weight regain is unclear. Previously, we observed increased adipose tissue cellular stress of participants regaining weight compared to participants maintaining weight loss. In the current study, we further investigated the relation between weight regain and changes in the expression of stress-related genes and stress protein levels to determine possible predictors of weight regain.

Participants/Methods: In this randomized controlled trial, sixty-one healthy overweight/obese participants followed a DI of either a 5-week very-low-calorie diet $(500 \mathrm{kcal} / \mathrm{d})$ or a 12-week lowcalorie diet (1250 kcal/d) (WL period) with subsequent a 4-week weight stable diet (WS period), and a 9-month follow-up. The WL and WS period taken together was named the DI. Abdominal subcutaneous adipose tissue biopsies were collected in fifty-three participants for microarray and liquid chromatography-mass spectrometry analysis. RNA and protein levels for a broad set of stressrelated genes were correlated to the weight regain percentage.

Results: Different gene sets correlated to weight regain percentage during WS and DI. Bioinformatics clustering suggests that during the WS phase defined genes for actin filament dynamics, glucose handling and nutrient sensing are related to weight regain. HIF-1 is indicated as an important regulator. With regard to $\mathrm{DI}$, clustering of correlated genes indicate that LGALS1, ENO1 and ATF2 are important nodes for conferring risk for weight regain.

Conclusions: Our present findings indicate that the risk for weight regain is related to expression changes of distinct sets of stress-related genes during the first four weeks after returning to energy balance, and during the DI. Further research is required to investigate the mechanistic significance of these findings and find targets for preventing weight regain.
\end{abstract}




\section{Introduction}

Overweight and obesity are serious threats to the human health due to an increased risk for the metabolic syndrome and development of type 2 diabetes, cardiovascular diseases and cancer $(1,2)$. There is a simple remedy to obesity i.e. losing weight by dietary intervention, increased physical activity, pharmacological and/or surgical treatment (3). However, long-term weight loss maintenance has been proven to be difficult. In general up to $80 \%$ of the people are unsuccessful in maintaining weight loss $(4,5)$ defined as "keeping off an intentional loss of at least $10 \%$ body weight for at least one year" (6). Therefore, it is of great importance to understand the mechanisms that influence the risk for weight regain. Various factors have already been associated with weight regain or weight maintenance after weight loss such as insulin sensitivity $(7,8)$, fasting insulin and HOMA-IR (9), metabolic factors $(10,11)$, sex hormones $(10)$, hunger and satiety hormones (12) and epigenetic modifications like methylation of the NPY and POMC gene promoters (13). The adipose tissue plays an important role in the increased risk for weight regain. During a negative energy balance, loss of triglycerides decreases the volume of an adipocyte creating stress between the cell contents and the surrounding extracellular matrix (ECM) (14). This stress can be resolved by remodeling of the ECM. However, under a negative energy balance this may be hard or even impossible. Thus, the stress needs to be resolved by another mechanism such as the re-storing of triglycerides within the adipocytes (15). This way the contents of the adipocyte increase to fit the surrounding ECM. It suggests that cellular stress accumulated in adipocytes during a negative energy balance is a driving force behind the risk for weight regain.

Increased stress, especially endoplasmic reticulum (ER) stress, is observed in the subcutaneous adipose tissue of obese people compared to lean people (16). Sharma et al. reported a positive correlation between BMI and ATF67-induced ER stress markers (17). Although these findings suggest that cellular stress increases as a consequence of increasing weight, it might well be that cellular stress is also a factor that stimulates accumulation of fat and is a risk factor for weight regain after weight loss. In a previous study, healthy overweight and obese participants underwent an 8-week calorie-restricted diet with a 10-month follow-up. After weight loss, we observed significantly higher cellular stress in the subcutaneous adipose tissue of participants regaining their lost weight during the follow-up period compared to the participants maintaining weight loss (18). In the present study, our main objective was to further investigate the possible relation between weight regain during follow-up and changes in the expression of genes for stress proteins during weight loss and weight stabilization. This included changes in both the RNA and protein levels for a broad set of stressrelated genes.

\section{Methods}

\section{Participants and study design}

Sixty-one overweight and obese (BMI 27-36 kg/ $\mathrm{m}^{2}$ ) Caucasian participants underwent a dietary intervention (Figure 1). The whole study design has been described in detail previously (19). In short, participants were randomly assigned to either a very-low-calorie diet (VLCD, rapid weight loss) or a low-calorie diet (LCD, slow weight loss) group. Participants in the VLCD group underwent a 5-week diet with about $500 \mathrm{kcal} /$ day by consuming three meal replacements per day (Modifast; Nutrition et Santé Benelux). Participants in the LCD group underwent a 12 -week diet with about $1250 \mathrm{kcal} / \mathrm{day}$ designed by a dietician. Both groups were targeted to lose approximately $10 \%$ body weight during 
this weight loss period (WL, T1-T2). Following weight loss, all participants underwent a 4-week weight maintenance diet based on their individual energy requirements. This weight stable period (WS, T2-T3) was designed to investigate the effect of weight loss of approximately $10 \%$, without the interfering effect of a pronounced negative energy balance. The WL and WS period taken together was named the dietary intervention (DI, T1-T3). The study dietician provided dietary advice according to the Dutch national guidelines (20) to the VLCD and LCD group, to assist in weight loss during the WL period and to assist in remaining weight stable throughout the WS period. After the WS period, participant's body weight was monitored for 9 months (follow-up, T3-T4) by monthly meetings with a dietician. During this follow-up, participants did not receive advice on monitoring and limiting food intake to mimic non-restricted free-living conditions.

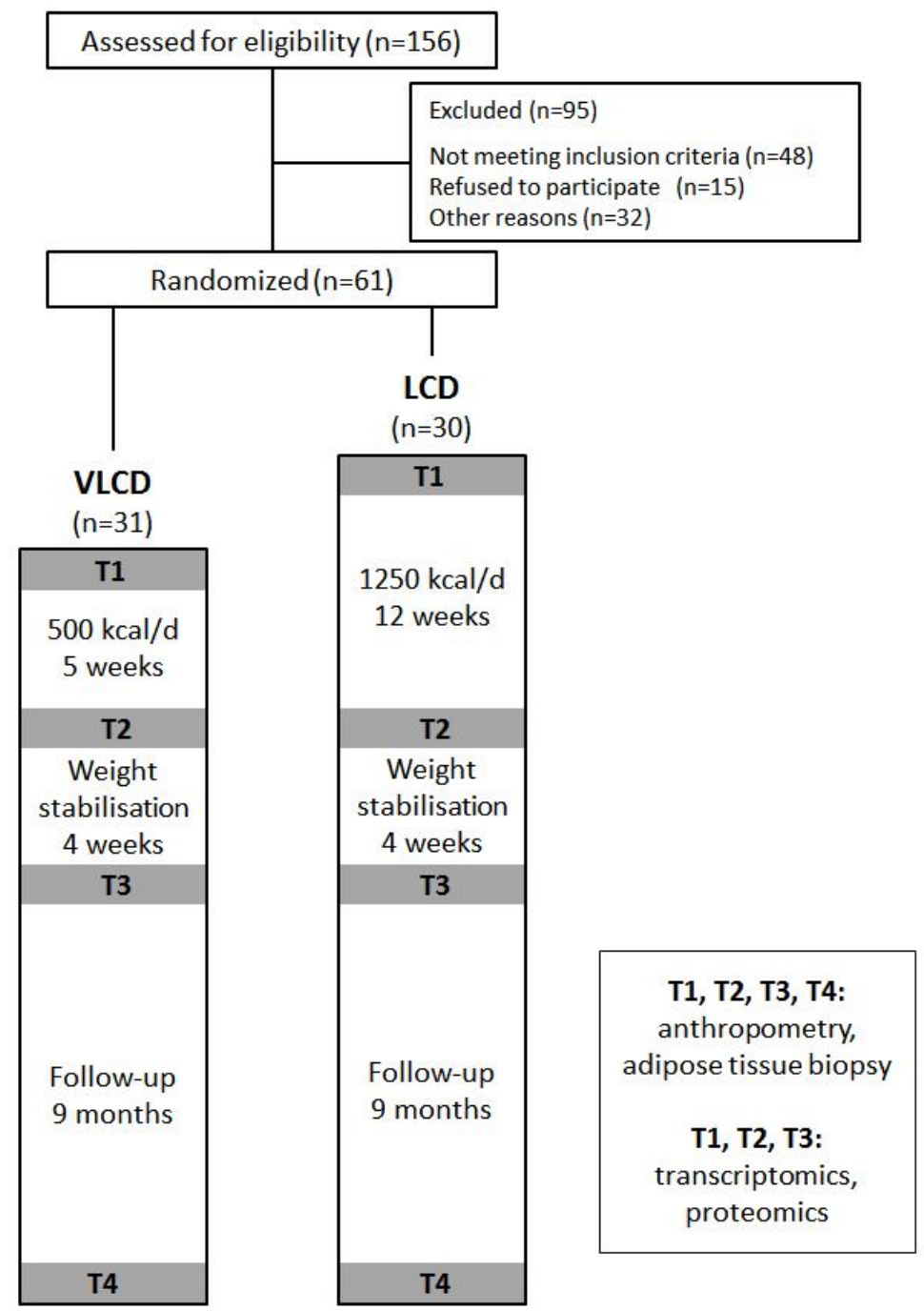

Figure 1: Schematic overview of the study design. Anthropometry measurements and adipose tissue biopsies were obtained at the start of the study (T1), at the end of the weight loss period (T2), weight stable period (T3), and follow-up (T4). Transcriptomics and proteomics were performed at T1, T2 and T3. The dietary intervention period (DI) is the weight loss period and weight stable period taken together.

Before weight loss (T1), after weight loss (T2), after the WS period (T3) and after follow-up (T4) body composition, body weight, height, and waist and hip circumference were measured after overnight fasting (19). Body volume was determined with air-displacement plethysmography using a Bod Pod 
device (Cosmed, Italy, Rome) according to the manufacturer's instructions and as described by Dempster et al. (21). Body composition was calculated from body density according to the twocompartment model by Siri (22).

Four participants withdrew from the study during the dietary intervention (T1-T3), for three participants we could not collect enough biopsy material and for one participant the gene expression results deviated strongly from the others and were therefore excluded. Characteristics of the remaining fifty-three participants at $\mathrm{T} 1, \mathrm{~T} 2$ and $\mathrm{T} 3$ are displayed in Supplement Table 1.

This study was conducted according to the Declaration of Helsinki guidelines and registered on ClinicalTrials.gov (registration number: NCT01559415). All procedures involving human participants were approved by the Central Committee on Human Research and by the Medical Ethical Committee of Maastricht University, The Netherlands. Written informed consent was obtained from all participants.

\section{Adipose tissue biopsy}

Abdominal subcutaneous adipose tissue biopsies were obtained by needle biopsy under local anaesthesia (2\% lidocaine, Fresenius Kabi) after an overnight fast at T1, T2 T3, and T4. Tissue was immediately rinsed in sterile saline, frozen in liquid nitrogen and stored at $-80^{\circ} \mathrm{C}$ until RNA and protein isolation.

\section{Adipose tissue RNA isolation and microarray analysis}

For RNA isolation, adipose tissue samples from T1, T2 and T3 were used. Total RNA was extracted from approx. $150 \mathrm{mg}$ frozen adipose tissue using TRIzol reagent (Invitrogen). Total RNA (100 ng per sample) was labeled by Whole-Transcript Sense Target Assay and hybridized to human wholegenome Affymetrix Gene 1.1 ST arrays targeting 19654 unique genes (Affymetrix). Sample labeling, hybridization to chips and image scanning was performed according to manufacturer's instructions. Microarray signals were normalized using robust multichip average (RMA). Genes with normalized signals $>20$ on at least 15 arrays were defined as expressed (11532 genes). Gene expression changes within groups during a certain period (WL, WS or DI) were defined as significantly different when the q-value was $<0.05$ in a paired t-test with Bayesian correction (Limma) (23). Array data have been submitted to the Gene Expression Omnibus (number GSE77962).

In this study, 107 genes related to stress were selected for the analysis (Supplement Table 2). Sixteen genes were specifically related to endoplasmic reticulum stress as previously reported $(16,17)$, twenty-four candidate stress-related genes were selected based on the frequently detected proteins in subcutaneous adipose tissue (24) and sixty-seven were genes with the term "response to stress" in the Gene Ontology Biological Process (GO-BP) description.

\section{Adipose tissue protein isolation and preparation for liquid chromatography-mass spectrometry (LC-MS/MS)}

For protein isolation, adipose tissue samples from T1, T2 and T3 were used. About $100 \mathrm{mg}$ of the frozen adipose tissue was ground in a mortar with liquid nitrogen. Per mg of grounded powder $2 \mu \mathrm{l}$ of $50 \mathrm{mM}$ ammonium bicarbonate $(A B C)$ with $5 \mathrm{M}$ urea was used to dissolve the powder. Same procedure was done for a control adipose tissue sample, the control subcutaneous adipose tissue 
sample was obtained from a healthy obese during abdominoplasty. The sample was vortexed for 5 minutes. The homogenate was centrifuged for 30 minutes $14000 \mathrm{rpm}$ at $10^{\circ} \mathrm{C}$. The supernatant was carefully collected and protein concentrations were determined with a Bradfort-based protein assay (Bio-Rad). Samples and control were labelled with TMT isobaric mass tagging labelling reagent (10plex, Thermo Scientific) according to manufacturer's protocol. In short, $50 \mu \mathrm{g}$ of protein for each sample was used. The TMT labelling reagents were dissolved in $41 \mu \mathrm{L}$ acetonitrile per vial. The reduced and alkylated samples and control were added to the TMT Reagent vial. The reaction was incubated for 1 hour at room temperature and quenched for 15 minutes by adding $8 \mu \mathrm{L}$ of $5 \%$ hydroxylamine. The samples and control were combined in a new microcentrifuge tube at equal amounts and analysed by LC-MS/MS.

\section{Liquid chromatography-mass spectrometry analysis for protein quantification}

A nanoflow HPLC instrument (Dionex ultimate 3000) was coupled on-line to a Q Exactive (Thermo Scientific) with a nano-electrospray Flex ion source (Proxeon). The final concentration of the TMT labeled digest/peptide mixture was $0.2 \mu \mathrm{g} / \mu \mathrm{l}$ and $5 \mu \mathrm{l}$ of this mixture was loaded onto a C18reversed phase column (Thermo Scientific, Acclaim PepMap C18 column, 75- $\mu$ m inner diameter x 15 $\mathrm{cm}, 5-\mu \mathrm{m}$ particle size). The peptides were separated with a 120 minutes linear gradient of $4-45 \%$ buffer B ( $80 \%$ acetonitrile and $0.08 \%$ formic acid) at a flow rate of $300 \mathrm{~nL} / \mathrm{min}$.

MS data was acquired using a data-dependent top10 method, dynamically choosing the most abundant precursor ions from the survey scan $(280-1500 \mathrm{~m} / \mathrm{z})$ in positive mode. Survey scans were acquired at a resolution of 70,000 and a maximum injection time of $120 \mathrm{~ms}$. Dynamic exclusion duration was $30 \mathrm{~s}$. Isolation of precursors was performed with a $2.0 \mathrm{~m} / \mathrm{z}$ window. Resolution for HCD spectra was set to 35,000 and the normalized collision energy was $30 \mathrm{eV}$. The under fill ratio was defined as $1.0 \%$. The instrument was run with peptide recognition mode enabled, but exclusion of singly charged and charge states of more than five.

\section{Database search}

The MS data were searched using Sequest HT Proteome Discoverer 2.1 search engine (Thermo Scientific) against the UniProt human database. The false discovery rate (FDR) was set to 0.01 for proteins and peptides, which had to have a minimum length of 6 amino acids. The precursor mass tolerance was set at $10 \mathrm{ppm}$ and the fragment tolerance at $0.2 \mathrm{Da}$. One miss-cleavage was tolerated, oxidation of methionine was set as a dynamic modification and carbamidomethylation of cysteines, TMT reagent adducts $(+229.162932 \mathrm{Da})$ on lysine and peptide amino termini were set as fixed modifications.

In total, twenty-three TMT runs were performed. We corrected our data for possible differences between runs and within each run. We corrected for between run differences as follows: (average protein abundance of the twenty-three controls $\div$ protein abundance of control in same run as the test sample) $\mathrm{x}$ protein abundance in the test sample. For the correction within the run, we chose 167 proteins (excluding obvious plasma proteins) which were present in each test sample and used those 167 proteins to calculate the average protein abundance of each test sample. We corrected for within run differences as follows: (average total protein abundance of the control $\div$ total protein abundance of the test sample) $x$ original protein abundance in the test sample. 


\section{Calculations}

In this study we wanted to analyse the relation between gene expression and protein level changes and weight regain and/or maintenance. The objective was to determine possible predictors of weight regain during WL, WS or DI. As a value for weight regain and/or maintenance, we calculated the weight change percentage during follow-up as follows: ((weight after follow-up T4 - weight after WS T3) $\div$ weight after WS T3) $\times 100 \%$. For gene expression change we calculated the fold change (FC) of gene expression during WL, WS or DI. Gene expression results from the microarray were expressed as $\log _{2}$ transformed values. Gene expression changes during a certain period were calculated as follows: WL ( $\left.\log _{2} T 2-\log _{2} T 1\right)$, WS $\left(\log _{2} T 3-\log _{2}\right.$ T2) and DI $\left(\log _{2} T 3-\log _{2} T 1\right)$. These $\log _{2}$ ratios (LR) during $W L$, WS and $D I$ were transformed into fold changes $\left(F C=2^{L R}\right.$ if $L R \geq 0$ and $F C=(-1) 2^{-L R}$ if $\left.L R<0\right)$.

\section{Bioinformatics analyses}

Gene clustering was done by the Human Consensus Path DataBase (CPDB release 31, http://cpdb.molgen.mpg.de/). From 'gene set analysis' the 'induced network modules' tool was chosen and the list of genes significantly correlated with the weight regain percentage during WS or DI was entered (so-called seed genes). The program aims to interconnect the seed genes through different types of interactions (such as biochemical, regulatory, genetic or protein interactions). The induced network modules may include genes that are not in the user-supplied seed list, but associate two or more seed genes with each other and overall have significantly many connections within the induced network module (so-called intermediate nodes). The gene clustering during WS and DI focussing on biochemical reactions and gene regulatory interactions with allowance for intermediate nodes (z-score: 20). Also, gene clustering focussing on protein interactions was done for the 8 correlating genes of the DI phase with allowance for intermediate nodes (z-score: 20 ).

\section{Statistical analysis}

Dependent T-test was carried out to determine possible effects over time within a group. Comparisons between VLCD and LCD were made with independent T-test. Weight change (\%) during follow-up was correlated with fold change of gene expression and protein levels during WL, WS and DI. Correlations were made using the Pearson correlation coefficient. Statistical analyses were done using SPSS 20.0 for Windows (SPSS Inc). For all statistical tests $\mathrm{P} \leq 0.05$ was considered to be statistically significant, unless otherwise stated. Data are presented as mean \pm SEM. Variation in the number of participants between analyses is due to the exclusion of participants with missing data. All variables were checked for normal distribution, and variables with a skewed distribution were Intransformed to satisfy conditions of normality. Extreme outliers (values higher than $3 x$ interquartile range calculated with SPSS) influencing the data were removed during statistical analyses.

\section{Results}

\section{Changes of subject characteristics throughout the study}

At the start of this study, no significant differences were observed between VLCD and LCD (Supplement Table 1). After WL and WS, all parameters remained similar between VLCD and LCD. During the WL period, body weight, BMI, hip circumference, waist circumference, body fat percentage, body fat and fat free mass were significantly decreased in both the VLCD and the LCD group (Table 1). In the subsequent 4 weeks (WS period), body fat and body fat percentage decreased for both VLCD and LCD, whereas body weight did not change (Supplement Table 1). Also, an increase 
in fat free mass is observed in the VLCD group. In the LCD group, hip circumference significantly increased. During the dietary intervention (T1-T3), all parameters significantly decreased except for fat free mass in the LCD group (Table 1). Body weight, BMI, waist circumference, body fat percentage, body fat and fat free mass increased during follow-up (T3-T4). The average weight regain percentage did not differ between VLCD and $\operatorname{LCD}(5.4 \pm 4.5 \%$ and $5.3 \pm 3.8 \%, P=0.957$, Supplement Figure 1).

Table 1: Changes in characteristics at the end of weight loss and the dietary intervention compared to study start, and at the end of follow-up compared to the end of the dietary intervention.

\begin{tabular}{|c|c|c|c|c|c|c|}
\hline & \multicolumn{2}{|c|}{$\begin{array}{l}\text { End of WL vs study start } \\
\text { (T2 vs T1) }\end{array}$} & \multicolumn{2}{|c|}{$\begin{array}{l}\text { End of DI vs study start } \\
\text { ( }(\mathrm{T} 3 \mathrm{vs} \mathrm{T} 1 \text { ) }\end{array}$} & \multicolumn{2}{|c|}{$\begin{array}{l}\text { End of follow-up vs end of DI } \\
\text { (T4 vs T3) }\end{array}$} \\
\hline & $\operatorname{VLCD}(n=26)$ & $\operatorname{LCD}(n=27)$ & $\operatorname{VLCD}(\mathrm{n}=26)$ & $\operatorname{LCD}(n=27)$ & $\operatorname{VLCD}(n=26)$ & $\operatorname{LCD}(n=27)$ \\
\hline Weight (kg) & $-8.9 \pm 0.4^{* * *}$ & $-8.1 \pm 0.5^{* * *}$ & $-9.1 \pm 0.5^{* * *}$ & $-8.3 \pm 0.6^{* * *}$ & $-9.1 \pm 0.5^{* * *}$ & $-8.3 \pm 0.6^{* * *}$ \\
\hline $\mathrm{BMI}\left(\mathrm{kg} / \mathrm{m}^{2}\right)$ & $-3.0 \pm 0.1^{* * *}$ & $-2.8 \pm 0.2 * * *$ & $-3.0 \pm 0.2^{* * *}$ & $-2.8 \pm 0.2^{* * *}$ & $-3.0 \pm 0.2^{* * *}$ & $-2.8 \pm 0.2^{* * *}$ \\
\hline Hip circumference $(\mathrm{cm})$ & $-6.0 \pm 0.7^{* * *}$ & $-4.5 \pm 0.6 * * *$ & $-6.1 \pm 0.7^{* * *}$ & $-5.9 \pm 0.7^{* * *}$ & $-6.1 \pm 0.7^{* * *}$ & $-5.9 \pm 0.7 * * *$ \\
\hline Waist circumference $(\mathrm{cm})$ & $-7.8 \pm 0.7^{* * *}$ & $-7.3 \pm 0.8 * * *$ & $-6.7 \pm 0.7^{* * *}$ & $-8.0 \pm 1.0 * * *$ & $-6.7 \pm 0.7^{* * *}$ & $-8.0 \pm 1.0^{* * *}$ \\
\hline Body fat (\%) & $-4.7 \pm 0.4^{* * *}$ & $-5.4 \pm 0.6 * * *$ & $-5.9 \pm 0.5^{* * *}$ & $-6.0 \pm 0.5^{* * *}$ & $-5.9 \pm 0.5^{* * *}$ & $-6.0 \pm 0.5^{* * *}$ \\
\hline Body fat (kg) & $-7.3 \pm 0.4^{* * *}$ & $-7.6 \pm 0.6 * * *$ & $-8.4 \pm 0.5^{* * *}$ & $-8.1 \pm 0.6^{* * *}$ & $-8.4 \pm 0.5^{* * *}$ & $-8.1 \pm 0.6^{* * *}$ \\
\hline Fat free mass $(\mathrm{kg})$ & $-1.6 \pm 0.2^{* * *}$ & $-0.5 \pm 0.2 *$ & $-0.8 \pm 0.2^{* *}$ & $-0.2 \pm 0.2$ & $-0.8 \pm 0.2^{* *}$ & $-0.2 \pm 0.2$ \\
\hline
\end{tabular}

\section{Changes of stress gene RNA and protein levels correlate with weight regain}

Changes during WL, WS and DI of the expression of 17 out of the 107 stress-related genes correlated with weight regain percentage (Table 2). Most correlations were found in the VLCD group (19) as compared to the LCD group (2), which may be due to the fact that gene expression changes after the VLCD are considerably more profound than after the LCD (25) allowing significant correlations to be more readily detected. All correlations observed have a $|r| \geq 0.5$ indicating that these gene changes are correlating strongly with the weight regain percentage. Aldolase $\mathrm{A}(A L D O A)$ is the only gene correlating with weight regain in both VLCD and LCD. However, the observed correlations were in different phases, WS for the VLCD group $(r=0.552, P=0.006)$ and $W L$ for the LCD group $(r=0.590$, $\mathrm{P}=0.003)$. Remarkably, in the VLCD group hardly any correlations were found for the WL period, but mainly for the WS and DI phases. In the VLCD group, during the WS period the genes most strongly correlating with weight regain percentage are Enolase 1 (ENO1, $r=0.710, \mathrm{P}<0.001$ ), Protein tyrosine kinase 2 beta (PTK2B, $r=0.658, \mathrm{P}=0.001$ ) and Phosphoglycerate kinase 1 ( $P G K 1, r=0.655, \mathrm{P}=0.001$ ), and during DI Lectin, galactoside-binding, soluble, 1 (LGALS1, $r=0.705, P<0.001), E N O 1$ ( $r=0.684$, $\mathrm{P}<0.001)$ and Crystallin, alpha $\mathrm{B}(C R Y A B, r=0.671, \mathrm{P}<0.001)$. 
Table 2: Correlation coefficients between the weight regain percentage and the fold changes in gene expression levels during the weight loss, weight stable and dietary intervention period.

\begin{tabular}{|c|c|c|c|c|c|c|c|}
\hline \multirow[t]{2}{*}{ Gene ID } & \multirow[t]{2}{*}{ Gene name } & \multicolumn{3}{|l|}{$\overline{V L C D}$} & \multicolumn{3}{|l|}{ LCD } \\
\hline & & WL & WS & DI & WL & WS & DI \\
\hline ACTB & Actin, beta & & $0.562^{*}$ & & & & \\
\hline ALDOA & Aldolase A & & 0.552 & & $0.590^{*}$ & & \\
\hline ANXA5 & Annexin A5 & & & $0.566^{*}$ & & & \\
\hline C5 & Complement component 5 & & -0.525 & & & & \\
\hline CFL1 & Cofilin 1 & & $0.607^{*}$ & & & & \\
\hline CRHBP & $\begin{array}{l}\text { Corticotropin releasing hormone } \\
\text { binding protein }\end{array}$ & & & -0.518 & & & \\
\hline CRYAB & Crystallin, alpha B & $0.586^{*}$ & & $0.671^{*}$ & & & \\
\hline ENO1 & Enolase 1 & & $0.710^{*}$ & $0.684^{*}$ & & & \\
\hline GSTP1 & Glutathione S-transferase pi 1 & & & $0.597^{*}$ & & & \\
\hline LGALS1 & Lectin, galactoside-binding, soluble, 1 & & & $0.705^{*}$ & & & \\
\hline MAPK13 & Mitogen-activated protein kinase 13 & & $0.586^{*}$ & & & & \\
\hline PGK1 & Phosphoglycerate kinase 1 & & $0.655^{*}$ & & & & \\
\hline PHB & Prohibitin & & & 0.528 & & & \\
\hline PRDX6 & Peroxiredoxin 6 & & & & & $0.606 *$ & \\
\hline PTK2B & Protein tyrosine kinase 2 beta & -0.547 & $0.658^{*}$ & & & & \\
\hline RPS6KA1 & $\begin{array}{l}\text { Ribosomal protein } \mathrm{S} 6 \text { kinase, } 90 \mathrm{kDa} \text {, } \\
\text { polypeptide } 1\end{array}$ & & $0.643^{*}$ & & & & \\
\hline UCN2 & Urocortin 2 & & & -0.521 & & & \\
\hline
\end{tabular}

In order to see if correlations could be found between changes in protein level and weight regain percentage, we performed quantitative analysis by LC-MS/MS of adipose tissue proteins for the same three time points. In total, we were able to measure 22 proteins of the 107 stress-related genes (Supplement Table 3). Nine proteins correlated with weight regain percentage. The strongest correlation observed was with Heat shock protein beta-6 (HSPB6, $r=0.800, \mathrm{P}=0.003$ ) during the WS period (Table 3). In the VLCD group, two proteins were correlated with weight regain which were also correlated on gene level: $\beta$-Actin and Phosphoglycerate kinase 1, although during different phases ( $\beta$ Actin: WL; PGK1: DI).

Table 3: Correlation coefficients between the weight regain percentage and the fold changes in protein levels during the weight loss, weight stable and dietary intervention period.

\begin{tabular}{|c|c|c|c|c|c|c|c|c|}
\hline \multirow[t]{2}{*}{ Protein name } & \multirow[t]{2}{*}{ Uniprot ID } & \multirow[t]{2}{*}{ Gene ID } & \multicolumn{3}{|l|}{ VLCD } & \multicolumn{3}{|l|}{ LCD } \\
\hline & & & WL & WS & DI & WL & WS & DI \\
\hline $60 \mathrm{kDa}$ heat shock protein & P10809 & HSPD1 & & & & & 0.489 & \\
\hline $78 \mathrm{kDa}$ glucose-regulated protein & P11021 & GRP78 & & & & 0.499 & & \\
\hline Actin, cytoplasmic 1 & P60709 & АСТВ & -0.502 & & & & & \\
\hline Galectin-1 & P09382 & LGALS1 & & & & -0.421 & & \\
\hline Heat shock protein beta- 6 & 014558 & HSPB6 & & 0.800 & & & & \\
\hline Nucleophosmin & P06748 & NPM & & & & & & -0.617 \\
\hline Phosphoglycerate kinase 1 & P00558 & PGK1 & & & 0.589 & & & \\
\hline Pyruvate kinase & P14618 & PKM & -0.526 & 0.564 & & & & \\
\hline Triosephosphate isomerase & P60174 & TPI1 & & 0.510 & & & & \\
\hline
\end{tabular}




\section{Bioinformatics analysis of the correlated genes}

Only one of the correlated genes (ENO1) shows up in both the VLCD WS and DI phases (Table 2). This poor overlap suggests that these genes represent two different functional or regulatory gene sets. Clustering analyses based on biochemical reactions and gene regulatory interactions showed that 8 of 9 correlating genes of the WS phase were clustered (Figure 2A), whereas clustering in this way was not apparent for correlating genes of the DI phase. Moreover, combined analysis of all genes did not change the observed cluster except for interactions of ANXA5 and GSTP1 with the intermediate node ISG15. It confirms that both gene sets are distinct. The HIF-1 complex was revealed as an important regulator. Interestingly, the gene $C 5$ that was not made part of the cluster by the bioinformatics analysis, has two binding sites for HIF-1A at 66 and 1556 base pairs before the start site of transcription as shown in the GeneCards human gene database (www.genecards.org). Although clustering as for the WS correlating genes gave no result with correlating genes of the DI phase, 7 of 8 correlating genes of the DI phase could be clustered with the same tool but focusing on protein interactions (Figure 2B). Besides ATF2, most connections were with LGALS1 and ENO1, which correspond to the genes with the strongest correlations with weight regain percentage. However, all connections were through intermediate nodes.

A

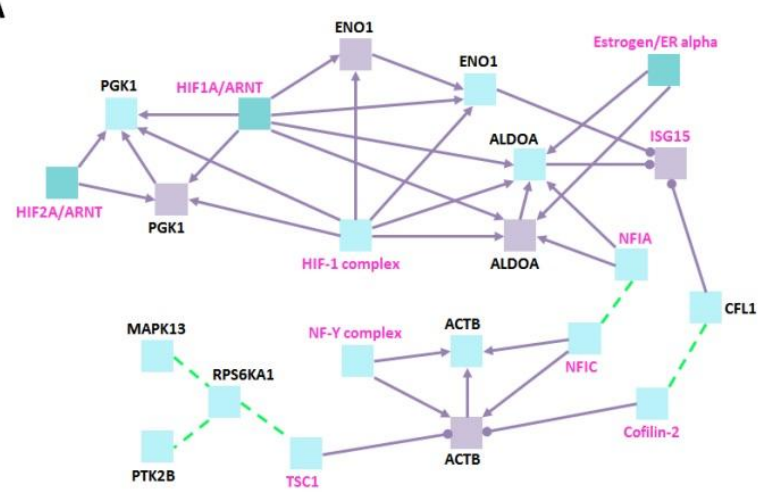

B

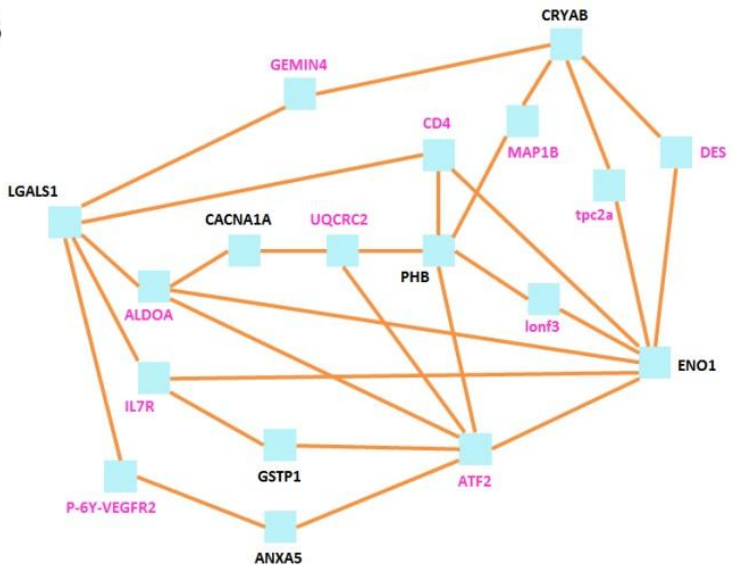

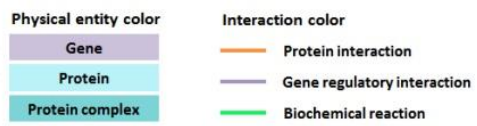

Figure 2: Interactions of genes correlated with weight regain in the VLCD group during WS or DI. Gene clustering during the WS phase focussed on biochemical reactions and gene regulatory interactions with allowance for intermediate nodes ( $z$ score: 20) (A) and gene clustering during the DI phase focussed on protein interactions with allowance for intermediate nodes (z-score: 20) (B). Black gene names: significantly correlated with weight regain (so-called seed genes) and pink gene names: genes not in the user-supplied seeds list (so-called intermediate genes).

\section{Discussion}

In the present study we investigated the relation between weight regain percentage and changes in expression of stress-related genes, both on the RNA and protein level, during weight loss by VLCD or LCD and during a weight stabilisation period. We found that correlations between changes in RNA levels and weight regain were more frequent for the VLCD group than for the LCD group. Unexpectedly, correlations were spurious for the WL phase, almost all appeared in the WS or DI phases. Remarkably, the correlating genes of the WS phase were different from the genes correlating in the DI phase. 
More and stronger correlations were found in the VLCD compared to the LCD group. The total amount of weight loss was similar in both groups, however, on a VLCD the negative energy balance is more profound and induces stronger responses in gene expression, which probably makes the detection of significant correlations easier. A stronger VLCD response was not only seen in stressrelated genes but appeared to be the case for most other genes as well(25). On the other hand, decreasing the threshold for statistical significance from $\mathrm{P} \leq 0.01$ to $\mathrm{P} \leq 0.05$ reduces the difference between VLCD and LCD in number of detected correlations (Supplement table 4). Thus, the difference is probably a consequence of both the negative energy balance and the applied threshold. For the protein analysis we used $\mathrm{P} \leq 0.05$ as cut-off for significance, which may explain that there was no obvious difference in number of correlations between VLCD and LCD.

Surprisingly, correlations between changes in RNA levels and weight regain percentage were mainly found during the WS and DI phases and not in the WL phase. In other studies differences have been observed in subcutaneous adipose tissue gene expression patterns after WL between participants maintaining or regaining their weight after weight loss (26-28). However, in these studies participants were split into a weight maintaining and a weight regaining group to show that some genes were differentially expressed between these groups. Still other studies focussed more on genetic variation associated with weight regain $(29,30)$. The present study is one of the few that included a weight stable period to look at gene expression changes in relation to weight regain. During the WL phase there is a strong negative energy balance which may lead to low levels of cellular energy especially in the VLCD group. These conditions may outbalance the expected responses of stress-related genes disturbing the detection of significant relations. During WS and DI, when sufficient cellular energy is available, correlations may be more readily detected.

Bioinformatics analysis revealed that 8 of 9 correlating genes of the WS phase were clustered. The functions of these genes can roughly be divided into 3 processes: cytoskeleton organisation, glycolysis and nutrient sensing. Four genes have a link with the cytoskeleton: ACTB, CFL1, ALDOA and PTK2B. $\beta$-Actin is a structural component of actin filaments, which are located below the cell membrane. Cofilin-1 can bind to F-actin and is able to depolymerise the filaments allowing dynamic changes of the actin cytoskeleton (31). Binding of cofilin to actin can lead to the accumulation of lipids in the adipose tissue, because cofilin controls the mechanical tension of the adipocyte $(32,33)$. Aldolase has previously been recognized as a component of the cytoskeleton, binding to the actin filaments, where it may serve in anchoring and translocation of the GLUT4-containing vesicles (34, 35). Based on proteomics analysis in a weight loss-maintenance study, we have previously proposed a mechanism in which phosphorylation of Annexin 2 at Tyr-24 mediates the translocation of GLUT4 vesicles from the Aldolase-anchored position at the actin filaments to the cell membrane, resulting in increased uptake of glucose (36). In 3T3-L1 cells it has been shown that upon stimulation PYK2, the murine ortholog of PTK2B, induces the formation of actin filaments and mediates independent of insulin the endothelin-1stimulated translocation of GLUT4 to the membrane (37). As such, our present findings are completely in line with an upregulation of glucose uptake shortly after returning to an energy balanced state. Moreover, we now find that this potential upregulation is related to the risk for weight regain. It is substantiated by the fact that besides Aldolase A, two other genes coding for enzymes of the glycolysis, PGK1 and ENO1, are strongly correlated with weight regain percentage. In addition, on the protein level another two glycolytic enzymes, PKM and TPI1, correlated with weight regain percentage during the WS phase in the VLCD group. 
The bioinformatics analysis indicates that the HIF-1 complex may have an important regulatory role in determining the expression of the glycolytic genes. In the VLCD group HIF-1A gene expression dramatically increases during $W L(F C=1.25, Q<0.0001)$ and decreases during $W S(F C=-1.14, Q=0.02)$. Expression of the genes for ALDOA, ENO1 and PGK1 significantly decrease during WL, but do not change during WS suggesting that HIF-1 is not the only regulatory factor. Also the correlated glycolytic proteins PKM and TPI are known to be regulated by HIF-1 (38). Notably, the expression of the $C 5$ gene parallels that of HIF-1A during WL ( $F C=1.27, Q<0.0001)$ and $W S(F C=-1.15, Q=0.01)$. The meaning of the inverse correlation of $C 5$ gene expression during WS with weight regain percentage is unclear. However, a role for $\mathrm{C} 5$ in obese adipose tissue inflammation and insulin resistance has been proposed (39). Unfortunately, the role of the HiF1 protein could not be investigated, because it was not detected by LC-MSMS and no sample was left to perform a different way of protein quantification.

It can be expected that the influx of glucose and the availability of metabolic energy triggers nutrientsensing pathways. An important player is the mTOR Complex 1 , which is inhibited by the TSC2-TSC1 complex. RPS6KA1 is able to phosphorylate TSC2 at Ser1798, which leads to activation of the mTORC1 signalling $(40,41)$. In addition, MAPK13 is a regulator of the amino acid-induced activation of the mTOR signalling (42). Using rat ELT3 cells it was shown that TSC1 functions in actin dynamics, cell adhesion and stress fiber (dis)assembly (43). As indicated by the position of TSC1 in the cluster, the TSC complex may link nutrient sensing to modification of the actin cytoskeleton.

Analysis of the genes correlating with weight regain percentage during DI led to a protein interaction cluster with ENO1, LGALS1 and ATF2 as most involved nodes. ATF2 belongs to the ATF/CREB transcription factor family. Its activation is effectuated by stress-activated protein kinases such as MAPK13/p38ס. In the mouse, inhibition of p38 reduced high-fat diet induced obesity and insulin resistance (44). The clustering results suggest that its transactivating activity may also be influenced by interaction with proteins encoded by the correlated genes and as such modify the risk of weight regain. Another nodal protein is galectin-1. Several pharmacological inhibitors of this protein have shown to lower body weight gain in diet-induced obese rats suggesting that this protein can also be a target for preventing weight regain (45-47).

Our present results show that changes in expression of defined stress-related genes correlate to the risk for weight regain in the WS and DI phase. In a previous weight loss study we reported that higher levels of the stress proteins $\beta$-actin, HSP27, HSP60 and HSP70 in adipose tissue were associated with regaining weight after weight loss on a VLCD. Here, we have not compared gene- and protein expression between weight regainers and weight maintainers, but searched for correlations between gene/protein expression and weight regain percentage. Indications for involvement of those genes in weight regain of the present VLCD group were mainly found in the gene expression data of the WS phase: B-actin ( $r=0.562, \mathrm{P}=0.003)$, HSP27 ( $r=0.470, \mathrm{P}=0.02)$, HSP70/HSPA5 $(r=0.457, \mathrm{P}=0.03)$ and HSP70/HSPA8 ( $r=0.535, \mathrm{P}=0.007)$. On the protein level, HSP60/HSPD1 correlated with weight regain during the WS period but only in the LCD group $(r=0.489, \mathrm{P}=0.03)$. All correlations are positive indicating that an increase of stress or a lower decrease of stress is in line with risk for weight regain. A limitation of the current study is that we were not able to measure all stress-related proteins because the abundance of some proteins including HIF-1 could not be properly determined by LCMS/MS. Yet, we were still able to measure twenty-two different stress proteins. Furthermore, a 
relatively high number of participants were included in the microarray analysis which increases the strength of the transcriptomics data. For correlations with gene expression data we used a cut-off Pvalue of 0.01 and several correlations were even $<0.001$. Clustering suggested coherence of correlating gene sets. Nevertheless, false positives cannot be excluded. This is especially the case for the protein correlations for which a threshold $\mathrm{P}$-value of 0.05 was used.

In conclusion, our present findings indicate that the risk for weight regain is related to changes in the expression of stress-related genes during the first four weeks after returning to a weight stable situation (WS), and during the whole dietary intervention (DI, WS+WL). Bioinformatics clustering suggests that during the weight stabilisation phase defined genes for actin filament dynamics, glucose handling and nutrient sensing are related to weight regain. HIF-1 is indicated as an important regulator. With regard to DI, clustering of correlated genes indicate that LGALS1, ENO1 and ATF2 are important nodes for conferring risk to weight regain. Further research is required to investigate the mechanistic significance of the present findings and to try and find targets for prevention of weight regain. 


\section{References}

1. Bessesen DH. Update on obesity. J Clin Endocrinol Metab, 2008. 93(6):2027-34.

2. Wing RR, Hill JO. Successful weight loss maintenance. Annu Rev Nutr, 2001. 21:323-41.

3. Wu T, Gao X, Chen M, van Dam RM. Long-term effectiveness of diet-plus-exercise interventions vs. diet-only interventions for weight loss: a meta-analysis. Obes Rev, 2009. 10(3):313-23.

4. Barte JC, ter Bogt NC, Bogers RP, Teixeira PJ, Blissmer B, Mori TA, Bemelmans WJ. Maintenance of weight loss after lifestyle interventions for overweight and obesity, a systematic review. Obes Rev, 2010. 11(12):899-906.

5. Elfhag $\mathrm{K}$, Rossner $\mathrm{S}$. Who succeeds in maintaining weight loss? A conceptual review of factors associated with weight loss maintenance and weight regain. Obes Rev, 2005. 6(1):67-85.

6. Yost TJ, Jensen DR, Eckel RH. Weight regain following sustained weight reduction is predicted by relative insulin sensitivity. Obes Res, 1995. 3(6):583-7.

7. Wing RR. Insulin sensitivity as a predictor of weight regain. Obes Res, 1997. 5(1):24-9.

8. Wong $\mathrm{MH}$, Holst $\mathrm{C}$, Astrup A, Handjieva-Darlenska T, Jebb SA, Kafatos A et al. Caloric restriction induces changes in insulin and body weight measurements that are inversely associated with subsequent weight regain. PLoS One, 2012. 7(8):e42858.

9. Wang P, Menheere PP, Astrup A, Andersen MR, van Baak MA, Larsen TM et al. Metabolic syndrome, circulating RBP4, testosterone, and SHBG predict weight regain at 6 months after weight loss in men. Obesity (Silver Spring), 2013. 21(10):1997-2006.

10. Aller EE, Larsen TM, Claus $H$, Lindroos AK, Kafatos A, Pfeiffer A et al. Weight loss maintenance in overweight subjects on ad libitum diets with high or low protein content and glycemic index: the DIOGENES trial 12-month results. Int J Obes (Lond), 2014. 38(12):1511-7.

11. Crujeiras AB, Pardo M, Arturo RR, Navas-Carretero S, Zulet MA, Martinez JA, Casanueva FF. Longitudinal variation of circulating irisin after an energy restriction-induced weight loss and following weight regain in obese men and women. Am J Hum Biol, 2014. 26(2):198-207.

12. Wang $\mathrm{P}$, Holst C, Wodzig WK, Andersen MR, Astrup A, van Baak MA et al. Circulating ACE is a predictor of weight loss maintenance not only in overweight and obese women, but also in men. Int $J$ Obes (Lond), 2012. 36(12):1545-51.

13. Crujeiras AB, Goyenechea E, Abete I, Lage M, Carreira MC, Martinez JA, Casanueva FF. Weight regain after a diet-induced loss is predicted by higher baseline leptin and lower ghrelin plasma levels. $J$ Clin Endocrinol Metab, 2010. 95(11):5037-44.

14. Crujeiras AB, Campion J, Diaz-Lagares A, Milagro Fl, Goyenechea E, Abete I, Casanueva FF, Martinez JA. Association of weight regain with specific methylation levels in the NPY and POMC promoters in leukocytes of obese men: a translational study. Regul Pept, 2013. 186:1-6.

15. Vogels N, Diepvens K, Westerterp-Plantenga MS. Predictors of long-term weight maintenance. Obes Res, 2005. 13(12):2162-8.

16. Vogels N, Westerterp-Plantenga MS. Successful long-term weight maintenance: a 2-year follow-up. Obesity (Silver Spring), 2007. 15(5):1258-66.

17. Mauriege $P$, Imbeault $P$, Doucet $E$, Lacaille $M$, Langin D, Almeras N, Despres JP, Tremblay A. Weight loss and regain in obese individuals: a link with adipose tissue metabolism indices? Journal of physiology and biochemistry, 2013. 69(3):497-505.

18. Koppo K, Siklova-Vitkova M, Klimcakova E, Polak J, Marques MA, Berlan M et al. Catecholamine and insulin control of lipolysis in subcutaneous adipose tissue during long-term diet-induced weight loss in obese women. Am J Physiol Endocrinol Metab, 2012. 302(2):E226-32.

19. Verhoef SP, Camps SG, Bouwman FG, Mariman EC, Westerterp KR. Physiological response of adipocytes to weight loss and maintenance. PLoS One, 2013. 8(3):e58011.

20. Boden G, Duan X, Homko C, Molina EJ, Song W, Perez O, Cheung P, Merali S. Increase in endoplasmic reticulum stress-related proteins and genes in adipose tissue of obese, insulin-resistant individuals. Diabetes, 2008. 57(9):2438-44.

21. Sharma NK, Das SK, Mondal AK, Hackney OG, Chu WS, Kern PA, Rasouli N, Spencer HJ, YaoBorengasser A, Elbein SC. Endoplasmic reticulum stress markers are associated with obesity in nondiabetic subjects. J Clin Endocrinol Metab, 2008. 93(11):4532-41.

22. Gregor MF, Yang L, Fabbrini E, Mohammed BS, Eagon JC, Hotamisligil GS, Klein S. Endoplasmic reticulum stress is reduced in tissues of obese subjects after weight loss. Diabetes, 2009. 58(3):693700 . 
23. Krautbauer S, Eisinger K, Hader Y, Neumeier M, Buechler C. Manganese superoxide dismutase knockdown in 3T3-L1 preadipocytes impairs subsequent adipogenesis. Mol Cell Biochem, 2014. 393(1-2):6976.

24. Liu GS, Chan EC, Higuchi M, Dusting GJ, Jiang F. Redox mechanisms in regulation of adipocyte differentiation: beyond a general stress response. Cells, 2012. 1(4):976-93.

25. Shawlot W, Deng JM, Fohn LE, Behringer RR. Restricted beta-galactosidase expression of a hygromycin-lacZ gene targeted to the beta-actin locus and embryonic lethality of beta-actin mutant mice. Transgenic Res, 1998. 7(2):95-103.

26. Kleizen B, Braakman I. Protein folding and quality control in the endoplasmic reticulum. Curr Opin Cell Biol, 2004. 16(4):343-9.

27. Guerin R, Arseneault G, Dumont S, Rokeach LA. Calnexin is involved in apoptosis induced by endoplasmic reticulum stress in the fission yeast. Mol Biol Cell, 2008. 19(10):4404-20.

28. Bousette N, Abbasi C, Chis R, Gramolini AO. Calnexin silencing in mouse neonatal cardiomyocytes induces Ca2+ cycling defects, ER stress, and apoptosis. J Cell Physiol, 2014. 229(3):374-83.

29. Garrido C. Size matters: of the small HSP27 and its large oligomers. Cell Death Differ, 2002. 9(5):483-5.

30. Horman S, Galand P, Mosselmans R, Legros N, Leclercq G, Mairesse N. Changes in the phosphorylation status of the $27 \mathrm{kDa}$ heat shock protein (HSP27) associated with the modulation of growth and/or differentiation in MCF-7 cells. Cell Prolif, 1997. 30(1):21-35.

31. Marker T, Sell H, Zillessen P, Glode A, Kriebel J, Ouwens DM et al. Heat shock protein 60 as a mediator of adipose tissue inflammation and insulin resistance. Diabetes, 2012. 61(3):615-25.

32. Mayer RJ, Ciechanover AJ, Rechsteiner M. Protein degradation. Weinheim: Wiley-VCH, 2005.

33. Toichi K, Yamanaka K, Furukawa Y. Disulfide scrambling describes the oligomer formation of superoxide dismutase (SOD1) proteins in the familial form of amyotrophic lateral sclerosis. $J$ Biol Chem, 2013. 288(7):4970-80.

34. Wang P, Bouwman FG, Mariman EC. Generally detected proteins in comparative proteomics--a matter of cellular stress response? Proteomics, 2009. 9(11):2955-66.

35. Wabitsch M, Brenner RE, Melzner I, Braun M, Moller P, Heinze E, Debatin KM, Hauner $H$. Characterization of a human preadipocyte cell strain with high capacity for adipose differentiation. Int J Obes Relat Metab Disord, 2001. 25(1):8-15.

36. Renes J, Rosenow A, Roumans N, Noben JP, Mariman EC. Calorie restriction-induced changes in the secretome of human adipocytes, comparison with resveratrol-induced secretome effects. Biochim Biophys Acta, 2014.

37. Bouwman FG, Claessens M, van Baak MA, Noben JP, Wang P, Saris WH, Mariman EC. The physiologic effects of caloric restriction are reflected in the in vivo adipocyte-enriched proteome of overweight/obese subjects. J Proteome Res, 2009. 8(12):5532-40.

38. Gilda JE, Gomes AV. Stain-Free total protein staining is a superior loading control to beta-actin for Western blots. Anal Biochem, 2013. 440(2):186-8.

39. Mutch DM, Pers TH, Temanni MR, Pelloux V, Marquez-Quinones A, Holst C et al. A distinct adipose tissue gene expression response to caloric restriction predicts 6-mo weight maintenance in obese subjects. Am J Clin Nutr, 2011. 94(6):1399-409.

40. Clarke JP, Mearow KM. Cell stress promotes the association of phosphorylated HspB1 with F-actin. PLoS One, 2013. 8(7):e68978.

41. Sreedharan R, Riordan M, Thullin G, Van Why S, Siegel NJ, Kashgarian M. The maximal cytoprotective function of the heat shock protein 27 is dependent on heat shock protein 70. Biochim Biophys Acta, 2011. 1813(1):129-35.

42. Sarto C, Binz PA, Mocarelli P. Heat shock proteins in human cancer. Electrophoresis, 2000. 21(6):121826.

43. Helenius A, Aebi M. Intracellular functions of N-linked glycans. Science, 2001. 291(5512):2364-9.

44. Jones FS, Jones PL. The tenascin family of ECM glycoproteins: structure, function, and regulation during embryonic development and tissue remodeling. Dev Dyn, 2000. 218(2):235-59.

45. Mosser DD, Caron AW, Bourget L, Meriin AB, Sherman MY, Morimoto RI, Massie B. The chaperone function of hsp70 is required for protection against stress-induced apoptosis. Mol Cell Biol, 2000. 20(19):7146-59.

46. Simar D, Jacques A, Caillaud C. Heat shock proteins induction reduces stress kinases activation, potentially improving insulin signalling in monocytes from obese subjects. Cell Stress Chaperones, 2012. 17(5):615-21. 
47. Dasu MR, Devaraj S, Park S, Jialal I. Increased toll-like receptor (TLR) activation and TLR ligands in recently diagnosed type 2 diabetic subjects. Diabetes Care, 2010. 33(4):861-8.

48. Renes J, Bouwman F, Noben JP, Evelo C, Robben J, Mariman E. Protein profiling of 3T3-L1 adipocyte differentiation and (tumor necrosis factor alpha-mediated) starvation. Cell Mol Life Sci, 2005. 62(4):492-503.

49. Kolehmainen M, Salopuro T, Schwab US, Kekalainen J, Kallio P, Laaksonen DE et al. Weight reduction modulates expression of genes involved in extracellular matrix and cell death: the GENOBIN study. Int J Obes (Lond), 2008. 32(2):292-303.

50. Mariman EC. Human biology of weight maintenance after weight loss. J Nutrigenet Nutrigenomics, 2012. 5(1):13-25.

51. Mariman EC. An adipobiological model for weight regain after weight loss. Adipobiology, 2011. 3(1):915.

52. Bouwman FG, Wang $P$, van Baak M, Saris WH, Mariman EC. Increased beta-oxidation with improved glucose uptake capacity in adipose tissue from obese after weight loss and maintenance. Obesity (Silver Spring), 2014. 22(3):819-27.

53. Maffei M, Halaas J, Ravussin E, Pratley RE, Lee GH, Zhang Y et al. Leptin levels in human and rodent: measurement of plasma leptin and ob RNA in obese and weight-reduced subjects. Nat Med, 1995. 1(11):1155-61. 
Supplementary Figures and Tables 


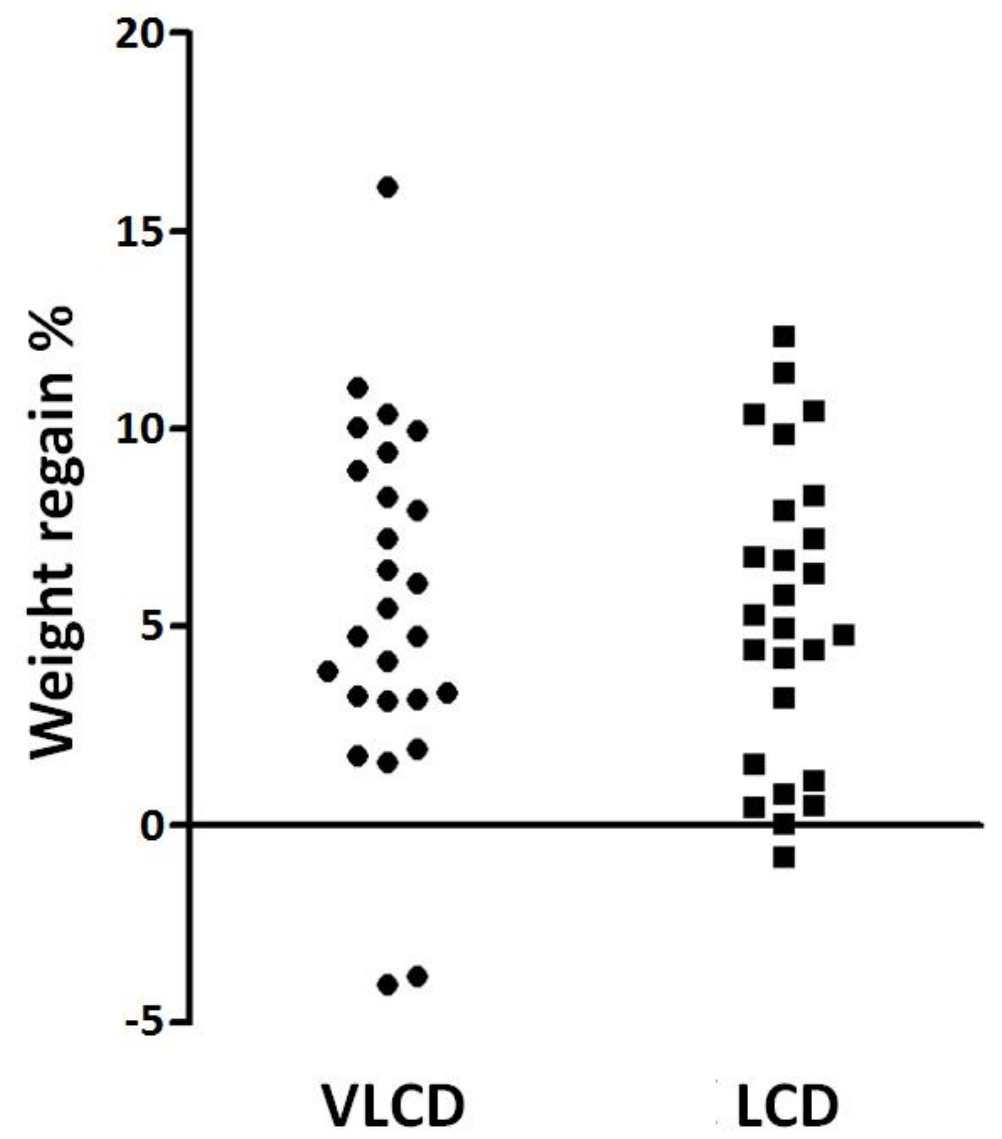

Supplement Figure 1: Plot of all individual weight regain percentages for VLCD and LCD. Weight regain percentage is calculated: ((weight after follow-up - weight after WS) $\div$ weight after WS) x 100\%. VLCD, very-low-calorie diet; LCD, lowcalorie diet. 
Supplement Table 1: Subject characteristics at study start, end of weight loss and end of weight stable.

\begin{tabular}{|c|c|c|c|c|c|c|}
\hline & \multicolumn{2}{|c|}{ Study start (T1) } & \multicolumn{2}{|c|}{ End of WL (T2) } & \multicolumn{2}{|c|}{ End of WS (T3) } \\
\hline & VLCD & LCD & VLCD & LCD & VLCD & LCD \\
\hline Sex (male/female) & $12 / 14$ & $13 / 14$ & & & & \\
\hline Age (years) & $50.4 \pm 1.5$ & $51.7 \pm 2.1$ & & & & \\
\hline Weight (kg) & $92.1 \pm 1.9$ & $92.8 \pm 2.0$ & $83.1 \pm 1.6$ & $84.6 \pm 2.0$ & $82.9 \pm 1.7$ & $84.5 \pm 2.0$ \\
\hline BMI $\left(\mathrm{kg} / \mathrm{m}^{2}\right)$ & $30.8 \pm 0.4$ & $31.5 \pm 0.5$ & $27.8 \pm 0.4$ & $28.7 \pm 0.5$ & $27.7 \pm 0.4$ & $28.7 \pm 0.5$ \\
\hline Hip circumference $(\mathrm{cm})$ & $111.0 \pm 1.1$ & $110.7 \pm 1.4$ & $105.0 \pm 1.0$ & $106.1 \pm 1.5$ & $104.8 \pm 1.0$ & $104.8 \pm 1.5^{\dagger}$ \\
\hline Waist circumference $(\mathrm{cm})$ & $101.3 \pm 1.6$ & $102.5 \pm 2.1$ & $93.5 \pm 1.4$ & $95.2 \pm 1.9$ & $94.6 \pm 1.4$ & $94.4 \pm 2.1$ \\
\hline Body fat (\%) & $39.5 \pm 1.6$ & $40.6 \pm 1.9$ & $34.8 \pm 2.0$ & $34.7 \pm 2.2$ & $33.7 \pm 2.0+\dagger$ & $34.1 \pm 2.3^{\dagger}$ \\
\hline Body fat (kg) & $35.7 \pm 1.2$ & $36.8 \pm 1.9$ & $28.4 \pm 1.5$ & $29.2 \pm 2.1$ & $27.3 \pm 1.4+\dagger$ & $28.7 \pm 2.1+$ \\
\hline Fat free mass $(\mathrm{kg})$ & $55.7 \pm 2.4$ & $55.5 \pm 2.3$ & $54.2 \pm 2.3$ & $55.0 \pm 2.3$ & $54.9 \pm 2.4+\dagger$ & $55.3 \pm 2.3$ \\
\hline
\end{tabular}

Values are mean $\pm S E M .{ }^{*} P \leq 0.05$, independent T-test, comparison between VLCD and LCD at study start, end of WL and end of WS. No significant differences were observed between the LCD and VLCD at the study start. $+P \leq 0.05,+\dagger<0.01$, dependent T-test, comparison between end of WL vs. end of WS. WL, weight loss; WS, weight stable; VLCD, very-lowcalorie diet; LCD, low-calorie diet; BMI, body mass index. 
Supplement Table 2: List of the 107 candidate stress-related genes used for the analysis.

\begin{tabular}{|c|c|c|c|}
\hline Gene ID & Gene name & Gene \# & Chromosome \\
\hline ACTB & actin, beta & 60 & 7 \\
\hline ACTG1 & actin gamma 1 & 71 & 17 \\
\hline AHSA2 & AHA1, activator of heat shock $90 \mathrm{kDa}$ protein ATPase homolog 2 (yeast) & 130872 & 2 \\
\hline AKR1B1 & aldo-keto reductase family 1 , member B1 (aldose reductase) & 231 & 7 \\
\hline $\mathrm{ALDH} 2$ & aldehyde dehydrogenase 2 family (mitochondrial) & 217 & 12 \\
\hline ALDOA & aldolase $A$, fructose-bisphosphate & 226 & 16 \\
\hline ANXA2 & annexin $\mathrm{A} 2$ & 302 & 15 \\
\hline ANXA5 & annexin $\mathrm{A} 5$ & 308 & 4 \\
\hline AQP1 & aquaporin 1 (Colton blood group) & 358 & 7 \\
\hline ARHGDIA & Rho GDP dissociation inhibitor (GDI) alpha & 396 & 17 \\
\hline ATF3 & activating transcription factor 3 & 467 & 1 \\
\hline ATF4 & activating transcription factor 4 & 468 & 22 \\
\hline ATP2B4 & ATPase, $\mathrm{Ca}++$ transporting, plasma membrane 4 & 493 & 1 \\
\hline ATP5B & ATP synthase, $\mathrm{H}+$ transporting, mitochondrial F1 complex, beta polypeptide & 506 & 12 \\
\hline BCLAF1 & BCL2-associated transcription factor 1 & 9774 & 6 \\
\hline $\mathrm{C} 5$ & complement component 5 & 727 & 9 \\
\hline CALR & Calreticulin & 811 & 19 \\
\hline CFL1 & cofilin 1 (non-muscle) & 1072 & 11 \\
\hline CHORDC1 & cysteine and histidine-rich domain (CHORD) containing 1 & 26973 & 11 \\
\hline CLIC1 & chloride intracellular channel 1 & 1192 & 6 \\
\hline CRHBP & corticotropin releasing hormone binding protein & 1393 & 5 \\
\hline CRYAB & crystallin, alpha B & 1410 & 11 \\
\hline CTSD & cathepsin D & 1509 & 11 \\
\hline DDIT3 & DNA-damage-inducible transcript 3 & 1649 & 12 \\
\hline DNAJC3 & DnaJ (Hsp40) homolog, subfamily C, member 3 & 5611 & 13 \\
\hline DUSP10 & dual specificity phosphatase 10 & 11221 & 1 \\
\hline EDEM1 & ER degradation enhancer, mannosidase alpha-like 1 & 9695 & 3 \\
\hline EGFR & epidermal growth factor receptor & 1956 & 7 \\
\hline EIF2AK1 & eukaryotic translation initiation factor 2 -alpha kinase 1 & 27102 & 7 \\
\hline EIF2B3 & eukaryotic translation initiation factor $2 \mathrm{~B}$, subunit 3 gamma, $58 \mathrm{kDa}$ & 8891 & 1 \\
\hline EIF2S1 & eukaryotic translation initiation factor 2 , subunit 1 alpha, 35kDa & 1965 & 14 \\
\hline EIF5A & eukaryotic translation initiation factor $5 \mathrm{~A}$ & 1984 & 17 \\
\hline ENO1 & enolase 1, (alpha) & 2023 & 1 \\
\hline ERRFI1 & ERBB receptor feedback inhibitor 1 & 54206 & 1 \\
\hline GADD45B & growth arrest and DNA-damage-inducible, beta & 4616 & 19 \\
\hline GADD45G & growth arrest and DNA-damage-inducible, gamma & 10912 & 9 \\
\hline GATA4 & GATA binding protein 4 & 2626 & 8 \\
\hline GATA6 & GATA binding protein 6 & 2627 & 18 \\
\hline GPR132 & G protein-coupled receptor 132 & 29933 & 14 \\
\hline GSTP1 & glutathione S-transferase pi 1 & 2950 & 11 \\
\hline HEY2 & hes-related family bHLH transcription factor with YRPW motif 2 & 23493 & 6 \\
\hline HILPDA & hypoxia inducible lipid droplet-associated & 29923 & 7 \\
\hline HSF2 & heat shock transcription factor 2 & 3298 & 6 \\
\hline HSF4 & heat shock transcription factor 4 & 3299 & 16 \\
\hline HSP90AB4P & heat shock protein 90kDa alpha (cytosolic), class B member 4, pseudogene & 664618 & 15 \\
\hline HSP9OB2P & heat shock protein 90kDa beta (Grp94), member 2, pseudogene & 7190 & 15 \\
\hline HSPA5 & heat shock 70kDa protein 5 (glucose-regulated protein, 78kDa) & 3309 & 9 \\
\hline HSPA8 & heat shock 70kDa protein 8 & 3312 & 11 \\
\hline HSPB1 & heat shock $27 \mathrm{kDa}$ protein 1 & 3315 & 7 \\
\hline HSPB11 & heat shock protein family B (small), member 11 & 51668 & 1 \\
\hline HSPB6 & heat shock protein, alpha-crystallin-related, B6 & 126393 & 19 \\
\hline HSPB8 & heat shock $22 \mathrm{kDa}$ protein 8 & 26353 & 12 \\
\hline HSPD1 & heat shock 60kDa protein 1 (chaperonin) & 3329 & 2 \\
\hline HYOU1 & hypoxia up-regulated 1 & 10525 & 11 \\
\hline IDO1 & indoleamine 2,3-dioxygenase 1 & 3620 & 8 \\
\hline KLF15 & Kruppel-like factor 15 & 28999 & 3 \\
\hline KLF2 & Kruppel-like factor 2 & 10365 & 19 \\
\hline KRT8 & keratin 8, type II & 3856 & 12 \\
\hline LGALS1 & lectin, galactoside-binding, soluble, 1 & 3956 & 22 \\
\hline LMNA & $\operatorname{lamin} \mathrm{A} / \mathrm{C}$ & 4000 & 1 \\
\hline MAP4K1 & mitogen-activated protein kinase kinase kinase kinase 1 & 11184 & 19 \\
\hline MAPK11 & mitogen-activated protein kinase 11 & 5600 & 22 \\
\hline MAPK13 & mitogen-activated protein kinase 13 & 5603 & 6 \\
\hline MAPK7 & mitogen-activated protein kinase 7 & 5598 & 17 \\
\hline MAPK8 & mitogen-activated protein kinase 8 & 5599 & 10 \\
\hline
\end{tabular}




\begin{tabular}{|c|c|c|c|}
\hline MAPK9 & mitogen-activated protein kinase 9 & 5601 & 5 \\
\hline MAPKAPK3 & mitogen-activated protein kinase-activated protein kinase 3 & 7867 & 3 \\
\hline MEF2C & myocyte enhancer factor $2 \mathrm{C}$ & 4208 & 5 \\
\hline MUC1 & mucin 1 , cell surface associated & 4582 & 1 \\
\hline NDRG4 & NDRG family member 4 & 65009 & 16 \\
\hline NME2 & NME/NM23 nucleoside diphosphate kinase 2 & 4831 & 17 \\
\hline NPM1 & nucleophosmin (nucleolar phosphoprotein B23, numatrin) & 4869 & 5 \\
\hline OMA1 & OMA1 zinc metallopeptidase & 115209 & 1 \\
\hline $\mathrm{P} 4 \mathrm{HB}$ & prolyl 4-hydroxylase, beta polypeptide & 5034 & 17 \\
\hline PGK1 & phosphoglycerate kinase 1 & 5230 & $x$ \\
\hline PHB & Prohibitin & 5245 & 17 \\
\hline PKM & pyruvate kinase, muscle & 5315 & 15 \\
\hline PPIA & peptidylprolyl isomerase A (cyclophilin A) & 5478 & 7 \\
\hline PRDX6 & peroxiredoxin 6 & 9588 & 1 \\
\hline PRKAA2 & protein kinase, AMP-activated, alpha 2 catalytic subunit & 5563 & 1 \\
\hline PSME1 & proteasome (prosome, macropain) activator subunit 1 (PA28 alpha) & 5720 & 14 \\
\hline PTEN & phosphatase and tensin homolog & 5728 & 10 \\
\hline PTK2B & protein tyrosine kinase 2 beta & 2185 & 8 \\
\hline RGS14 & regulator of G-protein signaling 14 & 10636 & 5 \\
\hline RPS6KA1 & ribosomal protein $\mathrm{S} 6$ kinase, 90kDa, polypeptide 1 & 6195 & 1 \\
\hline RPS6KA3 & ribosomal protein $\mathrm{S} 6$ kinase, 90kDa, polypeptide 3 & 6197 & $x$ \\
\hline RTN3 & reticulon 3 & 10313 & 11 \\
\hline SGK1 & serum/glucocorticoid regulated kinase 1 & 6446 & 6 \\
\hline SNN & Stannin & 8303 & 16 \\
\hline SQSTM1 & sequestosome 1 & 8878 & 5 \\
\hline STC2 & stanniocalcin 2 & 8614 & 5 \\
\hline STIP1 & stress-induced phosphoprotein 1 & 10963 & 11 \\
\hline STK39 & serine threonine kinase 39 & 27347 & 2 \\
\hline STMN1 & stathmin 1 & 3925 & 1 \\
\hline TAOK2 & TAO kinase 2 & 9344 & 16 \\
\hline TCAP & titin-cap & 8557 & 17 \\
\hline TENM1 & teneurin transmembrane protein 1 & 10178 & $x$ \\
\hline TMEM204 & transmembrane protein 204 & 79652 & 16 \\
\hline TP53I11 & tumor protein p53 inducible protein 11 & 9537 & 11 \\
\hline TP53INP1 & tumor protein p53 inducible nuclear protein 1 & 94241 & 8 \\
\hline TP53TG1 & TP53 target 1 (non-protein coding) & 11257 & 7 \\
\hline TPI1 & triosephosphate isomerase 1 & 7167 & 12 \\
\hline TPM3 & tropomyosin 3 & 7170 & 1 \\
\hline TRAF2 & TNF receptor-associated factor 2 & 7186 & 9 \\
\hline TUBB & tubulin, beta class I & 203068 & 6 \\
\hline UCN2 & urocortin 2 & 90226 & 3 \\
\hline ZAK & sterile alpha motif and leucine zipper containing kinase AZK & 51776 & 2 \\
\hline
\end{tabular}

Sixteen genes were specifically related to endoplasmic reticulum stress as previously reported $(18,19)$, twenty-four candidate stress-related genes were selected based on the frequently detected proteins in subcutaneous adipose tissue(26) and sixty-seven were genes with the term "response to stress" in the Gene Ontology Biological Process (GO-BP) description. 
Supplement Table 3: List of the 22 candidate stress-related proteins used for the analysis.

\begin{tabular}{lll}
\hline Protein ID & Protein name & MW (kDa) \\
\hline P10809 & 60 kDa heat shock protein, mitochondrial [OS=Homo sapiens] & 61.016 \\
P11021 & 78 kDa glucose-regulated protein [OS=Homo sapiens] & 72.288 \\
P60709 & Actin, cytoplasmic 1 [OS=Homo sapiens] & 41.710 \\
P05091 & Aldehyde dehydrogenase, mitochondrial [OS=Homo sapiens] & 56.346 \\
P02511 & Alpha-crystallin B chain [OS=Homo sapiens] & 20.146 \\
P08758 & Annexin A5 [OS=Homo sapiens] & 35.914 \\
P06576 & ATP synthase subunit beta, mitochondrial [OS=Homo sapiens] & 56.525 \\
P27797 & Calreticulin [OS=Homo sapiens] & 48.112 \\
P07339 & Cathepsin D [OS=Homo sapiens] & 44.524 \\
O00299 & Chloride intracellular channel protein 1 [OS=Homo sapiens] & 26.906 \\
P09382 & Galectin-1 [OS=Homo sapiens] & 14.706 \\
P04792 & Heat shock protein beta-1 [OS=Homo sapiens] & 22.768 \\
O14558 & Heat shock protein beta-6 [OS=Homo sapiens] & 17.125 \\
P06748 & Nucleophosmin [OS=Homo sapiens] & 32.555 \\
P62937 & peptidyl-prolyl cis-trans isomerase A [OS=Homo sapiens] & 18.001 \\
P30041 & Peroxiredoxin-6 [OS=Homo sapiens] & 25.019 \\
P00558 & Phosphoglycerate kinase 1 [OS=Homo sapiens] & 44.586 \\
P02545 & Prelamin-A/C [OS=Homo sapiens] & 74.095 \\
P35232 & Prohibitin [OS=Homo sapiens] & 29.786 \\
P07237 & Protein disulfide-isomerase [OS=Homo sapiens] & 57.081 \\
P14618 & Pyruvate kinase PKM [OS=Homo sapiens] & 57.900 \\
P60174 & Triosephosphate isomerase [OS=Homo sapiens] & 30.772 \\
\hline
\end{tabular}


Supplement Table 4: All significant correlations between the weight regain percentage and the fold changes in gene expression levels during the weight loss, weight stable and dietary intervention period.

\begin{tabular}{|c|c|c|c|c|c|c|c|}
\hline \multirow[t]{2}{*}{ Gene ID } & \multirow[t]{2}{*}{ Gene name } & \multicolumn{3}{|l|}{ VLCD } & \multicolumn{3}{|l|}{ LCD } \\
\hline & & WL & WS & DI & WL & WS & DI \\
\hline ACTB & Actin, beta & & 0.562 & & & & \\
\hline AKR1B1 & Aldo-keto reductase family 1 , member B1 & & 0.453 & & & & \\
\hline ALDH2 & Aldehyde dehydrogenase 2 family & 0.466 & & & & & 0.520 \\
\hline ALDOA & Aldolase $\mathrm{A}$, fructose-bisphosphate & & 0.552 & 0.458 & 0.590 & & 0.473 \\
\hline ANXA5 & Annexin A5 & 0.484 & & 0.566 & & & \\
\hline ARHGDIA & Rho GDP dissociation inhibitor (GDI) alpha & & 0.507 & 0.436 & & & \\
\hline ATP5B & ATP synthase, $\mathrm{H}+$ transporting, mitochondrial $\mathrm{F} 1$ complex, beta polypeptide & & 0.478 & 0.488 & & & \\
\hline BCLAF1 & BCL2-associated transcription factor 1 & & -0.433 & & & 0.441 & \\
\hline C5 & Complement component 5 & & -0.525 & & & & \\
\hline CALR & Calreticulin & & 0.415 & 0.428 & & & \\
\hline CFL1 & Cofilin 1 & & 0.607 & 0.455 & & & \\
\hline CLIC1 & Chloride intracellular channel 1 & & 0.431 & & & & \\
\hline CRHBP & Corticotropin releasing hormone binding protein & & & -0.518 & & & \\
\hline CRYAB & Crystallin, alpha B & 0.586 & & 0.671 & & & \\
\hline DUSP10 & Dual specificity phosphatase 10 & & & 0.424 & & & \\
\hline EIF2AK1 & Eukaryotic translation initiation factor 2 -alpha kinase 1 & & & 0.456 & & & \\
\hline EIF2B3 & Eukaryotic translation initiation factor $2 \mathrm{~B}$, subunit 3 gamma, $58 \mathrm{kDa}$ & & & & 0.454 & & \\
\hline ENO1 & Enolase 1, alpha & & 0.710 & 0.684 & & & \\
\hline GSTP1 & Glutathione S-transferase pi 1 & 0.444 & & 0.597 & & & \\
\hline HSF2 & Heat shock transcription factor 2 & & & & & 0.414 & \\
\hline HSP90B2P & Heat shock protein $90 \mathrm{kDa}$ beta, member 2 , pseudogene & & 0.438 & & & 0.416 & \\
\hline HSPA5 & Heat shock 70kDa protein 5 & & 0.457 & & & & \\
\hline HSPA8 & Heat shock 70kDa protein 8 & & 0.519 & & & & \\
\hline HSPB1 & Heat shock 27kDa protein 1 & & 0.470 & & & & \\
\hline HSPB8 & Heat shock $22 \mathrm{kDa}$ protein 8 & & & 0.420 & & & \\
\hline HSPD1 & Heat shock 60kDa protein 1 (chaperonin) & & & & 0.448 & & \\
\hline HYOU1 & Hypoxia up-regulated 1 & & 0.443 & & & -0.445 & \\
\hline KLF15 & Kruppel-like factor 15 & & & & & 0.431 & \\
\hline LGALS1 & Lectin, galactoside-binding, soluble, 1 & & & 0.705 & & & \\
\hline MAPK13 & Mitogen-activated protein kinase 13 & & 0.586 & & & & \\
\hline MAPK7 & Mitogen-activated protein kinase 7 & & & & 0.445 & & \\
\hline MAPK8 & Mitogen-activated protein kinase 8 & & -0.477 & & & & \\
\hline MAPKAPK3 & Mitogen-activated protein kinase-activated protein kinase 3 & & 0.519 & & & & \\
\hline MEF2C & Myocyte enhancer factor $2 \mathrm{C}$ & & -0.499 & -0.454 & & & \\
\hline MUC1 & Mucin 1 , cell surface associated & & & & & -0.423 & \\
\hline NME2 & NME/NM23 nucleoside diphosphate kinase 2 & & & & & & -0.503 \\
\hline OMA1 & OMA1 zinc metallopeptidase & & -0.443 & & & & \\
\hline PGK1 & Phosphoglycerate kinase 1 & -0.434 & 0.655 & 0.417 & & & \\
\hline PHB & Prohibitin & & & 0.528 & & & \\
\hline
\end{tabular}


PSME1 Proteasome (prosome, macropain) activator subunit 1

\subsection{3}

(1)

Phosphatase and tensin homolog

Protein tyrosine kinase 2 beta

$-0.547$

$-0.426$

PTEN

Regulator of G-protein signaling 14

$-0.506$

0.658

RGS14

Ribosomal protein 56 kinase, 90kDa, polypeptide 1

0.500
0.643

Reticulon 3

$\begin{array}{ll}\text { SNN } & \text { Stannin } \\ \text { SQSTM1 } & \text { Sequestosome 1 }\end{array}$

0.459

TPI1 Triosephosphate isomerase 1

UCN2 Urocortin 2

Sterile alpha motif and leucine zipper containing kinase AZK

0.606

0.436

$-0.444$

Correlation coefficients $(r)$ between weight regain and the fold change (FC) of gene expression during weight loss (T1-T2), weight stable (T2-T3) and dietary intervention (T1-T3). Weight regain percentage is calculated: ((weight after follow-up - weight after WS) $\div$ weight after WS) $\times 100 \%$. Only Pearson R's correlations with a P-value $\leq 0.05$ are depicted in this table. VLCD, very-lowcalorie diet; LCD, low-calorie diet; WL, weight loss; WS, weight stable; DI, dietary intervention. 


\section{Chapter 6}

\section{Relation between stress- and ECM-related genes and their effect on weight regain}




\begin{abstract}
Background/Objective: The adipose tissue plays a central role in the risk for weight regain. During a negative energy balance, the volume of adipocytes decreases due to loss of triglycerides which may lead to stress due to the misfit between the cell contents and the surrounding extracellular matrix (ECM) (1).This stress can be resolved by remodeling of the ECM or the re-storing of triglycerides within the adipocytes $(1,2)$. Possibly, there is a connection between stress-related and ECM-related genes, which is associated with weight regain. Our main objective was to investigate the existence of such a connection.
\end{abstract}

Methods: In this randomized controlled trial, twenty-six healthy overweight/obese participants followed a 5-week very-low-calorie diet $(500 \mathrm{kcal} / \mathrm{d})$ with subsequently a 4-week weight stable diet (WS period), and an uncontrolled 9-month follow-up. Abdominal subcutaneous adipose tissue biopsies were collected for microarray analysis. We performed correlation and interaction analysis with the weight regain percentage using two gene sets which we previously defined as 'stressrelated' and as 'ECM-related' genes.

Results: During the WS phase, a co-expression network of 8 stress- and 15 ECM-related genes correlating with weight regain could be constructed, which links to the biological processes on leukocyte-activity, ECM remodelling, actin cytoskeleton organisation and glucose handling. Interaction analysis between stress- and ECM-related genes revealed several gene combinations that were highly related to the weight regain percentage. In particular, the epidermal growth factor signaling pathway was identified as strongly influencing the risk of weight regain, possibly through interaction with actinin alpha-1 (ACTN1), a component of stress fibers, and with integrin beta 4 (ITGB4), cystatin C (CST3) and laminin alpha-3 (LAMA3).

Conclusion: Our present findings indicate the importance of the connection between stress- and ECM-related genes in the risk for weight regain 


\section{Introduction}

The prevalence of overweight and obesity has increased to epidemic proportions and the related comorbidities are major threats to human health (3). Achieving $5-10 \%$ weight loss by dietary intervention, increased physical activity, or pharmacological and/or surgical treatment produces positive health outcomes in overweight or obese people $(4,5)$. However, long-term weight loss maintenance has been proven to be difficult. In general, only $20 \%$ of the people are successful in maintaining weight loss $(6,7)$. After weight regain, the risk for metabolic complications appears higher than at the start of the weight loss period $(8,9)$. Therefore, it is important to understand the mechanisms influencing the risk for weight regain.

The adipose tissue plays a central role in the risk for weight regain. It was previously hypothesized that the volume of adipocytes decreases due to loss of triglycerides during a negative energy balance creating stress between the cell contents and the surrounding extracellular matrix (ECM) (1). This stress can be resolved by remodeling of the ECM or the re-storing of triglycerides within the adipocytes $(1,2)$. It suggests that cellular stress accumulated in adipocytes during a negative energy balance is a driving force behind the risk for weight regain. In line, we have shown higher cellular stress in the subcutaneous adipose tissue of participants regaining their lost weight compared to the participants maintaining weight (10). Furthermore, the risk for weight regain is related to changes in the expression of certain stress-related genes during the first four weeks after returning to a weight stable situation. These stress-related genes are involved in actin filament dynamics, glucose handling and nutrient sensing (11). Interestingly, after returning to energy balance changes in expression of certain ECM-related genes are also correlated to weight regain (12). Based on the proposed model, it is possible that there is a connection between stress-related genes and ECM-related genes associated with weight regain. In the present study, our main objective was to expand on our previous studies to investigate the existence and relevance of such a connection. Therefore, we performed correlation and interaction analysis using two gene sets which we previously defined as 'stress-related' and as 'ECM-related' genes during the weight loss (13), the weight stabilisation (14) and the dietary intervention (DI) phase of a very-low-calorie diet (VLCD).

\section{Methods}

\section{Participants and study design}

The present study was performed in a sub-cohort of our larger clinical trial as described previously (15), in which only the VLCD intervention was selected. Thirty-one overweight and obese (BMI 27-36 $\mathrm{kg} / \mathrm{m}^{2}$ ) participants were included in the present study. All participants gave their written informed consent before participation in the study. The study was performed according to the declaration of Helsinki and was approved by the Medical Ethics Committee of Maastricht University Medical Centre.

In short, participants had to remain weight stable (weight change $<3.0 \mathrm{~kg}$ ) two months prior to the start of the study. Thereafter, study participants underwent a 5 -week VLCD (500 kcal/day), targeting $10 \%$ weight loss (WL period, T1-T2) by consuming three meal replacements per day (Modifast; Nutrition et Santé Benelux), and a subsequent 4-week weight maintenance diet (WS period, T2-T3) based on their individual energy requirements (Figure 1). This WS period was designed to investigate the effect of weight loss without the interfering effect of a pronounced negative energy balance. The WL and WS period taken together was named the dietary intervention (DI, T1-T3). After the WS 
period, participant's body weight and blood pressure were monitored for 9 months (follow-up, T3T4). During this follow-up, participants did not receive advice on monitoring and limiting food intake to mimic non-restricted free-living conditions. This trial is registered with www.clinicaltrials.gov: as NCT01559415.

\begin{tabular}{|c|c|c|}
\hline Weight loss & Weight stable & Follow-up \\
\hline VLCD 5 weeks & 4 weeks & 9 months \\
\hline
\end{tabular}

Figure 1: Schematic overview of the study design. Anthropometry, adipose tissue biopsies and proteomics were performed at the start of the study (T1), at the end of the weight loss period (T2), weight stable period (T3), and follow-up (T4).

Transcriptomics were performed at $\mathrm{T} 1, \mathrm{~T} 2$ and $\mathrm{T} 3$. The dietary intervention period (DI) is the weight loss period and weight stable period taken together. VLCD, very-low-calorie diet $(n=26)$.

At baseline, end of WL, end of WS and end of follow-up, body weight, blood pressure and, waist and hip circumferences were measured, body composition was determined, fasting blood samples were drawn, and abdominal subcutaneous adipose tissue biopsies were collected as described previously (15). As defined in our previous studies, the weight regain percentage (WR\%) $=(($ weight after followup T4 - weight after WS T3) $\div$ weight after WS T3) x 100\%.

\section{Adipose tissue biopsy}

Abdominal subcutaneous adipose tissue biopsies were obtained by needle biopsy under local anaesthesia (2\% lidocaine, Fresenius Kabi) after an overnight fast at T1, T2 T3, and T4. Tissue was immediately rinsed in sterile saline, frozen in liquid nitrogen and stored at $-80^{\circ} \mathrm{C}$ until RNA and protein isolation.

\section{Adipose tissue RNA isolation and microarray analysis}

For RNA isolation, adipose tissue samples from T1, T2 and T3 were used. Total RNA was extracted from approx. $150 \mathrm{mg}$ frozen adipose tissue using TRIzol reagent (Invitrogen, Breda, The Netherlands). Total RNA (100 ng per sample) was labeled by Whole-Transcript Sense Target Assay and hybridized to human whole-genome Affymetrix Gene 1.1 ST arrays targeting 19654 unique genes (Affymetrix). Sample labeling, hybridization to chips and image scanning was performed according to manufacturer's instructions. Microarray signals were normalized using robust multichip average (RMA). Genes with normalized signals $>20$ on at least 15 arrays were defined as expressed (11532 genes). Significant differences of individual genes were tested using the limma R library (16). P-values were adjusted using false discovery rate (FDR) (17) and an adjusted P-value (q-value) $\leq 0.05$ was considered significant. Array data have been submitted to the Gene Expression Omnibus for a larger cohort $(n=53)$ (number GSE77962) while here we show the results of a sub-group. 


\section{Gene selection}

Based on gene ontology, a set of 107 'stress-related' and a set of 277 'ECM-related' genes (Supplement Table 1) were selected, which were the same as in the previous analyses $(11,12)$

\section{Data analysis}

The gene expression change was used to study the relationship between the gene changes and the weight regain percentage. We calculated the fold change $(F C)$ of the expression of each gene during WL, WS and DI, respectively.

\section{Correlation analysis}

Correlations were made between stress-related and ECM-related genes during VLCD WL, WS and DI using the Pearson correlation coefficient. For this, only genes were included which were found to be correlated with the WR\% in previous analyses $(11,12)$. The correlations between stress- and ECMrelated genes were entered in the MetScape 3 App (18) for the software platform Cytoscape (14) to make correlation based networks during the WL, WS and DI phase. In principle, genes were included that significantly correlated with $|r| \geq 0.600$ and $\mathrm{P} \leq 0.001$ (unless indicated otherwise).

\section{Interaction analysis}

Linear regression model $y=f(x 1, x 2, x 1 \times x 2)$ was calculated for the effect of interaction between change in expression of $x 1$ : each gene in the stress-gene set and $x 2$ : each gene in the ECM-gene set, at $\mathrm{WL}, \mathrm{WS}$ and $\mathrm{DI}$, respectively, for $\mathrm{y}$ : the weight regain percentage. The analysis was run in $\mathrm{R}$ version 3.3.1, a gene pair with P-value of the interaction $\leq 0.001$ was taken as significant interaction pair.

\section{Other statistical analysis}

Data are presented as mean $\pm S E M$. Variation in the number of participants between analyses is due to the exclusion of participants with missing data. Dependent T-test was carried out to determine possible effects over time. For all statistical tests $\mathrm{P} \leq 0.05$ was considered to be statistically significant unless indicated otherwise. All variables were checked for normal distribution, and variables with a skewed distribution were LN-transformed to satisfy conditions of normality. Extreme outliers (values higher than $3 x$ interquartile range calculated with SPSS) influencing the data were removed during statistical analyses. These analyses were done using SPSS 20.0 for Windows (SPSS Inc, Chicago, IL).

\section{Results}

\section{Study population}

Three participants withdrew from the study during the dietary intervention (T1-T3) due to personal circumstances, for one participant we could not collect enough biopsy material and for one participant the gene expression results deviated strongly from the others and were therefore excluded. Characteristics of the remaining twenty-six participants at study start (T1), end of WL (T2), end of WS (T3) and end of follow-up (T4) are displayed in Table 1. Body weight, BMI, hip and waist circumference, body fat and fat free mass significantly decreased during weight loss and remained rather stable during the WS period. The average weight regain percentage was $5.4 \pm 4.5 \%$. 
Table 1: Subject characteristics at study start, end of weight loss and end of weight stable.

\begin{tabular}{|c|c|c|c|c|}
\hline Gene ID & Study start (T1) & End of WL (T2) & End of WS (T3) & End of follow-up (T4) \\
\hline Sex (male/female) & $12 / 14$ & & & \\
\hline Age (years) & $50.4 \pm 1.5$ & & & \\
\hline Weight (kg) & $92.1 \pm 1.9$ & $83.1 \pm 1.6^{* * *}$ & $82.9 \pm 1.7$ & $87.7 \pm 2.0^{* * *}$ \\
\hline $\mathrm{BMI}\left(\mathrm{kg} / \mathrm{m}^{2}\right)$ & $30.8 \pm 0.4$ & $27.8 \pm 0.4^{* * *}$ & $27.7 \pm 0.4$ & $29.1 \pm 0.5^{* * *}$ \\
\hline Hip circumference $(\mathrm{cm})$ & $111.0 \pm 1.1$ & $105.0 \pm 1.0^{* * *}$ & $104.8 \pm 1.0$ & $105.4 \pm 1.4$ \\
\hline Waist circumference $(\mathrm{cm})$ & $101.3 \pm 1.6$ & $93.5 \pm 1.4^{* * *}$ & $94.6 \pm 1.4$ & $97.6 \pm 1.7^{* * *}$ \\
\hline Body fat (\%) & $39.5 \pm 1.6$ & $34.8 \pm 2.0^{* * *}$ & $33.7 \pm 2.0^{* *}$ & $36.0 \pm 1.9 * * *$ \\
\hline Body fat $(\mathrm{kg})$ & $35.7 \pm 1.2$ & $28.4 \pm 1.5^{* * *}$ & $27.3 \pm 1.4^{* *}$ & $31.0 \pm 1.6^{* * *}$ \\
\hline Fat free mass $(\mathrm{kg})$ & $55.7 \pm 2.4$ & $54.2 \pm 2.3^{* * *}$ & $54.9 \pm 2.4^{* *}$ & $55.9 \pm 2.4^{*}$ \\
\hline
\end{tabular}

\section{Co-expression between stress- and ECM-related genes}

Stress- and ECM-related genes were first filtered from the sets based on significant correlation $(P \leq 0.05)$ with the WR\% as described previously $(11,12)$. In total, 124 genes met this criteria which were passed into the co-expression analysis: 28 genes during WL, 62 genes during WS and 34 genes during DI. 286 strong correlations $(|r| \geq 0.600 ; \mathrm{P} \leq 0.001)$ were found between the stress gene set and the ECM gene set. Interestingly, there was a large difference in the amount of strong correlations found between stress and ECM genes for the different phases: 18 during WL, 243 during WS and 25 during DI. Although for the analyses in the WS period more genes were used compared to the WL and DI phase, this cannot fully explain the large difference in correlations. Only 26 stress genes and $28 \mathrm{ECM}$ genes are responsible for the 243 correlations during WS, indicating that these genes have multiple correlations with other genes (Table 2). In fact, there are 12 stress genes that each correlated with at least $10 \mathrm{ECM}$ genes and $16 \mathrm{ECM}$ genes that each correlated with at least 10 stress genes. These results indicated that stress- and ECM-related genes were co-expressed especially during the weight stabilization period, not only as single gene pairs but rather in a complex manner.

Table 2: The number of correlations between stress-and ECM-related genes during the WS phase.

\begin{tabular}{|c|c|c|c|}
\hline Stress genes & $\begin{array}{l}\text { Connections to } \\
\text { ECM genes }\end{array}$ & ECM genes & $\begin{array}{l}\text { Connections to } \\
\text { stress genes }\end{array}$ \\
\hline CFL1 & 21 & PPIB & 15 \\
\hline PKM & 21 & CAPN1 & 15 \\
\hline RPS6KA1 & 18 & CTSB & 14 \\
\hline CALR & 17 & ITGA3 & 14 \\
\hline ZAK & 17 & COLGALT1 & 13 \\
\hline ENO1 & 13 & ACTN1 & 13 \\
\hline MAPK13 & 16 & EMILIN2 & 12 \\
\hline MAPKAРКЗ & 16 & PDIA4 & 12 \\
\hline ARHGDIA & 11 & SPARCL1 & 12 \\
\hline AKR1B1 & 11 & $\mathrm{ECM} 2$ & 11 \\
\hline ALDOA & 9 & ITGAX & 11 \\
\hline HYOU1 & 11 & ITGA5 & 10 \\
\hline PGK1 & 10 & PLOD1 & 10 \\
\hline CLIC1 & 9 & NCSTN & 10 \\
\hline PTK2B & 7 & DMD & 10 \\
\hline ACTB & 7 & ITGAM & 10 \\
\hline HSPA5 & 6 & ITGB5 & 8 \\
\hline MAPK8 & 6 & SPINT1 & 8 \\
\hline MEF2C & 3 & ITGAL & 8 \\
\hline OMA1 & 4 & CTSS & 6 \\
\hline RGS14 & 2 & ITGB2 & 6 \\
\hline HSPA8 & 2 & PLOD2 & 5 \\
\hline HSPB1 & 2 & KDR & 3 \\
\hline PTEN & 2 & VIT & 2 \\
\hline BCLAF1 & 1 & GPC1 & 2 \\
\hline \multirow[t]{3}{*}{ C5 } & 1 & ITGB3 & 1 \\
\hline & & ICAM3 & 1 \\
\hline & & NCAM1 & 1 \\
\hline
\end{tabular}

Pearson correlations with $|r| \geq 0.600$ and $\mathrm{P} \leq 0.001$. ECM, extracellular matrix; WS, weight stable. 
Next, we wanted to see if the correlation genes formed a specific network. With the correlation criteria $|r| \geq 0.600$ and $P \leq 0.001$, we observed a network during all phases (Supplement Figure 1). For the WS phase more stringent criteria $(|r| \geq 0.800 ; \mathrm{P} \leq 0.001)$ were used to be able to detect the most important gene correlations. The network consists of 8 stress-related genes and 15 ECM-related genes. All genes were positively correlated with each other as well as with the WR\%, except dystrophin (DMD) which was negatively correlated with integrin subunit alpha M (ITGAM) and weight regain (Figure 2). Ten out of the 23 genes are strongly correlated with weight regain $(P \leq 0.01)$. The previously detected correlation network of integrin genes ITGAL, ITGAM, ITGAX and ITGB2 (12) was found to correlate strongly with the gene for ribosomal protein S6 kinase A1 (RPS6KA1). The latter gene was previously identified as part of a network based on gene regulatory and biochemical interactions together with the genes for aldolase $A$ (ALDOA), enolase 1 (ENO1), cofilin 1 (CFL1) and mitogen-activated protein kinase 13 (MAPK13) (11). These genes turn out to be part of the stressECM network as well. CFL1 strongly correlates with 11 other genes of which 4 from the stress and 7 from the ECM gene set. Also, pyruvate kinase (PKM) seems to play a central role with in total 11 correlations to stress and ECM genes. These results suggest that a network of 23 stress-related and ECM-related genes is important in the risk for weight regain.

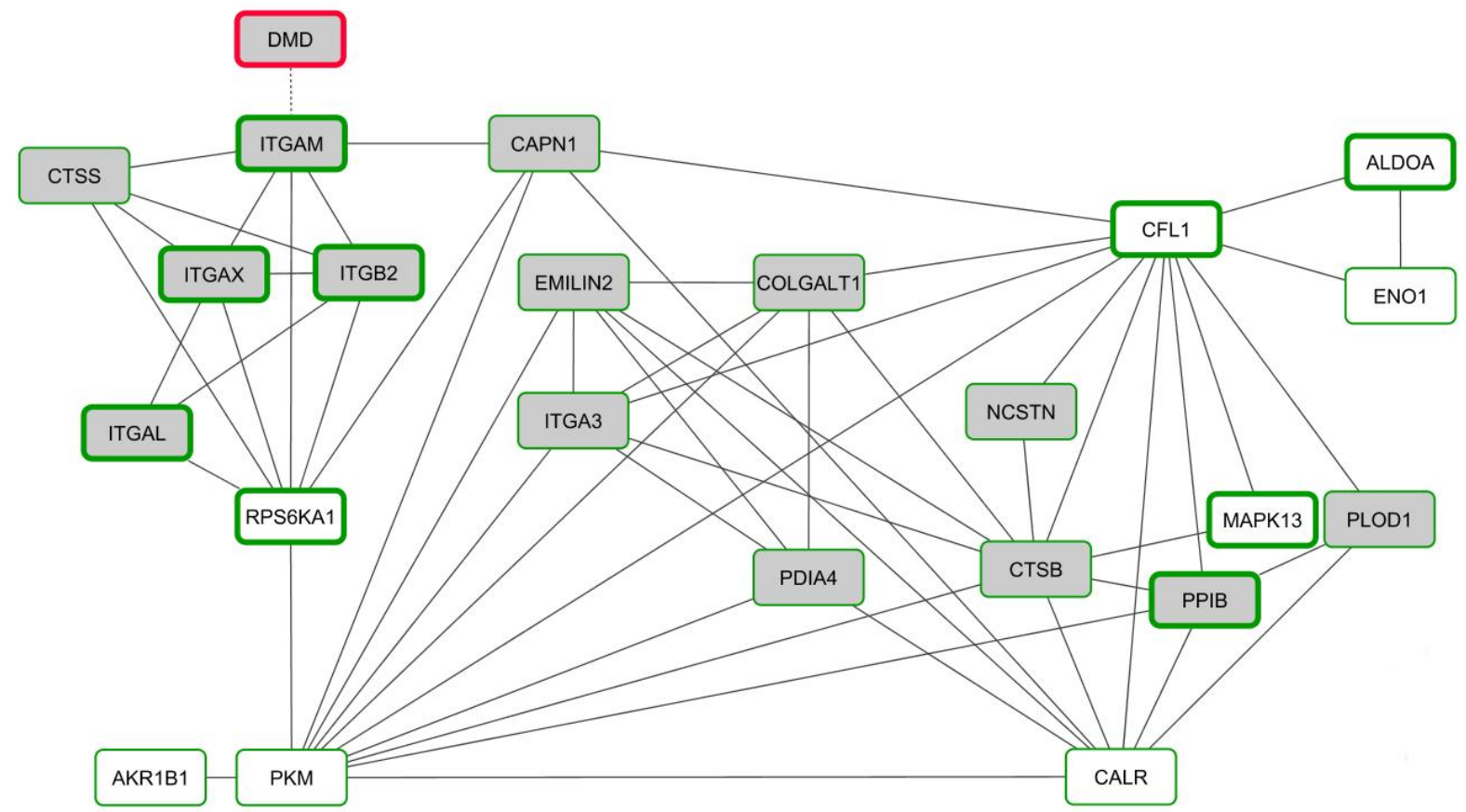

Figure 2: Correlation network between stress-related and ECM-related genes, which strongly correlate with weight regain percentage $(\mathrm{P} \leq 0.05)$, during the weight stabilization phase. Correlations were made using the Pearson correlation coefficient. Only genes that strongly correlate with each other $(|r| \geq 0.800 ; \mathrm{P} \leq 0.001)$ and with the weight regain percentage $(P \leq 0.01)$ are depicted in this figure. White boxes, stress-related genes; grey boxes, ECM-related genes; solid line, positive correlation with other gene; dashed line, negative correlation with other gene; green outline, positive correlation with the weight regain percentage; red outline, negative correlation with the weight regain percentage. Thick outline, strong correlation with weight regain percentage $(P \leq 0.01)$.

\section{Stress- and ECM-related genes interaction influences risk for weight regain}

We determined possible interactions between stress- and ECM-related genes and their influence on the WR\%. In total, 141 significant interactions were found during WL, WS and DI that had an effect on the weight regain percentage (Supplement Table 3). The number of interactions was rather 
similar between all phases: 60 during WL, 47 during WS and 34 during DI. However, the genes with multiple interactions with other genes were different for each phase (Table 3). During WL, most interactions were observed for the stress genes GATA binding protein 6 (GATA6), heat shock transcription factor 2 (HSF2) and the ECM gene EGF Like Fibronectin Type III and Laminin G Domains (EGFLAM). During WS, the stress genes activating transcription factor 4 (ATF4), ERBB receptor feedback inhibitor 1 (ERRFI1; 5), and the ECM gene collagen type XXI alpha 1 chain (COL21A1) have most interactions. During DI, most interactions were observed for the stress genes complement 5 (C5) and chloride intracellular channel 1 (CLIC1).

Table 3: The number of interactions between stress-and ECM-related genes during WL, WS and DI.

\begin{tabular}{|c|c|c|c|c|}
\hline Stress genes & $\begin{array}{l}\text { Connections to } \\
\text { ECM genes }\end{array}$ & ECM genes & $\begin{array}{l}\text { Connections to } \\
\text { stress genes }\end{array}$ & Period \\
\hline GATA6 & 7 & EGFLAM & 5 & WL \\
\hline HSF2 & 7 & ADAM11 & 4 & WL \\
\hline HSP90AB4P & 4 & CSGALNACT1 & 3 & WL \\
\hline PHB & 4 & COL1A1 & 3 & WL \\
\hline MAPK11 & 4 & ITGA11 & 3 & WL \\
\hline DDIT3 & 3 & ADAMTS4 & 3 & WL \\
\hline GSTP1 & 2 & NPNT & 3 & WL \\
\hline PGK1 & 2 & KAZALD1 & 2 & WL \\
\hline TAOK2 & 2 & ADAMTS1 & 2 & WL \\
\hline ARHGDIA & 2 & MADCAM1 & 2 & WL \\
\hline ENO1 & 2 & CTGF & 2 & WL \\
\hline LMNA & 2 & MFI2 & 2 & WL \\
\hline BCLAF1 & 2 & COL6A3 & 2 & WL \\
\hline LGALS1 & 2 & ECM1 & 2 & WL \\
\hline HSPD1 & 2 & & & WL \\
\hline KLF2 & 2 & & & WL \\
\hline ATF4 & 12 & COL21A1 & 5 & WS \\
\hline ERRFI1 & 5 & MPZL3 & 3 & WS \\
\hline TPI1 & 4 & VWF & 2 & WS \\
\hline SQSTM1 & 3 & CHI3L1 & 2 & WS \\
\hline TUBB & 2 & NOTCH1 & 2 & WS \\
\hline EGFR & 2 & GFOD2 & 2 & WS \\
\hline STK39 & 2 & & & WS \\
\hline HSP90AB4P & 2 & & & WS \\
\hline $\mathrm{C} 5$ & 11 & ITGAD & 2 & $\mathrm{DI}$ \\
\hline CLIC1 & 5 & CILP & 2 & DI \\
\hline RPS6KA3 & 4 & TIMP4 & 2 & DI \\
\hline TUBB & 2 & CD47 & 2 & DI \\
\hline PKM & 2 & ELN & 2 & DI \\
\hline
\end{tabular}

The amount of strong interactions ( $\mathrm{P} \leq 1.00 \mathrm{E}-04)$ between stress and $\mathrm{ECM}$ genes. $\mathrm{ECM}$, extracellular matrix; WL, weight loss; WS, weight stable; DI, dietary intervention.

There were 15 strong interactions (interaction $\mathrm{P} \leq 1.00 \mathrm{E}-04$ ) of which most were during the WS period (Table 4). We observed some models with a large effect on WR\% (model $r^{2} \geq 0.5$ and $P \leq 0.001$ ). The interaction between the gene for epidermal growth factor receptor (EGFR) and the gene for actinin alpha 1 (ACTN1) during the WS phase had the biggest effect $\left(r^{2}=0.81\right)$ on the WR\% (Table 4). Previously, we already observed the strong correlation between ECM gene ACTN1 and the WR\% (11) but the current analyses showed that EGFR stratifies this correlation. Specifically, the positive correlation between ACTN1 and the WR\% was reinforced when there was an increase of EGFR during the WS phase (Figure 3a). Furthermore, we observed that during the WS phase both ATF4 and ERRFI1 had multiple interactions with ECM genes which all have a large effect on the WR\% (Table 4). One of these was the negative correlation between ERRFI1 and the WR\% was reinforced when there was a decrease of ITGB4 during the WS phase (Figure $3 \mathrm{~b}$ ). 
Table 4: Strongest interactions between stress- and ECM-related genes with the biggest effect on the weight regain percentage.

\begin{tabular}{|c|c|c|c|c|c|}
\hline Stress genes & ECM genes & $\begin{array}{l}\text { Interaction } \\
\text { P-value }\end{array}$ & Model $r^{2}$ & Model P-value & Period \\
\hline ARHGDIA & ADAM11 & $7.88 \mathrm{E}-05$ & 0.593 & $8.66 \mathrm{E}-04$ & $\mathrm{WL}$ \\
\hline GSTP1 & NPNT & $9.80 \mathrm{E}-05$ & 0.665 & $1.55 \mathrm{E}-04$ & WL \\
\hline PHB & ADAMTS4 & 2.74E-05 & 0.688 & $8.42 \mathrm{E}-05$ & WL \\
\hline BCLAF1 & ADAM11 & $7.68 \mathrm{E}-05$ & 0.595 & $8.26 \mathrm{E}-04$ & WL \\
\hline ATF4 & COL15A1 & $3.70 \mathrm{E}-05$ & 0.602 & $4.60 \mathrm{E}-04$ & WS \\
\hline ATF4 & HSPG2 & $4.43 E-05$ & 0.598 & 4.99E-04 & WS \\
\hline ATF4 & HSD17B12 & $2.81 \mathrm{E}-05$ & 0.614 & $3.44 \mathrm{E}-04$ & WS \\
\hline ATF4 & COL21A1 & $9.11 \mathrm{E}-05$ & 0.616 & $3.29 E-04$ & WS \\
\hline EGFR & ACTN1 & $7.05 E-06$ & 0.811 & 4.38E-07 & WS \\
\hline ERRFI1 & CST3 & $1.51 \mathrm{E}-05$ & 0.654 & $1.25 \mathrm{E}-04$ & WS \\
\hline ERRFI1 & ITGB4 & $3.93 \mathrm{E}-05$ & 0.621 & $2.92 \mathrm{E}-04$ & WS \\
\hline ERRFI1 & LAMA3 & 4.19E-05 & 0.614 & 3.46E-04 & WS \\
\hline $\mathrm{C} 5$ & CD47 & $3.07 \mathrm{E}-06$ & 0.711 & 1.29E-05 & $\mathrm{DI}$ \\
\hline $\mathrm{C} 5$ & ERO1LB & 4.75E-05 & 0.630 & 1.46E-04 & DI \\
\hline RPS6KA3 & COL5A3 & 3.23E-05 & 0.591 & 3.85E-04 & DI \\
\hline
\end{tabular}

Strong interactions ( $\mathrm{P} \leq 1.00 \mathrm{E}-04)$ between stress- and ECM-related genes. $r^{2}(\geq 0.500)$ and Model P-value $(\leq 0.001)$ depict the effect of the interaction on the weight regain percentage. ECM, extracellular matrix; WL, weight loss; WS, weight stable; DI, dietary intervention.
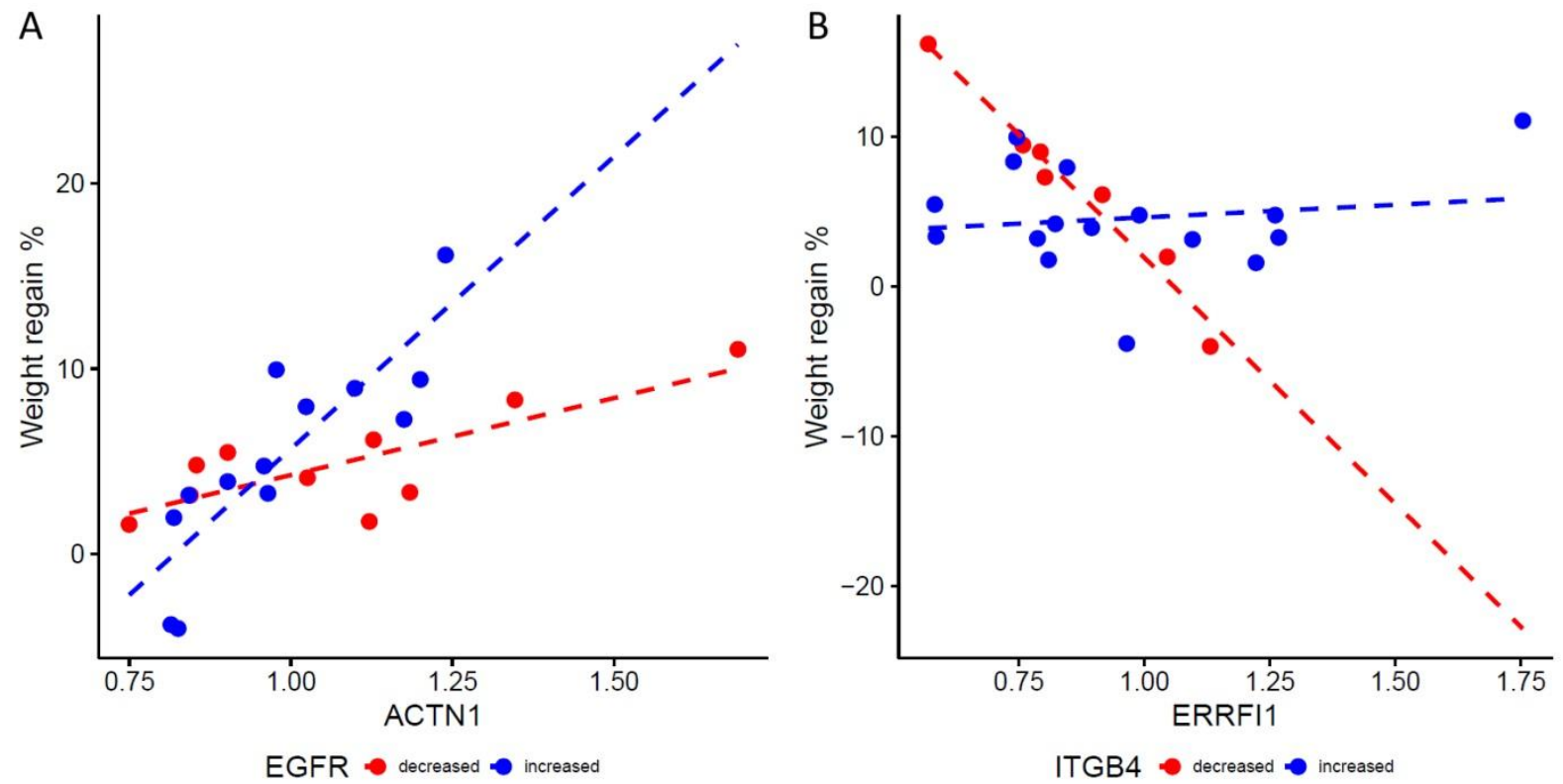

Figure 3: Correlation plot between the WR\% and interaction of the ECM-related gene ACTN1 with the stress-related gene EGFR (A) and the ECM-related gene ITGB4 with the stress-related gene ERRFI1 (B). The lines represent the correlations between changes in WR\% and gene expression of ACTN1 or ERRFI1 at decreasing (red line) or increasing (blue line) gene expression changes of EGFR or ITGB4.

\section{Discussion}

The present study focussed on determining the relation between stress- and ECM-related genes and their combined effect on the weight regain percentage. For this, we selected the stress and ECM genes that correlation $(P \leq 0.05)$ with the WR\%. Correlation analyses between stress and ECM genes revealed that there were 286 strong correlations ( $|r| \geq 0.600 ; \mathrm{P} \leq 0.001)$. Most of these correlations were found in the WS phase and the strongest correlations $(|r| \geq 0.800 ; \mathrm{P} \leq 0.001)$ formed a clear network consisting of 8 stress-related genes and 15 ECM-related genes. Interaction analyses revealed 
that there are many interactions between stress and ECM genes. Fifteen of these interactions have a strong effect $\left(r^{2} \geq 0.5 ; \mathrm{P} \leq 0.001\right)$ indicating that the correlation between expression change of a gene from one category (stress or ECM) and WR\% is highly influenced by the expression change of one or more genes from the other category. The interaction which is most influencing the correlation to the WR\% is that between the stress gene EGFR and the ECM gene ACTN1 in the WS phase.

We have previously reported about the importance of stress genes and proteins in the risk of weight regain. Levels of stress proteins in the adipose tissue of participants regaining weight after weight loss were higher compared to participants maintaining their lost weight (10). Also, we reported that stress genes involved in actin filament dynamics, glucose handling and nutrient sensing are related to weight regain (11). The link between ECM-genes and weight regain has also been established. Genetic variation of ECM genes is associated with an increased risk for weight regain (19). Also, there is a strong relation between the WR\% and ECM genes involved in inflammatory processes and ECM remodelling (12). These studies clearly indicate the involvement of stress-related and ECM-related genes in the risk of weight regain, however, possible interaction was not investigated.

Correlation analyses revealed a co-expression network from 23 stress- and ECM-related genes, which all correlated to the WR\%, during the WS phase. This network seems to consist of a smaller and larger cluster of genes. The smaller cluster consists of 4 leukocyte specific integrins, cathepsin $\mathrm{S}$ (CTSS), RPS6KA1 and DMD, whereas calpain 1 (CAPN1) and PKM form the connection with the larger subcluster. As described previously, the 4 integrins found in the analyses point to the importance of the leukocytes in the risk for weight regain (12). Now, we found that those genes are co-expressed with RPS6KA1. This gene, also known as RSK1, has been identified as a key factor in the regulation of IKB $\beta$ and NF-KB (20), factors involved in regulating the inflammatory response $(21,22)$. CAPN1 and $P K M$, which connect the small subcluster with the larger, are also associated with inflammatory processes, although not specifically to adipose tissue inflammation. In mice, a dramatic reduction of the protein levels and activity of CAPN1 and CAPN2 resulted in the inhibition of inflammatory responses (23). PKM expression increased after treatment of cells with lipopolysaccharide (LPS), a substance used to induce inflammation in vitro, through activation of NF-KB signalling pathway (24).

The larger cluster of genes functions in multiple biological processes. An involvement in inflammatory processes is apparent for some genes, such as elastin microfibril interface 2 (EMILIN2), protein disulfide isomerase family A member 4 (PDIA4), calreticulin (CALR) and cathepsin B (CTSB). Besides a role in the inflammatory process, cathepsins are involved in the remodelling of ECM proteins (25). The cysteine proteases CTSB and CTSL can be secreted outside the cell and digest the ECM (26). In fact, multiple genes in the correlation network are involved in ECM remodelling or cytoskeleton reorganisation. Procollagen-lysine,2-oxoglutarate 5-dioxygenase 1 (PLOD1) and collagen beta(1-0)galactosyltransferase 1 (COLGALT1) are involved in modification of proteins including collagens $(27,28)$. The actin binding protein CFL1 is essential for cytoskeleton remodelling by regulating actin dynamics and plasticity (29). CFL1 can bind to filamentous (F)-actin and is able to depolymerise the filaments allowing dynamic changes of the actin cytoskeleton (13). Cofilin binding to actin can increase the lipid accumulation in the adipose tissue, because CFL controls the mechanical tension of adipocytes $(30,31)$. Also, aldolase A, a glycolytic enzyme and a component of the cytoskeleton, plays an important function in actin cytoskeleton organization $(32,33)$. ALDOA binds to actin filaments, where it may serve in anchoring and translocation of the GLUT4-containing 
vesicles $(2,34)$. These findings suggest that there is increased glucose uptake shortly after returning to energy balance and that this is related to the risk for weight regain. This is supported by the correlation between gene expression change of ENO1and PKM, other enzymes of the glycolysis, and the WR\% during the WS phase. Altogether, our results suggest that the expression patterns of certain leukocyte integrin genes shortly after weight loss by calorie restriction lead to a higher risk for weight regain which appears linked to ECM remodelling, actin cytoskeleton organization, and glucose handling by the adipose tissue.

Interaction analyses revealed 141 significant interactions between stress and ECM genes with an effect on the WR\%. The genes with most interactions with other genes are the stress genes ATF4 during the WS phase and C5 during the DI. Interestingly, 4 of the 12 interactions of ATF4 during the WS phase explained a large part of the WR\% which points to importance of ATF4 in the risk of weight regain. ATF4 has been linked to weight changes previously. ATF4 deficiency is shown to stimulate adipose tissue lipolysis, contributing to the increased energy expenditure in ATF4 knockout mice (35). These mice have a reduced fat mass $(35,36)$. This protects against the development of glucose intolerance and insulin resistance in response to a high fat meal $(37,38)$. Yet, to our knowledge this is the first study reporting about the involvement of ATF4 in weight regain after weight loss. Interestingly, similarly to ATF4 also ERRFI1 has multiple strong interactions during the WS phase that explain a large portion of the WR\%, but the interaction between EGFR and ACTN1 during WS has the strongest effect on the WR\%.

EGFR, also known as ErbB1, plays a key role in adipogenesis and lower levels of ErbB1 can lead to adipose tissue dysfunction (39). EGFR is a transmembrane glycoprotein which is a receptor for members of the epidermal growth factor family. Binding of a ligand to the receptor results in dimerization and activation by autophosphorylation, and downstream activation of various signalling pathways (40). ACTN1 is one of the targets, which can be phosphorylated via activated EGFR. ACTN1 is an actin-binding protein that is found in non-muscle cells along microfilament bundles and adherens-type junctions, where it is involved in binding actin to the membrane. Phosphorylation of alpha-actinin leads to a reduced binding to actin filaments (41). Our finding that a positive correlation between WR\% and ACTN1 during WS is empowered by an increase of the EGFR expression suggests that increased ACTN1 formation is associated with increased ACTN1 phosphorylation in relation to weight regain. Interestingly, among the 15 strong interactions are three involving ERRFI1. ERRFI1, also known as MIG-6, is an inhibitor of EFGR by mutual phosphorylation (42). One of the genes that interact with ERRFI1 is ITGB4. From the correlation plot (Figure 3b) it can be seen that only when the fold change of the expression of ITGB4 decreases, there is an obvious negative correlation between ERRFI1 and WR\%. ITGB4 is able to interact with EGFR in a ligand-independent manner and this complex can trigger focal adhesion kinase (FAK) to activate the AKT signalling pathway (43). FAK is known to be able to phosphorylate alpha-actinin (41). Together these observations indicate that changes in the epidermal growth factor signalling during WS influence the risk of weight regain, which may be mediated by ACTN1 phosphorylation and modified by expression of ITGB4, CST3 and LAMA3 (Figure 4). 


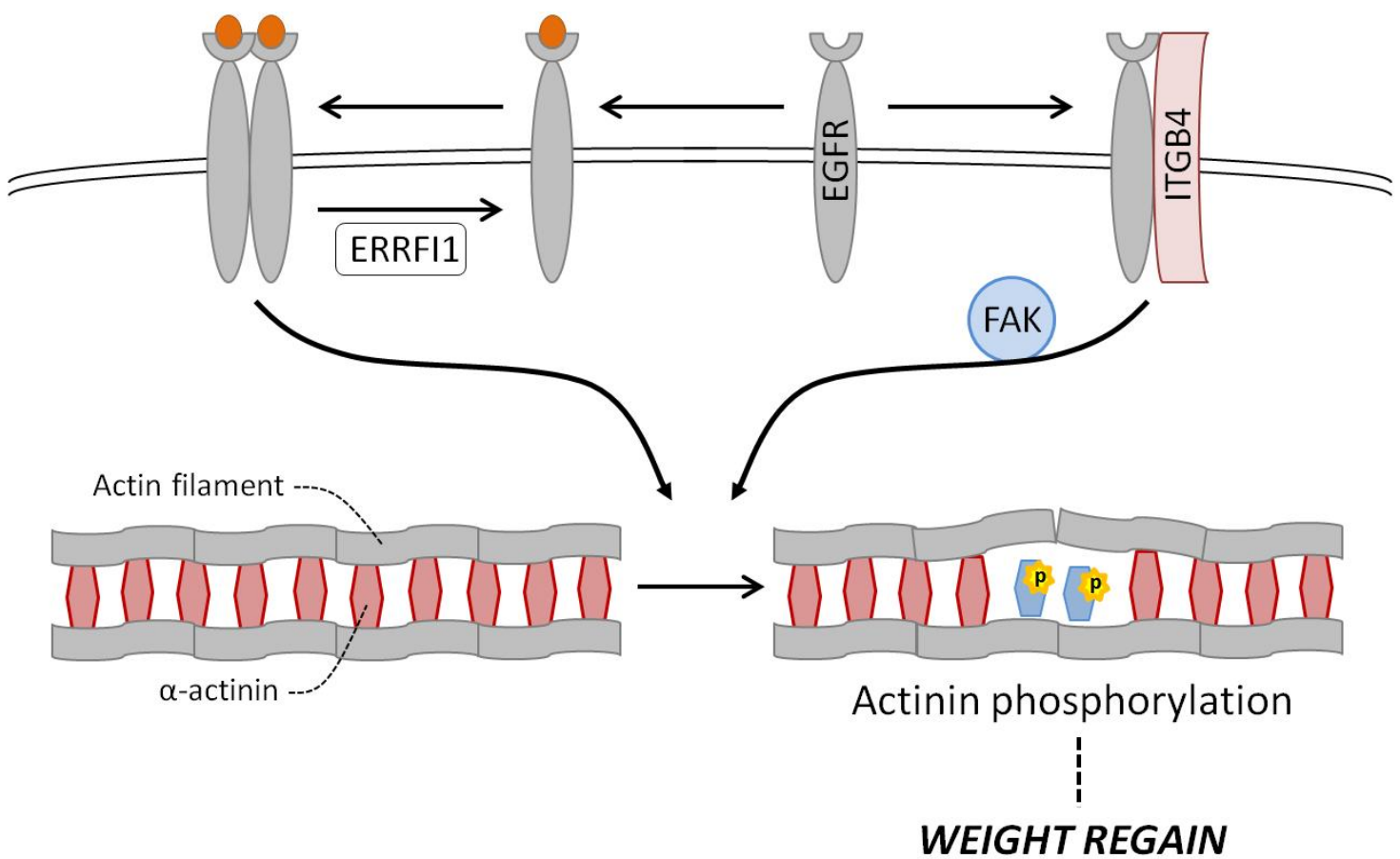

Figure 4: Schematic overview showing that the modification of epidermal growth factor receptor (EGFR) by integrin subunit beta 4 (ITGB4) leads to the phosphorylation of $\alpha$-actinin by focal adhesion kinase (FAK), which might have an effect on the risk for weight regain.

It should be noted that the present study was done in a relative small cohort. A large validation cohort is therefore preferred to confirm our work. Furthermore, although we have used stringent statistical criteria to try to remove possible false positive results, the analyses were based on univariate analysis on two big gene sets which might create an overfitting problem. Nevertheless, we could generate leads to interesting genes and gene interactions for weight regain, which can now be further explored. We should point out that the results are from adipose tissue biopsies containing both adipocytes as well as stromal vascular cells. Therefore, further experiments are preferably performed with purified adipose tissue cell fractions.

In conclusion, a co-expression network of stress- and ECM-related genes correlating with weight regain percentage could be constructed during the WS phase. The biological processes linked to this network were mainly focussed on leukocyte-activity, ECM remodelling, actin cytoskeleton organisation and glucose handling. Interaction analysis between stress- and ECM-related genes revealed several gene combinations that were highly related to the WR\%. In particular, the epidermal growth factor signaling pathway was identified as strongly influencing the risk of weight regain, possibly through interaction with ACTN1, a component of stress fibers, and with ITGB4, CTS3 and LAMA3. 


\section{References}

1. Wang P, Bouwman FG, Mariman EC. Generally detected proteins in comparative proteomics--a matter of cellular stress response? Proteomics, 2009. 9(11):2955-66.

2. Bouwman FG, Claessens M, van Baak MA, Noben JP, Wang P, Saris WH, Mariman EC. The physiologic effects of caloric restriction are reflected in the in vivo adipocyte-enriched proteome of overweight/obese subjects. J Proteome Res, 2009. 8(12):5532-40.

3. Ng M, Fleming $\mathrm{T}$, Robinson $\mathrm{M}$, Thomson $\mathrm{B}$, Graetz $\mathrm{N}$, Margono $\mathrm{C}$ et al. Global, regional, and national prevalence of overweight and obesity in children and adults during 1980-2013: a systematic analysis for the Global Burden of Disease Study 2013. Lancet, 2014. 384(9945):766-81.

4. Van Gaal LF, Wauters MA, De Leeuw IH. The beneficial effects of modest weight loss on cardiovascular risk factors. Int J Obes Relat Metab Disord, 1997. 21 Suppl 1:S5-9.

5. Weinstock RS, Dai H, Wadden TA. Diet and exercise in the treatment of obesity: effects of 3 interventions on insulin resistance. Arch Intern Med, 1998. 158(22):2477-83.

6. Barte JC, ter Bogt NC, Bogers RP, Teixeira PJ, Blissmer B, Mori TA, Bemelmans WJ. Maintenance of weight loss after lifestyle interventions for overweight and obesity, a systematic review. Obes Rev, 2010. 11(12):899-906.

7. Wu T, Gao X, Chen M, van Dam RM. Long-term effectiveness of diet-plus-exercise interventions vs. diet-only interventions for weight loss: a meta-analysis. Obes Rev, 2009. 10(3):313-23.

8. Blomain ES, Dirhan DA, Valentino MA, Kim GW, Waldman SA. Mechanisms of Weight Regain following Weight Loss. ISRN obesity, 2013. 2013:210524.

9. Delahanty LM, Pan Q, Jablonski KA, Aroda VR, Watson KE, Bray GA et al. Effects of weight loss, weight cycling, and weight loss maintenance on diabetes incidence and change in cardiometabolic traits in the Diabetes Prevention Program. Diabetes Care, 2014. 37(10):2738-45.

10. Roumans NJ, Camps SG, Renes J, Bouwman FG, Westerterp KR, Mariman EC. Weight loss-induced stress in subcutaneous adipose tissue is related to weight regain. Br J Nutr, 2016. 115(5):913-20.

11. Roumans NJ, Vink RG, Bouwman FG, Fazelzadeh P, van Baak MA, Mariman EC. Weight loss-induced cellular stress in subcutaneous adipose tissue and the risk for weight regain in overweight and obese adults. International journal of obesity, 2016.

12. Roumans NJ, Vink RG, Fazelzadeh P, van Baak MA, Mariman EC. A role for leukocyte integrins and extracellular matrix remodeling of adipose tissue in the risk of weight regain after weight loss. Am J Clin Nutr, 2017.

13. Shawlot W, Deng JM, Fohn LE, Behringer RR. Restricted beta-galactosidase expression of a hygromycin-lacZ gene targeted to the beta-actin locus and embryonic lethality of beta-actin mutant mice. Transgenic research, 1998. 7(2):95-103.

14. Shannon P, Markiel A, Ozier O, Baliga NS, Wang JT, Ramage D, Amin N, Schwikowski B, Ideker T. Cytoscape: a software environment for integrated models of biomolecular interaction networks. Genome Res, 2003. 13(11):2498-504.

15. Vink RG, Roumans NJ, Arkenbosch LA, Mariman EC, van Baak MA. The effect of rate of weight loss on long-term weight regain in adults with overweight and obesity. Obesity (Silver Spring), 2016. 24(2):321-7.

16. Smyth GK. Limma: linear models for microarray data. Edtion ed. Bioinformatics and computational biology solutions using R and Bioconductor: Springer, 2005:397-420.

17. Benjamini Y, Hochberg Y. Controlling the False Discovery Rate - a Practical and Powerful Approach to Multiple Testing. J Roy Stat Soc B Met, 1995. 57(1):289-300.

18. Karnovsky A, Weymouth T, Hull T, Tarcea VG, Scardoni G, Laudanna C et al. Metscape 2 bioinformatics tool for the analysis and visualization of metabolomics and gene expression data. Bioinformatics, 2012. 28(3):373-80.

19. Roumans NJ, Vink RG, Gielen M, Zeegers MP, Holst C, Wang P et al. Variation in extracellular matrix genes is associated with weight regain after weight loss in a sex-specific manner. Genes Nutr, 2015. 10(6):56.

20. Xu S, Bayat H, Hou X, Jiang B. Ribosomal S6 kinase-1 modulates interleukin-1beta-induced persistent activation of NF-kappaB through phosphorylation of IkappaBbeta. American journal of physiology Cell physiology, 2006. 291(6):C1336-45.

21. Baeuerle PA, Baltimore D. NF-kappa B: ten years after. Cell, 1996. 87(1):13-20.

22. Baldwin AS, Jr. The NF-kappa B and I kappa B proteins: new discoveries and insights. Annual review of immunology, 1996. 14:649-83. 
23. Ma J, Wei M, Wang Q, Li J, Wang H, Liu W et al. Deficiency of Capn4 gene inhibits nuclear factorkappaB (NF-kappaB) protein signaling/inflammation and reduces remodeling after myocardial infarction. The Journal of biological chemistry, 2012. 287(33):27480-9.

24. Yang P, Li Z, Li H, Lu Y, Wu H, Li Z. Pyruvate kinase M2 accelerates pro-inflammatory cytokine secretion and cell proliferation induced by lipopolysaccharide in colorectal cancer. Cellular signalling, 2015. 27(7):1525-32.

25. Fiebiger E, Maehr R, Villadangos J, Weber E, Erickson A, Bikoff E, Ploegh HL, Lennon-Dumenil AM. Invariant chain controls the activity of extracellular cathepsin L. The Journal of experimental medicine, 2002. 196(9):1263-9.

26. Lu P, Takai K, Weaver VM, Werb Z. Extracellular matrix degradation and remodeling in development and disease. Cold Spring Harbor perspectives in biology, 2011. 3(12).

27. Eyre D, Shao P, Weis MA, Steinmann B. The kyphoscoliotic type of Ehlers-Danlos syndrome (type VI): differential effects on the hydroxylation of lysine in collagens I and II revealed by analysis of crosslinked telopeptides from urine. Molecular genetics and metabolism, 2002. 76(3):211-6.

28. Liefhebber JM, Punt S, Spaan WJ, van Leeuwen HC. The human collagen beta(1O)galactosyltransferase, GLT25D1, is a soluble endoplasmic reticulum localized protein. BMC cell biology, 2010. 11:33.

29. Theriot JA. Accelerating on a treadmill: ADF/cofilin promotes rapid actin filament turnover in the dynamic cytoskeleton. The Journal of cell biology, 1997. 136(6):1165-8.

30. Choi KC, Roh SG, Hishikawa D, Miyahara H, Kuno M, Tsuzuki H et al. Differential expression of the nonmuscle-type cofilin gene between subcutaneous and visceral adipose tissue. Bioscience, biotechnology, and biochemistry, 2003. 67(10):2262-5.

31. Maciver SK, Hussey PJ. The ADF/cofilin family: actin-remodeling proteins. Genome biology, 2002. 3(5):reviews3007.

32. Mamczur P, Gamian A, Kolodziej J, Dziegiel P, Rakus D. Nuclear localization of aldolase A correlates with cell proliferation. Biochimica et biophysica acta, 2013. 1833(12):2812-22.

33. Merkulova M, Hurtado-Lorenzo A, Hosokawa H, Zhuang Z, Brown D, Ausiello DA, Marshansky V. Aldolase directly interacts with ARNO and modulates cell morphology and acidic vesicle distribution. American journal of physiology Cell physiology, 2011. 300(6):C1442-55.

34. Kao AW, Noda Y, Johnson JH, Pessin JE, Saltiel AR. Aldolase mediates the association of F-actin with the insulin-responsive glucose transporter GLUT4. The Journal of biological chemistry, 1999. 274(25):17742-7.

35. Wang C, Huang Z, Du Y, Cheng Y, Chen S, Guo F. ATF4 regulates lipid metabolism and thermogenesis. Cell research, 2010. 20(2):174-84.

36. Yoshizawa T, Hinoi E, Jung DY, Kajimura D, Ferron M, Seo J, Graff JM, Kim JK, Karsenty G. The transcription factor ATF4 regulates glucose metabolism in mice through its expression in osteoblasts. The Journal of clinical investigation, 2009. 119(9):2807-17.

37. Li H, Meng Q, Xiao F, Chen S, Du Y, Yu J, Wang C, Guo F. ATF4 deficiency protects mice from highcarbohydrate-diet-induced liver steatosis. The Biochemical journal, 2011. 438(2):283-9.

38. Seo J, Fortuno ES, 3rd, Suh JM, Stenesen D, Tang W, Parks EJ, Adams CM, Townes T, Graff JM. Atf4 regulates obesity, glucose homeostasis, and energy expenditure. Diabetes, 2009. 58(11):2565-73.

39. Rogers C, Moukdar F, McGee MA, Davis B, Buehrer BM, Daniel KW, Collins S, Barakat H, Robidoux J. EGF receptor (ERBB1) abundance in adipose tissue is reduced in insulin-resistant and type 2 diabetic women. The Journal of clinical endocrinology and metabolism, 2012. 97(3):E329-40.

40. Yarden Y, Schlessinger J. Self-phosphorylation of epidermal growth factor receptor: evidence for a model of intermolecular allosteric activation. Biochemistry, 1987. 26(5):1434-42.

41. Izaguirre G, Aguirre L, Hu YP, Lee HY, Schlaepfer DD, Aneskievich BJ, Haimovich B. The cytoskeletal/non-muscle isoform of alpha-actinin is phosphorylated on its actin-binding domain by the focal adhesion kinase. The Journal of biological chemistry, 2001. 276(31):28676-85.

42. Park E, Kim N, Ficarro SB, Zhang Y, Lee BI, Cho A et al. Structure and mechanism of activity-based inhibition of the EGF receptor by Mig6. Nature structural \& molecular biology, 2015. 22(9):703-11.

43. Leng C, Zhang ZG, Chen WX, Luo HP, Song J, Dong W, Zhu XR, Chen XP, Liang HF, Zhang BX. An integrin beta4-EGFR unit promotes hepatocellular carcinoma lung metastases by enhancing anchorage independence through activation of FAK-AKT pathway. Cancer letters, 2016. 376(1):188-96. 
Supplementary Figures and Tables 

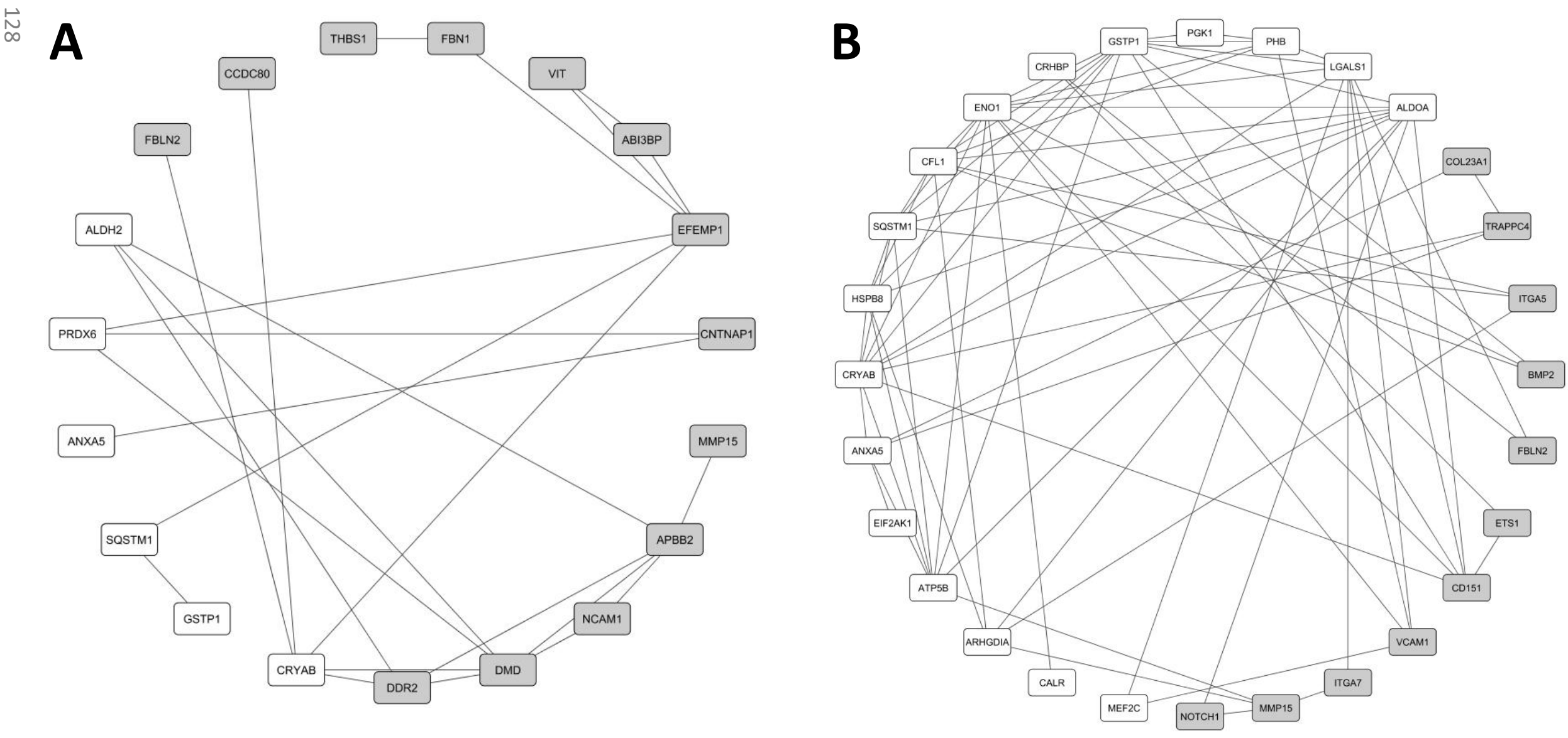

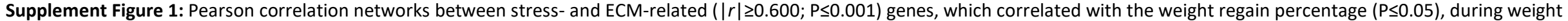
loss (A) and dietary intervention (B). White boxes, stress-related genes; grey boxes, ECM-related genes. 
Supplement Table 1: List of the 107 stress-related genes and the 277 extracellular matrix related genes used for the analysis.

\begin{tabular}{|c|c|c|c|}
\hline Gene ID & Gene name & Gene \# & Group \\
\hline ACTB & actin, beta & 60 & Stress \\
\hline ACTG1 & actin gamma 1 & 71 & Stress \\
\hline AHSA2 & AHA1, activator of heat shock 90kDa protein ATPase homolog 2 (yeast) & 130872 & Stress \\
\hline AKR1B1 & aldo-keto reductase family 1 , member B1 (aldose reductase) & 231 & Stress \\
\hline $\mathrm{ALDH} 2$ & aldehyde dehydrogenase 2 family (mitochondrial) & 217 & Stress \\
\hline ALDOA & aldolase $A$, fructose-bisphosphate & 226 & Stress \\
\hline ANXA2 & annexin A2 & 302 & Stress \\
\hline ANXA5 & annexin A5 & 308 & Stress \\
\hline AQP1 & aquaporin 1 (Colton blood group) & 358 & Stress \\
\hline ARHGDIA & Rho GDP dissociation inhibitor (GDI) alpha & 396 & Stress \\
\hline ATF3 & activating transcription factor 3 & 467 & Stress \\
\hline ATF4 & activating transcription factor 4 & 468 & Stress \\
\hline ATP2B4 & ATPase, $\mathrm{Ca}++$ transporting, plasma membrane 4 & 493 & Stress \\
\hline ATP5B & ATP synthase, $\mathrm{H}+$ transporting, mitochondrial F1 complex, beta polypeptide & 506 & Stress \\
\hline BCLAF1 & BCL2-associated transcription factor 1 & 9774 & Stress \\
\hline C5 & complement component 5 & 727 & Stress \\
\hline CALR & Calreticulin & 811 & Stress \\
\hline CFL1 & cofilin 1 (non-muscle) & 1072 & Stress \\
\hline CHORDC1 & cysteine and histidine-rich domain (CHORD) containing 1 & 26973 & Stress \\
\hline CLIC1 & chloride intracellular channel 1 & 1192 & Stress \\
\hline CRHBP & corticotropin releasing hormone binding protein & 1393 & Stress \\
\hline CRYAB & crystallin, alpha B & 1410 & Stress \\
\hline CTSD & cathepsin D & 1509 & Stress \\
\hline DDIT3 & DNA-damage-inducible transcript 3 & 1649 & Stress \\
\hline DNAJC3 & DnaJ (Hsp40) homolog, subfamily C, member 3 & 5611 & Stress \\
\hline DUSP10 & dual specificity phosphatase 10 & 11221 & Stress \\
\hline EDEM1 & ER degradation enhancer, mannosidase alpha-like 1 & 9695 & Stress \\
\hline EGFR & epidermal growth factor receptor & 1956 & Stress \\
\hline EIF2AK1 & eukaryotic translation initiation factor 2-alpha kinase 1 & 27102 & Stress \\
\hline EIF2B3 & eukaryotic translation initiation factor 2B, subunit 3 gamma, $58 \mathrm{kDa}$ & 8891 & Stress \\
\hline EIF2S1 & eukaryotic translation initiation factor 2 , subunit 1 alpha, $35 \mathrm{kDa}$ & 1965 & Stress \\
\hline EIF5A & eukaryotic translation initiation factor $5 \mathrm{~A}$ & 1984 & Stress \\
\hline ENO1 & enolase 1, (alpha) & 2023 & Stress \\
\hline ERRFI1 & ERBB receptor feedback inhibitor 1 & 54206 & Stress \\
\hline GADD45B & growth arrest and DNA-damage-inducible, beta & 4616 & Stress \\
\hline GADD45G & growth arrest and DNA-damage-inducible, gamma & 10912 & Stress \\
\hline GATA4 & GATA binding protein 4 & 2626 & Stress \\
\hline GATA6 & GATA binding protein 6 & 2627 & Stress \\
\hline GSTP1 & glutathione S-transferase pi 1 & 2950 & Stress \\
\hline HEY2 & hes-related family bHLH transcription factor with YRPW motif 2 & 23493 & Stress \\
\hline HILPDA & hypoxia inducible lipid droplet-associated & 29923 & Stress \\
\hline HSF2 & heat shock transcription factor 2 & 3298 & Stress \\
\hline HSF4 & heat shock transcription factor 4 & 3299 & Stress \\
\hline HSP90AB4P & heat shock protein 90kDa alpha (cytosolic), class B member 4, pseudogene & 664618 & Stress \\
\hline HSP90B2P & heat shock protein 90kDa beta (Grp94), member 2, pseudogene & 7190 & Stress \\
\hline HSPA5 & heat shock 70kDa protein 5 (glucose-regulated protein, 78kDa) & 3309 & Stress \\
\hline HSPA8 & heat shock 70kDa protein 8 & 3312 & Stress \\
\hline HSPB1 & heat shock $27 \mathrm{kDa}$ protein 1 & 3315 & Stress \\
\hline HSPB11 & heat shock protein family B (small), member 11 & 51668 & Stress \\
\hline HSPB6 & heat shock protein, alpha-crystallin-related, B6 & 126393 & Stress \\
\hline HSPB8 & heat shock $22 \mathrm{kDa}$ protein 8 & 26353 & Stress \\
\hline HSPD1 & heat shock 60kDa protein 1 (chaperonin) & 3329 & Stress \\
\hline HYOU1 & hypoxia up-regulated 1 & 10525 & Stress \\
\hline IDO1 & indoleamine 2,3-dioxygenase 1 & 3620 & Stress \\
\hline KLF15 & Kruppel-like factor 15 & 28999 & Stress \\
\hline KLF2 & Kruppel-like factor 2 & 10365 & Stress \\
\hline KRT8 & keratin 8, type II & 3856 & Stress \\
\hline LGALS1 & lectin, galactoside-binding, soluble, 1 & 3956 & Stress \\
\hline LMNA & $\operatorname{lamin} \mathrm{A} / \mathrm{C}$ & 4000 & Stress \\
\hline MAP4K1 & mitogen-activated protein kinase kinase kinase kinase 1 & 11184 & Stress \\
\hline MAPK11 & mitogen-activated protein kinase 11 & 5600 & Stress \\
\hline MAPK13 & mitogen-activated protein kinase 13 & 5603 & Stress \\
\hline MAPK7 & mitogen-activated protein kinase 7 & 5598 & Stress \\
\hline MAPK8 & mitogen-activated protein kinase 8 & 5599 & Stress \\
\hline MAPK9 & mitogen-activated protein kinase 9 & 5601 & Stress \\
\hline MAPKAPK3 & mitogen-activated protein kinase-activated protein kinase 3 & 7867 & Stress \\
\hline MEF2C & myocyte enhancer factor $2 \mathrm{C}$ & 4208 & Stress \\
\hline
\end{tabular}




\begin{tabular}{|c|c|c|c|}
\hline MUC1 & mucin 1 , cell surface associated & 4582 & Stress \\
\hline NDRG4 & NDRG family member 4 & 65009 & Stress \\
\hline NME2 & NME/NM23 nucleoside diphosphate kinase 2 & 4831 & Stress \\
\hline NPM1 & nucleophosmin (nucleolar phosphoprotein B23, numatrin) & 4869 & Stress \\
\hline OMA1 & OMA1 zinc metallopeptidase & 115209 & Stress \\
\hline P4HB & prolyl 4-hydroxylase, beta polypeptide & 5034 & Stress \\
\hline PGK1 & phosphoglycerate kinase 1 & 5230 & Stress \\
\hline PHB & Prohibitin & 5245 & Stress \\
\hline PKM & pyruvate kinase, muscle & 5315 & Stress \\
\hline PPIA & peptidylprolyl isomerase A (cyclophilin A) & 5478 & Stress \\
\hline PRDX6 & peroxiredoxin 6 & 9588 & Stress \\
\hline PRKAA2 & protein kinase, AMP-activated, alpha 2 catalytic subunit & 5563 & Stress \\
\hline PSME1 & proteasome (prosome, macropain) activator subunit 1 (PA28 alpha) & 5720 & Stress \\
\hline PTEN & phosphatase and tensin homolog & 5728 & Stress \\
\hline PTK2B & protein tyrosine kinase 2 beta & 2185 & Stress \\
\hline RGS14 & regulator of G-protein signaling 14 & 10636 & Stress \\
\hline RPS6KA1 & ribosomal protein $\mathrm{S} 6$ kinase, $90 \mathrm{kDa}$, polypeptide 1 & 6195 & Stress \\
\hline RPS6KA3 & ribosomal protein $\mathrm{S} 6$ kinase, $90 \mathrm{kDa}$, polypeptide 3 & 6197 & Stress \\
\hline RTN3 & reticulon 3 & 10313 & Stress \\
\hline SGK1 & serum/glucocorticoid regulated kinase 1 & 6446 & Stress \\
\hline SNN & Stannin & 8303 & Stress \\
\hline SQSTM1 & sequestosome 1 & 8878 & Stress \\
\hline STC2 & stanniocalcin 2 & 8614 & Stress \\
\hline STIP1 & stress-induced phosphoprotein 1 & 10963 & Stress \\
\hline STK39 & serine threonine kinase 39 & 27347 & Stress \\
\hline STMN1 & stathmin 1 & 3925 & Stress \\
\hline TAOK2 & TAO kinase 2 & 9344 & Stress \\
\hline TCAP & titin-cap & 8557 & Stress \\
\hline TENM1 & teneurin transmembrane protein 1 & 10178 & Stress \\
\hline TMEM204 & transmembrane protein 204 & 79652 & Stress \\
\hline TP53I11 & tumor protein p53 inducible protein 11 & 9537 & Stress \\
\hline TP53INP1 & tumor protein p53 inducible nuclear protein 1 & 94241 & Stress \\
\hline TP53TG1 & TP53 target 1 (non-protein coding) & 11257 & Stress \\
\hline TPI1 & triosephosphate isomerase 1 & 7167 & Stress \\
\hline TPM3 & tropomyosin 3 & 7170 & Stress \\
\hline TRAF2 & TNF receptor-associated factor 2 & 7186 & Stress \\
\hline TUBB & tubulin, beta class I & 203068 & Stress \\
\hline UCN2 & urocortin 2 & 90226 & Stress \\
\hline ZAK & sterile alpha motif and leucine zipper containing kinase AZK & 51776 & Stress \\
\hline $\mathrm{A} 2 \mathrm{M}$ & alpha-2-macroglobulin & 2 & ECM \\
\hline ABI3BP & ABI family, member 3 (NESH) binding protein & 25890 & ECM \\
\hline ACTN1 & actinin, alpha 1 & 87 & ECM \\
\hline ADAM10 & ADAM metallopeptidase domain 10 & 102 & ECM \\
\hline ADAM11 & ADAM metallopeptidase domain 11 & 4185 & ECM \\
\hline ADAM12 & ADAM metallopeptidase domain 12 & 8038 & ECM \\
\hline ADAM15 & ADAM metallopeptidase domain 15 & 8751 & ECM \\
\hline ADAM17 & ADAM metallopeptidase domain 17 & 6868 & ECM \\
\hline ADAM19 & ADAM metallopeptidase domain 19 & 8728 & ECM \\
\hline ADAM22 & ADAM metallopeptidase domain 22 & 53616 & ECM \\
\hline ADAM8 & ADAM metallopeptidase domain 8 & 101 & ECM \\
\hline ADAM9 & ADAM metallopeptidase domain 9 & 8754 & ECM \\
\hline ADAMTS1 & ADAM metallopeptidase with thrombospondin type 1 motif, 1 & 9510 & ECM \\
\hline ADAMTS14 & ADAM metallopeptidase with thrombospondin type 1 motif, 14 & 140766 & ECM \\
\hline ADAMTS2 & ADAM metallopeptidase with thrombospondin type 1 motif, 2 & 9509 & ECM \\
\hline ADAMTS4 & ADAM metallopeptidase with thrombospondin type 1 motif, 4 & 9507 & ECM \\
\hline ADAMTS5 & ADAM metallopeptidase with thrombospondin type 1 motif, 5 & 11096 & ECM \\
\hline ADAMTSL4 & ADAMTS-like 4 & 54507 & ECM \\
\hline AGRN & Agrin & 375790 & ECM \\
\hline AGT & angiotensinogen (serpin peptidase inhibitor, clade $A$, member 8) & 183 & ECM \\
\hline APBB2 & amyloid beta (A4) precursor protein-binding, family $B$, member 2 & 323 & ECM \\
\hline AZGP1 & alpha-2-glycoprotein 1, zinc-binding & 563 & ECM \\
\hline $\mathrm{BCL3}$ & B-cell CLL/lymphoma 3 & 602 & ECM \\
\hline BGN & Biglycan & 633 & ECM \\
\hline BMP1 & bone morphogenetic protein 1 & 649 & ECM \\
\hline BMP2 & bone morphogenetic protein 2 & 650 & ECM \\
\hline BMP4 & bone morphogenetic protein 4 & 652 & ECM \\
\hline BSG & basigin (Ok blood group) & 682 & ECM \\
\hline CAPN1 & calpain $1,(\mathrm{mu} / \mathrm{l})$ large subunit & 823 & ECM \\
\hline CASP3 & caspase 3 , apoptosis-related cysteine peptidase & 836 & ECM \\
\hline CCDC80 & coiled-coil domain containing 80 & 151887 & ECM \\
\hline 151 & CD151 molecule (Raph blood grou & 977 & FCM \\
\hline
\end{tabular}




\begin{tabular}{|c|c|c|c|}
\hline CD44 & CD44 molecule (Indian blood group) & 960 & ECM \\
\hline CD47 & CD47 molecule & 961 & ECM \\
\hline CER1 & cerberus 1, DAN family BMP antagonist & 9350 & ECM \\
\hline CHI3L1 & chitinase 3-like 1 (cartilage glycoprotein-39) & 1116 & ECM \\
\hline CIB1 & calcium and integrin binding 1 (calmyrin) & 10519 & ECM \\
\hline CILP & cartilage intermediate layer protein, nucleotide pyrophosphohydrolase & 8483 & ECM \\
\hline CMA1 & chymase 1 , mast cell & 1215 & ECM \\
\hline CNTNAP1 & contactin associated protein 1 & 8506 & ECM \\
\hline COL11A1 & collagen, type $\mathrm{XI}$, alpha 1 & 1301 & ECM \\
\hline COL12A1 & collagen, type XII, alpha 1 & 1303 & ECM \\
\hline COL13A1 & collagen, type XIII, alpha 1 & 1305 & ECM \\
\hline COL14A1 & collagen, type XIV, alpha 1 & 7373 & ECM \\
\hline COL15A1 & collagen, type XV, alpha 1 & 1306 & ECM \\
\hline COL16A1 & collagen, type XVI, alpha 1 & 1307 & ECM \\
\hline COL17A1 & collagen, type XVII, alpha 1 & 1308 & ECM \\
\hline COL18A1 & collagen, type XVIII, alpha 1 & 80781 & ECM \\
\hline COL1A1 & collagen, type I, alpha 1 & 1277 & ECM \\
\hline COL1A2 & collagen, type I, alpha 2 & 1278 & ECM \\
\hline COL20A1 & collagen, type XX, alpha 1 & 57642 & ECM \\
\hline COL21A1 & collagen, type XXI, alpha 1 & 81578 & ECM \\
\hline COL23A1 & collagen, type XXIII, alpha 1 & 91522 & ECM \\
\hline COL25A1 & collagen, type XXV, alpha 1 & 84570 & ECM \\
\hline COL26A1 & collagen, type $X X V I$, alpha 1 & 136227 & ECM \\
\hline COL27A1 & collagen, type XXVII, alpha 1 & 85301 & ECM \\
\hline COL2A1 & collagen, type II, alpha 1 & 1280 & ECM \\
\hline COL3A1 & collagen, type III, alpha 1 & 1281 & ECM \\
\hline COL4A1 & collagen, type IV, alpha 1 & 1282 & ECM \\
\hline COL4A2 & collagen, type IV, alpha 2 & 1284 & ECM \\
\hline COL4A5 & collagen, type IV, alpha 5 & 1287 & ECM \\
\hline COL5A1 & collagen, type $\mathrm{V}$, alpha 1 & 1289 & ECM \\
\hline COL5A2 & collagen, type $V$, alpha 2 & 1290 & ECM \\
\hline COL5A3 & collagen, type $\mathrm{V}$, alpha 3 & 50509 & ECM \\
\hline COL6A1 & collagen, type $\mathrm{VI}$, alpha 1 & 1291 & ECM \\
\hline COL6A2 & collagen, type VI, alpha 2 & 1292 & ECM \\
\hline COL6A3 & collagen, type VI, alpha 3 & 1293 & ECM \\
\hline COL6A6 & collagen, type VI, alpha 6 & 131873 & ECM \\
\hline COL8A1 & collagen, type VIII, alpha 1 & 1295 & ECM \\
\hline COL8A2 & collagen, type VIII, alpha 2 & 1296 & ECM \\
\hline COL9A2 & collagen, type IX, alpha 2 & 1298 & ECM \\
\hline COL9A3 & collagen, type IX, alpha 3 & 1299 & ECM \\
\hline COLGALT1 & collagen beta(1-0)galactosyltransferase 1 & 79709 & ECM \\
\hline COMP & cartilage oligomeric matrix protein & 1311 & ECM \\
\hline CRISPLD2 & cysteine-rich secretory protein LCCL domain containing 2 & 83716 & ECM \\
\hline CRTAP & cartilage associated protein & 10491 & ECM \\
\hline CSGALNACT1 & chondroitin sulfate $\mathrm{N}$-acetylgalactosaminyltransferase 1 & 55790 & ECM \\
\hline CSPG4 & chondroitin sulfate proteoglycan 4 & 1464 & ECM \\
\hline CST3 & cystatin C & 1471 & ECM \\
\hline CTGF & connective tissue growth factor & 1490 & ECM \\
\hline CTRB1 & chymotrypsinogen B1 & 1504 & ECM \\
\hline CTRB2 & chymotrypsinogen B2 & 440387 & ECM \\
\hline CTSB & cathepsin B & 1508 & ECM \\
\hline CTSG & cathepsin G & 1511 & ECM \\
\hline CTSK & cathepsin K & 1513 & ECM \\
\hline CTSL & cathepsin L & 1514 & ECM \\
\hline CTSS & cathepsin S & 1520 & ECM \\
\hline CYP1B1 & cytochrome P450, family 1 , subfamily B, polypeptide 1 & 1545 & ECM \\
\hline CYR61 & cysteine-rich, angiogenic inducer, 61 & 3491 & ECM \\
\hline DAG1 & dystroglycan 1 (dystrophin-associated glycoprotein 1) & 1605 & ECM \\
\hline DCN & Decorin & 1634 & ECM \\
\hline DDR1 & discoidin domain receptor tyrosine kinase 1 & 780 & ECM \\
\hline DDR2 & discoidin domain receptor tyrosine kinase 2 & 4921 & ECM \\
\hline DMD & Dystrophin & 1756 & ECM \\
\hline DPP4 & dipeptidyl-peptidase 4 & 1803 & ECM \\
\hline DPT & Dermatopontin & 1805 & ECM \\
\hline ECM1 & extracellular matrix protein 1 & 1893 & ECM \\
\hline ECM2 & extracellular matrix protein 2 , female organ and adipocyte specific & 1842 & ECM \\
\hline EFEMP1 & EGF containing fibulin-like extracellular matrix protein 1 & 2202 & ECM \\
\hline EFEMP2 & EGF containing fibulin-like extracellular matrix protein 2 & 30008 & ECM \\
\hline EGFL6 & EGF-like-domain, multiple 6 & 25975 & ECM \\
\hline EGFLAM & EGF-like, fibronectin type III and laminin $\mathrm{G}$ domains & 133584 & ECM \\
\hline ELANE & elastase, neutrophil expressed & 1991 & ECM \\
\hline
\end{tabular}




\begin{tabular}{|c|c|c|c|}
\hline ELN & Elastin & 2006 & ECM \\
\hline EMILIN1 & elastin microfibril interfacer 1 & 11117 & ECM \\
\hline EMILIN2 & elastin microfibril interfacer 2 & 84034 & ECM \\
\hline ENG & Endoglin & 2022 & $\mathrm{ECM}$ \\
\hline ERCC2 & excision repair cross-complementation group 2 & 2068 & ECM \\
\hline ERO1L & ERO1-like (S. cerevisiae) & 30001 & ECM \\
\hline ERO1LB & ERO1-like beta (S. cerevisiae) & 56605 & ECM \\
\hline ETS1 & v-ets avian erythroblastosis virus E26 oncogene homolog 1 & 2113 & $\mathrm{ECM}$ \\
\hline F11R & F11 receptor & 50848 & ECM \\
\hline FAP & fibroblast activation protein, alpha & 2191 & ECM \\
\hline FBLN1 & fibulin 1 & 2192 & ECM \\
\hline FBLN2 & fibulin 2 & 2199 & ECM \\
\hline FBLN5 & fibulin 5 & 10516 & ECM \\
\hline FBN1 & fibrillin 1 & 2200 & ECM \\
\hline FER & fer (fps/fes related) tyrosine kinase & 2241 & ECM \\
\hline FGF2 & fibroblast growth factor 2 (basic) & 2247 & $\mathrm{ECM}$ \\
\hline FKBP9 & FK506 binding protein 9, $63 \mathrm{kDa}$ & 360132 & ECM \\
\hline FMOD & Fibromodulin & 2331 & $\mathrm{ECM}$ \\
\hline FN1 & fibronectin 1 & 2335 & ECM \\
\hline FURIN & furin (paired basic amino acid cleaving enzyme) & 5045 & $\mathrm{ECM}$ \\
\hline FZD4 & frizzled class receptor 4 & 8322 & ECM \\
\hline GAS6 & growth arrest-specific 6 & 2621 & ECM \\
\hline GFAP & glial fibrillary acidic protein & 2670 & ECM \\
\hline GFOD2 & glucose-fructose oxidoreductase domain containing 2 & 81577 & $\mathrm{ECM}$ \\
\hline GPC1 & glypican 1 & 2817 & ECM \\
\hline GPM6B & glycoprotein M6B & 2824 & $\mathrm{ECM}$ \\
\hline HAS2 & hyaluronan synthase 2 & 3037 & ECM \\
\hline HAS3 & hyaluronan synthase 3 & 3038 & $\mathrm{ECM}$ \\
\hline HSD17B12 & hydroxysteroid (17-beta) dehydrogenase 12 & 51144 & ECM \\
\hline HSPG2 & heparan sulfate proteoglycan 2 & 3339 & ECM \\
\hline ICAM1 & intercellular adhesion molecule 1 & 3383 & ECM \\
\hline ICAM2 & intercellular adhesion molecule 2 & 3384 & $\mathrm{ECM}$ \\
\hline ICAM3 & intercellular adhesion molecule 3 & 3385 & ECM \\
\hline ITGA1 & integrin, alpha 1 & 3672 & $\mathrm{ECM}$ \\
\hline ITGA11 & integrin, alpha 11 & 22801 & ECM \\
\hline ITGA2 & integrin, alpha 2 (CD49B, alpha 2 subunit of VLA-2 receptor) & 3673 & ECM \\
\hline ITGA2B & integrin, alpha 2b (platelet glycoprotein IIb of IIb/IIla complex, antigen CD41) & 3674 & ECM \\
\hline ITGA3 & integrin, alpha 3 (antigen CD49C, alpha 3 subunit of VLA-3 receptor) & 3675 & ECM \\
\hline ITGA4 & integrin, alpha 4 (antigen CD49D, alpha 4 subunit of VLA-4 receptor) & 3676 & ECM \\
\hline ITGA5 & integrin, alpha 5 (fibronectin receptor, alpha polypeptide) & 3678 & ECM \\
\hline ITGA6 & integrin, alpha 6 & 3655 & ECM \\
\hline ITGA7 & integrin, alpha 7 & 3679 & ECM \\
\hline ITGA8 & integrin, alpha 8 & 8516 & ECM \\
\hline ITGA9 & integrin, alpha 9 & 3680 & ECM \\
\hline ITGAD & integrin, alpha $D$ & 3681 & ECM \\
\hline ITGAE & $\begin{array}{l}\text { integrin, alpha E (antigen CD103, human mucosal lymphocyte antigen 1; alpha } \\
\text { polypeptide) }\end{array}$ & 3682 & $\mathrm{ECM}$ \\
\hline ITGAL & $\begin{array}{l}\text { integrin, alpha L (antigen CD11A ( } \mathrm{p} 180 \text { ), lymphocyte function-associated } \\
\text { antigen 1; alpha polypeptide) }\end{array}$ & 3683 & $\mathrm{ECM}$ \\
\hline ITGAM & integrin, alpha $\mathrm{M}$ (complement component 3 receptor 3 subunit) & 3684 & ECM \\
\hline ITGAV & integrin, alpha $\mathrm{V}$ & 3685 & $\mathrm{ECM}$ \\
\hline ITGAX & integrin, alpha $\mathrm{X}$ (complement component 3 receptor 4 subunit) & 3687 & $\mathrm{ECM}$ \\
\hline ITGB2 & integrin, beta 2 (complement component 3 receptor 3 and 4 subunit) & 3689 & ECM \\
\hline ITGB3 & integrin, beta 3 (platelet glycoprotein IIla, antigen CD61) & 3690 & ECM \\
\hline ITGB4 & integrin, beta 4 & 3691 & $\mathrm{ECM}$ \\
\hline ITGB5 & integrin, beta 5 & 3693 & $\mathrm{ECM}$ \\
\hline ITGB7 & integrin, beta 7 & 3695 & $\mathrm{ECM}$ \\
\hline JAM2 & junctional adhesion molecule 2 & 58494 & ECM \\
\hline JAM3 & junctional adhesion molecule 3 & 83700 & $\mathrm{ECM}$ \\
\hline KAZALD1 & Kazal-type serine peptidase inhibitor domain 1 & 81621 & ECM \\
\hline KDR & kinase insert domain receptor & 3791 & ECM \\
\hline KIF9 & kinesin family member 9 & 64147 & ECM \\
\hline LAMA2 & laminin, alpha 2 & 3908 & $\mathrm{ECM}$ \\
\hline LAMA3 & laminin, alpha 3 & 3909 & ECM \\
\hline LAMA5 & laminin, alpha 5 & 3911 & $\mathrm{ECM}$ \\
\hline LAMB1 & laminin, beta 1 & 3912 & ECM \\
\hline LAMB2 & laminin, beta 2 (laminin S) & 3913 & $\mathrm{ECM}$ \\
\hline LAMB3 & laminin, beta 3 & 3914 & ECM \\
\hline LAMC1 & laminin, gamma 1 (formerly LAMB2) & 3915 & ECM \\
\hline LAMC3 & laminin, gamma 3 & 10319 & $\mathrm{ECM}$ \\
\hline
\end{tabular}




\begin{tabular}{|c|c|c|c|}
\hline LOX & lysyl oxidase & 4015 & $\mathrm{ECM}$ \\
\hline LOXL1 & lysyl oxidase-like 1 & 4016 & ECM \\
\hline LRP4 & low density lipoprotein receptor-related protein 4 & 4038 & ECM \\
\hline LRP5 & low density lipoprotein receptor-related protein 5 & 4041 & $\mathrm{ECM}$ \\
\hline LTBP1 & latent transforming growth factor beta binding protein 1 & 4052 & $\mathrm{ECM}$ \\
\hline LTBP3 & latent transforming growth factor beta binding protein 3 & 4054 & ECM \\
\hline LTBP4 & latent transforming growth factor beta binding protein 4 & 8425 & ECM \\
\hline LUM & Lumican & 4060 & $\mathrm{ECM}$ \\
\hline MADCAM1 & mucosal vascular addressin cell adhesion molecule 1 & 8174 & $\mathrm{ECM}$ \\
\hline MATN2 & matrilin 2 & 4147 & ECM \\
\hline MATN4 & matrilin 4 & 8785 & $\mathrm{ECM}$ \\
\hline MFAP2 & microfibrillar-associated protein 2 & 4237 & ECM \\
\hline MFAP3 & microfibrillar-associated protein 3 & 4238 & $\mathrm{ECM}$ \\
\hline MFAP4 & microfibrillar-associated protein 4 & 4239 & $\mathrm{ECM}$ \\
\hline MFAP5 & microfibrillar associated protein 5 & 8076 & ECM \\
\hline MFI2 & antigen p97 (melanoma associated) & 4241 & $\mathrm{ECM}$ \\
\hline MMP14 & matrix metallopeptidase 14 (membrane-inserted) & 4323 & $\mathrm{ECM}$ \\
\hline MMP15 & matrix metallopeptidase 15 (membrane-inserted) & 4324 & $\mathrm{ECM}$ \\
\hline MMP19 & matrix metallopeptidase 19 & 4327 & $\mathrm{ECM}$ \\
\hline MMP2 & matrix metallopeptidase 2 & 4313 & $\mathrm{ECM}$ \\
\hline MMP7 & matrix metallopeptidase 7 & 4316 & ECM \\
\hline MMP9 & matrix metallopeptidase 9 & 4318 & $\mathrm{ECM}$ \\
\hline MPZL3 & myelin protein zero-like 3 & 196264 & ECM \\
\hline NBL1 & neuroblastoma 1 , DAN family BMP antagonist & 4681 & $\mathrm{ECM}$ \\
\hline NCAM1 & neural cell adhesion molecule 1 & 4684 & ECM \\
\hline NCSTN & Nicastrin & 23385 & $\mathrm{ECM}$ \\
\hline NDNF & neuron-derived neurotrophic factor & 79625 & ECM \\
\hline NFKB2 & nuclear factor of kappa light polypeptide gene enhancer in B-cells 2 & 4791 & $\mathrm{ECM}$ \\
\hline NID1 & nidogen 1 & 4811 & ECM \\
\hline NID2 & nidogen 2 (osteonidogen) & 22795 & ECM \\
\hline NOTCH1 & notch 1 & 4851 & ECM \\
\hline NPHP3 & nephronophthisis 3 (adolescent) & 27031 & $\mathrm{ECM}$ \\
\hline NPNT & Nephronectin & 255743 & $\mathrm{ECM}$ \\
\hline NTN4 & netrin 4 & 59277 & $\mathrm{ECM}$ \\
\hline OGN & Osteoglycin & 4969 & ECM \\
\hline OLFML2A & olfactomedin-like $2 \mathrm{~A}$ & 169611 & ECM \\
\hline OLFML2B & olfactomedin-like 2B & 25903 & ECM \\
\hline $\mathrm{P} 3 \mathrm{H} 2$ & prolyl 3-hydroxylase 2 & 55214 & ECM \\
\hline P3H3 & prolyl 3-hydroxylase 3 & 10536 & ECM \\
\hline P4HA1 & prolyl 4-hydroxylase, alpha polypeptide I & 5033 & ECM \\
\hline PCOLCE & procollagen C-endopeptidase enhancer & 5118 & ECM \\
\hline PDGFA & platelet-derived growth factor alpha polypeptide & 5154 & ECM \\
\hline PDGFB & platelet-derived growth factor beta polypeptide & 5155 & ECM \\
\hline PDGFRA & platelet-derived growth factor receptor, alpha polypeptide & 5156 & ECM \\
\hline PDIA4 & protein disulfide isomerase family $\mathrm{A}$, member 4 & 9601 & ECM \\
\hline PECAM1 & platelet/endothelial cell adhesion molecule 1 & 5175 & ECM \\
\hline PLEC & Plectin & 5339 & $\mathrm{ECM}$ \\
\hline PLOD1 & procollagen-lysine, 2-oxoglutarate 5-dioxygenase 1 & 5351 & $\mathrm{ECM}$ \\
\hline PLOD2 & procollagen-lysine, 2-oxoglutarate 5-dioxygenase 2 & 5352 & ECM \\
\hline POSTN & periostin, osteoblast specific factor & 10631 & ECM \\
\hline PPIB & peptidylprolyl isomerase B (cyclophilin B) & 5479 & $\mathrm{ECM}$ \\
\hline PRDX4 & peroxiredoxin 4 & 10549 & $\mathrm{ECM}$ \\
\hline PRELP & proline/arginine-rich end leucine-rich repeat protein & 5549 & ECM \\
\hline PRG4 & proteoglycan 4 & 10216 & ECM \\
\hline PRKCA & protein kinase C, alpha & 5578 & $\mathrm{ECM}$ \\
\hline PSEN1 & presenilin 1 & 5663 & $\mathrm{ECM}$ \\
\hline PTPRS & protein tyrosine phosphatase, receptor type, $\mathrm{S}$ & 5802 & ECM \\
\hline PXDN & Peroxidasin & 7837 & ECM \\
\hline RECK & reversion-inducing-cysteine-rich protein with kazal motifs & 8434 & $\mathrm{ECM}$ \\
\hline RGCC & regulator of cell cycle & 28984 & ECM \\
\hline SDC1 & syndecan 1 & 6382 & $\mathrm{ECM}$ \\
\hline SDC3 & syndecan 3 & 9672 & ECM \\
\hline SDC4 & syndecan 4 & 6385 & $\mathrm{ECM}$ \\
\hline SERAC1 & serine active site containing 1 & 84947 & $\mathrm{ECM}$ \\
\hline SERPINE1 & $\begin{array}{l}\text { serpin peptidase inhibitor, clade } E \text { (nexin, plasminogen activator inhibitor type } \\
\text { 1), member } 1\end{array}$ & 5054 & ECM \\
\hline SERPINH1 & serpin peptidase inhibitor, clade $\mathrm{H}$ (heat shock protein 47 ), member 1 & 871 & $\mathrm{ECM}$ \\
\hline SH3PXD2B & SH3 and $\mathrm{PX}$ domains $2 \mathrm{~B}$ & 285590 & ECM \\
\hline SMOC1 & SPARC related modular calcium binding 1 & 64093 & $\mathrm{ECM}$ \\
\hline SMOC2 & SPARC related modular calcium binding 2 & 64094 & $\mathrm{ECM}$ \\
\hline SOX9 & SRY (sex determining region Y)-box 9 & 6662 & $\mathrm{ECM}$ \\
\hline
\end{tabular}




\begin{tabular}{|c|c|c|c|}
\hline SPARCL1 & SPARC-like 1 (hevin) & 8404 & ECM \\
\hline SPINT1 & serine peptidase inhibitor, Kunitz type 1 & 6692 & $\mathrm{ECM}$ \\
\hline SPOCK2 & sparc/osteonectin, cwcv and kazal-like domains proteoglycan (testican) 2 & 9806 & $\mathrm{ECM}$ \\
\hline SPON1 & spondin 1 , extracellular matrix protein & 10418 & $\mathrm{ECM}$ \\
\hline SPON2 & spondin 2, extracellular matrix protein & 10417 & $\mathrm{ECM}$ \\
\hline SPP1 & secreted phosphoprotein 1 & 6696 & $\mathrm{ECM}$ \\
\hline TCF15 & transcription factor 15 (basic helix-loop-helix) & 6939 & ECM \\
\hline TGFB1 & transforming growth factor, beta 1 & 7040 & $\mathrm{ECM}$ \\
\hline TGFB2 & transforming growth factor, beta 2 & 7042 & $\mathrm{ECM}$ \\
\hline TGFB3 & transforming growth factor, beta 3 & 7043 & $\mathrm{ECM}$ \\
\hline TGFBI & transforming growth factor, beta-induced, $68 \mathrm{kDa}$ & 7045 & $\mathrm{ECM}$ \\
\hline TGFBR3 & transforming growth factor, beta receptor III & 7049 & $\mathrm{ECM}$ \\
\hline THBS1 & thrombospondin 1 & 7057 & $\mathrm{ECM}$ \\
\hline THBS2 & thrombospondin 2 & 7058 & $\mathrm{ECM}$ \\
\hline THBS3 & thrombospondin 3 & 7059 & $\mathrm{ECM}$ \\
\hline TIMP1 & TIMP metallopeptidase inhibitor 1 & 7076 & $\mathrm{ECM}$ \\
\hline TIMP2 & TIMP metallopeptidase inhibitor 2 & 7077 & $\mathrm{ECM}$ \\
\hline TIMP4 & TIMP metallopeptidase inhibitor 4 & 7079 & $\mathrm{ECM}$ \\
\hline TLL1 & tolloid-like 1 & 7092 & $\mathrm{ECM}$ \\
\hline TNC & tenascin C & 3371 & $\mathrm{ECM}$ \\
\hline TNF & tumor necrosis factor & 7124 & ECM \\
\hline TNFRSF11B & tumor necrosis factor receptor superfamily, member $11 b$ & 4982 & $\mathrm{ECM}$ \\
\hline TNN & tenascin $\mathrm{N}$ & 63923 & $\mathrm{ECM}$ \\
\hline TNXB & tenascin XB & 7148 & $\mathrm{ECM}$ \\
\hline TRAPPC4 & trafficking protein particle complex 4 & 51399 & $\mathrm{ECM}$ \\
\hline VCAM1 & vascular cell adhesion molecule 1 & 7412 & $\mathrm{ECM}$ \\
\hline VCAN & Versican & 1462 & $\mathrm{ECM}$ \\
\hline VIT & Vitrin & 5212 & $\mathrm{ECM}$ \\
\hline VWA1 & von Willebrand factor A domain containing 1 & 64856 & $\mathrm{ECM}$ \\
\hline VWF & von Willebrand factor & 7450 & ECM \\
\hline
\end{tabular}


Supplement Table 2: Correlations between stress- and ECM-related genes, which correlated with the weight regain percentage, during the WL, WS and DI period.

\begin{tabular}{|c|c|c|c|c|}
\hline Stress genes & ECM genes & $R$ & P-value & Period \\
\hline ALDH2 & APBB2 & 0.738 & $<0.001$ & WL \\
\hline ALDH2 & DMD & 0.686 & $<0.001$ & WL \\
\hline ALDH2 & DDR2 & 0.789 & $<0.001$ & WL \\
\hline ANXA5 & CNTNAP1 & -0.682 & $<0.001$ & WL \\
\hline CRYAB & DMD & 0.728 & $<0.001$ & WL \\
\hline CRYAB & FBLN2 & 0.601 & 0.003 & WL \\
\hline CRYAB & EFEMP1 & 0.616 & 0.002 & WL \\
\hline CRYAB & DDR2 & 0.728 & $<0.001$ & WL \\
\hline CRYAB & CCDC80 & 0.601 & 0.002 & WL \\
\hline PGK1 & BMP2 & -0.641 & 0.001 & WL \\
\hline PRDX6 & DMD & 0.643 & 0.001 & WL \\
\hline PRDX6 & EFEMP1 & 0.623 & 0.001 & WL \\
\hline PRDX6 & CNTNAP1 & -0.624 & 0.001 & WL \\
\hline PTK2B & ITGB7 & 0.616 & 0.002 & WL \\
\hline PTK2B & ADAM19 & 0.657 & 0.001 & WL \\
\hline RGS14 & ITGB7 & 0.617 & 0.002 & WL \\
\hline RGS14 & ADAM19 & 0.763 & $<0.001$ & WL \\
\hline SQSTM1 & EFEMP1 & 0.603 & 0.002 & WL \\
\hline ACTB & ACTN1 & 0.729 & $<0.001$ & WS \\
\hline ACTB & CAPN1 & 0.67 & $<0.001$ & WS \\
\hline АCTB & DMD & -0.617 & 0.001 & WS \\
\hline ACTB & ITGA3 & 0.768 & $<0.001$ & WS \\
\hline ACTB & ITGA5 & 0.726 & $<0.001$ & WS \\
\hline АСТВ & PDIA4 & 0.664 & $<0.001$ & WS \\
\hline ACTB & COLGALT1 & 0.64 & 0.001 & WS \\
\hline AKR1B1 & CAPN1 & 0.767 & $<0.001$ & WS \\
\hline AKR1B1 & CTSB & 0.708 & $<0.001$ & WS \\
\hline AKR1B1 & ITGA3 & 0.753 & $<0.001$ & WS \\
\hline AKR1B1 & ITGAX & 0.698 & $<0.001$ & WS \\
\hline AKR1B1 & PLOD1 & 0.715 & $<0.001$ & WS \\
\hline AKR1B1 & PPIB & 0.694 & $<0.001$ & WS \\
\hline AKR1B1 & SPINT1 & 0.741 & $<0.001$ & WS \\
\hline AKR1B1 & PDIA4 & 0.731 & $<0.001$ & WS \\
\hline AKR1B1 & NCSTN & 0.611 & 0.002 & WS \\
\hline AKR1B1 & COLGALT1 & 0.635 & 0.001 & WS \\
\hline AKR1B1 & EMILIN2 & 0.714 & $<0.001$ & WS \\
\hline ALDOA & ACTN1 & 0.602 & 0.002 & WS \\
\hline ALDOA & CTSB & 0.728 & $<0.001$ & WS \\
\hline ALDOA & ITGB5 & 0.692 & $<0.001$ & WS \\
\hline ALDOA & PLOD1 & 0.732 & $<0.001$ & WS \\
\hline ALDOA & PPIB & 0.67 & $<0.001$ & WS \\
\hline ALDOA & SPARCL1 & -0.779 & $<0.001$ & WS \\
\hline ALDOA & NCSTN & 0.702 & $<0.001$ & WS \\
\hline ALDOA & COLGALT1 & 0.602 & 0.002 & WS \\
\hline ALDOA & EMILIN2 & 0.628 & 0.001 & WS \\
\hline ARHGDIA & ACTN1 & 0.634 & 0.001 & WS \\
\hline ARHGDIA & CAPN1 & 0.616 & 0.001 & WS \\
\hline ARHGDIA & CTSB & 0.707 & $<0.001$ & WS \\
\hline ARHGDIA & ITGA3 & 0.625 & 0.001 & WS \\
\hline ARHGDIA & ITGB5 & 0.681 & $<0.001$ & WS \\
\hline ARHGDIA & PLOD1 & 0.668 & $<0.001$ & WS \\
\hline ARHGDIA & PPIB & 0.606 & 0.002 & WS \\
\hline ARHGDIA & SPINT1 & 0.622 & 0.001 & WS \\
\hline ARHGDIA & SPARCL1 & -0.797 & $<0.001$ & WS \\
\hline ARHGDIA & NCSTN & 0.601 & 0.002 & WS \\
\hline ARHGDIA & EMILIN2 & 0.64 & 0.001 & WS \\
\hline BCLAF1 & VIT & 0.687 & $<0.001$ & WS \\
\hline C5 & VIT & 0.722 & $<0.001$ & WS \\
\hline CALR & ACTN1 & 0.646 & 0.001 & WS \\
\hline CALR & CAPN1 & 0.808 & $<0.001$ & WS \\
\hline CALR & CTSB & 0.814 & $<0.001$ & WS \\
\hline CALR & DMD & -0.674 & $<0.001$ & WS \\
\hline CALR & $\mathrm{ECM} 2$ & -0.625 & 0.001 & WS \\
\hline CALR & ITGA3 & 0.783 & $<0.001$ & WS \\
\hline CALR & ITGAM & 0.641 & 0.001 & WS \\
\hline CALR & ITGAX & 0.601 & 0.002 & WS \\
\hline CALR & ITGB5 & 0.714 & $<0.001$ & WS \\
\hline
\end{tabular}




\begin{tabular}{|c|c|c|c|c|}
\hline CALR & PLOD1 & 0.856 & $<0.001$ & WS \\
\hline CALR & PPIB & 0.832 & $<0.001$ & WS \\
\hline CALR & SPINT1 & 0.71 & $<0.001$ & WS \\
\hline CALR & SPARCL1 & -0.611 & 0.002 & WS \\
\hline CALR & PDIA4 & 0.872 & $<0.001$ & WS \\
\hline CALR & NCSTN & 0.706 & $<0.001$ & WS \\
\hline CALR & COLGALT1 & 0.745 & $<0.001$ & WS \\
\hline CALR & EMILIN2 & 0.82 & $<0.001$ & WS \\
\hline CFL1 & ACTN1 & 0.781 & $<0.001$ & WS \\
\hline CFL1 & CAPN1 & 0.808 & $<0.001$ & WS \\
\hline CFL1 & CTSB & 0.861 & $<0.001$ & WS \\
\hline CFL1 & CTSS & 0.728 & $<0.001$ & WS \\
\hline CFL1 & DMD & -0.679 & $<0.001$ & WS \\
\hline CFL1 & ECM2 & -0.726 & $<0.001$ & WS \\
\hline CFL1 & ITGA3 & 0.859 & $<0.001$ & WS \\
\hline CFL1 & ITGA5 & 0.657 & $<0.001$ & WS \\
\hline CFL1 & ITGAM & 0.77 & $<0.001$ & WS \\
\hline CFL1 & ITGAX & 0.679 & $<0.001$ & WS \\
\hline CFL1 & ITGB2 & 0.653 & 0.001 & WS \\
\hline CFL1 & ITGB5 & 0.703 & $<0.001$ & WS \\
\hline CFL1 & PLOD1 & 0.863 & $<0.001$ & WS \\
\hline CFL1 & PLOD2 & -0.622 & 0.001 & WS \\
\hline CFL1 & PPIB & 0.842 & $<0.001$ & WS \\
\hline CFL1 & SPINT1 & 0.633 & 0.001 & WS \\
\hline CFL1 & SPARCL1 & -0.738 & $<0.001$ & WS \\
\hline CFL1 & PDIA4 & 0.746 & $<0.001$ & WS \\
\hline CFL1 & NCSTN & 0.818 & $<0.001$ & WS \\
\hline CFL1 & COLGALT1 & 0.807 & $<0.001$ & WS \\
\hline CFL1 & EMILIN2 & 0.778 & $<0.001$ & WS \\
\hline CLIC1 & CTSB & 0.703 & $<0.001$ & WS \\
\hline CLIC1 & DMD & -0.621 & 0.001 & WS \\
\hline CLIC1 & ITGA3 & 0.608 & 0.002 & WS \\
\hline CLIC1 & ITGAM & 0.605 & 0.002 & WS \\
\hline CLIC1 & ITGAX & 0.622 & 0.001 & WS \\
\hline CLIC1 & ITGB5 & 0.71 & $<0.001$ & WS \\
\hline CLIC1 & PPIB & 0.648 & 0.001 & WS \\
\hline CLIC1 & PDIA4 & 0.679 & $<0.001$ & WS \\
\hline CLIC1 & EMILIN2 & 0.766 & $<0.001$ & WS \\
\hline ENO1 & ACTN1 & 0.709 & $<0.001$ & WS \\
\hline ENO1 & CAPN1 & 0.635 & 0.001 & WS \\
\hline ENO1 & CTSB & 0.73 & $<0.001$ & WS \\
\hline ENO1 & ITGA3 & 0.663 & $<0.001$ & WS \\
\hline ENO1 & ITGA5 & 0.69 & $<0.001$ & WS \\
\hline ENO1 & ITGB5 & 0.713 & $<0.001$ & WS \\
\hline ENO1 & PLOD1 & 0.728 & $<0.001$ & WS \\
\hline ENO1 & PPIB & 0.728 & $<0.001$ & WS \\
\hline ENO1 & SPARCL1 & -0.713 & $<0.001$ & WS \\
\hline ENO1 & PDIA4 & 0.673 & $<0.001$ & WS \\
\hline ENO1 & NCSTN & 0.772 & $<0.001$ & WS \\
\hline ENO1 & COLGALT1 & 0.718 & $<0.001$ & WS \\
\hline ENO1 & EMILIN2 & 0.67 & $<0.001$ & WS \\
\hline HSPA5 & ACTN1 & 0.671 & $<0.001$ & WS \\
\hline HSPA5 & CTSB & 0.609 & 0.002 & WS \\
\hline HSPA5 & DMD & -0.632 & 0.001 & WS \\
\hline HSPA5 & ITGA3 & 0.726 & $<0.001$ & WS \\
\hline HSPA5 & PDIA4 & 0.632 & 0.001 & WS \\
\hline HSPA5 & COLGALT1 & 0.609 & 0.002 & WS \\
\hline HSPA8 & ACTN1 & 0.723 & $<0.001$ & WS \\
\hline HSPA8 & PPIB & 0.673 & $<0.001$ & WS \\
\hline HSPB1 & ACTN1 & 0.744 & $<0.001$ & WS \\
\hline HSPB1 & ITGA5 & 0.618 & 0.001 & WS \\
\hline HYOU1 & ACTN1 & 0.769 & $<0.001$ & WS \\
\hline HYOU1 & CAPN1 & 0.601 & 0.002 & WS \\
\hline HYOU1 & CTSB & 0.629 & 0.001 & WS \\
\hline HYOU1 & GPC1 & 0.62 & 0.001 & WS \\
\hline HYOU1 & ITGA3 & 0.777 & $<0.001$ & WS \\
\hline HYOU1 & ITGA5 & 0.747 & $<0.001$ & WS \\
\hline HYOU1 & PLOD1 & 0.756 & $<0.001$ & WS \\
\hline HYOU1 & PPIB & 0.657 & $<0.001$ & WS \\
\hline HYOU1 & PDIA4 & 0.7 & $<0.001$ & WS \\
\hline HYOU1 & NCSTN & 0.713 & $<0.001$ & WS \\
\hline
\end{tabular}




\begin{tabular}{|c|c|c|c|c|}
\hline HYOU1 & COLGALT1 & 0.742 & $<0.001$ & WS \\
\hline MAPK13 & ACTN1 & 0.72 & $<0.001$ & WS \\
\hline MAPK13 & CAPN1 & 0.766 & $<0.001$ & WS \\
\hline MAPK13 & CTSB & 0.854 & $<0.001$ & WS \\
\hline MAPK13 & CTSS & 0.721 & $<0.001$ & WS \\
\hline MAPK13 & DMD & -0.682 & $<0.001$ & WS \\
\hline MAPK13 & ECM2 & -0.658 & $<0.001$ & WS \\
\hline MAPK13 & ITGA3 & 0.781 & $<0.001$ & WS \\
\hline MAPK13 & ITGAL & 0.602 & 0.002 & WS \\
\hline MAPK13 & ITGAM & 0.766 & $<0.001$ & WS \\
\hline MAPK13 & ITGAX & 0.676 & $<0.001$ & WS \\
\hline MAPK13 & PLOD1 & 0.73 & $<0.001$ & WS \\
\hline MAPK13 & PPIB & 0.79 & $<0.001$ & WS \\
\hline MAPK13 & PDIA4 & 0.702 & $<0.001$ & WS \\
\hline MAPK13 & NCSTN & 0.752 & $<0.001$ & WS \\
\hline MAPK13 & COLGALT1 & 0.653 & 0.001 & WS \\
\hline MAPK13 & EMILIN2 & 0.779 & $<0.001$ & WS \\
\hline MAPK8 & CAPN1 & -0.602 & 0.002 & WS \\
\hline MAPK8 & ECM2 & 0.772 & $<0.001$ & WS \\
\hline MAPK8 & GPC1 & -0.631 & 0.001 & WS \\
\hline MAPK8 & ITGA5 & -0.687 & $<0.001$ & WS \\
\hline MAPK8 & KDR & 0.652 & 0.001 & WS \\
\hline MAPK8 & SPARCL1 & 0.624 & 0.001 & WS \\
\hline MAPKAPK3 & CAPN1 & 0.736 & $<0.001$ & WS \\
\hline MAPKAPK3 & CTSB & 0.617 & 0.001 & WS \\
\hline MAPKAPK3 & CTSS & 0.698 & $<0.001$ & WS \\
\hline MAPKAPK3 & ECM2 & -0.711 & $<0.001$ & WS \\
\hline MAPKAPK3 & ITGA3 & 0.635 & 0.001 & WS \\
\hline MAPKAPK3 & ITGAL & 0.614 & 0.001 & WS \\
\hline MAPKAPK3 & ITGAM & 0.738 & $<0.001$ & WS \\
\hline MAPKAPK3 & ITGAX & 0.753 & $<0.001$ & WS \\
\hline MAPKAPK3 & ITGB2 & 0.683 & $<0.001$ & WS \\
\hline MAPKAPK3 & KDR & -0.746 & $<0.001$ & WS \\
\hline MAPKAPK3 & PPIB & 0.662 & $<0.001$ & WS \\
\hline MAPKAPK3 & SPINT1 & 0.678 & $<0.001$ & WS \\
\hline MAPKAPK3 & SPARCL1 & -0.766 & $<0.001$ & WS \\
\hline MAPKAPK3 & NCSTN & 0.609 & 0.002 & WS \\
\hline MAPKAPK3 & COLGALT1 & 0.607 & 0.002 & WS \\
\hline MAPKAPK3 & EMILIN2 & 0.677 & $<0.001$ & WS \\
\hline MEF2C & ITGB5 & -0.65 & 0.001 & WS \\
\hline MEF2C & KDR & 0.687 & $<0.001$ & WS \\
\hline MEF2C & SPARCL1 & 0.69 & $<0.001$ & WS \\
\hline OMA1 & CAPN1 & -0.617 & 0.001 & WS \\
\hline OMA1 & ECM2 & 0.683 & $<0.001$ & WS \\
\hline OMA1 & ITGA5 & -0.671 & $<0.001$ & WS \\
\hline OMA1 & SPARCL1 & 0.613 & 0.002 & WS \\
\hline PGK1 & ACTN1 & 0.628 & 0.001 & WS \\
\hline PGK1 & CTSS & 0.711 & $<0.001$ & WS \\
\hline PGK1 & DMD & -0.627 & 0.001 & WS \\
\hline PGK1 & ITGAL & 0.612 & 0.001 & WS \\
\hline PGK1 & ITGAM & 0.728 & $<0.001$ & WS \\
\hline PGK1 & ITGAX & 0.605 & 0.002 & WS \\
\hline PGK1 & ITGB2 & 0.692 & $<0.001$ & WS \\
\hline PGK1 & PLOD1 & 0.686 & $<0.001$ & WS \\
\hline PGK1 & PPIB & 0.611 & 0.002 & WS \\
\hline PGK1 & SPARCL1 & -0.656 & 0.001 & WS \\
\hline PKM & CAPN1 & 0.909 & $<0.001$ & WS \\
\hline PKM & CTSB & 0.829 & $<0.001$ & WS \\
\hline PKM & CTSS & 0.693 & $<0.001$ & WS \\
\hline PKM & DMD & -0.685 & $<0.001$ & WS \\
\hline PKM & ECM2 & -0.692 & $<0.001$ & WS \\
\hline PKM & ITGA3 & 0.913 & $<0.001$ & WS \\
\hline PKM & ITGA5 & 0.748 & $<0.001$ & WS \\
\hline PKM & ITGAL & 0.66 & $<0.001$ & WS \\
\hline PKM & ITGAM & 0.796 & $<0.001$ & WS \\
\hline PKM & ITGAX & 0.745 & $<0.001$ & WS \\
\hline PKM & ITGB2 & 0.678 & $<0.001$ & WS \\
\hline PKM & ITGB5 & 0.714 & $<0.001$ & WS \\
\hline PKM & PLOD1 & 0.752 & $<0.001$ & WS \\
\hline PKM & PLOD2 & -0.691 & $<0.001$ & WS \\
\hline PKM & PPIB & 0.805 & $<0.001$ & WS \\
\hline
\end{tabular}




\begin{tabular}{|c|c|c|c|c|}
\hline PKM & SPINT1 & 0.673 & $<0.001$ & WS \\
\hline PKM & SPARCL1 & -0.653 & 0.001 & WS \\
\hline PKM & PDIA4 & 0.852 & $<0.001$ & WS \\
\hline PKM & NCSTN & 0.718 & $<0.001$ & WS \\
\hline PKM & COLGALT1 & 0.836 & $<0.001$ & WS \\
\hline PKM & EMILIN2 & 0.868 & $<0.001$ & WS \\
\hline PTEN & $\mathrm{ECM} 2$ & 0.625 & 0.001 & WS \\
\hline PTEN & NCAM1 & 0.606 & 0.002 & WS \\
\hline PTK2B & CAPN1 & 0.63 & 0.001 & WS \\
\hline PTK2B & $\mathrm{ECM} 2$ & -0.656 & $<0.001$ & WS \\
\hline PTK2B & ITGAL & 0.726 & $<0.001$ & WS \\
\hline PTK2B & ITGAM & 0.67 & $<0.001$ & WS \\
\hline PTK2B & ITGAX & 0.692 & $<0.001$ & WS \\
\hline PTK2B & ITGB2 & 0.651 & 0.001 & WS \\
\hline PTK2B & PLOD2 & -0.606 & 0.002 & WS \\
\hline RGS14 & ICAM3 & 0.834 & $<0.001$ & WS \\
\hline RGS14 & ITGAL & 0.635 & 0.001 & WS \\
\hline RPS6KA1 & CAPN1 & 0.808 & $<0.001$ & WS \\
\hline RPS6KA1 & CTSB & 0.685 & $<0.001$ & WS \\
\hline RPS6KA1 & CTSS & 0.864 & $<0.001$ & WS \\
\hline RPS6KA1 & DMD & -0.735 & $<0.001$ & WS \\
\hline RPS6KA1 & ECM2 & -0.772 & $<0.001$ & WS \\
\hline RPS6KA1 & ITGA3 & 0.736 & $<0.001$ & WS \\
\hline RPS6KA1 & ITGA5 & 0.605 & 0.002 & WS \\
\hline RPS6KA1 & ITGAL & 0.823 & $<0.001$ & WS \\
\hline RPS6KA1 & ITGAM & 0.913 & $<0.001$ & WS \\
\hline RPS6KA1 & ITGAX & 0.918 & $<0.001$ & WS \\
\hline RPS6KA1 & ITGB2 & 0.892 & $<0.001$ & WS \\
\hline RPS6KA1 & PLOD2 & -0.685 & $<0.001$ & WS \\
\hline RPS6KA1 & PPIB & 0.634 & 0.001 & WS \\
\hline RPS6KA1 & SPINT1 & 0.683 & $<0.001$ & WS \\
\hline RPS6KA1 & SPARCL1 & -0.662 & 0.001 & WS \\
\hline RPS6KA1 & PDIA4 & 0.638 & 0.001 & WS \\
\hline RPS6KA1 & COLGALT1 & 0.607 & 0.002 & WS \\
\hline RPS6KA1 & EMILIN2 & 0.798 & $<0.001$ & WS \\
\hline ZAK & ACTN1 & -0.634 & 0.001 & WS \\
\hline ZAK & CAPN1 & -0.783 & $<0.001$ & WS \\
\hline ZAK & CTSB & -0.621 & 0.001 & WS \\
\hline ZAK & DMD & 0.757 & $<0.001$ & WS \\
\hline ZAK & ECM2 & 0.72 & $<0.001$ & WS \\
\hline ZAK & ITGA3 & -0.765 & $<0.001$ & WS \\
\hline ZAK & ITGA5 & -0.667 & $<0.001$ & WS \\
\hline ZAK & ITGAL & -0.643 & 0.001 & WS \\
\hline ZAK & ITGAM & -0.632 & 0.001 & WS \\
\hline ZAK & ITGAX & -0.616 & 0.001 & WS \\
\hline ZAK & ITGB3 & -0.626 & 0.001 & WS \\
\hline ZAK & PLOD2 & 0.689 & $<0.001$ & WS \\
\hline ZAK & PPIB & -0.641 & 0.001 & WS \\
\hline ZAK & SPINT1 & -0.621 & 0.001 & WS \\
\hline ZAK & PDIA4 & -0.723 & $<0.001$ & WS \\
\hline ZAK & COLGALT1 & -0.636 & 0.001 & WS \\
\hline ZAK & EMILIN2 & -0.656 & 0.001 & WS \\
\hline ALDOA & BMP2 & 0.641 & 0.001 & DI \\
\hline ALDOA & BMP2 & 0.617 & 0.001 & DI \\
\hline ANXA5 & TRAPPC4 & 0.748 & $<0.001$ & DI \\
\hline ANXA5 & COL23A1 & -0.617 & 0.001 & DI \\
\hline ARHGDIA & ITGA5 & 0.615 & 0.001 & DI \\
\hline ARHGDIA & MMP15 & 0.651 & $<0.001$ & DI \\
\hline ATP5B & MMP15 & 0.631 & 0.001 & DI \\
\hline CFL1 & BMP2 & 0.663 & $<0.001$ & DI \\
\hline CFL1 & ITGA5 & 0.664 & $<0.001$ & DI \\
\hline CRHBP & ETS1 & 0.6 & 0.002 & DI \\
\hline CRHBP & FBLN2 & -0.671 & $<0.001$ & DI \\
\hline CRYAB & CD151 & 0.652 & 0.001 & DI \\
\hline CRYAB & TRAPPC4 & 0.641 & 0.001 & DI \\
\hline ENO1 & BMP2 & 0.624 & 0.001 & DI \\
\hline ENO1 & CD151 & 0.773 & $<0.001$ & DI \\
\hline ENO1 & VCAM1 & -0.626 & 0.001 & DI \\
\hline GSTP1 & BMP2 & 0.613 & 0.001 & DI \\
\hline GSTP1 & CD151 & 0.705 & $<0.001$ & DI \\
\hline LGALS1 & CD151 & 0.789 & $<0.001$ & DI \\
\hline
\end{tabular}




\begin{tabular}{llccc} 
LGALS1 & FBLN2 & 0.605 & 0.002 & DI \\
LGALS1 & ITGA7 & 0.635 & 0.001 & DI \\
LGALS1 & VCAM1 & -0.666 & $<0.001$ & DI \\
MEF2C & VCAM1 & 0.609 & 0.001 & DI \\
PHB & VCAM1 & -0.611 & 0.001 & DI \\
SOSTM1 & ITGA5 & 0.667 & $<0.001$ & DI \\
\hline
\end{tabular}

Pearson correlations $(|r| \geq 0.600 ; \mathrm{P} \leq 0.001)$ between stress- and ECM-related genes, which correlated with the weight regain percentage $(P \leq 0.05)$, during 3 periods. $W L$, weight loss; WS, weight stable; DI, dietary intervention. 
Supplement Table 3: Interactions between stress- and ECM-related genes and the effect on the weight regain percentage.

\begin{tabular}{|c|c|c|c|c|c|}
\hline Stress genes & ECM genes & $\begin{array}{c}\text { Interaction } \\
\text { P-value }\end{array}$ & Model $r^{2}$ & Model P-value & Period \\
\hline ANXA5 & ITGA4 & $3.23 \mathrm{E}-04$ & 0.638 & $3.08 \mathrm{E}-04$ & $\overline{W L}$ \\
\hline ARHGDIA & ADAM11 & $7.88 \mathrm{E}-05$ & 0.593 & 8.66E-04 & WL \\
\hline ARHGDIA & EGFLAM & $4.12 \mathrm{E}-04$ & 0.530 & $3.02 \mathrm{E}-03$ & WL \\
\hline BCLAF1 & ADAM11 & $7.68 \mathrm{E}-05$ & 0.595 & $8.26 \mathrm{E}-04$ & WL \\
\hline BCLAF1 & EGFLAM & $6.94 \mathrm{E}-04$ & 0.509 & 4.34E-03 & WL \\
\hline DDIT3 & COL6A1 & $3.38 \mathrm{E}-04$ & 0.523 & $3.37 E-03$ & WL \\
\hline DDIT3 & ECM1 & $3.91 \mathrm{E}-04$ & 0.522 & $3.44 \mathrm{E}-03$ & WL \\
\hline DDIT3 & TIMP2 & $7.44 \mathrm{E}-04$ & 0.503 & $4.81 \mathrm{E}-03$ & WL \\
\hline ENO1 & ADAM15 & $2.90 \mathrm{E}-04$ & 0.545 & $2.26 \mathrm{E}-03$ & WL \\
\hline ENO1 & EGFLAM & $9.68 \mathrm{E}-04$ & 0.486 & $6.46 \mathrm{E}-03$ & WL \\
\hline GATA6 & COL1A1 & $5.18 \mathrm{E}-04$ & 0.517 & $3.81 \mathrm{E}-03$ & WL \\
\hline GATA6 & COL6A3 & $1.16 \mathrm{E}-04$ & 0.581 & $1.12 \mathrm{E}-03$ & WL \\
\hline GATA6 & $\mathrm{MFI} 2$ & $3.95 \mathrm{E}-04$ & 0.545 & $2.25 \mathrm{E}-03$ & WL \\
\hline GATA6 & THBS3 & $9.58 \mathrm{E}-04$ & 0.477 & $7.46 \mathrm{E}-03$ & WL \\
\hline GATA6 & MADCAM1 & $2.91 \mathrm{E}-04$ & 0.608 & $6.21 \mathrm{E}-04$ & WL \\
\hline GATA6 & CSGALNACT1 & $5.00 \mathrm{E}-04$ & 0.510 & 4.32E-03 & WL \\
\hline GATA6 & KAZALD1 & $3.78 \mathrm{E}-04$ & 0.519 & $3.66 \mathrm{E}-03$ & WL \\
\hline GSTP1 & ADAMTS4 & $6.44 \mathrm{E}-04$ & 0.605 & $6.59 \mathrm{E}-04$ & WL \\
\hline GSTP1 & NPNT & $9.80 \mathrm{E}-05$ & 0.665 & $1.55 \mathrm{E}-04$ & WL \\
\hline HILPDA & NPNT & $2.05 \mathrm{E}-04$ & 0.551 & $2.01 \mathrm{E}-03$ & WL \\
\hline HSF2 & COL1A1 & $6.20 \mathrm{E}-04$ & 0.507 & 4.49E-03 & WL \\
\hline HSF2 & COL6A3 & $1.77 \mathrm{E}-04$ & 0.560 & $1.71 \mathrm{E}-03$ & WL \\
\hline HSF2 & TNC & $9.11 \mathrm{E}-04$ & 0.468 & 8.66E-03 & WL \\
\hline HSF2 & ADAM11 & $5.96 \mathrm{E}-04$ & 0.499 & $5.18 \mathrm{E}-03$ & WL \\
\hline HSF2 & PDGFA & 5.99E-04 & 0.511 & 4.19E-03 & WL \\
\hline HSF2 & MADCAM1 & $9.75 \mathrm{E}-04$ & 0.589 & $9.42 \mathrm{E}-04$ & WL \\
\hline HSF2 & EGFLAM & $2.88 \mathrm{E}-04$ & 0.547 & $2.18 \mathrm{E}-03$ & WL \\
\hline HSP90AB4P & ADAM11 & $1.16 \mathrm{E}-04$ & 0.619 & $4.82 \mathrm{E}-04$ & WL \\
\hline HSP90AB4P & $\mathrm{F} 11 \mathrm{R}$ & 4.71E-04 & 0.552 & $1.99 \mathrm{E}-03$ & WL \\
\hline HSP90AB4P & COL21A1 & 4.04E-04 & 0.567 & $1.48 \mathrm{E}-03$ & WL \\
\hline HSP90AB4P & ADAMTS14 & $2.78 \mathrm{E}-04$ & 0.577 & $1.20 \mathrm{E}-03$ & WL \\
\hline HSPD1 & CTGF & $2.58 \mathrm{E}-04$ & 0.572 & $1.33 \mathrm{E}-03$ & WL \\
\hline HSPD1 & SMOC1 & $3.09 E-04$ & 0.560 & $1.70 \mathrm{E}-03$ & WL \\
\hline HYOU1 & $\mathrm{ABI} 3 \mathrm{BP}$ & $5.63 \mathrm{E}-04$ & 0.688 & $8.34 \mathrm{E}-05$ & WL \\
\hline KLF15 & FBLN2 & $5.41 \mathrm{E}-04$ & 0.625 & $6.64 \mathrm{E}-04$ & WL \\
\hline KLF2 & ECM1 & $6.56 \mathrm{E}-04$ & 0.543 & $2.33 \mathrm{E}-03$ & WL \\
\hline KLF2 & TNXB & $1.93 \mathrm{E}-04$ & 0.621 & $4.61 \mathrm{E}-04$ & WL \\
\hline LGALS1 & ADAMTS4 & $4.66 \mathrm{E}-04$ & 0.588 & $9.61 \mathrm{E}-04$ & WL \\
\hline LGALS1 & ADAMTS1 & $7.29 \mathrm{E}-04$ & 0.581 & $1.11 \mathrm{E}-03$ & WL \\
\hline LMNA & ADAM8 & $2.31 \mathrm{E}-04$ & 0.597 & $7.82 \mathrm{E}-04$ & WL \\
\hline LMNA & EGFLAM & $8.84 \mathrm{E}-04$ & 0.502 & 4.89E-03 & WL \\
\hline MAPK11 & FN1 & $5.75 \mathrm{E}-04$ & 0.510 & $4.32 \mathrm{E}-03$ & WL \\
\hline MAPK11 & $\mathrm{MFI} 2$ & 3.59E-04 & 0.544 & $2.30 \mathrm{E}-03$ & WL \\
\hline MAPK11 & SERPINE1 & 7.19E-04 & 0.485 & $6.53 \mathrm{E}-03$ & WL \\
\hline MAPK11 & CSGALNACT1 & $1.14 \mathrm{E}-04$ & 0.582 & $1.08 \mathrm{E}-03$ & WL \\
\hline MUC1 & ITGA11 & $5.30 \mathrm{E}-04$ & 0.532 & $2.89 \mathrm{E}-03$ & WL \\
\hline PGK1 & CTGF & 8.01E-04 & 0.578 & $1.18 \mathrm{E}-03$ & WL \\
\hline PGK1 & TGFBR3 & $7.44 \mathrm{E}-04$ & 0.631 & $3.67 E-04$ & WL \\
\hline PHB & COL1A1 & $4.02 \mathrm{E}-04$ & 0.582 & $1.09 \mathrm{E}-03$ & WL \\
\hline PHB & ENG & $8.18 \mathrm{E}-04$ & 0.551 & $2.03 \mathrm{E}-03$ & WL \\
\hline PHB & ADAMTS4 & $2.74 \mathrm{E}-05$ & 0.688 & $8.42 \mathrm{E}-05$ & WL \\
\hline PHB & KAZALD1 & $9.89 \mathrm{E}-04$ & 0.549 & $2.08 \mathrm{E}-03$ & WL \\
\hline PPIA & NPNT & 7.67E-04 & 0.515 & $3.90 \mathrm{E}-03$ & WL \\
\hline PTEN & ACTN1 & $9.97 E-04$ & 0.497 & $5.38 \mathrm{E}-03$ & WL \\
\hline PTK2B & ADAMTS1 & $6.46 \mathrm{E}-04$ & 0.642 & $2.82 \mathrm{E}-04$ & WL \\
\hline RGS14 & PECAM1 & $5.03 E-04$ & 0.641 & $2.88 \mathrm{E}-04$ & WL \\
\hline TAOK2 & ITGA11 & $9.53 \mathrm{E}-04$ & 0.478 & 7.37E-03 & $W L$ \\
\hline TAOK2 & NCSTN & $8.86 \mathrm{E}-04$ & 0.497 & $5.40 \mathrm{E}-03$ & WL \\
\hline TRAF2 & ITGA11 & 7.07E-04 & 0.497 & $5.41 E-03$ & WL \\
\hline UCN2 & CSGALNACT1 & $9.13 \mathrm{E}-04$ & 0.481 & $7.06 \mathrm{E}-03$ & WL \\
\hline $\mathrm{ALDH} 2$ & MPZL3 & $6.80 \mathrm{E}-04$ & 0.510 & $3.06 \mathrm{E}-03$ & WS \\
\hline ANXA2 & VWF & $9.81 \mathrm{E}-04$ & 0.471 & $6.11 \mathrm{E}-03$ & WS \\
\hline AQP1 & CYP1B1 & 8.99E-04 & 0.486 & $4.74 \mathrm{E}-03$ & WS \\
\hline ATF4 & COL4A1 & $4.38 \mathrm{E}-04$ & 0.490 & $4.41 \mathrm{E}-03$ & WS \\
\hline ATF4 & COL4A2 & $1.24 \mathrm{E}-04$ & 0.556 & $1.25 \mathrm{E}-03$ & WS \\
\hline ATF4 & COL15A1 & 3.70E-05 & 0.602 & $4.60 \mathrm{E}-04$ & WS \\
\hline ATF4 & HSPG2 & 4.43E-05 & 0.598 & 4.99E-04 & WS \\
\hline ATF4 & LAMA5 & $4.51 \mathrm{E}-04$ & 0.490 & 4.45E-03 & WS \\
\hline
\end{tabular}




\begin{tabular}{|c|c|c|c|c|c|}
\hline ATF4 & NOTCH1 & 5.51E-04 & 0.556 & $1.24 \mathrm{E}-03$ & WS \\
\hline ATF4 & ADAM17 & $6.03 E-04$ & 0.475 & 5.77E-03 & WS \\
\hline ATF4 & VWF & $5.48 \mathrm{E}-04$ & 0.513 & $2.91 \mathrm{E}-03$ & WS \\
\hline ATF4 & ADAM9 & $2.60 \mathrm{E}-04$ & 0.516 & $2.75 \mathrm{E}-03$ & WS \\
\hline ATF4 & ER01L & $1.48 \mathrm{E}-04$ & 0.548 & $1.48 \mathrm{E}-03$ & WS \\
\hline ATF4 & HSD17B12 & 2.81E-05 & 0.614 & $3.44 \mathrm{E}-04$ & WS \\
\hline ATF4 & COL21A1 & $9.11 \mathrm{E}-05$ & 0.616 & $3.29 E-04$ & WS \\
\hline CTSD & ITGA11 & 7.33E-04 & 0.515 & $2.82 \mathrm{E}-03$ & WS \\
\hline EDEM1 & CHI3L1 & $9.45 \mathrm{E}-04$ & 0.488 & 4.61E-03 & WS \\
\hline EGFR & ACTN1 & $7.05 E-06$ & 0.811 & $4.38 \mathrm{E}-07$ & WS \\
\hline EGFR & SMOC1 & 3.09E-04 & 0.609 & $3.89 \mathrm{E}-04$ & WS \\
\hline EIF2B3 & ITGB7 & 8.99E-04 & 0.538 & $1.78 \mathrm{E}-03$ & WS \\
\hline EIF5A & EGFLAM & $5.49 \mathrm{E}-04$ & 0.489 & $4.52 \mathrm{E}-03$ & WS \\
\hline ERRFI1 & CST3 & $1.51 \mathrm{E}-05$ & 0.654 & $1.25 \mathrm{E}-04$ & WS \\
\hline ERRFI1 & ITGB4 & 3.93E-05 & 0.621 & $2.92 \mathrm{E}-04$ & WS \\
\hline ERRFI1 & LAMA3 & 4.19E-05 & 0.614 & $3.46 \mathrm{E}-04$ & WS \\
\hline ERRFI1 & TGFB3 & 8.41E-04 & 0.488 & $4.55 \mathrm{E}-03$ & WS \\
\hline ERRFI1 & PRDX4 & $5.82 \mathrm{E}-04$ & 0.500 & $3.70 \mathrm{E}-03$ & WS \\
\hline GADD45B & COL21A1 & $9.58 \mathrm{E}-04$ & 0.522 & $2.47 \mathrm{E}-03$ & WS \\
\hline HILPDA & GFOD2 & 7.80E-04 & 0.474 & $5.88 \mathrm{E}-03$ & WS \\
\hline HSF4 & SPON2 & $5.66 \mathrm{E}-04$ & 0.590 & $5.94 \mathrm{E}-04$ & WS \\
\hline HSP90AB4P & LAMB2 & $6.13 \mathrm{E}-04$ & 0.517 & $2.71 \mathrm{E}-03$ & WS \\
\hline HSP90AB4P & COL21A1 & $6.73 \mathrm{E}-04$ & 0.517 & $2.68 \mathrm{E}-03$ & WS \\
\hline HSPB11 & COL21A1 & $6.82 \mathrm{E}-04$ & 0.593 & $5.65 E-04$ & WS \\
\hline MAPK7 & MPZL3 & $6.62 \mathrm{E}-04$ & 0.518 & $2.65 \mathrm{E}-03$ & WS \\
\hline PGK1 & GFOD2 & $3.98 \mathrm{E}-04$ & 0.710 & $2.44 \mathrm{E}-05$ & WS \\
\hline PRDX6 & $\mathrm{BCL} 3$ & $9.33 \mathrm{E}-04$ & 0.497 & $3.86 \mathrm{E}-03$ & WS \\
\hline SQSTM1 & NOTCH1 & 8.75E-04 & 0.630 & $2.34 \mathrm{E}-04$ & WS \\
\hline SQSTM1 & P4HA1 & 2.09E-04 & 0.560 & $1.15 \mathrm{E}-03$ & WS \\
\hline SQSTM1 & MPZL3 & $5.43 \mathrm{E}-04$ & 0.552 & $1.36 \mathrm{E}-03$ & WS \\
\hline STK39 & CHI3L1 & 8.63E-04 & 0.495 & $4.06 \mathrm{E}-03$ & WS \\
\hline STK39 & PDGFRA & $9.63 \mathrm{E}-04$ & 0.460 & $7.36 \mathrm{E}-03$ & WS \\
\hline TENM1 & COL21A1 & $9.84 \mathrm{E}-04$ & 0.505 & $3.37 \mathrm{E}-03$ & WS \\
\hline TPI1 & SERPINH1 & $3.42 \mathrm{E}-04$ & 0.568 & $9.62 \mathrm{E}-04$ & WS \\
\hline TPI1 & COL1A2 & $6.81 \mathrm{E}-04$ & 0.525 & $2.29 \mathrm{E}-03$ & WS \\
\hline TPI1 & FBN1 & 4.57E-04 & 0.542 & $1.66 \mathrm{E}-03$ & WS \\
\hline TPI1 & NPNT & $1.05 \mathrm{E}-04$ & 0.618 & $3.10 E-04$ & WS \\
\hline TUBB & CMA1 & $6.41 \mathrm{E}-04$ & 0.527 & $2.25 \mathrm{E}-03$ & WS \\
\hline TUBB & CCDC80 & 4.62E-04 & 0.573 & $8.65 \mathrm{E}-04$ & WS \\
\hline ACTG1 & MMP15 & $3.40 \mathrm{E}-04$ & 0.770 & $1.35 \mathrm{E}-06$ & DI \\
\hline $\mathrm{C} 5$ & ADAM10 & 2.47E-04 & 0.547 & $1.03 \mathrm{E}-03$ & DI \\
\hline C5 & DDR1 & $2.15 \mathrm{E}-04$ & 0.555 & $8.65 \mathrm{E}-04$ & DI \\
\hline C5 & CD47 & 3.07E-06 & 0.711 & $1.29 \mathrm{E}-05$ & DI \\
\hline C5 & COL6A3 & 6.37E-04 & 0.509 & $2.26 \mathrm{E}-03$ & DI \\
\hline C5 & ENG & $6.30 \mathrm{E}-04$ & 0.508 & $2.30 \mathrm{E}-03$ & DI \\
\hline C5 & ICAM1 & 8.53E-04 & 0.485 & $3.56 \mathrm{E}-03$ & DI \\
\hline C5 & ITGAD & $4.73 \mathrm{E}-04$ & 0.551 & $9.44 \mathrm{E}-04$ & DI \\
\hline C5 & PDGFRA & $8.45 \mathrm{E}-04$ & 0.535 & $1.32 \mathrm{E}-03$ & DI \\
\hline C5 & ADAM9 & 2.94E-04 & 0.602 & $2.95 \mathrm{E}-04$ & DI \\
\hline C5 & ERO1LB & 4.75E-05 & 0.630 & $1.46 \mathrm{E}-04$ & DI \\
\hline C5 & KAZALD1 & $2.49 \mathrm{E}-04$ & 0.615 & $2.13 E-04$ & DI \\
\hline CLIC1 & COL8A2 & $1.87 \mathrm{E}-04$ & 0.549 & $9.84 \mathrm{E}-04$ & DI \\
\hline CLIC1 & COL16A1 & 4.64E-04 & 0.508 & $2.27 \mathrm{E}-03$ & DI \\
\hline CLIC1 & ELN & 6.97E-04 & 0.489 & $3.30 \mathrm{E}-03$ & DI \\
\hline CLIC1 & CILP & $9.28 \mathrm{E}-04$ & 0.521 & 1.77E-03 & DI \\
\hline CLIC1 & NID2 & $3.24 \mathrm{E}-04$ & 0.526 & $1.60 \mathrm{E}-03$ & DI \\
\hline DUSP10 & ELN & 5.14E-04 & 0.562 & $7.48 \mathrm{E}-04$ & DI \\
\hline EDEM1 & CD151 & $5.63 \mathrm{E}-04$ & 0.686 & $5.06 \mathrm{E}-05$ & DI \\
\hline HEY2 & VWF & $1.35 \mathrm{E}-04$ & 0.537 & $1.29 \mathrm{E}-03$ & DI \\
\hline MAPK9 & ERO1L & $3.43 \mathrm{E}-04$ & 0.488 & $3.34 \mathrm{E}-03$ & DI \\
\hline MEF2C & ITGAD & $2.75 \mathrm{E}-04$ & 0.604 & 2.79E-04 & DI \\
\hline PKM & CILP & 5.15E-04 & 0.578 & $5.16 \mathrm{E}-04$ & DI \\
\hline PKM & ADAMTS4 & 8.23E-04 & 0.522 & $1.74 \mathrm{E}-03$ & DI \\
\hline PRDX6 & TIMP4 & 7.96E-04 & 0.607 & $2.58 \mathrm{E}-04$ & DI \\
\hline RGS14 & CSGALNACT1 & $6.03 E-04$ & 0.562 & $7.48 \mathrm{E}-04$ & DI \\
\hline RPS6KA3 & CD47 & 4.65E-04 & 0.470 & $4.62 \mathrm{E}-03$ & DI \\
\hline RPS6KA3 & MADCAM1 & $2.42 \mathrm{E}-04$ & 0.501 & $2.63 \mathrm{E}-03$ & DI \\
\hline RPS6KA3 & COL5A3 & $3.23 \mathrm{E}-05$ & 0.591 & $3.85 \mathrm{E}-04$ & DI \\
\hline RPS6KA3 & CTRB2 & $8.48 \mathrm{E}-04$ & 0.448 & $6.87 \mathrm{E}-03$ & DI \\
\hline RTN3 & TIMP4 & $3.53 \mathrm{E}-04$ & 0.611 & $2.33 \mathrm{E}-04$ & DI \\
\hline TPI1 & P4HA1 & $9.36 \mathrm{E}-04$ & 0.486 & $3.47 \mathrm{E}-03$ & DI \\
\hline
\end{tabular}


Chapter 6

$\begin{array}{llllll}\text { TUBB } & \text { FAP } & 5.91 \mathrm{E}-04 & 0.528 & 1.55 \mathrm{E}-03 & \text { DI } \\ \text { TUBB } & \text { TGFB2 } & 4.53 \mathrm{E}-04 & 0.521 & 1.76 \mathrm{E}-03 & \text { DI }\end{array}$

Interactions $(P \leq 0.001)$ between stress- and ECM-related genes. $r^{2}(\geq 0.500)$ and Model P-value $(\leq 0.01)$ depict the effect of the interaction on the weight regain percentage. WL, weight loss; WS, weight stable; DI, dietary intervention. 


\section{Chapter 7}

General discussion 
Over the years it has become increasingly clear that overweight and obesity is a serious health problem. Weight loss (WL) reduces disease risk and improves the metabolic profile resulting in positive health outcomes $(1,2)$. Weight loss can be readily achieved and the most common approach to losing weight is dieting, however, long-term weight reduction is proven to be difficult $(3,4)$. Most people eventually regain the lost weight. Weight regain is one of the most significant obstacles in obesity management, therefore, it is of great importance to understand the underlying mechanism behind weight regain. The involvement of environmental and behavioural factors in the process of weight regain has been established, however, there is now substantial evidence that biological and metabolic mechanisms also contribute to the risk for weight regain (5-7). It was proposed that during a negative energy balance cellular stress arises in adipocytes due to a misfit between cell volume and the surrounding ECM. This stress can be resolved by remodeling of the ECM or by the re-storing of triglycerides within the adipocytes (8). It suggests that cellular stress accumulating in adipocytes during a negative energy balance is a driving force behind the risk for weight regain. For this thesis, studies have been conducted to better understand the underlying mechanisms of weight regain. Specifically, the relation between weight regain and adipocyte ECM- related (chapters 2 and 3) and stress-related (chapter 4 and 5) factors was investigated. In both chapter 3 and 5, we show the importance of a weight stabilisation (WS) period for the risk of weight regain and this will be discussed in the paragraph: "Weight regain: a matter of negative energy balance versus energy balance". In chapter 2, we observed that genetic variation of ECM-related genes is associated with variation in risk for weight regain. Also, we found that changes in expression of ECM-related genes correlated with weight regain (chapter 3 ). Therefore, the role of the ECM in weight management will be discussed in the paragraph entitled "Extracellular matrix genetics involved in weight regain". Furthermore, we observed that resident inflammation in the adipose tissue after weight loss might increase the risk for weight regain (chapter 3 ). The relation between inflammation and weight regain will be discussed in the paragraph entitled "Weight regain and inflammation". In chapter 4, we report about the higher cellular stress in the subcutaneous adipose tissue of participants regaining lost weight during the follow-up period compared to the participants maintaining weight loss. Furthermore, we show that the risk for weight regain is related to changes in the expression of stress-related genes during the first four weeks after returning to a weight stable situation (chapter 5). The involvement of stress in weight management will be discussed in the paragraph entitled "Weight regain: a stressful matter". Interestingly, we saw that the adipocyte size increases during the WS period independent of changes in body weight of the participant which will be further discussed in the paragraph "Adipocyte size increases during WS".

\section{Weight regain: a matter of negative energy balance versus energy balance}

Gene expression is differentially regulated during the calorie restriction phase of a dietary weight loss program. Gene expression changes during calorie restriction represent the effect of WL plus that of a negative energy balance. A negative energy balance cannot be maintained indefinitely, therefore, gene expression patterns should also be studied during energy balance by introducing a weight stabilization phase. Previous studies have shown that gene expression is differentially regulated during the various phases of a dietary weight loss program $(9,10)$, which should be viewed as biologically distinct. Therefore, determining predictive genes for weight regain should be done during three phases: first, the WL phase which represents the effect of WL plus that of a negative energy balance; second, the WS phase which represents WL without a negative energy balance by 
comparing values after WL to values after WS; third, the dietary intervention (DI) phase which is the WL and WS phase together. This phase also represents WL without a negative energy balance and here comparisons are made between study start and after WS. The WL phase in relation to weight regain has been extensively studied. Mutch et al. showed that gene sets related to apoptosis, citric acid cycle, fatty acid metabolism and oxidative phosphorylation are regulated differently for participants regaining weight compared to participants maintaining the lower body weight (11). Another study showed that gene sets related to cellular growth and proliferation, cell death and cellular function are the main biological processes related to regain of weight (12). However, there are fewer studies about weight regain that take into account the effects of the negative energy balance on gene expression results. In our analyses, we observed that correlations between gene expression changes and weight regain are mainly found during the WS phase and not during the WL phase or the DI phase (chapter 3 and 5). These results clearly indicated that the various phases of a dietary weight loss program should be viewed separately, which is in agreement with previous studies $(9,10)$, and that for weight regain studies the changes during WS are most relevant, i.e. the return from an negative energy balance and not the weight loss per se. Perhaps this is due to the fact that molecular processes during a transition from negative to balanced energy state (WS) are in line with processes following transition from a balanced state to a positive energy balance (WR). To our knowledge there are no other human dietary intervention studies focussed on weight regain that include a WS phase.

Capel et al. used a study design with a VLCD period followed by a LCD phase, then followed by three months weight maintenance (9). They showed distinct patterns of gene regulation during the various phases of a dietary weight loss program, however, they did not relate these changes to weight regain. Future dietary intervention studies, especially studies related to prediction of weight regain, should contain a WS phase to get rid of the influence of the negative energy balance.

\section{Extracellular matrix genetics involved in weight regain}

Weight loss becomes more difficult after several weeks in a negative energy balance because other components involved in maintenance of the energy balance will be changed, i.e. the energy expenditure and body energy stores are decreased $(13,14)$ while hunger is increased $(15)$. For many people it is difficult to sustain weight loss (8) and the question remains what lies at the basis of this difficulty. It has become increasingly clear that the adipose tissue plays an important role in the risk for weight regain. Adipocytes spend a lot of their energy on development, renewal and maintenance of the ECM (16), which is known for providing structural support to the adipocyte. A large part of the energy is used for the rapid turnover of collagens, whereas the turnover of proteins from the cytoskeleton is considerably slower (16). Clearly, there is a role for the ECM in adipocyte biology with reflection on weight regulation. It was shown that ECM regulated processes are disturbed in the obese $(17,18)$, that ECM-regulating genes are differentially expressed in adipose tissue after WL $(19$, 20) and that weight gain leads to different expression patterns of ECM-remodelling genes (21). However, the role of the ECM in the process of weight regain has been less studied. When comparing gene expression after WL between participants regaining only $0-10 \%$ (weight maintainers) and those who regained $50-100 \%$ (weight regainers) of the lost weight it was seen that genes of the focal adhesion pathway, important in the coordination of the ECM, were down-regulated in weight maintainers while up-regulated in weight regainers (11). In our studies, we saw that genetic variations of ECM-related genes are associated with an increased risk for weight regain. Specifically, 
we saw the involvement of variants of the POSTN, LAMB1, COL23A1, and FBLN5 genes for males and of the FN1 gene for females (chapter 2). Indeed, some of those genes point as well to a role for focal adhesions, i.e. the sites where the ECM is attached to the cytoskeletal actin fibres. During WL, energy stores are mobilized from adipocytes causing a decrease in adipocyte size, which is reversed when sufficient energy is available (22-25). The adipocyte diameter decreases with about $10 \%$ during WL (Vink RG, Roumans NJ et al., submitted). As adipocytes change size the ECM must be remodelled to accommodate the change or a considerable mechanical strain will be imposed upon the adipocytes (26). In accord with our findings and those of others, it can be expected that mechanical strain will concentrate on the ECM-attachment sites, i.e. the focal adhesions. These genes may therefore be of interest for future genetic research on weight regulation and obesity.

Besides genes mediating stress at the focal adhesions, also genes for ECM remodelling can be expected to influence the risk for weight regain. Remodelling of the ECM involves the turnover of ECM proteins like collagens. For the construction of new collagen fibres, collagen proteins have to be synthesized and modified, for instance by prolyl-hydroxylase, which is an energy-demanding process directed by insulin $(27,28)$. However, under conditions of calorie restriction during $W L$, such energy is not available and insulin levels are low, but when returning to energy balance (WS), such genes may become apparent in gene expression measurements related to weight regain. As described in the paragraph "Weight regain: a matter of negative energy balance versus energy balance", we observed that changes in expression of ECM-modifying genes are related to weight regain mainly during the WS phase (chapter 3). Examples of such genes are COLGALT1, PLOD1 and P4HB. Together, our studies show that the ECM plays a role in the risk for weight regain and suggest that two processes dominate this risk: accommodation of stress at the focal adhesions and ECM remodelling to reduce ECM misfit and cellular stress.

\section{Weight regain and inflammation}

The development of obesity is accompanied by the onset of low-grade inflammation. This inflammation is caused by the dysregulated production and release of cytokines and adipokines, such as interleukin-6, tumor necrosis factor and monocyte chemoattractant protein-1. Cells of the innate immune system are attracted into the adipose tissue leading to a situation in which proinflammatory immune cells outbalance the anti-inflammatory cells (29). This increased inflammation may play a role in the pathogenesis of insulin resistance and cardiovascular disease (30). Weight loss usually reduces the inflammatory activity of the adipose tissue, which may not be directly obvious during energy restriction. Studies have shown that the expression of genes involved in inflammation and innate immunity was upregulated $(9,31)$ or unchanged $(10)$ in human adipose tissue directly after short-term weight loss. We observed that expression of these genes was downregulated during the subsequent WS period (31). Considering that whole body inflammation can be dictated by loss of body weight, it is reasonable to suggest that inflammation could influence regain of weight (32). Large weight gain occurs more frequently in participants with elevated levels of four putative markers of inflammation (33). Also, it has been shown that participants with a more advanced proinflammatory state at the end of a dietary intervention are more prone to regain weight (34). We observed correlations between expression changes of leukocyte-specific integrins during WS and the weight regain percentage (chapter 3 ). Those genes are down-regulated during WS and are clustered with genes for ECM remodelling. It is therefore tempting to speculate, that emigration of leukocytes after weight loss reduces the risk for weight regain and that failure to accomplish this by hampered 
ECM remodeling leads more often to weight regain. Although we have not investigated this in detail here, our results point to an interaction between the change of inflammatory status during weight loss and shortly after, and weight regain.

\section{Weight regain: a stressful matter}

After WL and returning to energy balance, the expression patterns of proteins belonging to the stress proteome are changed (35-37). The easiest way for adipocytes to get rid of this cellular stress is by returning to their original volume by re-storing fat (38). For the host this would mean that after WL there is increased risk for weight regain originating from the cellular stress of the adipocytes. In line, we observed higher levels of stress-related factors after WL for participants regaining weight (chapter 4). As shown, in parallel with changes in proteins of the stress proteome adipocytes prepare for renewed storage of fat (35). Bouwman et al. showed that the translocation of GLUT4 is enhanced after returning to energy balance resulting in increased capacity for the uptake of glucose (35). In addition, a specific role was attributed to aldolase as an anchoring protein for GLUT4-vesicles onto the actin filaments. Similar to the ECM-related genes we saw that changes in expression of stressrelated genes during WL are less related to weight regain while the opposite is observed for the WS phase (chapter 5). Accordingly, we showed that the stress-related genes correlating with weight regain during WS are involved in actin filament dynamics, glucose handling and nutrient sensing (chapter 5). It is tempting to speculate that cellular stress accumulating in the adipocytes during weight loss and during weight stabilisation, induces metabolic changes towards increased uptake of energy, which would increase the risk for weight regain by the host.

Another way in which cellular stress might increase food intake and weight regain would be by the secretion of signals known as adipokines which can affect appetite, satiety and energy expenditure $(39,40)$. The secretion pattern of adipokines is related to the size of an adipocyte $(41)$.Thus WL, which leads to a decreased adipocyte size, will also lead to a different adipokine secretion profile. These changes in secretion profile could increase food intake by stimulating the hunger feeling leading to the increased uptake and storage of energy. The satiety hormone leptin is a classic example of an adipokine that decreases dramatically during weight loss. In our studies, a relation between weight regain and adipokines after returning to energy balance has been established (Vink RG, Roumans NJ et al., submitted). However, we have not been able to link the changes in stress gene expression to changes in secretion of adipokines. Additional research is needed to test this. Also, we were able to measure the levels of 10 adipokines only, whereas a broader adipokine profilebased approach might provide more insight in the possible involvement of stress-induced adipokines in weight regain.

Both stress- and ECM-related factors are independently associated with the risk for weight regain, but we also saw that the combination of stress- and ECM-related factors can have a larger effect on weight regain. As shown in chapter 6, the stress gene EGFR is an important node in the risk for weight regain. The interaction between this stress gene with the ECM gene ACTN1 during the WS phase has the strongest effect on the weight regain percentage. Furthermore, ERRFI1, which is a negative regulator of EGFR, has interactions with the stress genes CST3, ITGB4 and LAMA3. All these interactions had a strong effect on the weight regain percentage. This indicates that the process of weight regain is complex and multiple factors are involved in the underlying process. 


\section{Adipocyte size increases during WS}

It can be expected that adipocyte volume decreases during WL and that this may lead to cellular stress due to the misfit between cell volume and the surrounding ECM. This stress can be resolved by adjustment of the surrounding ECM or by re-storing fat to return to the original volume (8). The restoring of fat would lead to regain of lost weight by the host. This suggests that besides ECM and stress, also changes in adipocyte size could mark the risk for weight regain and it is hypothesized that the change in adipocyte size is related to change in body weight. In our study, we also measured adipocyte size and as expected, the diameter decreased with loss of body weight (Figure 1).

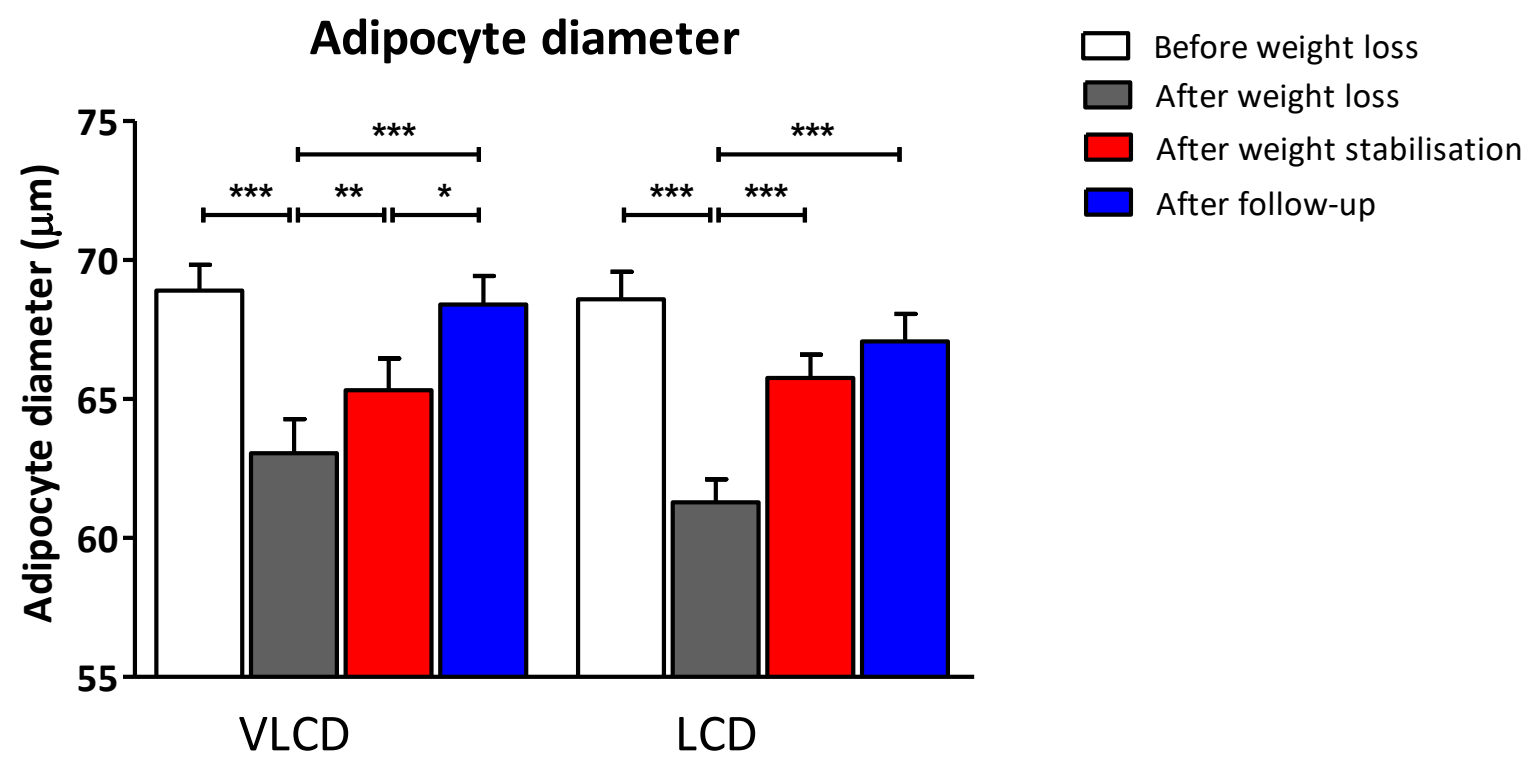

Figure 1: Adipocyte size measured on $8 \mu \mathrm{m}$ paraffin-embedded sections of adipose tissue stained with hematoxylin and eosin. ${ }^{*} \mathrm{P} \leq 0.05,{ }^{* *} \mathrm{P}<0.01,{ }^{* * *} \mathrm{P}<0.001$ with dependent $\mathrm{T}$-test to observed within-group change of adipocyte size.

The adipocyte size was expected not to change during the WS phase because then the diet of each participant is based on the energy requirements of each individual, which is confirmed by the stable body weight of the participants. However, to our surprise, adipocyte size was observed to increase considerably in the WS period for all participants. Based on our model for stress-related energyuptake, this suggests a strong influx of energy into the adipocytes from the time when the cellular stress is highest, i.e. immediately after WL. The question is, where this energy comes from. We know that adipocytes prepare for glucose uptake and we observed that during WS the gene coding for the enzyme fatty acid synthase, responsible for de novo adipogenesis, is upregulated. However, the diet during WS would not provide enough energy for the adipocytes to refill because this diet is based on the energy requirement of each participant to assure weight maintenance. More likely, fat from other parts of the body, such as the visceral adipose tissue or liver or muscle, is used to refill the stressed adipocytes in the subcutaneous adipose tissue. In this respect, this phenomenon would represent an improvement of metabolic health by moving fat to where it belongs, i.e. the adipose tissue. It should be noted, however, that we have no proof that the increase in adipocyte size is due to the influx of energy. It may well be caused by something else like accumulation of water, or may even be an artifact of tissue preparation due to a more flexible ECM after WS. Additional experiments need to be undertaken to provide clarity in this matter. 
Change in adipocyte diameter during WL, WS or DI was not correlated to the weight regain percentage or the change in body weight during follow-up. The same was true for the calculated and corrected adipocyte volume (42). Rapid re-storage of fat would be contradictory to our model for stress-induced weight regain, because it would rapidly diminish the biological drive. However, as mentioned, this process needs further investigation. Anyway, adipocyte size cannot be used as a predictor of weight regain.

\section{Conclusions}

This thesis focussed on gaining insight in the process of weight regain after weight loss to determine possible predictors for weight regain. This insight can be used for better guidance in weight control. The major conclusions of this thesis are the following:

- Dietary intervention studies should contain a weight stabilisation phase to monitor the return to energy balance especially when gene expression results are used to determine predictive targets of weight regain.

- Changes in expression of stress- and ECM-related genes including the interaction of these changes are highly related to the weight regain percentage.

- Epidermal growth factor activity strongly influences the correlation of stress and ECM genes with weight regain percentage.

- People who regained most of their weight during follow-up have increased cellular stress levels after an 8-week energy restricted diet.

- The risk for weight regain is related to expression changes of distinct sets of stress-related genes during the first four weeks after returning to energy balance. These genes are involved in actin filament dynamics, glucose handling and nutrient sensing.

- Polymorphisms of the ECM genes COL23A1, FBLN5, LAMB1 and POSTN in men and FN1 in women are associated with weight regain after weight loss pointing to a role for focal adhesions in the risk for weight regain.

- People with a stronger downregulation of ECM-remodelling may retain more (active) immune cells and have higher risk of weight regain suggesting that resident inflammation after weight loss increases the risk for weight regain.

- Changes in adipocyte diameter are not correlated with changes of body weight after weight loss.

\section{Suggestions for future research}

Gene expression is differentially regulated during the calorie restriction phase and the weight stabilisation phase of a dietary weight loss program. Correlations between gene expression changes and weight regain are mainly found during the weight stabilisation phase. Therefore, future human dietary intervention studies focussed on predictive gene targets of weight regain should contain a weight stabilisation period. However, the length of the weight stabilisation period for the effects of a negative energy balance to have worn out needs to be determined.

Subcutaneous adipose tissue samples taken were used for gene expression analyses. These biopsies contain adipocytes as well as stromal vascular cells, which can obscure the exact contribution of each 
cell type in the tissue to the biological processes involved here. Therefore, it would be of great interest to investigate separately purified adipose tissue cell fractions.

The adipocyte diameter increases during the WS phase while the body weight of the participants remained stable. It suggests that fat from other parts of the body is used to refill the adipocyte thereby reducing the stressed created by the misfit between the ECM and the lower adipocyte volume. However, our study has not looked into changes in other fat depots. Further analyses are necessary to confirm these novel findings and unravel the underlying process. For this, the adipocyte size should be monitored in both subcutaneous and visceral adipose tissue during weight loss and a subsequent weight stabilisation period. Reallocation of fat might be assessed by repeated DEXAscans during the intervention.

Our analyses on ECM-related genes showed that resident inflammation after weight loss may increase the risk for weight regain. However, this was found in a gene-set which was not focussed on inflammation. Therefore, it would be interesting to focus on the relation between inflammation or the inflammatory status of the adipose tissue and the risk for weight regain after weight loss. 


\section{References}

1. Horton ES. Effects of lifestyle changes to reduce risks of diabetes and associated cardiovascular risks: results from large scale efficacy trials. Obesity (Silver Spring), 2009. 17 Suppl 3:S43-8.

2. Van Gaal LF, Wauters MA, De Leeuw IH. The beneficial effects of modest weight loss on cardiovascular risk factors. Int J Obes Relat Metab Disord, 1997. 21 Suppl 1:S5-9.

3. Barte JC, ter Bogt NC, Bogers RP, Teixeira PJ, Blissmer B, Mori TA, Bemelmans WJ. Maintenance of weight loss after lifestyle interventions for overweight and obesity, a systematic review. Obes Rev, 2010. 11(12):899-906.

4. Wu T, Gao X, Chen M, van Dam RM. Long-term effectiveness of diet-plus-exercise interventions vs. diet-only interventions for weight loss: a meta-analysis. Obes Rev, 2009. 10(3):313-23.

5. Maclean PS, Bergouignan A, Cornier MA, Jackman MR. Biology's response to dieting: the impetus for weight regain. Am J Physiol Regul Integr Comp Physiol, 2011. 301(3):R581-600.

6. MacLean PS, Higgins JA, Giles ED, Sherk VD, Jackman MR. The role for adipose tissue in weight regain after weight loss. Obes Rev, 2015. 16 Suppl 1:45-54.

7. Mariman EC. Human biology of weight maintenance after weight loss. J Nutrigenet Nutrigenomics, 2012. 5(1):13-25.

8. Mariman EC. An adipobiological model for weight regain after weight loss. Adipobiology, 2011. 3(1):915.

9. Capel F, Klimcakova E, Viguerie N, Roussel B, Vitkova M, Kovacikova M et al. Macrophages and adipocytes in human obesity: adipose tissue gene expression and insulin sensitivity during calorie restriction and weight stabilization. Diabetes, 2009. 58(7):1558-67.

10. Johansson LE, Danielsson AP, Parikh H, Klintenberg M, Norstrom F, Groop L, Ridderstrale $M$. Differential gene expression in adipose tissue from obese human subjects during weight loss and weight maintenance. The American journal of clinical nutrition, 2012. 96(1):196-207.

11. Mutch DM, Pers TH, Temanni MR, Pelloux V, Marquez-Quinones A, Holst C et al. A distinct adipose tissue gene expression response to caloric restriction predicts 6-mo weight maintenance in obese subjects. Am J Clin Nutr, 2011. 94(6):1399-409.

12. Marquez-Quinones A, Mutch DM, Debard C, Wang P, Combes M, Roussel B et al. Adipose tissue transcriptome reflects variations between subjects with continued weight loss and subjects regaining weight 6 mo after caloric restriction independent of energy intake. The American journal of clinical nutrition, 2010. 92(4):975-84.

13. Dulloo AG, Jacquet J. Adaptive reduction in basal metabolic rate in response to food deprivation in humans: a role for feedback signals from fat stores. The American journal of clinical nutrition, 1998. 68(3):599-606.

14. Jebb SA, Prentice AM, Goldberg GR, Murgatroyd PR, Black AE, Coward WA. Changes in macronutrient balance during over- and underfeeding assessed by 12-d continuous whole-body calorimetry. The American journal of clinical nutrition, 1996. 64(3):259-66.

15. Schwartz MW, Woods SC, Porte D, Jr., Seeley RJ, Baskin DG. Central nervous system control of food intake. Nature, 2000. 404(6778):661-71.

16. Bouwman F, Renes J, Mariman E. A combination of protein profiling and isotopomer analysis using matrix-assisted laser desorption/ionization-time of flight mass spectrometry reveals an active metabolism of the extracellular matrix of 3T3-L1 adipocytes. Proteomics, 2004. 4(12):3855-63.

17. Divoux A, Tordjman J, Lacasa D, Veyrie N, Hugol D, Aissat A et al. Fibrosis in human adipose tissue: composition, distribution, and link with lipid metabolism and fat mass loss. Diabetes, 2010. 59(11):2817-25.

18. Strissel KJ, Stancheva Z, Miyoshi H, Perfield JW, 2nd, DeFuria J, Jick Z, Greenberg AS, Obin MS. Adipocyte death, adipose tissue remodeling, and obesity complications. Diabetes, 2007. 56(12):29108.

19. Henegar C, Tordjman J, Achard V, Lacasa D, Cremer I, Guerre-Millo M et al. Adipose tissue transcriptomic signature highlights the pathological relevance of extracellular matrix in human obesity. Genome Biol, 2008. 9(1):R14.

20. Kolehmainen M, Salopuro T, Schwab US, Kekalainen J, Kallio P, Laaksonen DE et al. Weight reduction modulates expression of genes involved in extracellular matrix and cell death: the GENOBIN study. Int J Obes (Lond), 2008. 32(2):292-303. 
21. Tam CS, Covington JD, Bajpeyi S, Tchoukalova Y, Burk D, Johannsen DL, Zingaretti CM, Cinti S, Ravussin E. Weight gain reveals dramatic increases in skeletal muscle extracellular matrix remodeling. J Clin Endocrinol Metab, 2014. 99(5):1749-57.

22. Gurr MI, Jung RT, Robinson MP, James WP. Adipose tissue cellularity in man: the relationship between fat cell size and number, the mass and distribution of body fat and the history of weight gain and loss. International journal of obesity, 1982. 6(5):419-36.

23. Miller WH, Jr., Faust IM, Goldberger AC, Hirsch J. Effects of severe long-term food deprivation and refeeding on adipose tissue cells in the rat. The American journal of physiology, 1983. 245(1):E74-80.

24. Portillo MP, Cantoral R, Macarulla MT. Effects of dietary fat content on adiposity during energy restriction in genetically obese rats. Reproduction, nutrition, development, 1999. 39(2):189-99.

25. MacLean PS, Higgins JA, Jackman MR, Johnson GC, Fleming-Elder BK, Wyatt HR, Melanson EL, Hill JO. Peripheral metabolic responses to prolonged weight reduction that promote rapid, efficient regain in obesity-prone rats. American journal of physiology Regulatory, integrative and comparative physiology, 2006. 290(6):R1577-88.

26. Lee MJ, Wu Y, Fried SK. Adipose tissue remodeling in pathophysiology of obesity. Current opinion in clinical nutrition and metabolic care, 2010. 13(4):371-6.

27. Mariman EC, Wang P. Adipocyte extracellular matrix composition, dynamics and role in obesity. Cell Mol Life Sci, 2010. 67(8):1277-92.

28. Wang P, Keijer J, Bunschoten A, Bouwman F, Renes J, Mariman E. Insulin modulates the secretion of proteins from mature 3T3-L1 adipocytes: a role for transcriptional regulation of processing. Diabetologia, 2006. 49(10):2453-62.

29. Choe SS, Huh JY, Hwang IJ, Kim JI, Kim JB. Adipose Tissue Remodeling: Its Role in Energy Metabolism and Metabolic Disorders. Frontiers in endocrinology, 2016. 7:30.

30. Gruson E, Montaye M, Kee F, Wagner A, Bingham A, Ruidavets JB et al. Anthropometric assessment of abdominal obesity and coronary heart disease risk in men: the PRIME study. Heart, 2010. 96(2):13640.

31. Vink RG, Roumans NJ, Fazelzadeh P, Tareen SH, Boekschoten MV, van Baak MA, Mariman EC. Adipose tissue gene expression is differentially regulated with different rates of weight loss in overweight and obese humans. International journal of obesity, 2016.

32. Das UN. Is obesity an inflammatory condition? Nutrition, 2001. 17(11-12):953-66.

33. Duncan BB, Schmidt MI, Chambless LE, Folsom AR, Carpenter M, Heiss G. Fibrinogen, other putative markers of inflammation, and weight gain in middle-aged adults--the ARIC study. Atherosclerosis Risk in Communities. Obesity research, 2000. 8(4):279-86.

34. Goyenechea E, Parra D, Crujeiras AB, Abete I, Martinez JA. A nutrigenomic inflammation-related PBMC-based approach to predict the weight-loss regain in obese subjects. Annals of nutrition \& metabolism, 2009. 54(1):43-51.

35. Bouwman FG, Claessens M, van Baak MA, Noben JP, Wang P, Saris WH, Mariman EC. The physiologic effects of caloric restriction are reflected in the in vivo adipocyte-enriched proteome of overweight/obese subjects. J Proteome Res, 2009. 8(12):5532-40.

36. Wang P, Bouwman FG, Mariman EC. Generally detected proteins in comparative proteomics--a matter of cellular stress response? Proteomics, 2009. 9(11):2955-66.

37. Mariman EC. 2DE-proteomics meta-data indicate the existence of distinct cellular stress-responsive mechanisms. Expert review of proteomics, 2009. 6(4):337-9.

38. Eastman Q. Very low calorie diet makes adipocytes "scream". Journal of proteome research, 2009. 8(12):5408.

39. Bluher M. Adipokines - removing road blocks to obesity and diabetes therapy. Molecular metabolism, 2014. 3(3):230-40.

40. Kloting N, Kovacs P, Kern M, Heiker JT, Fasshauer M, Schon MR, Stumvoll M, Beck-Sickinger AG, Bluher $M$. Central vaspin administration acutely reduces food intake and has sustained blood glucoselowering effects. Diabetologia, 2011. 54(7):1819-23.

41. Skurk T, Alberti-Huber C, Herder C, Hauner H. Relationship between adipocyte size and adipokine expression and secretion. The Journal of clinical endocrinology and metabolism, 2007. 92(3):1023-33.

42. Lenz M, Roumans NJ, Vink RG, van Baak MA, Mariman EC, Arts IC, de Kok TM, Ertaylan G. Estimating real cell size distribution from cross-section microscopy imaging. Bioinformatics, 2016. 32(17):i396i404. 


\section{Addendum}

Summary 
Over the last decades, the prevalence of obesity has reached epidemic proportions. In 2014, the World Health Organization estimated that of adults aged 18 and over, $39 \%$ were overweight and $13 \%$ were obese. The increasing prevalence of obesity is a major health concern since it increases the risk for developing type 2 diabetes mellitus, cardiovascular diseases and certain types of cancer. The simple remedy to obesity is losing weight by limiting energy/food intake and increasing daily physical activity for a longer period of time. However, the greatest challenge is the seemingly inevitable weight regain after weight loss (WL), the so-called "yoyo-effect". In general up to $80 \%$ of the people are unsuccessful in maintaining weight loss. It has become clear that the adipose tissue plays an important role in the increased risk for weight regain after weight loss. During a negative energy balance, loss of triglycerides decreases the volume of an adipocyte. This decrease in volume creates cellular stress in adipocytes due to the misfit between cell volume and the surrounding extracellular matrix (ECM). This stress can be resolved by remodeling of the ECM or re-storing of triglycerides within the adipocytes. Re-storing of triglycerides would lead to an increase in body weight of the host. This suggests that cellular stress accumulated in adipocytes during a negative energy balance, due to the misfit between cell volume and ECM, is a driving force behind the risk for weight regain. Therefore, studies described in this thesis investigated the involvement of adipocyte stress- and ECM-related factors in the process of weight regain after weight loss.

First, we looked at the relationship between ECM-related factors and the risk for weight regain in two independent cohorts.

In Chapter 2, we looked at the role of genetic variation in 124 ECM genes in the risk of weight regain amongst participants of a Pan-European, randomized, controlled dietary intervention study (the socalled "DiOGenes study"). In this study, overweight and obese subjects (310 women, 159 males) followed an 8-week low calorie diet (LCD) with a 6-month follow-up period. Body weight was measured before and after the diet, and after follow-up. As a value for weight regain, the weight maintenance scores (WMS) were calculated as follows: (weight after follow-up - weight after WL) $\div$ (weight before $\mathrm{WL}$ - weight after $\mathrm{WL}$ ). A score equal or lower than zero indicated that the participant maintained or continued to lose weight during the follow-up period, while a score higher than zero indicated that the participant regained weight during the 6-month follow-up. Furthermore, we retrieved data for 2903 genetic variations in and near the $124 \mathrm{ECM}$-related genes. With regression analyses we determined the relation between the WMS and the genetic variations. In men, variants of the POSTN, LAMB1, COL23A1, and FBLN5 genes are related to the risk of weight regain after weight loss. In women, a variant of the FN1 gene is related to the risk of weight regain.

Chapter $\mathbf{3}$ focusses on the relation between weight regain and changes in expression of ECM genes. This was analysed in a randomized, controlled dietary intervention study (the so-called "yoyo-study") in which 61 participants lost weight by either a 5-week VLCD or a 12-week LCD, with a subsequent 4week weight stable (WS) diet, and a 9-month follow-up period. The weight loss (WL) and WS phase taken together was named the dietary intervention (DI). As a value for weight regain, the weight regain percentage (WR\%) was calculated as follows: ((weight after follow-up - weight after WS) $\div$ weight after WS) $\times 100 \%$. Next, the expression changes of 277 ECM-related genes during WL, WS and DI were correlated with the WR\%. We observed 25 genes that were strongly correlated with the WR\% and the highest number of correlations was observed in the VLCD WS phase. Five of these genes appeared to belong to a group of 26 genes of which the expression changes correlated highly among each other. This group could be divided into three clusters, one of which is mainly composed of leukocyte-specific integrin genes. Our results suggest that a lower reduction of the expression of 
certain leukocyte integrin genes shortly after weight loss by calorie restriction leads to a higher risk of weight regain, which seems linked to ECM remodelling. Possibly, a stronger reduction of ECM remodelling capacity during the WS phase leads to more retention of immune cells suggesting that resident inflammation after weight loss increases the risk for weight regain.

Second, we looked at the relationship between stress-related factors and the risk for weight regain in two independent cohorts.

In Chapter 4, we determined stress protein levels during weight loss and weight maintenance in relation to weight regain. For this, 18 healthy overweight and obese subjects underwent an 8-week VLCD with a 10-month follow-up. Participants were categorised as weight maintainers if there was a weight reduction of at least $10 \%$ but then regained less than $6 \%$ weight during follow-up. Participants were categorised as weight regainers (WR) if there was a weight reduction of at least $10 \%$ but then regained $6 \%$ or more weight. At the end of WL, WR had higher levels of the stress proteins $\beta$-actin, calnexin, heat shock protein (HSP) 27, HSP60 and HSP70. Changes of $\beta$-actin, HSP27 and HSP70 were correlated to HSP60, a proposed key factor in weight regain after weight loss. These findings underscore that adipocyte stress plays a role as a biological risk factor for weight regain.

Chapter 5 provides information about the relation between weight regain and expression changes of stress-related genes during WL, WS and DI which was investigated in the yoyo-study. Expression changes of 107 stress-related genes during WL, WS and DI were correlated with the WR\%. We found that correlations between expression changes and weight regain were more frequent for the VLCD group than for the LCD group and most correlations appeared in the WS and DI phases. Eight of the nine correlating stress genes of the WS phase were clustered. These genes indicate that there is a link between weight regain and the biological processes on actin filament dynamics, glucose handling and nutrient sensing.

Third, we looked at the relationship between stress- and ECM-related factors and their influence on the risk for weight regain in the yoyo-study.

In Chapter 6, we performed correlation and interaction analysis with the WR\% using a stress-related and an ECM-related gene set. Correlation analyses revealed that during the WS phase in the VLCD group a co-expression network could be constructed consisting of 8 stress- and 15 ECM-related genes, which all correlated with the WR\%. The network links to the biological processes on leukocyte-activity, ECM remodelling, actin cytoskeleton organisation and glucose handling. Interaction analysis between stress- and ECM-related genes revealed several gene combinations that were highly related to the weight regain percentage. In particular, the epidermal growth factor signaling pathway was identified as strongly influencing the risk of weight regain, possibly through interaction with actinin alpha-1 (ACTN1), a component of stress fibers, and with integrin beta 4 (ITGB4), cystatin C (CST3) and laminin alpha-3 (LAMA3).

This thesis provides important insights into the underlying mechanism of weight regain after weight loss. We showed that the risk for weight regain is related to expression changes of distinct sets of stress- and ECM-related genes including the interaction of these changes during the first four weeks after returning to energy balance. Furthermore, we showed that genetic variations of ECM genes are associated with weight regain. 
Samenvatting 
De prevalentie van obesitas is de afgelopen jaren enorm toegenomen. In 2014 rapporteerde de Wereldgezondheidsorganisatie dat $39 \%$ van alle volwassenen overgewicht heeft en dat $13 \%$ obese is. De toenemende prevalentie van obesitas is een groot gezondheidsprobleem omdat het leidt tot een verhoogd risico op het ontwikkelen van type 2 diabetes mellitus, cardiovasculaire aandoeningen en bepaalde vormen van kanker. De eenvoudigste oplossing om obesitas tegen te gaan is door het verlagen van het lichaamsgewicht door gedurende een langere periode de energie/voedsel inname te verlagen en de dagelijkse lichamelijke activiteit te verhogen. Gewichtsverlies kan op deze manier eenvoudig worden bereikt maar de grootste uitdaging blijft de schijnbaar onvermijdelijke gewichtstoename na gewichtsverlies, het zogenaamde "jojo-effect". Studies hebben laten zien dat tot $80 \%$ van de individuen die met een dieet gewicht verliezen, na 1 jaar weer significant in gewicht zijn gestegen. Gebleken is dat het vetweefsel een belangrijke rol speelt in het verhoogde risico op gewichtstoename na gewichtsverlies. Als men een dieet volgt ontstaat er een negatieve energiebalans omdat er minder energie wordt ingenomen dan het lichaam eigenlijk nodig heeft. Tijdens deze negatieve energiebalans verliezen vetcellen hun vet waardoor het volume van deze cellen afneemt. Deze afname in volume zorgt ervoor dat de cel en de omliggende extracellulaire matrix (ECM) niet meer bij elkaar passen en dit veroorzaakt stress in de vetcellen. Deze stress kan op meerdere manieren worden opgelost. Ten eerste kan de ECM worden aangepast zodat het weer past bij het lagere volume van de vetcel. Ten tweede kan er opnieuw vet worden opgeslagen in de cel zodat het volume toeneemt en de vetcel daardoor weer past bij de ECM. Echter, het opnieuw opslaan van vet leidt ertoe dat het lichaamsgewicht van de persoon weer toeneemt. Dit alles suggereert dat stress in vetcellen, die ontstaat tijdens een negatieve energiebalans doordat het volume van de cel niet meer past bij de omliggende ECM, een drijvende kracht achter het risico op gewichtstoename is. In de studies die beschreven staan in dit proefschrift onderzochten we de betrokkenheid van stress- en ECM-gerelateerde factoren binnen het vetweefsel in het proces van gewichtstoename na gewichtsverlies.

Ten eerste hebben we gekeken naar de relatie tussen de ECM-gerelateerde factoren en het risico op gewichtstoename in twee onafhankelijke studies.

In Hoofdstuk 2 hebben we gekeken naar de invloed van genetische variatie in 124 ECM-gerelateerde genen op het risico van gewichtstoename na afvallen onder de deelnemers van een pan-Europese, gerandomiseerde gewichtsverliesstudie (de zogenaamde "DiOGenes studie"). In deze studie werden personen met overgewicht en obesitas (310 vrouwen, 159 mannen) op een caloriearm dieet gehouden gedurende 8 weken en daarna werden deze personen nog gedurende 6 maanden (followup periode) gevolgd. Het lichaamsgewicht werd gemeten voor en na het dieet en na follow-up. Deze waarden zijn gebruikt om een score te berekenen die weergeeft hoeveel iemand weer is aangekomen in gewicht na het afvallen: (gewicht na follow-up - gewicht na afvallen) $\div$ (gewicht voor afvallen - gewicht na afvallen). Een score gelijk of lager dan nul geeft aan dat de persoon niet in gewicht is aangekomen of zelfs nog meer gewicht heeft verloren tijdens follow-up, terwijl een score hoger dan nul betekent dat het gewicht van de persoon is toegenomen tijdens follow-up. Daarnaast hebben we de gegevens van 2903 genetische variaties in en nabij de 124 ECM-gerelateerde genen verkregen. Met behulp van correlatie analyses is de relatie tussen de berekende score en de genetische variaties bepaald. Hierbij zagen we dat bij mannen variaties in de POSTN, LAMB1, COL23A1 en FBLN5 genen verband houden met het risico op gewichtstoename na gewichtsverlies. Bij vrouwen is een variant van het gen FN1 geassocieerd met het risico van gewichtstoename. 
Hoofdstuk 3 richt zich op de relatie tussen gewichtstoename en veranderingen in expressie van ECMgerelateerde genen. Dit werd onderzocht in een gerandomiseerde gewichtsverlies studie (de zogenaamde "jojo-studie") waarin 61 deelnemers gewicht verloren door een zeer laagcalorisch dieet (VLCD, 500 kilocalorieën/dag) te volgen voor 5 weken of een laagcalorisch dieet (LCD, 1250 kilocalorieën/dag) voor 12 weken. Daarna volgde er een gewichtsstabiele periode van 4 weken en een follow-up periode van 9 maanden. De gewichtsverlies periode (WL) en de gewichtsstabiele (WS) periode samen heten de dieet-interventie (DI) periode. Het gewichtstoename percentage (WR\%) werd berekend als waarde voor de gewichtstoename tijdens de follow-up: ((gewicht na follow-up gewicht na WS) $\div$ gewicht na WS) $\times 100 \%$.

Vervolgens is de gen expressie verandering van $277 \mathrm{ECM}$-gerelateerd genen tijdens WL, WS and DI gecorreleerd met de WR\%. We zagen dat 25 genen sterk correleerde met de WR\% en het hoogste aantal correlaties werd waargenomen tijdens de WS fase in de VLCD groep. Vijf van deze genen bleken te behoren tot een groep van 26 genen waarvan de expressieverandering sterk correleerde met elkaar. Deze groep genen kan worden onderverdeeld in 3 clusters, waarvan één voornamelijk bestaat uit genen die coderen voor leukocyt-specifieke integrines. Het lijkt zo te zijn dat een geringere vermindering van expressie van deze specifieke integrines tijdens de WS fase leidt tot een hoger risico op gewichtstoename en tevens lijkt dit samen te hangen met aanpassingen van de ECM. Het is mogelijk dat een sterkere vermindering van de capiciteit om de ECM aan te passen kan leiden tot het vasthouden van immuun cellen in het vetweefsel. Dit suggereert dat het risico op gewichtstoename wordt vergroot door behoud van ontstekingen van het vetweefsel kort na gewichtsverlies.

Ten tweede hebben we gekeken naar het verband tussen de stress-gerelateerde factoren en het risico op gewichtstoename in twee onafhankelijke studies.

In Hoofdstuk $\mathbf{4}$ hebben we onderzocht of de niveaus van specifieke stress eiwitten tijdens het gewichtsverlies en de follow-up gerelateerd zijn aan het risico voor gewichtstoename. Daartoe hebben 18 gezonde personen met overgewicht of obesitas een zeer-laagcalorisch dieet gevolgd voor 8 weken en 10 maanden daarna werden de personen opnieuw gezien. De personen werden vervolgens ingedeeld als zijnde 'gewichtsstabiel' of 'gewichtstoename', afhankelijk van de gewichts veranderingen tijdens de studie. Tot de gewichtsstabiele groep behoorden de personen die tenminste $10 \%$ van hun gewicht verloren hadden tijdens de dieetperiode en niet meer dan $6 \%$ waren aangekomen in gewicht tijdens de follow-up. Tot de gewichtstoename groep behoorden de personen die tenminste $10 \%$ van hun gewicht verloren hadden maar meer dan $6 \%$ aankwamen in gewicht tijdens de follow-up. Na het afvallen werden de niveaus van de verschillende stress eiwitten in het vetweefsel vergeleken tussen de 2 groepen en daarbij kwam aan het licht dat de niveaus van $\beta$-actin, calnexin, heat shock protein (HSP) 27, HSP60 en HSP70 hoger waren in de gewichtstoename groep. De veranderingen tijdens gewichtsverlies van $\beta$-actin, HSP27 en HSP70 bleken gerelateerd aan veranderingen van HSP60, een mogelijk belangrijke factor in het risico voor gewichtstoename na het afvallen. Deze bevindingen bevestigen dat stress in vetcellen een rol speelt in het risico op gewichtstoename.

Hoofdstuk 5 geeft informatie over de relatie tussen gewichtstoename en veranderingen in expressie van stress-gerelateerde genen tijdens de WL, WS en DI in de jojo-studie. Expressie veranderingen van 107 stress-gerelateerde genen tijdens WL, WS en DI werden gecorreleerd met het WR\%. We zagen dat er meer correlaties tussen de WR\% en gen expressie veranderingen waren in de VLCD groep ten opzichte van de LCD groep. Deze correlaties waren vooral te zien tijdens de WS en DI periode. Acht 
van de negen gecorreleerde stress genen tijdens de WS fase waren aan elkaar gelinkt. Deze genen geven aan dat er een verband bestaat tussen gewichtstoename en bepaalde biologische processen in het vetweefsel: actine filament dynamica, glucose metabolisme en nutrient sensing.

Ten derde hebben we in de jojo-studie gekeken naar de interactie tussen de stress- en ECMgerelateerde factoren en hun invloed op het risico van gewichtstoename.

In Hoofdstuk 6 werden correlatie en interactie analyses uitgevoerd met stress- en ECM-gerelateerde genen om te achterhalen of combinaties van genen een effect hebben op het WR\%. Uit de correlatie analyses bleek dat er tijdens de WS fase in de VLCD groep een netwerk kan worden gevormd bestaande uit 8 stress- en 15 ECM-gerelateerd genen, die allemaal correleren met de WR\%. Het netwerk linkt aan specifieke biologische processen in het vetweefsel: leukocyten-activiteit, ECM aanpassingen, actine cytoskelet organisatie en glucose metabolisme. Interactie analyses lieten zien dat verschillende combinaties van stress en ECM genen een significant effect hebben op het WR\%. Vooral de signalering door de epidermale groeifactor werd geïdentificeerd als sterke beïnvloeder van het risico op gewichtstoename waarbij actinine alfa-1 (ACTN1), integrine beta 4 (ITGB4), cystatine C ( CST3) en laminine alfa-3 (LAMA3) een rol spelen.

Dit proefschrift biedt belangrijke inzichten in het onderliggende biologische mechanisme van gewichtstoename na gewichtsverlies. We hebben aangetoond dat het risico van hernieuwde gewichtstoename samenhangt met celstress in het vetweefsel. Tekenend daarvoor zijn de expressie veranderingen van verschillende stress- en ECM-gerelateerde genen gedurende de eerste vier weken van terugkeer in energiebalans. Daarnaast laten we zien dat de interacties tussen de stress- en ECMgerelateerde genen een effect hebben op de gewichtstoename na het afvallen. Tevens hebben we aangetoond dat gewichtstoename geassocieerd is met genetische variaties van ECM genen, die verschillend zijn voor vrouwen en mannen. 


\section{Valorisation}


This thesis focusses on extending the fundamental knowledge about the underlying process of the seemingly inevitable weight regain after weight loss of overweight and obese humans.

\section{Social and economic relevance}

The prevalence of obesity has reached epidemic proportions. In 2014, the World Health Organization estimated that $39 \%$ of adults aged 18 years and over were overweight (BMI $\geq 25)$ and $13 \%$ were obese (BMI>30) (1). This means that more than 1.9 billion adults are affected by overweight including obesity. Worldwide, overweight tends to be more common for woman compared to men $(40 \%$ women vs $38 \%$ men), which is similar for obesity ( $15 \%$ women vs $11 \%$ men). The global prevalence of obesity has more than doubled between 1980 and 2014. The increasing prevalence of obesity is a major health concern since it increases the risk for developing type 2 diabetes mellitus, cardiovascular diseases and several types of cancer (2-4). In 2010, around 3.4 million adults died as a result of being overweight or obese (5). In addition to the health problems, overweight and obesity have a considerable economic impact and increase healthcare costs. In the Netherlands, the total direct and indirect costs of overweight and obesity is estimated at 3 billion euros per year. Of these 3 billion euros, 1.2 billion is directly linked to healthcare costs caused by overweight and obesity and 2 billion are linked to the lost productivity which is a result from days taken off sick. These facts clearly indicate that obesity and its associated diseases have a profound impact on our society.

The remedy to obesity is losing weight which can be achieved by limiting energy/food intake and increasing daily physical activity for a longer period of time. A reduction in body weight lowers the risk for developing diseases and improves the metabolic profile in overweight and obese people (68). However, long-term weight loss maintenance has been proven to be difficult $(9,10)$. Generally, up to $80 \%$ of the people are unsuccessful in maintaining weight loss $(11,12)$. It is crucial to prevent or reduce weight regain on a long-term basis since it would lead to a tremendous reduction of the disease burden and the financial costs related to the diseases. This thesis provides more insight into the mechanisms involved in the biological process for weight regain which can contribute to solving the problem of the seemingly inevitable weight regain after weight loss.

\section{Scientific gain of this thesis}

During a negative energy balance, cellular stress may arise in adipocytes due to the misfit between the lowered cell volume and the surrounding ECM. This stress needs to be resolved which can be achieved by remodeling of the ECM or by the re-storing of triglycerides within the adipocytes to increase the cell volume. This thesis focused on determining the involvement of stress- and ECMrelated factors in the underlying mechanisms of weight regain.

- We have found that not the weight loss period per se is crucial in the underlying mechanisms involved in weight regain, but that especially the weeks after weight loss, when returning to energy balance, are linked to the risk of weight regain. In other words, studying the period after weight loss, when people increase their food intake to meet their energy requirements, may lead to the identification of risk markers for weight regain after weight loss.

- We have shown that changes of stress- and ECM-related genes and the interaction of these changes during the weeks after weight loss, when returning to energy balance, are highly related with weight regain during follow-up. Studying these stress and ECM genes, which are linked to several biological processes, may lead to the identification of genetic risk factors 
involved in the process of weight regain after weight loss and may provide targets for intervention.

- We have observed that people have a higher risk for weight regain when there is a stronger downregulation of ECM-remodelling when returning to energy balance. This may lead to the retaining of more immune cells in the adipose tissue suggesting that resident inflammation after weight loss increases the risk for weight regain. Studying inflammation after weight loss may lead to the identification of risk markers for weight regain and inflammation-related targets for intervention.

- Several of the investigated single nucleotide polymorphisms (SNPs) of ECM genes are linked with weight regain. Possibly, these SNPs can be used to create a genetic risk profile to select people that are at high risk of weight regain, who can be better guided to achieve weight maintenance after weight loss.

\section{Innovation}

The present thesis presents various novel findings and insights. The aim was to determining the involvement of stress- and ECM-related factors in the underlying mechanisms of weight regain. We were able to show that the change of multiple stress-related markers influenced the regain of weight during follow-up, which confirms the proposed role of cellular stress as a driver of weight regain. Surprisingly, the change during the weeks after weight loss seems at least as important in this process as the change during weight loss. Similar to the stress markers, we showed that changes of ECM-related genes after weight loss were associated with weight regain. Here, we specifically saw that when returning to energy balance a stronger reduction of ECM remodelling capacity leads to more retention of immune cells in the adipose tissue. This indicates that inflammation after weight loss increases the risk for weight regain and as such adds a novel dimension to the biological mechanism of weight regain. However, stress- and ECM-related factors are not only influencing the risk for weight regain separately but also have a combined effect. Several stress and ECM gene interactions were highly related to weight regain after weight loss. Together these interactions indicate that changes in the epidermal growth factor signalling, when returning to energy balance, influence the risk of weight regain, which may be mediated by alpha-actinin phosphorylation and modified by expression of integrin beta 4 , cystatin $C$ and laminin alpha-3. It connects endocrine or autocrine signals, yet to be identified, to adipocyte stress as a factor for weight regain.

Furthermore, in this thesis we have shown that several SNPS of ECM genes are linked with weight regain. In men, SNPs of the ECM genes collagen type XXIII alpha1, fibulin-5, laminin- $\beta 1$ and, periostin were linked while in women fibronectin 1 was linked. These SNPs could add to a genetic risk profile for weight regain. The SNPs were different for men and woman, which is not uncommon for research on weight regulation, underscoring the importance for sex-specific research.

\section{Target groups}

The results described in this thesis are available for the scientific community through publications in international peer-reviewed journals. Also, results were presented at important international congresses. Yet, the most important target group is society itself. News articles on our research have been published on various news websites, which has enabled a broader audience to take notice of our results. The scientific gain of this thesis will help to fight obesity on the long-term by determining the risk for weight regain of overweight and obese subjects. People with a higher risk can be guided 
better by providing a specific, personalized guideline during weight loss but especially also during weight maintenance. This will enhance the efficiency of the weight loss treatment and of weight maintenance, which will contribute to the reduction of the health burden for society. For example, people with an increased risk for weight regain should receive a more stringent and/or frequent guidance than those with a lower risk. To achieve this, high quality prediction models are needed, which requires collaborations between research groups and companies to explore large-scale genome data. The prediction models and treatment guidelines can be used by trained dieticians, specialists in the hospital and obesity clinics.

\section{Planning and implementation}

The prediction models and treatment guidelines need to be made, however, this requires collaborations with other research groups within and outside the university as well as collaborations with the industry. For example, bioinformatics and biostatistics with a focus on modelling are needed to use the available data and create a clear model for weight regain. These collaborations will lead to better and more accurate insight into the underlying mechanism for the risk weight regain and the risk condition of each individual. The findings presented in this thesis are the starting point in this respect and at the moment we are collaborating with the Maastricht Centre for Systems Biology (MaCSBio) to create a model for weight regain. Within the coming years this model will be finished and available for society. This model will lead to better and more accurate insight into the process of weight regain and assist the specialists to provide specific, personalized guidance to people to create optimal conditions for weight maintenance after weight loss. It should be noted that this model requires the use of genetic information of individuals and this might lead to ethical issues, especially about privacy of the individual. Privacy concerns arise because organisations and institutions might be interested in knowing a person's genetic status which may result in stigmatization, discrimination, and other adverse effects. For example, if an insurance company knows that an individual is predisposed to develop obesity by looking at his/hers genetic information, they might increase the individual's insurance bill already to prevent possible extra costs later on, while the individual might never get sick. On the other hand, it might be beneficial to know if someone is predisposed to develop a specific disease because than actions can be taken to prevent the disease from occurring. Those and other questions have still to be worked out for a complex trait as overweight and obesity which requires the active involvement of scientists from multiple disciplines and of politicians. 


\section{References}

1. WHO. Obesity and overweight. Fact sheet №311, 2016.

2. Kahn SE, Hull RL, Utzschneider KM. Mechanisms linking obesity to insulin resistance and type 2 diabetes. Nature, 2006. 444(7121):840-6.

3. Van Gaal LF, Mertens IL, De Block CE. Mechanisms linking obesity with cardiovascular disease. Nature, 2006. 444(7121):875-80.

4. Renehan AG, Zwahlen M, Egger M. Adiposity and cancer risk: new mechanistic insights from epidemiology. Nature reviews Cancer, 2015. 15(8):484-98.

5. Lim SS, Vos T, Flaxman AD, Danaei G, Shibuya K, Adair-Rohani H et al. A comparative risk assessment of burden of disease and injury attributable to 67 risk factors and risk factor clusters in 21 regions, 1990-2010: a systematic analysis for the Global Burden of Disease Study 2010. Lancet, 2012. 380(9859):2224-60.

6. Horton ES. Effects of lifestyle changes to reduce risks of diabetes and associated cardiovascular risks: results from large scale efficacy trials. Obesity (Silver Spring), 2009. 17 Suppl 3:S43-8.

7. King RJ, Ajjan RA. Vascular risk in obesity: Facts, misconceptions and the unknown. Diab Vasc Dis Res, 2017. 14(1):2-13.

8. Weinstock RS, Dai H, Wadden TA. Diet and exercise in the treatment of obesity: effects of 3 interventions on insulin resistance. Arch Intern Med, 1998. 158(22):2477-83.

9. Weiss EC, Galuska DA, Kettel Khan L, Gillespie C, Serdula MK. Weight regain in U.S. adults who experienced substantial weight loss, 1999-2002. American journal of preventive medicine, 2007. 33(1):34-40.

10. Sarlio-Lahteenkorva S, Rissanen A, Kaprio J. A descriptive study of weight loss maintenance: 6 and 15 year follow-up of initially overweight adults. Int J Obes Relat Metab Disord, 2000. 24(1):116-25.

11. Barte JC, ter Bogt NC, Bogers RP, Teixeira PJ, Blissmer B, Mori TA, Bemelmans WJ. Maintenance of weight loss after lifestyle interventions for overweight and obesity, a systematic review. Obes Rev, 2010. 11(12):899-906.

12. Wu T, Gao X, Chen M, van Dam RM. Long-term effectiveness of diet-plus-exercise interventions vs. diet-only interventions for weight loss: a meta-analysis. Obes Rev, 2009. 10(3):313-23. 


\section{Dankwoord/Acknowledgements}


Eindelijk is mijn proefschrift af! Ik heb er de afgelopen jaren met veel plezier aan gewerkt maar het was uiteraard nooit alleen gelukt, daarom wil ik graag iedereen bedanken die op welke manier dan ook heeft bijgedragen aan dit proefschrift.

Allereerst wil ik mijn promotoren Prof. dr. Edwin Mariman en Prof. dr. Marleen van Baak graag bedanken voor de steun en begeleiding die ik de afgelopen jaren van hen heb gekregen. Edwin, bedankt dat je tijdens mijn promotie altijd voor mij klaar stond. Met rust en geduld heb je mij altijd geholpen om mijn eigen weg te vinden. Dank voor de mooie en leerzame tijd. Marleen, bedankt voor de fijne samenwerking. Ik kon altijd bij je terecht met mijn vragen en ik heb veel waardevolle tips van jou gekregen voor het verbeteren van experimenten en manuscripten. Ik heb ontzettend veel van je geleerd en wil je daar graag voor bedanken.

Verder zou ik graag de beoordelingscommissie bestaande uit Prof. dr. Ellen Blaak, Prof. dr. Ilja Arts, Prof. dr. Sander Kersten, Dr. Evert van Schothorst en Prof. dr. Patrick Schrauwen bedanken voor de tijd en moeite die jullie hebben genomen om mijn proefschrift te beoordelen.

Roel, samen hebben we hard gewerkt om van het jojo-project een succes te maken en het is ons goed gelukt. Ik kon altijd bij jou terecht als ik een vraag of probleem wat heeft geleid tot menige discussie over de beste aanpak. Jouw kennis, vrolijke karakter en harde werken hebben veel betekend voor het jojo-project en voor mijn proefschrift waarvoor ik je ontzettend dankbaar ben!

Laura, naast al je goede werk met de proefpersonen stond je ook nog eens altijd klaar voor Roel en mij als we ergens anders hulp bij nodig hadden. Naast alle werkzaamheden heb ik je ook leren kennen op persoonlijk vlak. Wij konden het meteen goed met elkaar vinden. Ik denk met plezier terug aan onze koffiepauzes en lunches waarbij we naast veel gezelligheid ook een luisterend oor hadden voor elkaars problemen. Bedankt voor de leuke tijd!

Vervolgens wil ik graag mijn collega's binnen de FunGen groep bedanken. Johan, ik bewonder je om je werklust en je enthousiasme. Ik kon altijd bij je terecht als ik advies nodig had voor mijn project maar daarnaast hebben we menig uurtje gevuld met persoonlijke gesprekken over vanalles en nog wat. Freek, zowel op het lab als daarbuiten heb ik veel van je geleerd. Bij jou kon ik altijd langskomen met vragen of problemen en je stond meteen klaar om te helpen. Ping, many times I mailed you to ask for help and without any doubt you would always help me as soon as you could. You always made time to read, discuss and improve my work, thank you! Qi, it was nice to have you as a roommate, we had so much fun together. Thank you for being such a good friend and good luck with your career. Daniela, you joined our group for a short period but during that time I got to know you as a fun person with whom I could share my personal life. I wish you and your family all the best and good luck with your future research in Romania.

Uiteraard wil ik ook graag mijn twee paranimfen bedanken. Birgitta, ten eerste wil ik je bedanken voor het altijd meedenken en meediscussiëren over de aanpak van mijn project. Je hebt me met regelmaat nuttig advies gegeven waardoor ik weer verder kon met mijn project. Daarnaast zorgde je er voor dat er ook tijd werd gemaakt voor de nodige afleiding van het werk. Het was altijd fijn om even een gezellig praatje met jou te hebben. Bedankt voor alle hulp, advies en leuke gesprekken en ik ben blij dat je aan mijn zijde wilt staan tijdens mijn verdediging. Qing, you were always interested 
in knowing how my work was going and whenever I was stuck you always tried to help me. But besides our work I also got to know you personally. You are a nice and fun person and we had many conversations with each other about the most divers themes (I definitely learned a lot about China and its culture). Thank you for always being there for me. Good luck with finishing you PhD but besides that enjoy your time with your husband and your little bundle of joy.

Also, I would like to thank Mattea, Birgitta, Qing, Qi, Dorien, Jessica, Elaine, Rudi, Max, Adriyan, Rens, Emanuel, Manuel for the fun during and outside of work. I enjoyed our regular lunches at work but also had a lot of fun with our activities outside of work such as the Hot Pot, table tennis, Glow. These evening were always filled with lots of joy and laughter.

Natuurlijk wil ik ook alle andere collega's van de afdeling Humane Biologie bedanken voor de prettige werksfeer. Ik heb met heel veel plezier op deze afdeling gewerkt en dit kwam mede door alle fijne collega's. Er was een fijne, relaxte sfeer op de werkvloer en naast de uren op de afdeling waren er de leuke borrels, feestjes, HB-uitjes, kerstdiners etc. die voor extra gezelligheid hebben gezorgd. Bedankt!

Verder wil ik graag mijn beste vriendinnen bedanken. Astrid, Cara en Peggy, dankjulliewel dat ik bij jullie altijd terecht kan voor het delen van mijn lief en leed. Daarnaast geniet ik van onze activiteiten zoals het escapen, uiteten, borrelen en de spelletjes waarbij we regelmatig met $z^{\prime} n$ allen in een deuk liggen van het lachen. Ik ben blij dat we na al deze jaren nog vriendinnen zijn en dit zal in de toekomst zeker zo blijven. Bedankt!

Dan wil ik ook mijn familie bedanken. Regelmatig hebben jullie gevraagd naar mijn precieze werkzaamheden waarna jullie met geduld en interesse geluisterd hebben naar mijn poging om dit uit te leggen. Het leidde er zelfs toe dat ik Nederlandse samenvattingen met illustraties maakte van mijn gepubliceerde artikelen zodat het beter te begrijpen was. Ik weet niet of het altijd veel duidelijker werd maar ik vond het fijn dat jullie altijd interesse hebben getoond in mijn werk en ik wil jullie hiervoor bedanken.

Kim en Annelies, bedankt voor alle steun. Het is een fijn gevoel om te weten dat ik altijd op jullie kan rekenen als dat nodig is. Ik wens jullie veel plezier in jullie nieuwe, eigen huis. Daarnaast kijk ik met veel plezier en trots uit naar de geboorte van jullie eerste spruit en dat ik mijzelf officieël tante mag gaan noemen :)

Mam en Pap, ik kan jullie niet genoeg bedanken voor alles wat jullie voor mij gedaan hebben. Jullie hebben mij altijd gemotiveerd om het maximale uit mijzelf te halen en mede daardoor is mijn promotie een succes geworden. Daarnaast kan ik altijd bij jullie binnen lopen als ik mijn hart wil luchten of om een lekkere maaltijd te eten. Ook zijn jullie altijd bereid om op mijn monster Odie te passen waarbij ik weet dat hij zeker verwend wordt. Bedankt dat jullie altijd voor mij klaar staan. Ik hou van jullie! 


\section{Curriculum Vitae}


Nadia Roumans was born on March $3^{\text {rd }} 1989$ in Zeven, Germany, and raised in Eygelshoven, the Netherlands. She completed secondary school at College Rolduc in Kerkrade in 2006. Consecutively, she studied Biology and Medical Laboratory Research at Hogeschool Zuyd in Heerlen, for which she received her Bachelor's degree in 2010. She continued with a 2-year master in Molecular Life Sciences, with a specialization in Clinical Molecular Sciences, at Maastricht University, for which she received her Master's degree in 2012. In November 2012, Nadia started working as a PhD-candidate at the department of Human Biology of the Faculty of Health, Medicine and Life Sciences of Maastricht University under the supervision of Prof. dr. Edwin Mariman and Prof. dr. Marleen van Baak. During this period, she investigated the involvement of stress- and extracellular matrix-related factors in the risk of weight regain after weight loss, as demonstrated in this thesis and publications in scientific journals. 


\section{List of Publications}


Tareen SHK, Adriaens ME, Arts ICW, de Kok T, Vink RG, Roumans NJT, van Baak MA, Marimen ECM, Evelo CT, Kutmon M. Profiling core processes in adipose tissue during weight loss using time series gene expression. Submitted for publication.

Roumans NJ, Vink RG, Wang P, van Baak MA, Mariman EC. Relation between stress- and ECM-related genes and their effect on weight regain. To be submitted.

Roumans NJ, Vink RG, Fazelzadeh P, van Baak MA, Mariman EC. A role for leukocyte and ECM remodelling of adipose tissue in the risk of weight regain after weight loss. American Journal of Clinical Nutrition. 2017; 105(5):1054-1062.

Vink RG, Roumans NJ, Čajlaković M, Cleutjens JPM, Boekschoten MV, Fazelzadeh P, Vogel MAA, Blaak EE, Mariman EC, van Baak MA, Goossens GH. Diet-induced weight loss decreases adipose tissue oxygen tension with parallel changes in adipose tissue phenotype and insulin sensitivity in overweight humans. International Journal of Obesity. 2017; 41(5):722-728.

Vink RG, Roumans NJ, Mariman EC, van Baak MA. Dietary weight loss-induced changes in RBP4, FFA and ACE predict weight regain in people with overweight and obesity. Submitted for publication.

Vink RG, Roumans NJ, van der Kolk BW, Fazelzadeh P, Boekschoten MV, Mariman EC, van Baak MA. Adipose tissue meal-derived fatty acid uptake is not enhanced after diet-induced weight loss in overweight and obese adults. Obesity. 2017; in press.

Roumans NJ, Vink RG, Bouwman FG, Fazelzadeh P, van Baak MA, Mariman EC. Weight loss-induced cellular stress in subcutaneous adipose tissue and the risk for weight regain in overweight and obese adults. International Journal of Obesity. 2016; in press.

Vink RG*, Roumans NJ*, Fazelzadeh P, Tareen SH, Boekschoten MV, van Baak MA, Mariman EC. Adipose tissue gene expression is differentially regulated with different rates of weight loss in overweight and obese humans. International Journal of Obesity. 2017; 41(2):309-316.

* Both authors contributed equally.

Lenz M, Roumans NJT, Vink RG, van Baak MA, Mariman EC, Arts IC, de Kok TM, Ertaylan G. Estimating real cell size distribution from cross-section microscopy imaging. Bioinformatics. 2016; 32(17): i396i404.

Mariman EC, Vink RG, Roumans NJ, Bouwman FG, Stumpel CT, Aller EE, van Baak MA, Wang P. The cilium: a cellular antenna with an influence on obesity risk. British Journal of Nutrition. 2016; 116(4): 576-592

Roumans NJ, Camps SG, Renes J, Bouwman FG, Westerterp KR, Mariman EC. Weight loss-induced stress in subcutaneous adipose tissue is related to weight regain. British Journal of Nutrition. 2016; 115(5): 913-920

Vink RG, Roumans NJ, Arkenbosch LA, Mariman EC, van Baak MA. The effect of rate of weight loss on long-term weight regain in adults with overweight and obesity. Obesity. 2016; 24(2): 321-327.

Roumans NJ, Vink RG, Gielen M, Zeegers MP, Holst C, Wang P, Astrup A, Saris WH, Valsesia A, Hager $J$, van Baak MA, Mariman EC. Variation in extracellular matrix genes is associated with weight regain after weight loss in a sex-specific manner. Genes \& Nutrition. 2015; 10(6): 56.

Camps SG, Verhoef SP, Roumans N, Bouwman FG, Mariman EC, Westerterp KR. Weight loss-induced changes in adipose tissue proteins associated with fatty acid and glucose metabolism correlate with adaptations in energy expenditure. Nutrition \& metabolism. 2015; 12: 37. 
Renes J, Rosenow A, Roumans NJ, Noben JP, Mariman EC. Calorie restriction-induced changes in the secretome of human adipocytes, comparison with resveratrol-induced secretome effects. Biochimica et biophysica acta. 2014; 1844(9): 1511-1522.

Jetten N, Roumans N, Gijbels MJ, Romano A, Post MJ, de Winther MP, van der Hulst RR, Xanthoulea $\mathrm{S}$. Wound administration of M2-polarized macrophages does not improve murine cutaneous healing responses. PlosOne. 2014; 9(7): e102994. 